\title{
A Formalised Approach to the Management of Risk
}

by

Michael J Brownsword, MEng(Hons)

Thesis submitted for the Degree of Doctor of Philosophy

at

Cardiff University

April 2009 
UMI Number: U585234

All rights reserved

\section{INFORMATION TO ALL USERS}

The quality of this reproduction is dependent upon the quality of the copy submitted.

In the unlikely event that the author did not send a complete manuscript and there are missing pages, these will be noted. Also, if material had to be removed, a note will indicate the deletion.

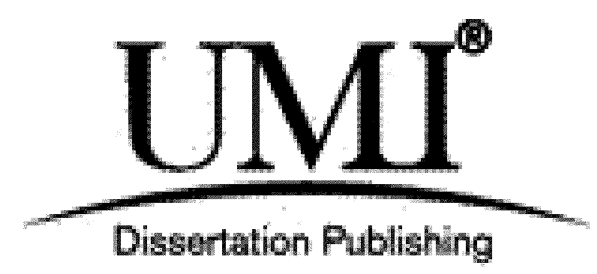

UMI U585234

Published by ProQuest LLC 2013. Copyright in the Dissertation held by the Author.

Microform Edition (c) ProQuest LLC.

All rights reserved. This work is protected against unauthorized copying under Title 17, United States Code.

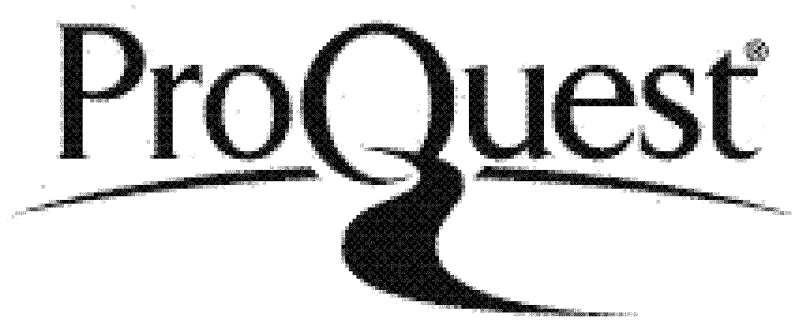

ProQuest LLC

789 East Eisenhower Parkway

P.O. Box 1346

Ann Arbor, MI 48106-1346 


\section{Declaration}

This work has not previously been accepted in substance for any degree and is not concurrently submitted in candidature for any degree.

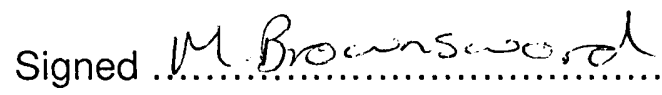
(candidate)

Date $2 c_{1} / \mathrm{Lr}_{\mathrm{H}} / \mathrm{c}$

\section{STATEMENT 1}

This thesis is being submitted in partial fulfillment of the requirements for the degree of $\mathrm{PhD}$

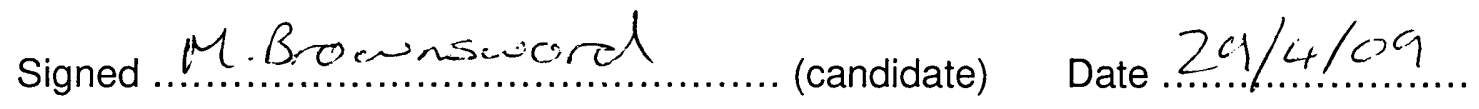

\section{STATEMENT 2}

This thesis is the result of my own independent work/investigation, except where otherwise stated.

Other sources are acknowledged by explicit references.

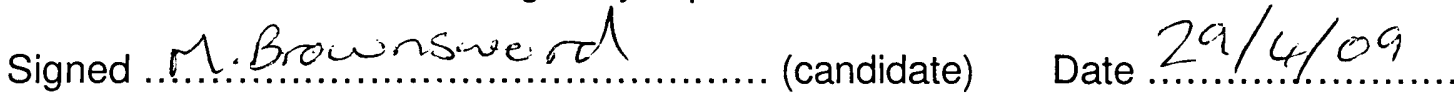

\section{STATEMENT 3}

I hereby give consent for my thesis, if accepted, to be available for photocopying and for inter-library loan, and for the title and summary to be made available to outside organisations.

Signed ...M. Browensceorol (candidate) 


\section{Acknowledgements}

I would like to thank Brass Bullet Ltd. whose funding made this project possible. I would specifically like to thank $\mathrm{Dr}$ Jon Holt for providing unwavering support, insight and clarity throughout the project.

My thanks go to Prof Mike Rodd and Dr Rossi Setchi as academic supervisors, their guidance, insight and drive has been invaluable.

Thanks also go to the Kings Norton Parish who have provided the backdrop for the main case study. Their willingness to try, acceptance of and openness to the results of a different way of working has been refreshing.

Thanks also go to the many friends and colleges who have indulged in conversation and debate regarding my thoughts and ideas, especially Dave and Simon who in any discussion will provide the most positive of criticism. This includes those I have worked with in many different establishments over the course of this work.

Almost finally my thanks go to Lesley, Peter, Bob and Mita who have all always been supportive and provided much needed food, drink, accommodation and child care.

And finally, to Jean, my dear wife, who has prodded and cajoled me into action; whilst reviewing various aspects of this work since we were married. She has provided me with two wonderful boys, Samuel and Daniel, who have provided much motivation over the last two years. 


\section{Abstract}

Taking a pragmatic, systems engineering approach, this thesis identifies a number of fundamental issues that presently arise in risk management, primarily as a result of the overly complex and somewhat outdated approach conventionally taken in process definition and a lack of coherence within the current risk management vocabulary. It is suggested that many recent developments in systems engineering have largely been ignored by the risk management community.

The objective of this work is to develop a formalised approach to the management of risk using a model based approach this will enable a fundamental simplification of the risk management process, resulting amongst other things - in an improved understanding of the associated terminology.

An object oriented modelling approach, now widely exploited in systems engineering, has been used to provide an insight into many existing risk management standards considering the approaches they present and terminology used. It has also been used to derive both a set of processes for risk management and a methodology for implementation. Alongside this, a consistent, inter-related terminology as been proposed for use with these processes.

The outcome of this work is a formalised but pragmatic approach to risk management including the definition of processes, ontology for risk management and a pragmatic methodology for the application of the processes. This approach has been validated in a number of case studies of varying depth and breadth, covering health \& safety, business, project and individual needs, showing that the proposed processes and terminology can be used effectively in different organisations and industries. 


\section{Table of Contents}

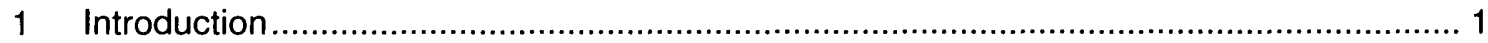

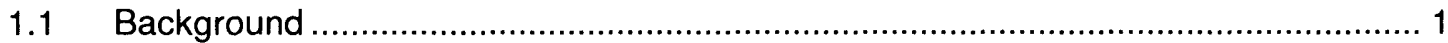

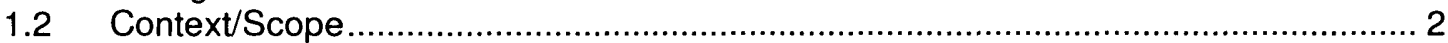

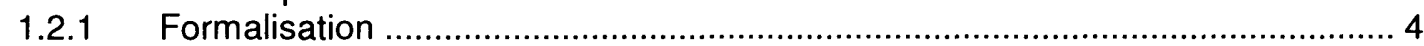

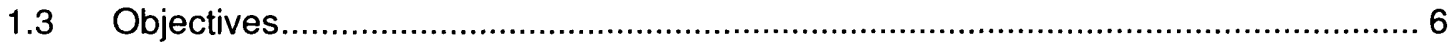

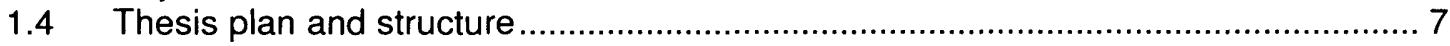

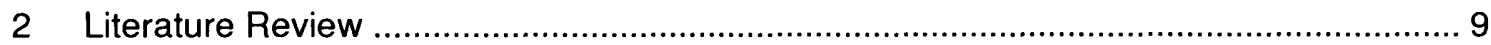

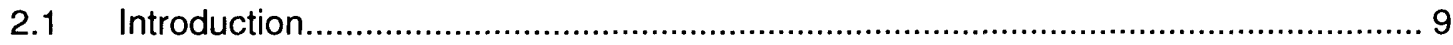

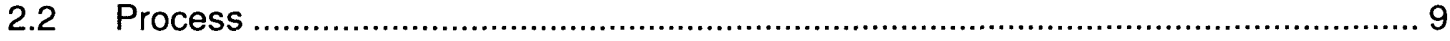

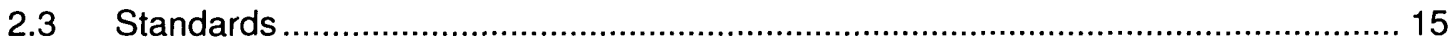

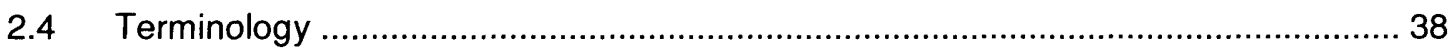

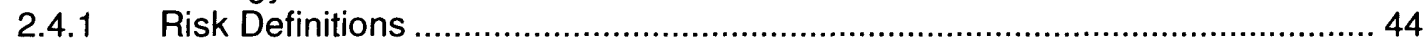

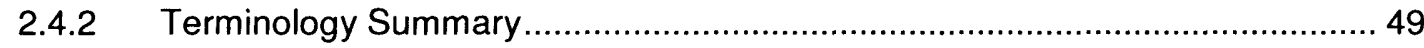

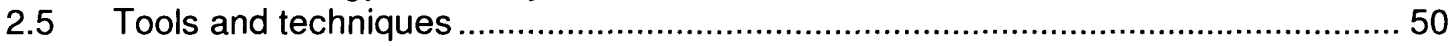

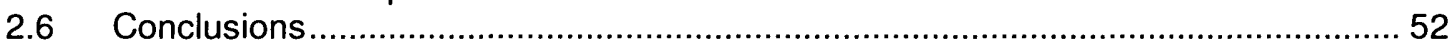

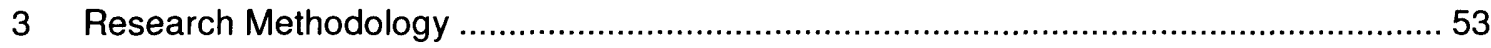

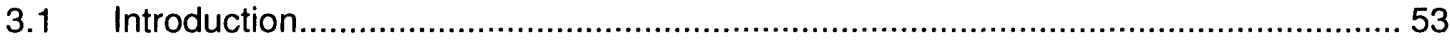

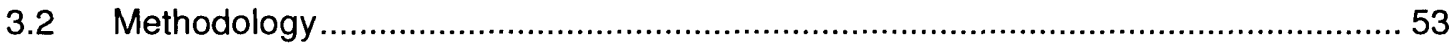

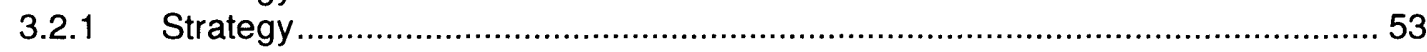

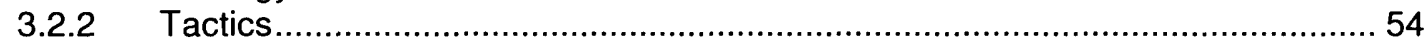

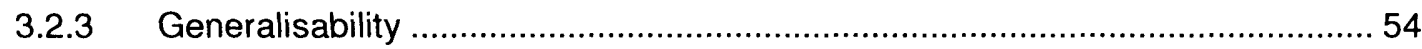

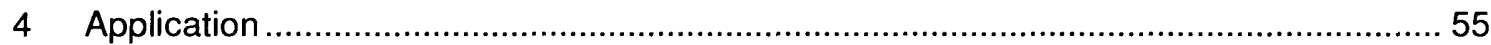

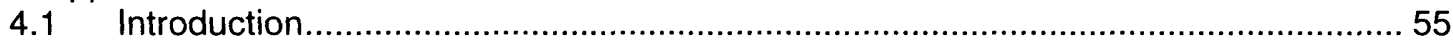

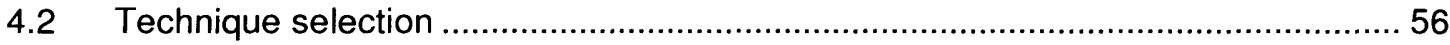

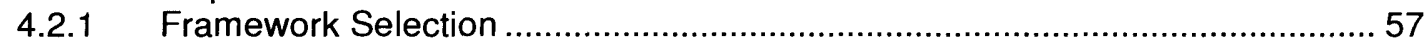

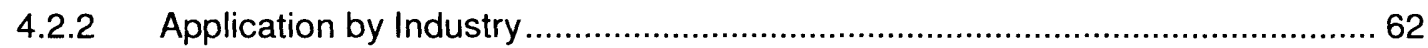

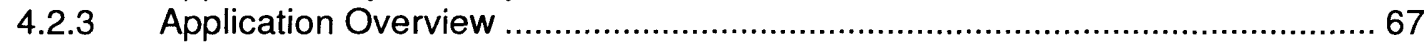

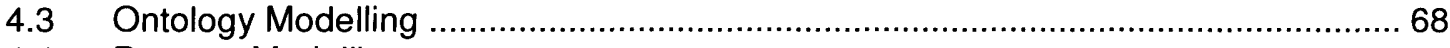

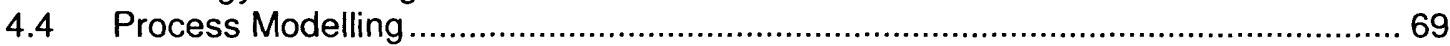

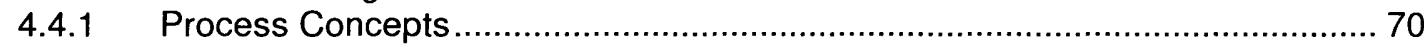

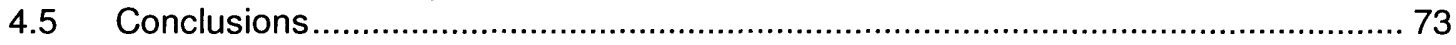

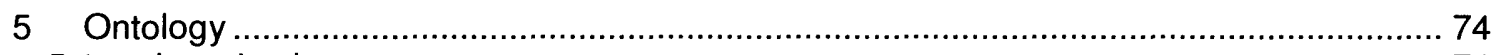

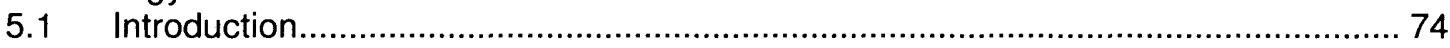

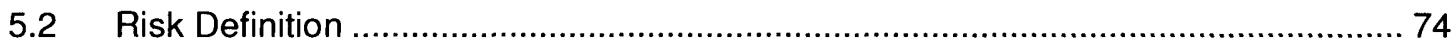

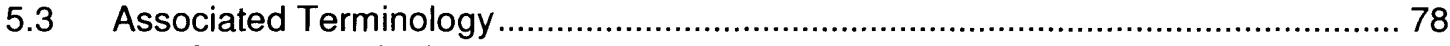

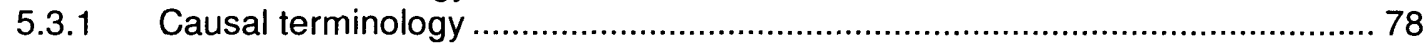

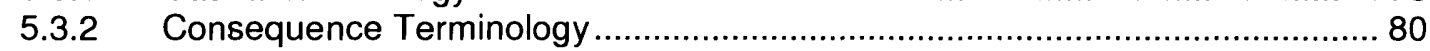

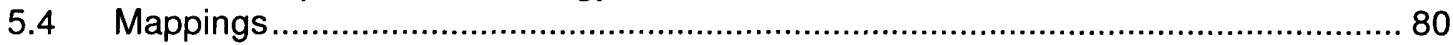

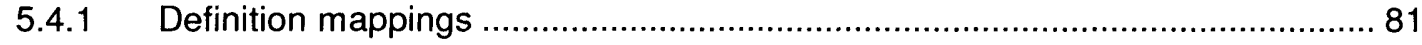

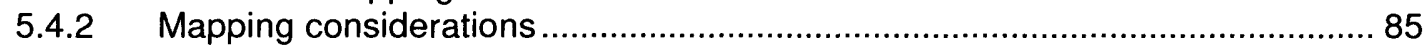

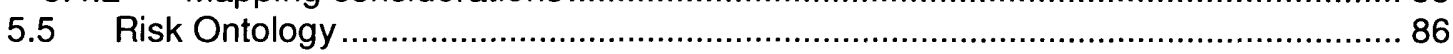

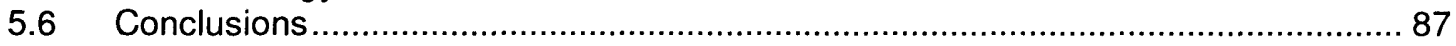




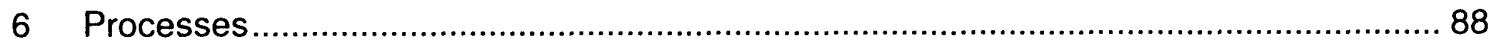

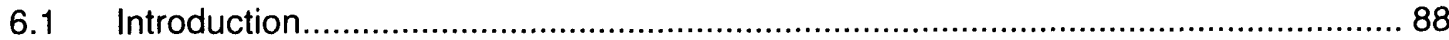

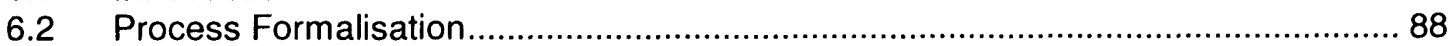

6.3 Requirements View - Risk Management Requirements ...................................... 89

6.4 Process Content View - Risk Processes...................................................... 90

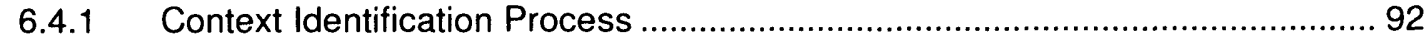

6.4.2 System Modelling Process .............................................................. 93

6.4.3 Concern Identification Process ........................................................... 94

6.4.4 Risk Definition Process .................................................................... 95

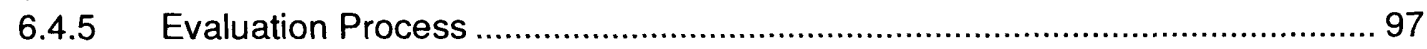

6.4.6 Risk Treatment Process.................................................................... 99

6.5 Information View - Risk Processes Artefacts ................................................ 101

6.6 Process Instance View - Methodology ...................................................... 102

6.7 Process Support................................................................................. 104

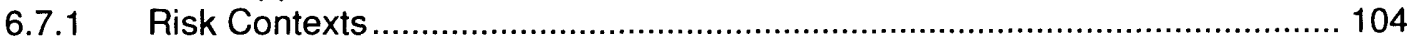

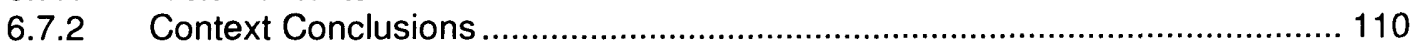

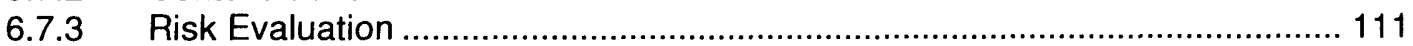

6.8 Conclusion..................................................................................... 113

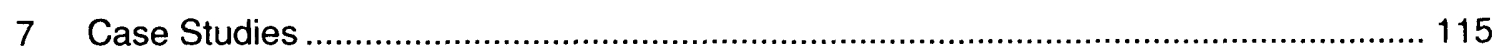

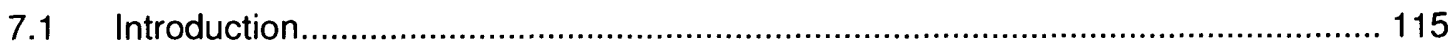

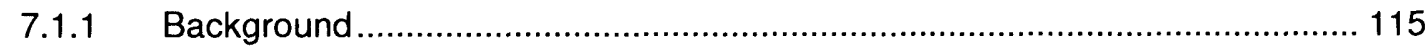

7.1.2 Case study tactics ..................................................................... 116

7.2 Approach .............................................................................................. 117

7.2.1 Initial Concern Identification................................................................. 118

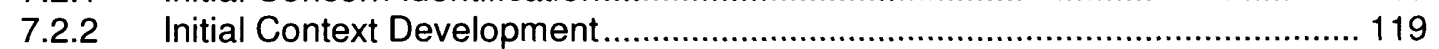

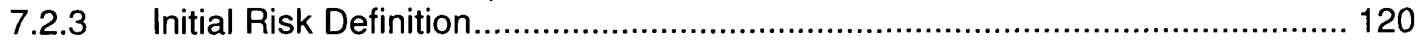

7.3 Health and Safety Risk..................................................................... 121

7.3.1 Understanding the System ............................................................. 122

7.3.2 Scalded child situation evaluation..................................................... 125

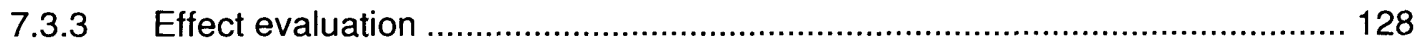

7.3.4 Treating the risk of scalding a child ................................................... 129

7.3.5 Additional Concerns........................................................................ 132

7.3.6 Scalded child Solution evaluation ..................................................... 133

7.3.7 Health and Safety Risk Conclusion ................................................. 133

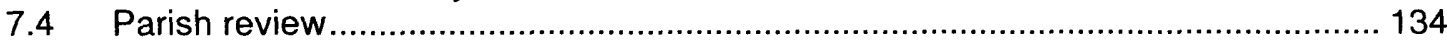

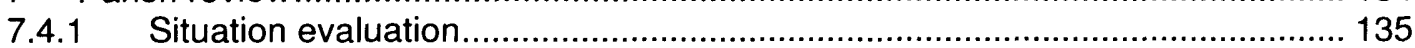

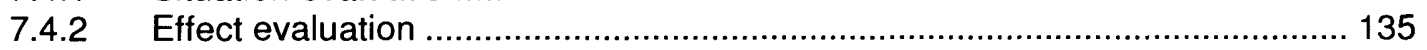

7.4.3 Treating the risk of not getting new clergy members .............................. 136

7.5 Conclusions ....................................................................................... 138

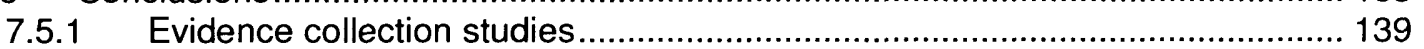

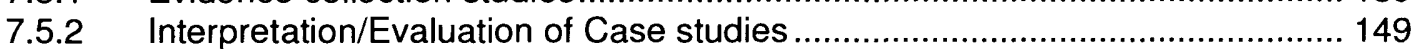

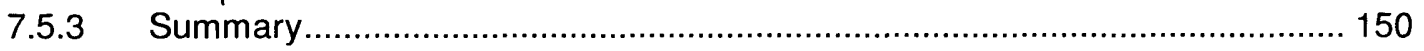

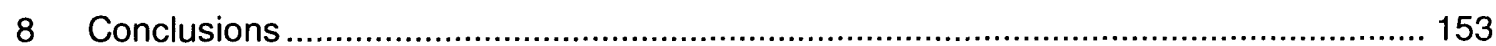

8.1 Introduction .................................................................................... 153

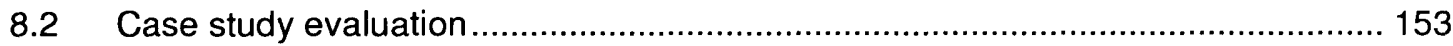

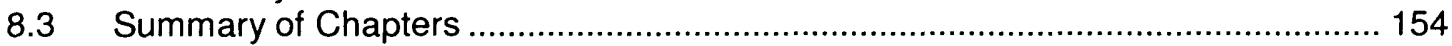

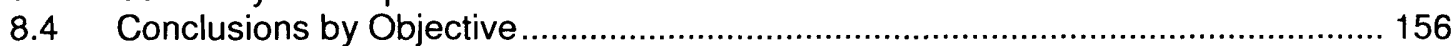

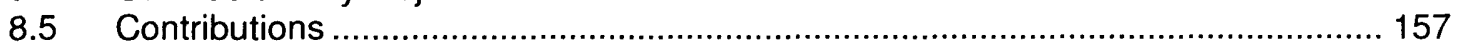

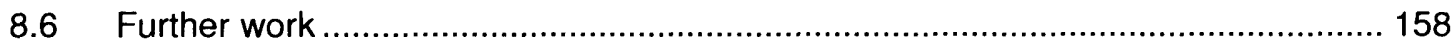

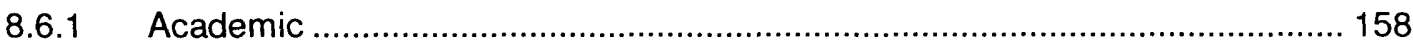

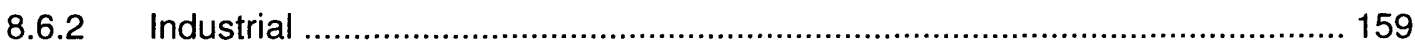

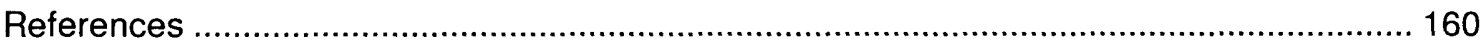

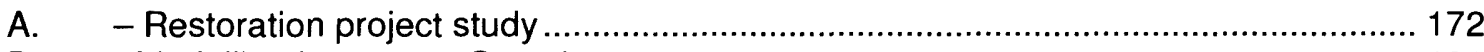

B. - Modelling Language Overview ............................................................... 191 


\section{Table of Figures}

Figure 2-1 - Spiral Model (Boehm 1988) ...............................................10

Figure 2-2 - Risk management (Boehm 1989) ……................................11

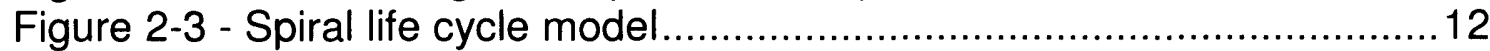

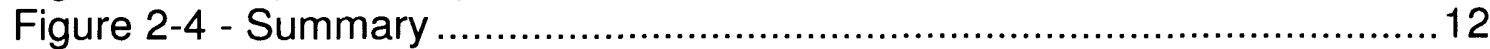

Figure 2-5 - Risk engineering (Hughes and Cotterell 1999) ..........................13

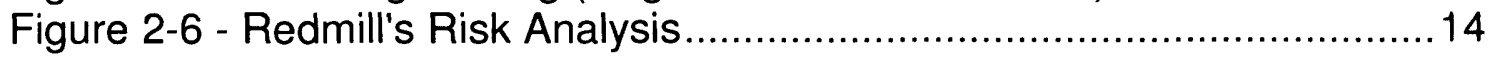

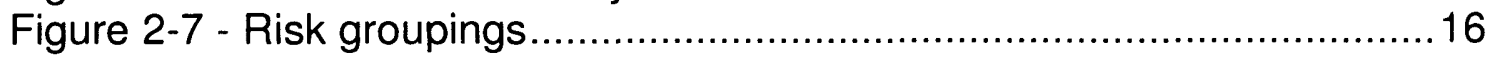

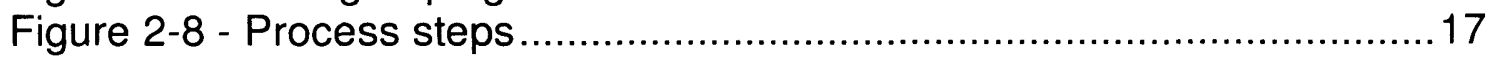

Figure 2-9 - Examples of the Drivers of Key Risks (IRM et al 2002) .............19

Figure 2-10 - The Risk Management Process (IRM et al 2002) .....................20

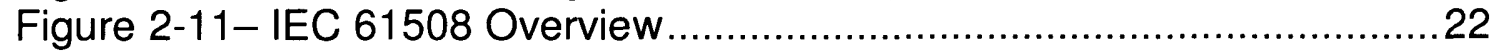

Figure 2-12 - System Life Cycle Stages - Process Structure View ................25

Figure 2-13 - Project - Process Content View.......................................26

Figure 2-14 - AS/NZS 4360 Purpose .....................................................28

Figure 2-15 - Risk Management Process - Overview (AS/NZS 2004) ...........29

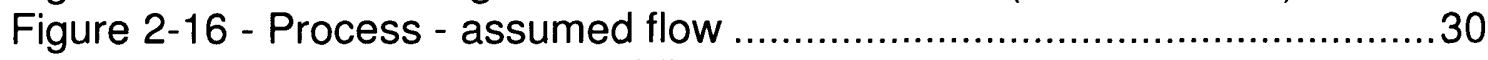

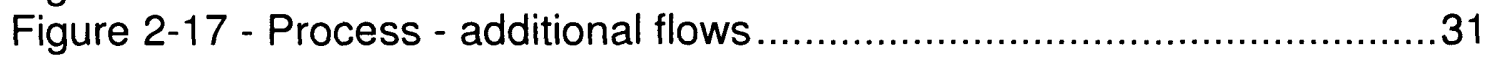

Figure 2-18 - AS/NZS 4360 Terminology Quagmire ..................................32

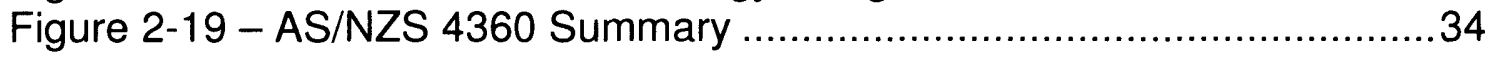

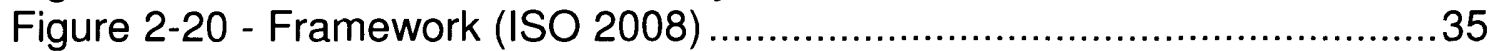

Figure 2-21 - Risk Management Process (ISO 2008) ................................36

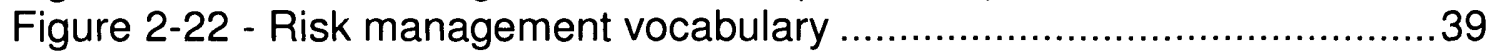

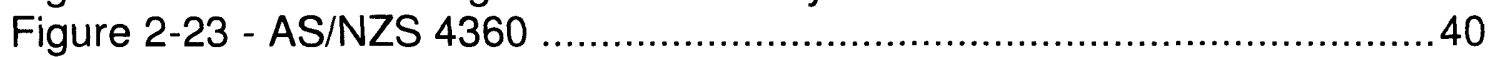

Figure 2-24 - QoS UML profile (OMG 2008b) .......................................42

Figure 2-25 - PHA ontology (Mazouni and Aubry 2007) …..........................43

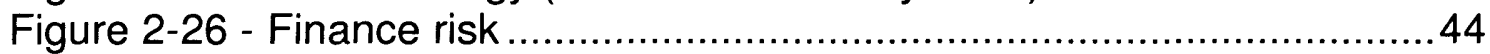

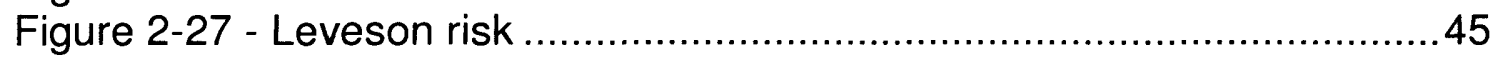

Figure 2-28 - Roland and Moriarty's risk ..............................................45

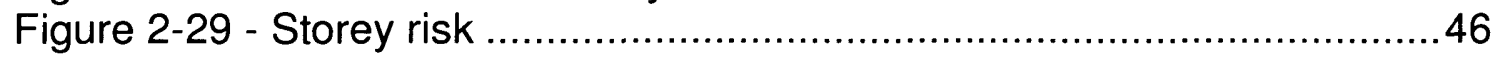

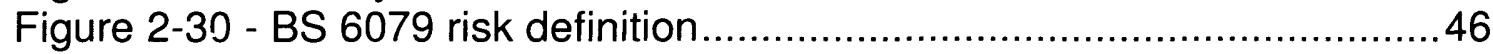

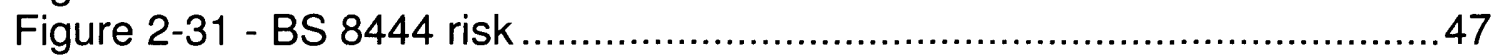

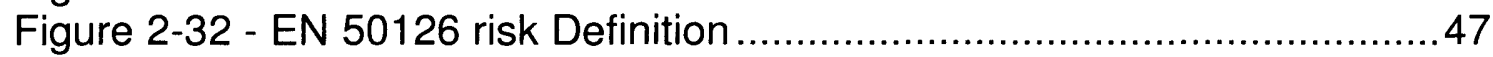

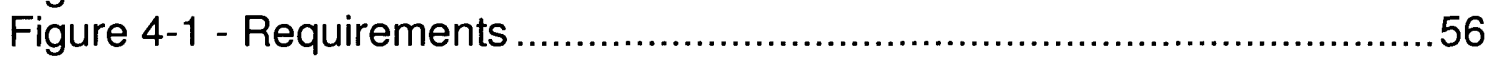

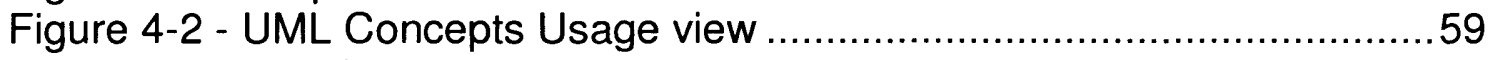

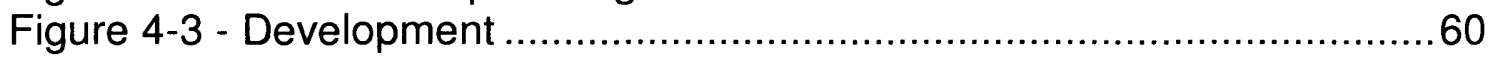

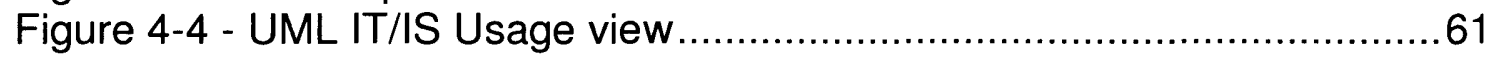

Figure 4-5 - UML Defence Usage view ...................................................63

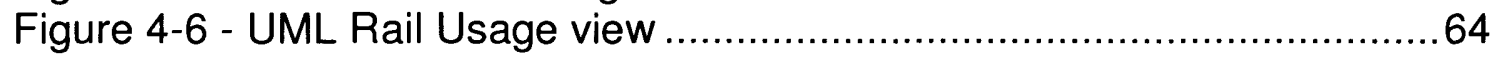

Figure 4-7 - UML Science/Education Usage view.....................................66

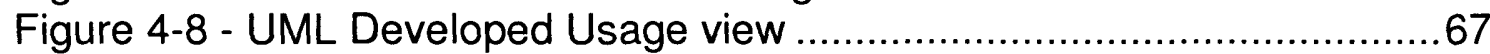

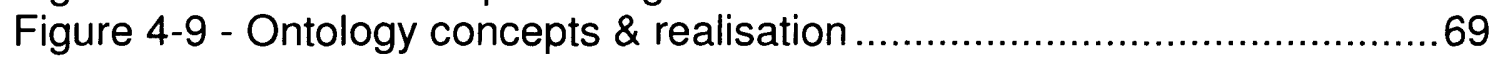

Figure 4-10 - Process Concepts View (Holt 2005) .....................................70

Figure 4-11 - Process Realization View (Holt 2005) ..................................71

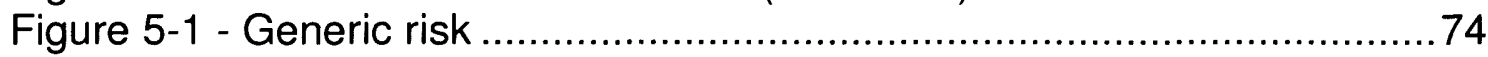

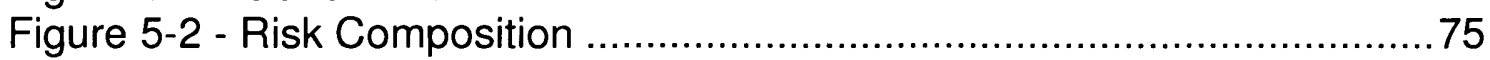

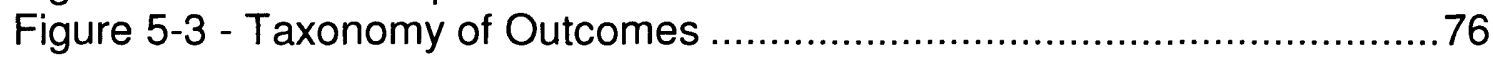

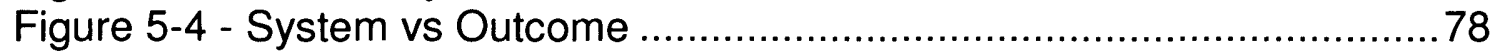

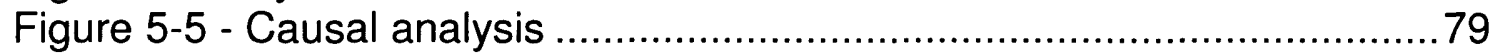




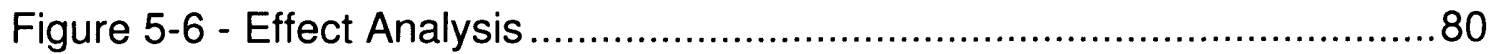

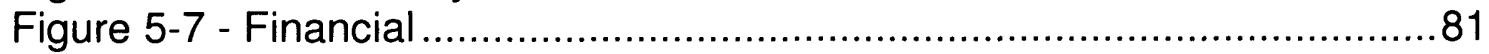

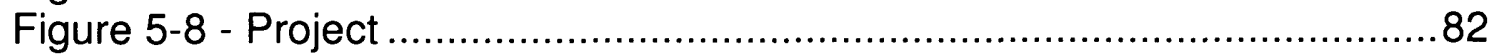

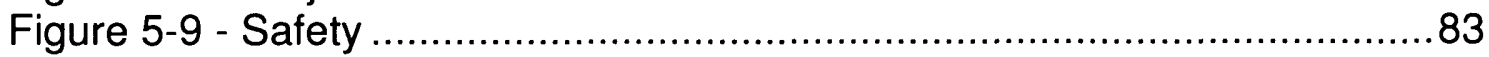

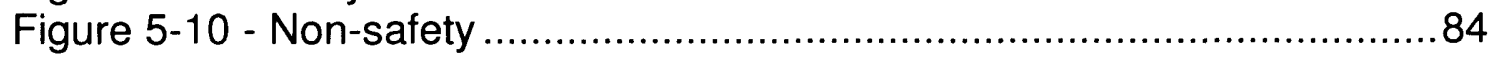

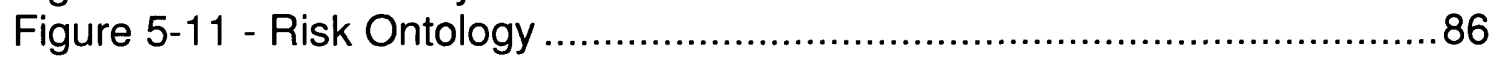

Figure 6-1 - Risk Management Requirements …..................................... 89

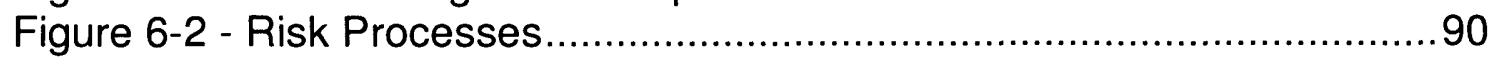

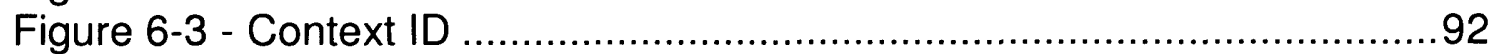

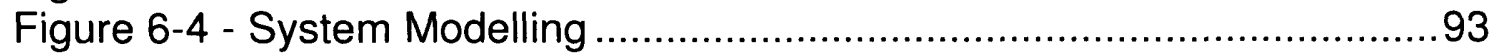

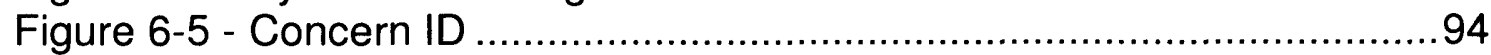

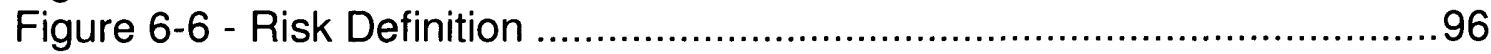

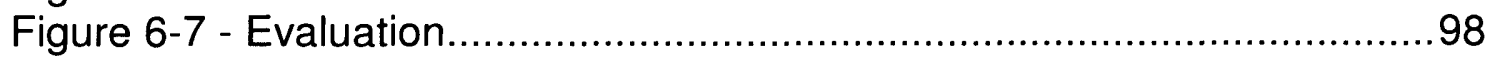

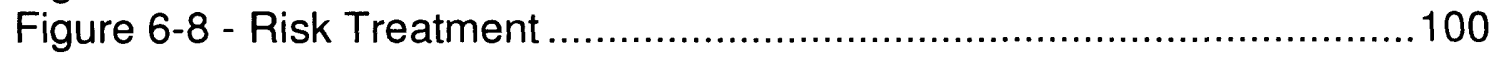

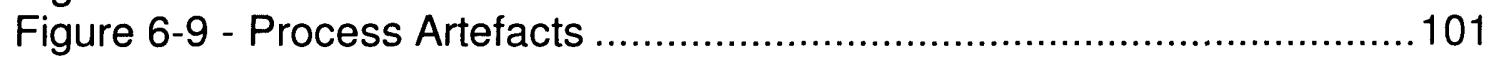

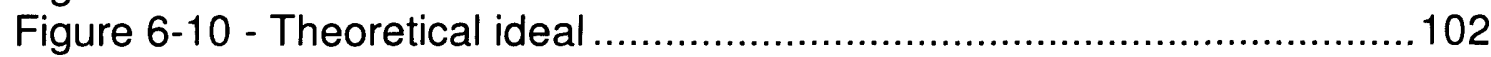

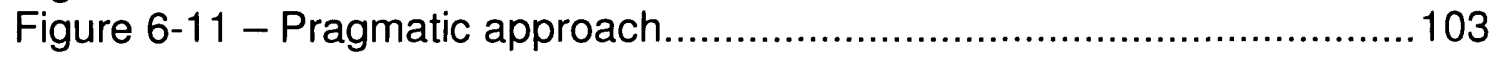

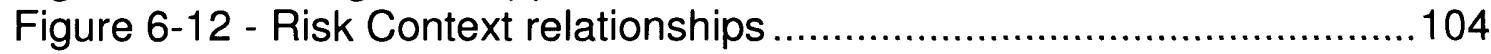

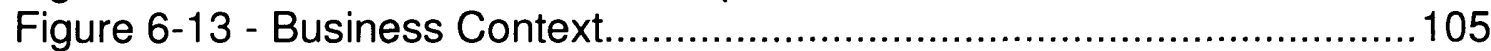

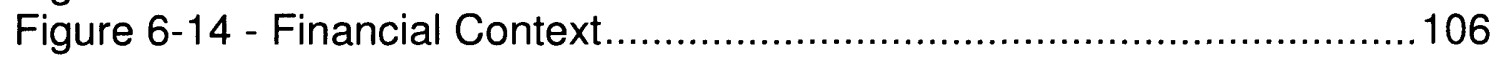

Figure 6-15 - Marketing Context .........................................................107

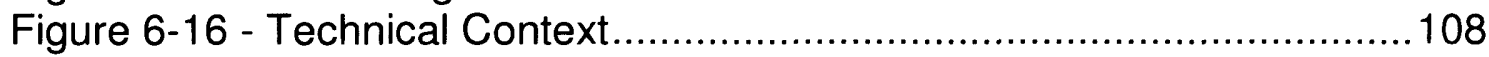

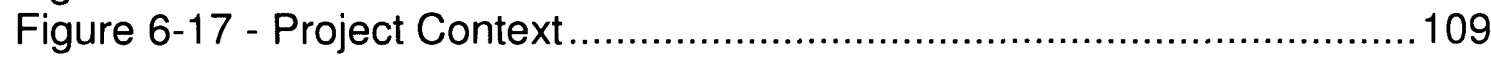

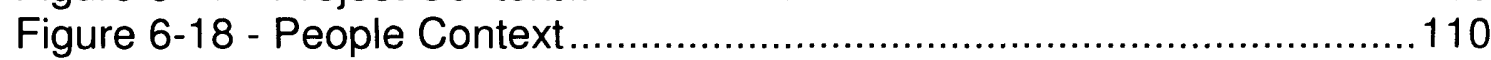

Figure 6-19 - Risk Evaluation example Causal scenario.............................111

Figure 6-20 - Risk Evaluation example Effect scenario..............................112

Figure 6-21 - Risk factor overview ..................................................113

Figure 6-22 - Risk Ontology with processes .........................................114

Figure 7-1 - Initial Process Instance View...............................................117

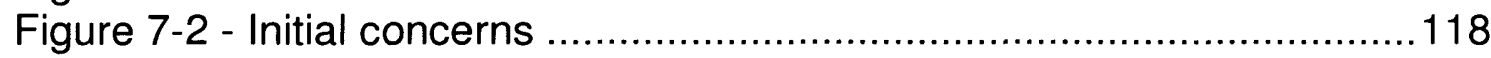

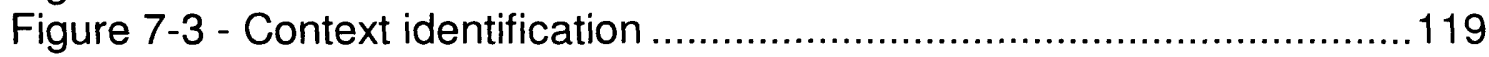

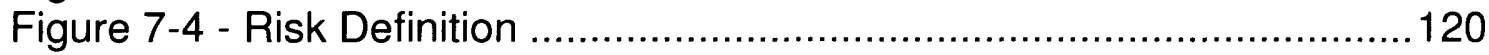

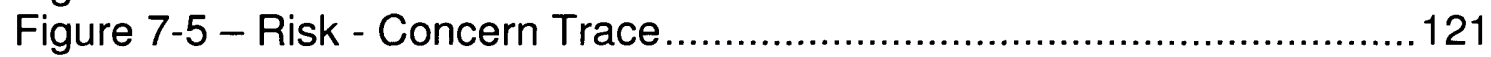

Figure 7-6 - Scalding child Process Instance View ..................................122

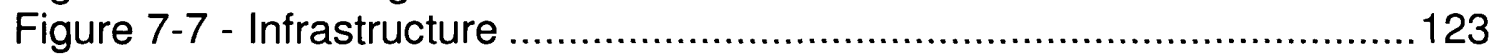

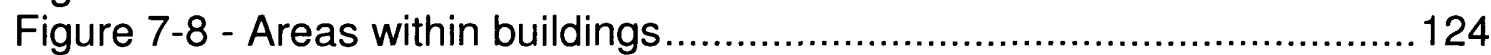

Figure 7-9 - Population - St Nicholas Urn Concern ...................................124

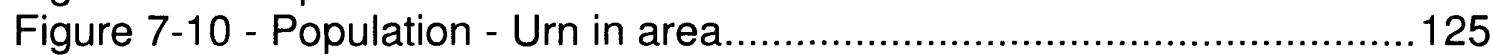

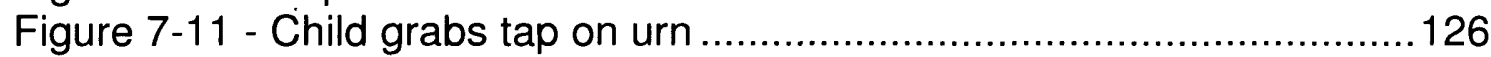

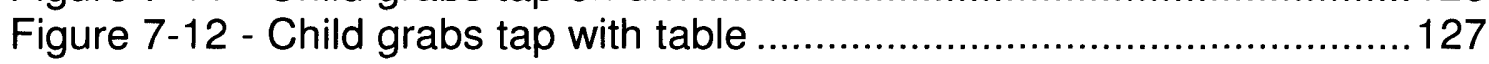

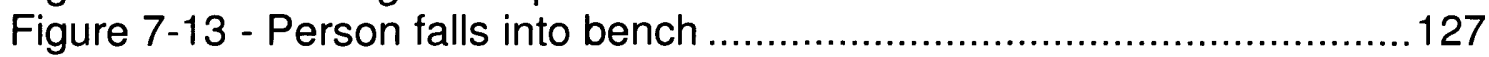

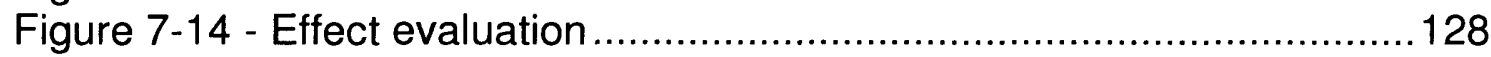

Figure 7-15 - Risk Treatment - Scalded child - Solution 4 ........................130

Figure 7-16 - Risk Treatment - Scalded child - Solution 7 7.......................131

Figure 7-17 - Treatment - Scalded child.............................................131

Figure 7-18 - Concern Identification Revisited …….............................132

Figure 7-19 - Parish review methodology ............................................... 134

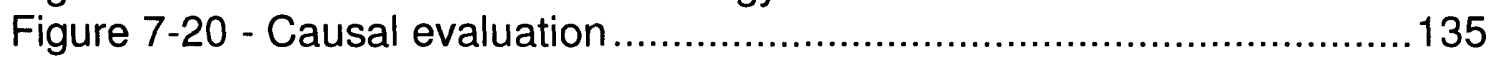

Figure 7-21 - Risk Treatment - No new clergy ......................................136

Figure 7-22 - Risk Treatment - No new clergy ......................................137 
Figure 7-23 - Project actual Process Instance View................................138

Figure 7-24 - Tailored project context ...................................................140

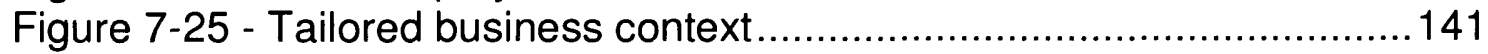

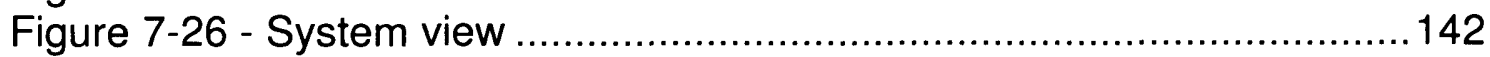

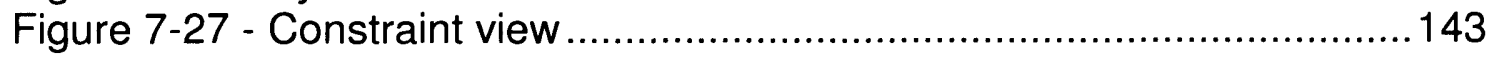

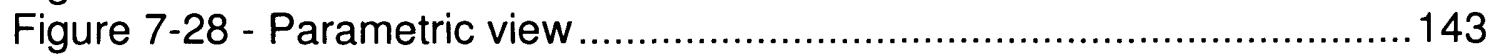

Figure 7-29 - Personal Development .................................................. 145

Figure 7-30 - Competence .............................................................147

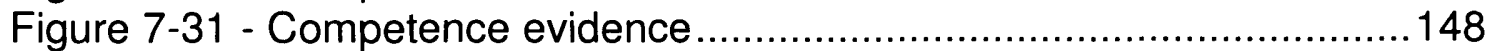

Figure 7-32 - standard - work - competence ........................................148

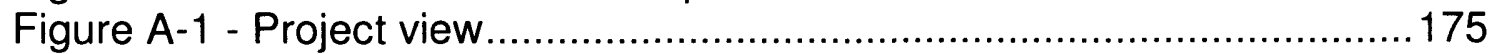

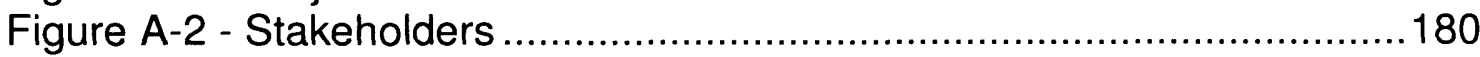

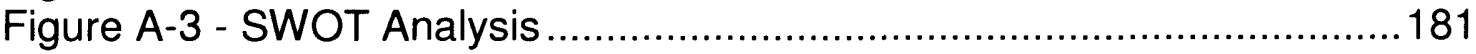

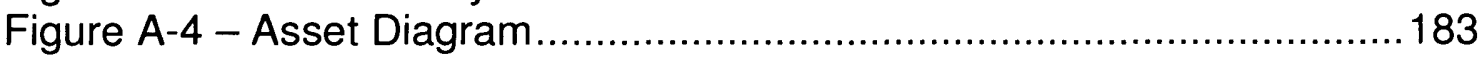

Figure A-5 - Threat Scenario 'Bad Weather' .........................................186

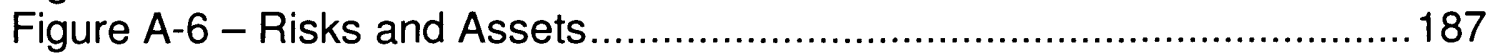

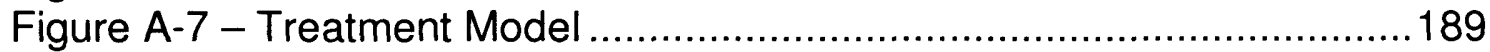

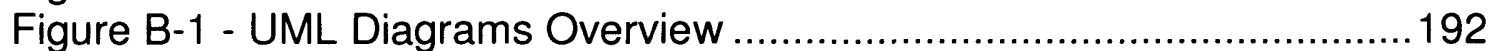

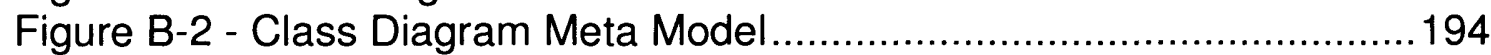

Figure B-3 - Class Diagram Symbols.................................................195

Figure B-4 - Example structure .......................................................196

Figure B-5 - Example classification .................................................196

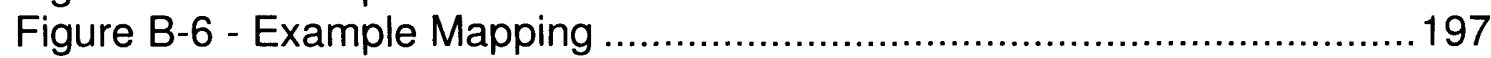

Figure B-7 - Class Relationships Overview...........................................198

Figure B-8 - Activity Diagram Meta Model ............................................199

Figure B-9 - Activity Diagram Symbols …….......................................199

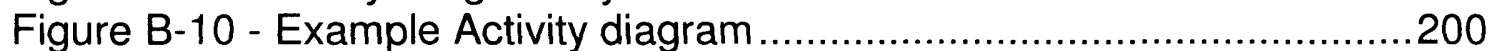

Figure B-11 - Deployment Diagram Meta Model....................................201

Figure B-12 - Deployment Diagram Symbols ........................................201

Figure B-13 - Example Deployment ...................................................202

Figure B-14 - Sequence Diagram Meta Model......................................203

Figure B-15 - Sequence Diagram Symbols...........................................203

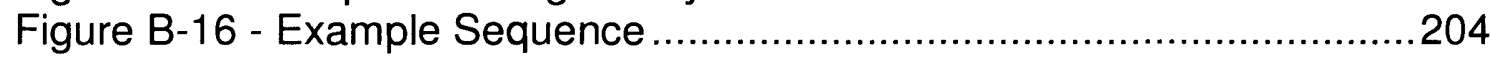

Figure B-17 - Use Case Diagram Meta Model ........................................205

Figure B-18 - Use Case Diagram Symbols ……….................................206

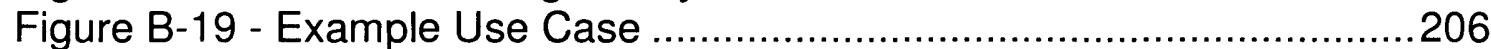

Figure B-20 - UML Diagram Relationships ......................................207

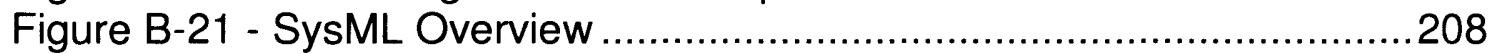

Figure B-22 - Parametrics - Definition Notation .....................................209

Figure B-23 - Parametrics - Usage Notation .........................................210

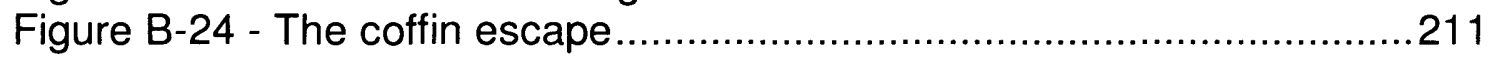

Figure B-25 - The coffin escape - parametric definitions..........................212

Figure B-26 - The coffin escape - parametric usage ..............................212

Figure B-27 - SysML Diagram Relationships ......................................214 


\section{Introduction}

\subsection{Background}

The consideration of risk is a day-to-day phenomenon used by individuals, Small to Medium Enterprises (SME's) to large national, multinational or global organisations. Although in many instances risks may be 'mitigated' this does not mean that complex issues have been well understood.

Risk Management proposes to be a solution to understanding and removing the worry associated with issues which may arise in the future. As a discipline Risk Management has existed since the 1960's emerging from an historic need and desire to insure. From the 1980's clear reference can be made to a process for risk management which has remained relatively unchanged.

There are many tools available to assist in the modelling of complex systems. Modelling allows simplification of the system to allow the complexity to be understood or at least to aid the recognition that there is a complex issue. These tools vary from high level business strategy identification to Failure Modes and Effects Analysis (FMEA) examining the detail associated with failures of components in a system.

Risk can be a very personal thing; people generally understand risk in slightly different ways due to their own experiences. This view of risk is not directly associated with numbers, probabilities and specific outcomes. It is about the chance that the individual may lose something of value. The situations where people think about risk are around us all the time from investments to flying or crossing the road to bungee jumping. The question is how do people think about risk? Generally as life progresses new challenges arise, situations which have not been encountered previously. In the case of these new situations people tend toward caution, taking things slowly trying to ensure the best outcome. Once the situation has been tried and tested confidence grows and it is possible to start to believe that there is no danger or risk as the situation has been encountered many times and has always ended well. 
People are happy to build up these perceptions about their situations although the perceptions can be changed in an instant. No relationship between the mind changing event and the situation need exist. For example after September 11th 2001 the perception of safety of air travel changed dramatically.

There is an inherent psychological impact on the way people understand risk however this psychology is outside the scope of this work.

Many industries recognise risk and the need for risk management. The railway industry for example has a defined and documented regime for addressing risk. This regime is documented and controlled through the use of standards such as EN 50126:1999 (CENELEC 1999) Railway applications The specification and demonstration of Reliability, Availability, Maintainability and Safety (RAMS). RAMS introduces risk as a safety concept within the standard which can be seen to run throughout a project lifecycle.

However many industries have not recognised either the importance of formalising risk management and the surrounding issues or that the technology they are working with has associated risk. This lack of recognition can impact many dimensions including legal, personal and technological.

\subsection{Context/Scope}

Observations made whilst working with Aerospace, Rail, Defence and Government organisations have shown a number of issues with the implementation of current risk management best practice. In some cases these issues arise due to a lack of willingness to carry out thorough risk management or to react when risks are revealed. However in many cases these managerial issues are compounded by fundamental issues of complexity and lack of pragmatism associated with the risk management process. Inhibiting the resolutions of many of these issues is the lack of understanding and agreement on terminology used to describe and discuss risk. 
Issues include:

- consideration of risk management as a support process,

- differing desires of stakeholders,

- the need to re-create existing documents or designs to enable risk management to be carried out,

- the multitude of possible routes through the risk management process and

- the variation in meaning associated with the word 'Risk' and other risk management terminology

The view of risk management as a support process causes issues as there is a view that support processes are add-on processes armoured in documentation which provide no real purpose other than to delay the project until after the prescribed deadline.

There may be a number of reasons for the image associated with these support processes. One may be that they are in fact much more integral to the work than most would like to admit. As such many of the activities are carried out as a matter of course. In relation to these activities the risk management process duplicates work adding rather than removing the likelihood of errors or failure.

Not only can the process duplicate work already carried out it can also deliver an inconsistent view of the order in which the risk management steps should or could be carried out. The process is generally wrapped in catchall activities relating to all steps ensuring that no assumed flow can be guaranteed.

All of these issues stand in the way of considering the information regarding a 'risk' which as a word means something different almost every time it is used. 


\subsubsection{Formalisation}

These issues, duplication of information, complexity of process and incoherence of terminology, drive the need for a more formalised approach to risk management. To ensure that complexity is reduced rather than increased a tried and tested method of understanding complex, diverse and multifaceted systems must be applied.

Formalisation, in this work, means to emphasise the logical approach taken towards the understanding and definition of risks. This approach in turn supports the validity of the risk. Within the current engineering and economic climate this logical approach must provide a visualisation which is consistent, repeatable and view based and pragmatic.

The software/IT domain has been using these kinds of formalised techniques for many years. In the last 15 years there has been a move to provide consistency across these techniques. One of the results of this need for consistency is the Unified Modelling Language (UML). The UML has been used in many applications from project management to real-time critical system design making use of its syntax, ability to communicate and provide consistency both internally, between visualisations and externally through links to real examples and scenarios.

As the UML provides a consistent, graphical multi-view approach to understanding complex, diverse and multi-faceted systems its application to the concepts of risk enable a consistency, formalisation and visual representation of the terminology and process of risk management. Many of the diagrams, shown using the UML, within this work provide interpretations of the original work rather than the work itself, where this is not the case full references are provided. These interpretations, as well as providing a consistent understanding of diverse works, provide an independent view of the work enabling an overview to be gained and faster access to relevant areas within the work. 
Having a consistent, formalised approach supports repeatability within a formalised approach by focusing on modularisation and re-use without forgetting the overall need $s$ of the whole.

The use of modularisation within risk management enables the pragmatic application of the processes required to carry out risk management. Pragmatic application is achieved through the timely execution of relevant processes, this may mean that one processes is carried out many times whilst another is only used once or twice. The pragmatic application of the process has the added advantage of improving the accuracy of project plans and records.

There are three main areas which must be addressed prior to the application of a formalised approach these areas are the ontology, processes and methodology.

- The ontology provides the definitions of terms, as per a glossary or dictionary, and well defined relationships between the terms (which is lacking in many cases).

- Each process provides a concise set of activities to be carried out showing which artefacts, documents or information, are consumed and produced by each activity. Relationships between artefacts are also defined.

- The methodology provides the ordering for application of the processes. This ordering can show planned or actual ordering enabling consideration and improvement in planning.

There are two key long term benefits to a formalised approach to risk management which are:

1. Improved application of risk management on current and future projects

2. Improved education for those who will apply risk management enabling further improvements in 1. 


\subsection{Objectives}

The primary aim of this thesis is to develop a formalised approach to the management of risk using a model based approach. This will address the problem of a lack of formalisation across risk management in many industries, applications and education.

To achieve the overall aim both terminology and processes related to risk must be understood and formalised. Formalised ontology and processes will be developed and demonstrated through a number of threads abstracted from a case study.

The aims and objectives outlined here will be addressed throughout the thesis; the main contribution of each chapter to these objectives is:

- Chapter 2 - to review and understand the terminology and processes related to risk management.

- Chapter 3 - to present the methodology for the research.

- Chapter 4 - to discuss the requirements for a model based framework for the formalisation of risk management and propose a framework which fulfils the requirements.

- Chapter 5 - to define risk and present an ontology for risk management.

- Chapter 6 - to define the processes required to carry out risk management using a multi-view approach.

- Chapter 7 - to demonstrate the applicability of the processes and ontology. 


\subsection{Thesis plan and structure}

This thesis makes extensive use of the Unified Modelling Language (UML) as an approach to understanding and describing concepts and systems. The approach is born out of the Object Oriented (OO) world of systems understanding and can be used to describe system needs, structure, hierarchy, state and activity at many different viewpoints and levels of abstraction. It is not, however, the intention here to provide a detailed survey of UML, but brief introduction to the notation is given in Appendix B. Further detailed references are found in Stevens and Pooley (2005), Holt (2005) and Holt (2008).

The following gives a more general overview of the thesis defining what is discussed in each chapter:

This chapter introduces the need for this work covering complexity, risk related to people, industry and standards. It defines the aims and objectives of the work and provides an overview of the thesis

Chapter two investigates the processes defined for risk management, the terminology defined along side these processes and tools and techniques used to examine and create the data required to understand risks.

Chapter three presents the methodology for the research considering the use of empirical vs theoretical and phenomenological vs positivist approaches.

Chapter four discusses the use of the Unified Modelling Language (UML) as a visual, multi-view modelling approach and shows that this language is a relevant language to use for understanding and implementing risk management and analysis. It does this through defining the requirements for the notation to be used and investigating a number of applications of the UML.

Chapter five presents the generic terminology of risk and the relationships between the terms, by way of an ontology. Sections of the ontology are 
compared with definitions and terminology discussed in chapter two.

Chapter six presents the set of processes which can be used to manage risk, it presents a number of views of these processes giving confidence that they have been fully defined. This is supported by a set of examples showing possible outputs from the processes. This chapter also defines a methodology providing theoretical and practical application sequences for the processes.

Chapter seven presents two examples, from a case study, of the application of the ontology defined in chapter 4 and the processes defined in chapter 5 to verify the processes and ontology.

Chapter eight presents conclusions drawn from the project as a whole with recommendations for areas of further work. 


\section{Literature Review}

\subsection{Introduction}

The objective of this chapter is to provide the background understanding of risk, which will be achieved by reviewing relevant literature. Much of the literature relating to risk management is specific industry, application and tool. This work is focused on the process of risk management and associated terminology, therefore the literature in this review will be considered in three groups, those relating to the process, terminology and tools/techniques for risk management

Process based literature will be reviewed exploring empirical and standardised approaches to risk management. Approaches based on standards will be used to give a baseline or current best practice in risk management, tools and techniques will be discussed in order to understand their relevance and place within risk management. As well as considering terminology through each piece of literature a focused discussion on overall set of terminology will be given with a view to defining a consistent understanding and set of terminology to provide a foundation for a successful risk management approach.

\subsection{Process}

There are many understandings of the term process. This section focuses on understanding what risk experts and standards mean when they discuss the 'Risk Management Process'. The focus will be on those authors who have defined a process and standards which present a baseline approach to risk management.

In his tutorial on software risk management Boehm (1989) presents a number of steps which are aimed at identifying, addressing and eliminating software risks before they cause re-work or failure. This work was carried out when software risk management was considered to be an emerging discipline, however many of the concepts are still applied. 
Boehm has defined both the steps for risk management and a life cycle 'The spiral model' (Boehm 1988) in which the steps can be applied. The spiral model evolved over a number of years. It is based on the waterfall model with a number of refinements applied to it.

This model is defined to provide an approach to development which is risk driven and reflects the incremental nature of most development projects.

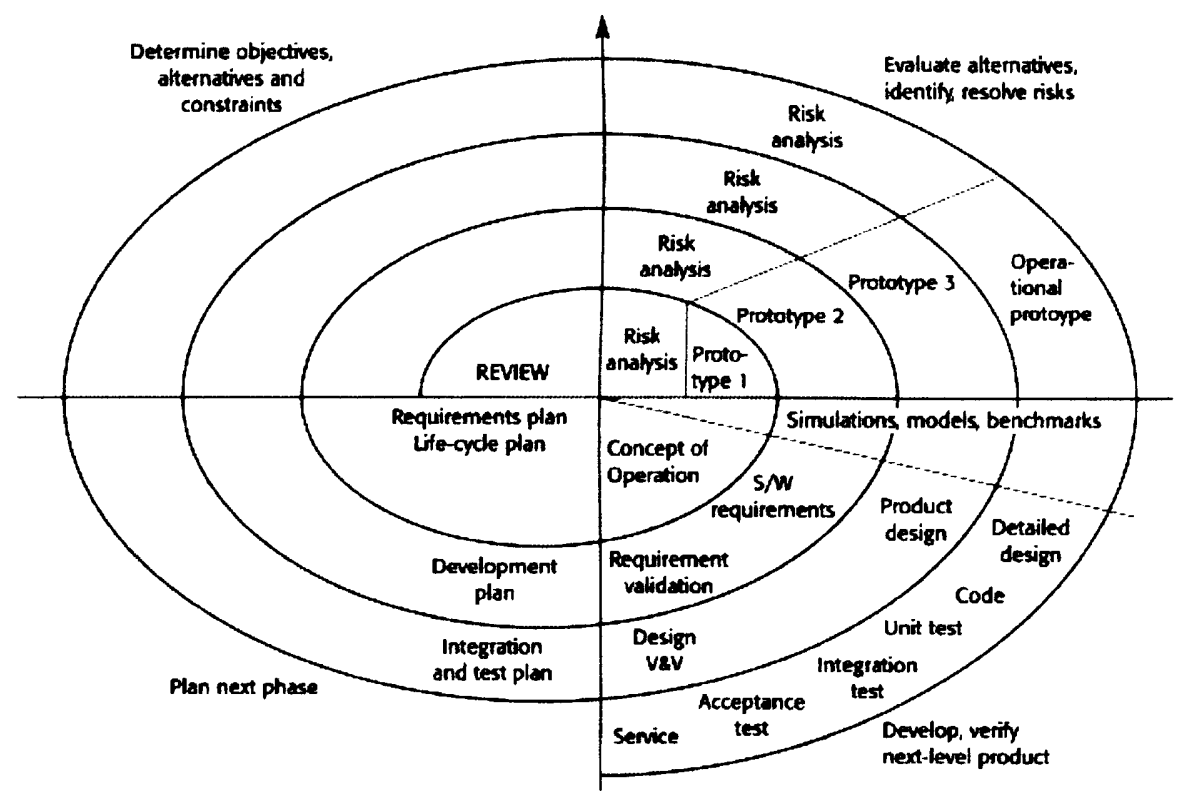

Figure 2-1 - Spiral Model (Boehm 1988)

The spiral model (Figure 2-1) provides an incremental approach to defining requirements, architecture and design through the re-use of the four main elements 'Determine objectives, alternatives, constraints', 'Evaluate alternatives; identify, resolve risks', 'Develop, verify next level product' and 'Plan next phases'. There are further refinements and detail provided within each phase or element and a revised spiral model was also produced expanding on the detail within the 'Plan next phases' element.

Boehm (1989) states that "Software Risk management is an emerging discipline whose objectives are to identify, address, and eliminate software 
risk items before they become either threats to successful software operation or major sources of software rework." he provides steps to support these objectives.

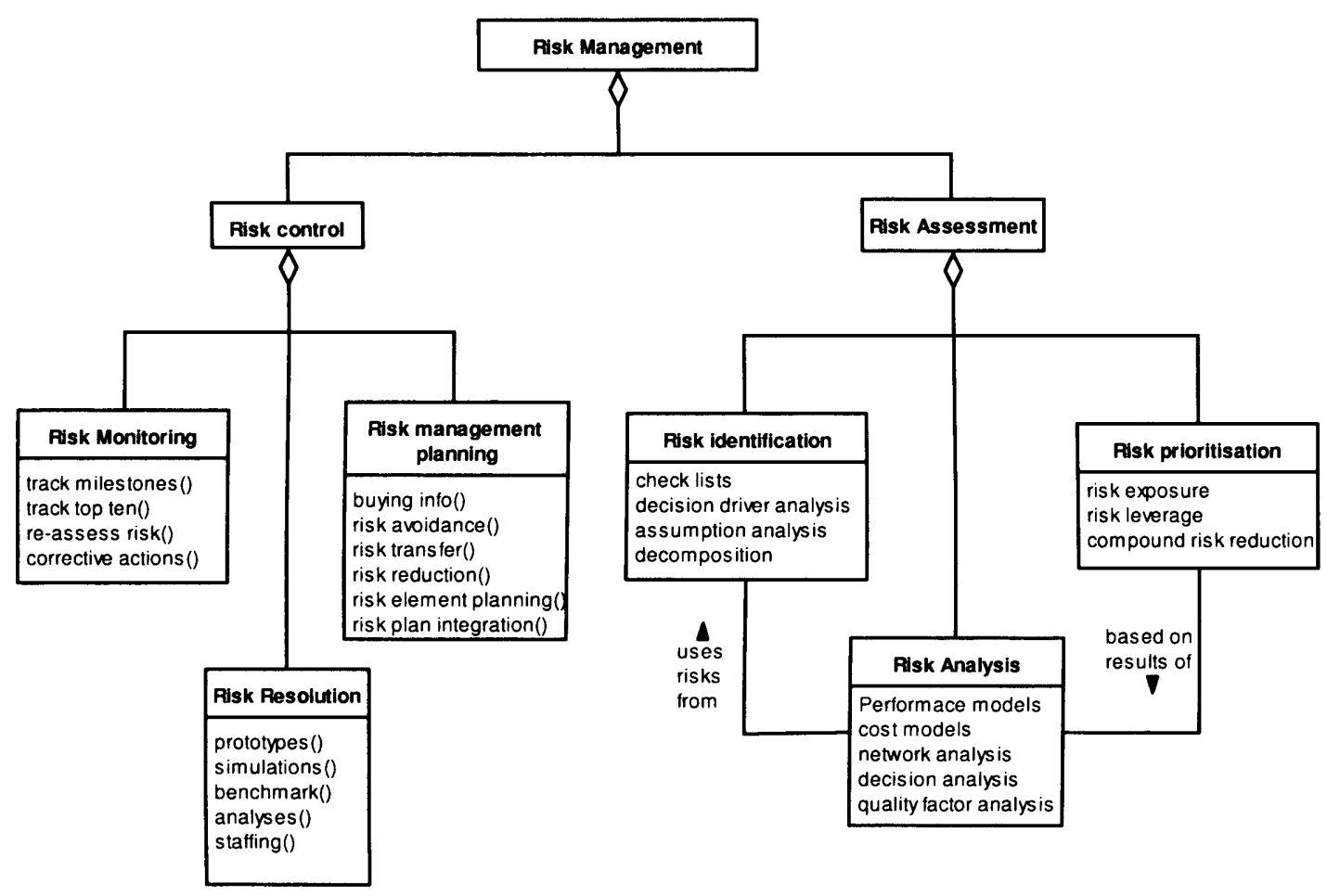

Figure 2-2 - Risk management (Boehm 1989)

Boehm defines two primary steps (Figure 2-2), within risk management, of risk control and risk assessment. Three sub-steps exist within each; risk control covering management, monitoring and resolution and risk assessment involving identification, analysis and prioritisation. These steps are further defined with some explanation of the items which may be produced by the step as well as typical techniques.

The disadvantage of the spiral model, this works interpretation of which is abstracted in Figure 2-3, is that it specifies the work to be completed in each step: this constrains the flexibility of the model and therefore its application to other areas. Another way of explaining this would be to say that it begins to pre-define the project plan. 


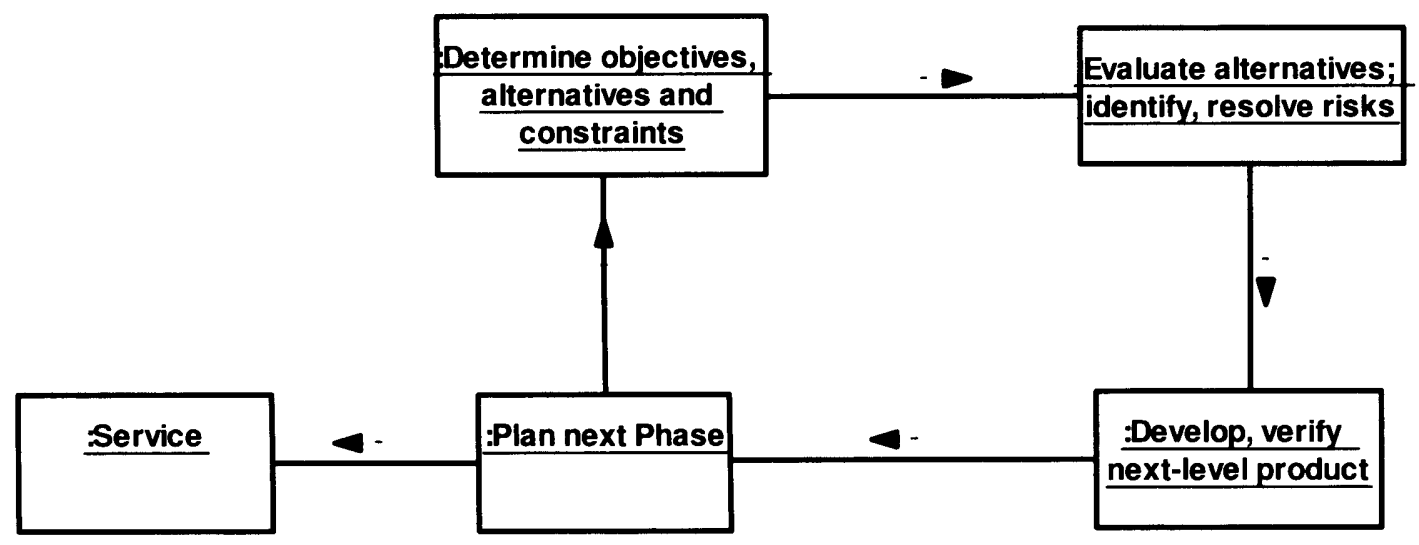

Figure 2-3 - Spiral life cycle model

Boehm has provided a differentiation between the management, which he called control, in 'risk management' and the assessment in 'risk assessment' providing in many cases a useful delineation between the work of identifying and fully defining risks and the plans and controls which need to be in place to ensure that risks are dealt with effectively.

Together the spiral model and risk management steps provide an approach for the business to incorporate risk management into projects (Figure 2-4)

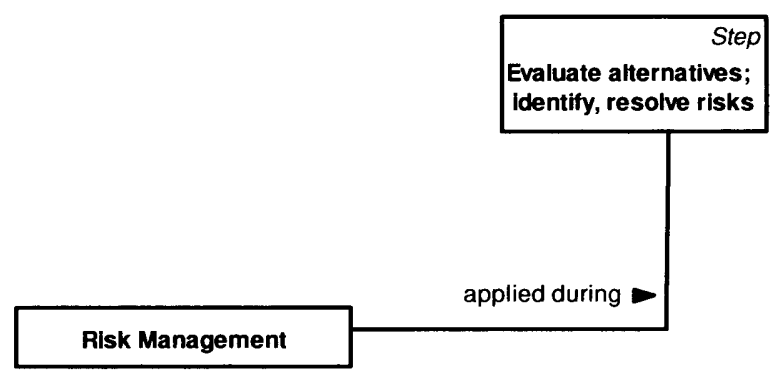

Figure 2-4 - Summary

Overall Boehm's model provides a sensible way forward when addressing risk in the software environment giving a general overview from a management or project perspective.

Lee et al (2009) use Boehm's work with others to generalise risk management into four phases: classification and identification, risk assessment, risk analysis and risk control. Lee applies Bayesian Belief networks (Aven 2003) 
within the phases incorporating uncertainty into risk management. Although some technical risks are identified the majority of the work is focused on management issues associated with the company and project rather than the impact of technical issues.

Hughes and Cotterell (1999) have extended Boehm's model of risk management, starting with a re-partitioning of the risk management steps (Figure 2-5).

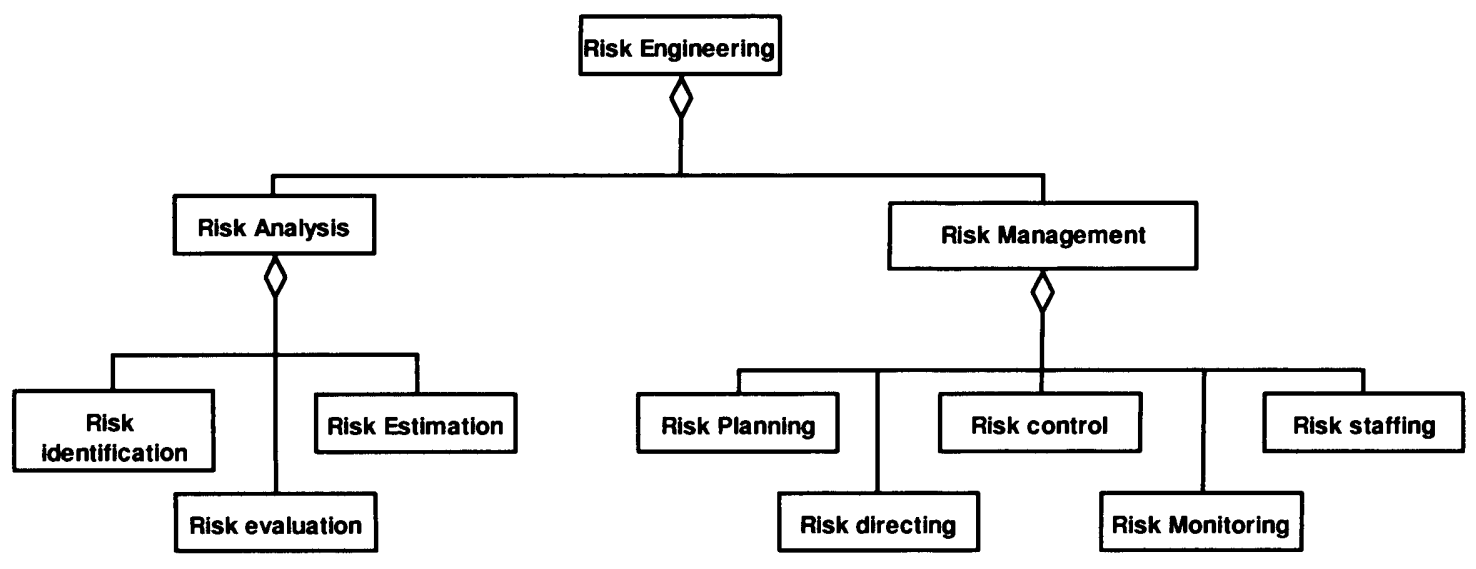

Figure 2-5 - Risk engineering (Hughes and Cotterell 1999)

They also provide two primary areas within risk; Risk Analysis and Risk Management. The management area groups all planning, staffing, directing, monitoring and control activities whilst the Analysis area focuses on the identification, estimation and evaluation of risk.

Re-sectioning Boehm's risk management steps separates the analysis of risk from its management whereas Boehm's model looked at assessment and control. It is believed that many of the changes are in the meaning of the word 'management'. In Boehm's work the use of 'risk management' was used to signify the whole area of risk assessment, analysis and control whereas Hughes and Cotterell are using management to specify only the control, planning and resource issues leaving identification, estimation and evaluation being grouped as Risk Analysis.

It is unclear in this model how the identification, estimation and evaluation will 
be carried out, where the techniques to be used will be selected, again the focus is on the management of risk rather than the definition and analysis of technical risk. Kirchsteiger (2008) takes a similar view when he explains risk assessment as the 'fact finding' and administrative follow up measures as risk management.

Redmill (2002) highlights the three stages most consider to be included in risk analysis, hazard identification, hazard analysis and risk assessment (or evaluation). He goes on to expand these steps by adding a 'definition of scope', highlighted in Figure 2-6, concerned with the planning of the work to be carried out during the risk analysis. Jenkins et al (2009), Mohaghegh et al (2009) and Olsen and Lindoe (2008) all use the concept of context or viewpoints within their work. Olsen and Lindoe use context to understand the implications of transferring technology between contexts. Jenkins et al use dimensions to develop a management framework and Mohaghegh uses perspectives and multilevel framing to ensure the relevant aspects are included in the analysis.

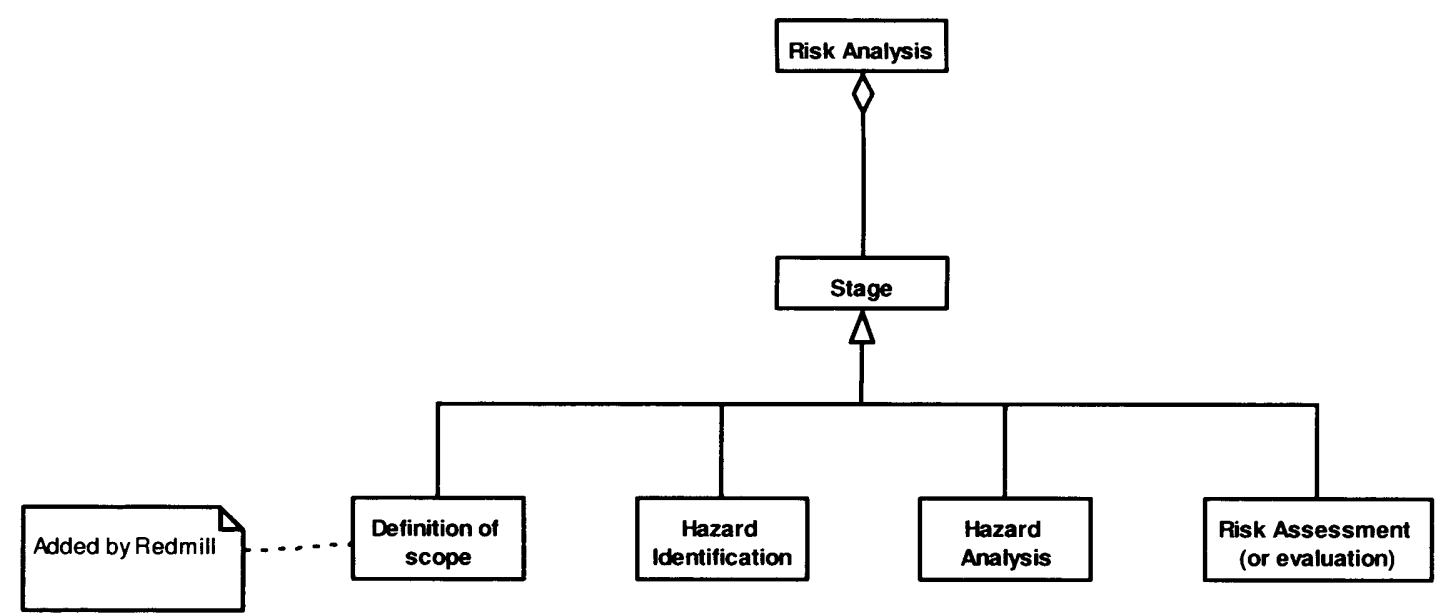

Figure 2-6 - Redmill's Risk Analysis

Further to each of these stages Redmill discusses many of the tools which can be used within these stages to carry out the detailed work required, he uses this to highlight the areas where subjectivity can enter into the provision of a full analysis for a project or product. 
Redmill's work from 2002 seems to lack the breadth and depth of definition found in Boehms work from 1989. This lack of consistency is symptomatic of the lack of connectivity across disciplines when it comes to the understanding of Risk.

It is useful that he reflects on the issues from project management in terms of cost and time in relation to the detail required from a technique used to define hazards within the risk analysis stages.

It is also noticeable that Redmill is predominantly concerned with hazards or causes and that risk is the end point of understanding hazards where others including Boehm (1989) the risk process has been focused on effects rather than causes. Woodruff (2005) argues that the focus on consequence and hazard ensure that decisions are not based on overall risk which he suggests is leading to risk averse stakeholders defining the level of acceptable or tolerable risk.

Redmill's work seems to be behind the mark in relation to Boehm however this may be due to the relative maturity of risk management across different industries. The benefit Redmill adds is by ensuring that the scope of the assessment is defined.

The variation in definition between Boehm and Redmill, including the focus on cause or effect, highlights a need to consider standard practice. It will be important to note whether the standards are industry specific or cross discipline.

\subsection{Standards}

Mainly concerned with the approach to Risk Management numerous standards can be consulted to see what is claimed to be best practice. Standards may be company, national, or international some of which are used as much outside the expected boundary as with AS-NZS 4360 (2004) an Australian standard which is recognised in Canada by the Canadian Information Processing Society (CIPS) Risk Management document (CIPS 2007), mainland Europe through European projects such as CORAS (Lund et al 2004) and in the UK within the Fishing industry. 
There are many differing views on what constitutes risk management, analysis and assessment hence the need for standardisation across industries and domains. This section will investigate whether standards have provided a common understanding and approach to risk management.

Many professional bodies and industry organisations provide guidance, codes of practice and principles for risk management. These include the Institute of chartered accountants in England and Wales (ICAEW) (2002), the Institute of Risk Management (IRM) with the Association of Insurance and Risk Managers (AIRMIC) (2002) and CIPS (2007) to name a few.

ICAEW in its briefing on Risk management for SMEs (2002) identifies the need to apply risk management across the organisation expanding from the previous narrow financial view it took.

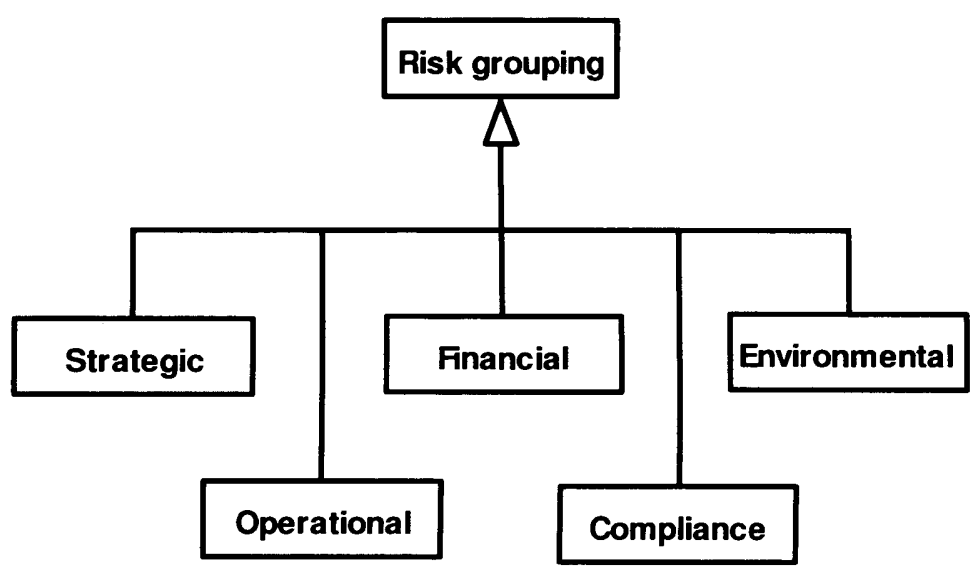

Figure 2-7 - Risk groupings

The briefing goes on to describe five headings, described in Figure 2-7, under which risk maybe grouped or assessed. The groupings are;

- Strategic - risks from industry or geographically areas,

- Operational - risks from operations and administrative procedures,

- Financial - risks from the financial structure and third party transactions,

- Compliance - risks from law and regulation including Health and Safety,

- Environmental - risks could be covered under compliance. 


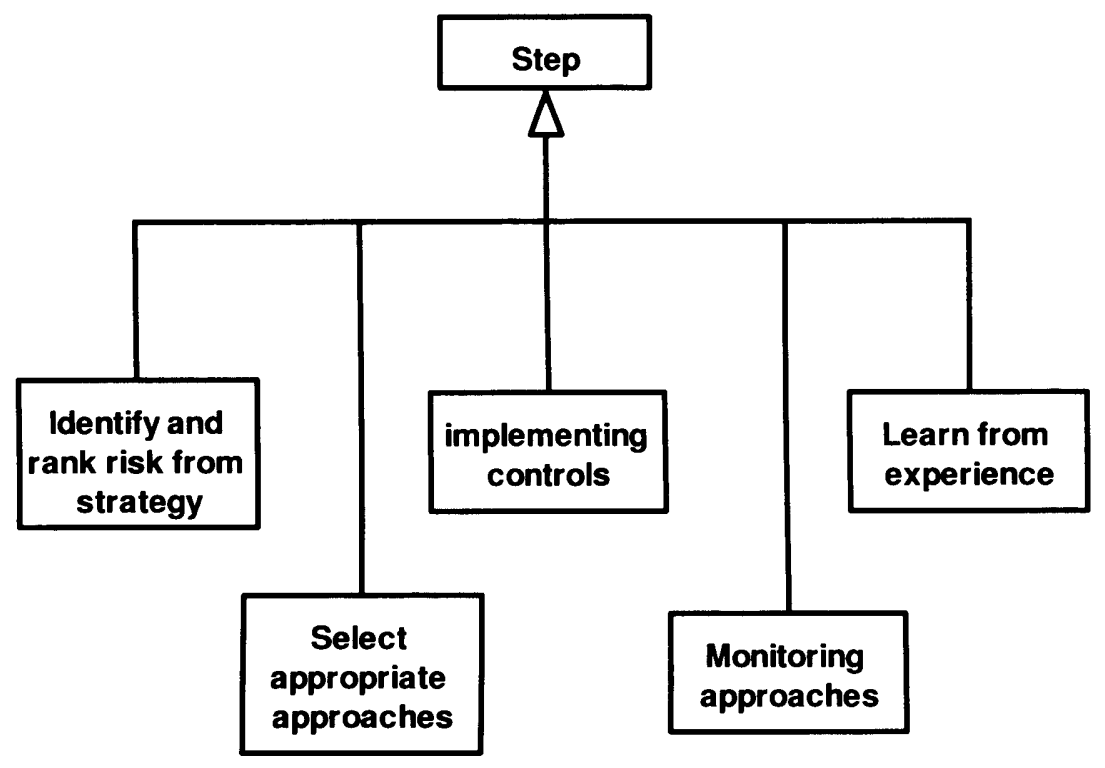

Figure 2-8 - Process steps

The briefing goes on to state that the risk management process will normally involve the following steps

- Identifying and ranking the risks inherent in the company's strategy (including its overall goals and appetite for risk);

- selecting the appropriate risk management approaches and transferring or avoiding those risks that the business is not competent or willing to manage;

- implementing controls to manage the remaining risks;

- monitoring the effectiveness of risk management approaches and controls; and

- learning from experience and making improvements.

Following this it suggests four ways of controlling risks known as the four T's; tolerate, transfer, treat or terminate.

Expressing that risk should be cross business rather than purely financial is a step in the right direction but it misses the opportunity to explain whether 'business' is only the management side of an organisation or whether it is 
intended to cover the whole organisation including technical project and development. It is limited in its consideration of these areas when applying the categories of risk, shown in Figure 2-7, it has defined.

As a briefing document this is forward thinking and open, providing a good basis for improvement. However, the similarity between the risk management requirements and process means that the standard misses the opportunity to explain or justify why risk management should be carried out, whether it is a core business process or not, it only succeeds in defining the activities that the process must deliver.

The IRM et al (2002) have defined 'A risk management standard' which it is stated "is the result of a team drawn from the major risk management organisation in the UK" The standards sets out risk management as "a rapidly developing discipline" which has a need for a standard to ensure that there is an agreed:

- terminology related to the words used

- process by which risk management can be carried out

- organisation structure for risk management

- objective for risk management

The standard begins by defining the drivers of risks. 


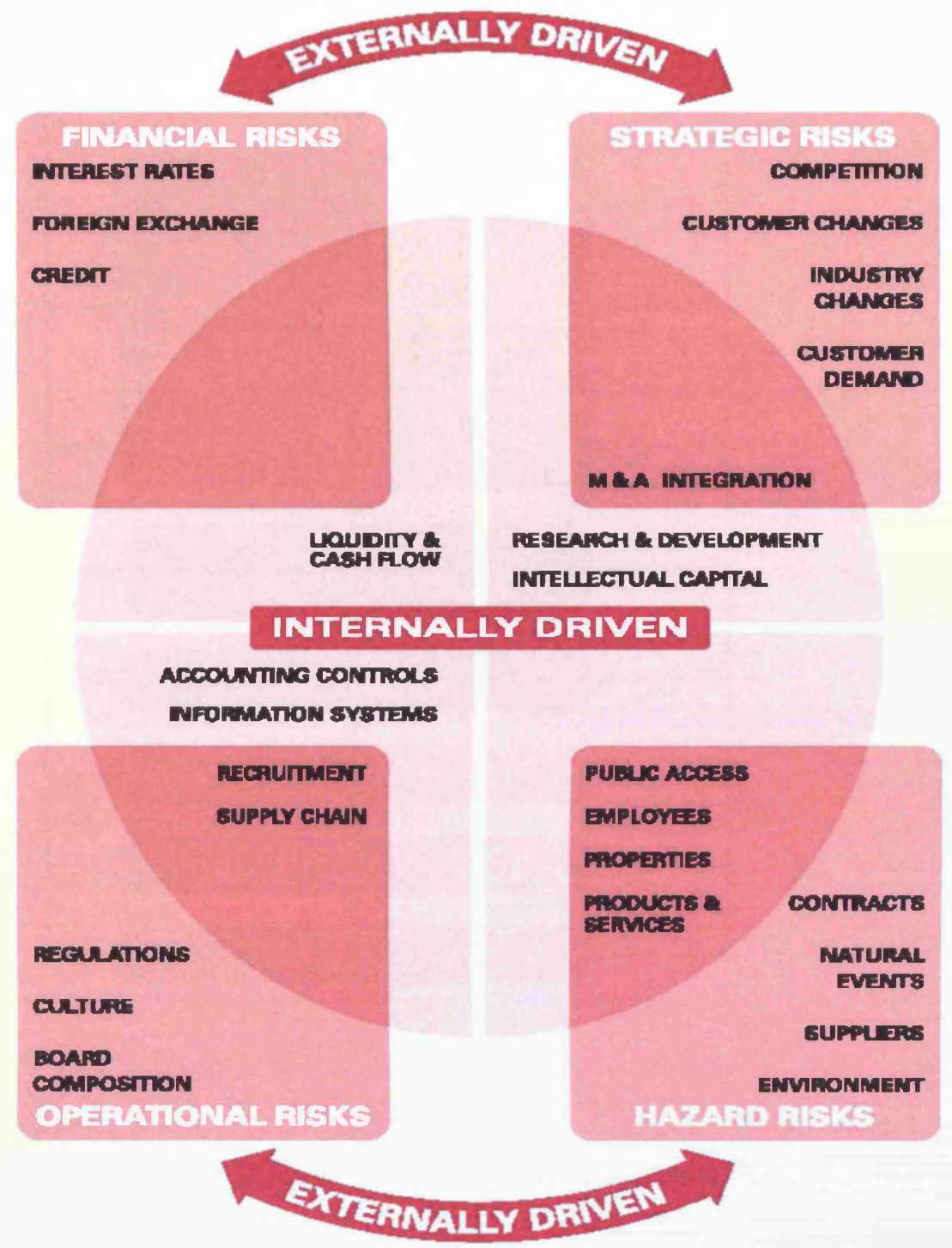

Figure 2-9 - Examples of the Drivers of Key Risks (IRM et al 2002)

The standard shows the drivers for risk, as represented in Figure 2-9, in terms of internal and external factors and further categorises these into types of risk which are financial, strategic, operational and hazards. It uses this picture to provide a Venn diagram of the drivers in relation to their categorisations. 


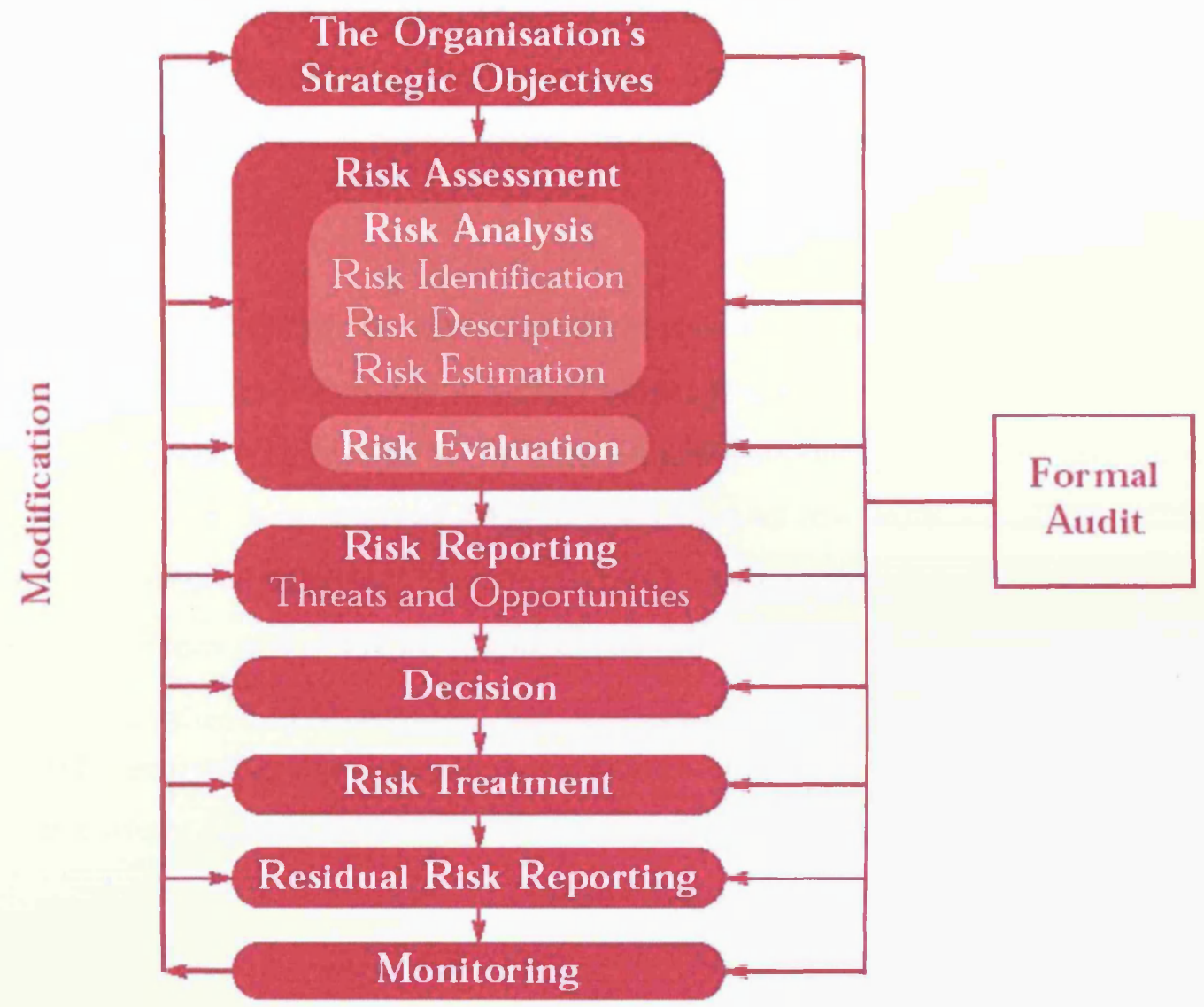

Figure 2-10 - The Risk Management Process (IRM et al 2002)

The standard focuses firstly on risk analysis and evaluation from the ISO guide 73 (2002) definition of risk assessment before discussing the following steps of the process. It does not however return to discuss the first step 'The organisation's strategic objectives'.

The basic process, shown in Figure $2-10$, is again similar to many that have already been mentioned by ICAEW, Boehm etc with the added specification of nested processes. This is seen as a hierarchy of processes - within the risk management process is the risk assessment process, within risk assessment there is risk analysis and risk evaluation, risk analysis in turn has its own set of processes. The standard suggests that risk identification, description or estimation can not be carried out without the con-current execution of the parent processes, running three processes at the same time; this supposition 
is not pragmatic or practical, a risk will be described when it is recognised this may be in conversation or formal review. The rest of the risk management process and all of its sub processes do not need to be executed to carry out this activity.

The standard also defines methods and tools although in the situation where all processes are nested it is not clear where these fit into the overall structure of applying risk management. Another issue with using a nested structure in this way is that it is unclear how to start or end the work - if each process opens up into a plethora of more detailed processes the complexity, cost, resource needs and value of the task could be hidden or lost.

When investigating the detail of this process other issues arise, in many cases the document seems to be unsure which process it is talking about. It refers to risk assessment in a number of places where estimation or evaluation would appear to be the focus of the statement. The sheer number of relationships and transitions which can be followed within the process mean that it becomes meaningless which is compounded by the use of terms including 'Hazard risks' which are difficult to interpret.

Much of the terminology used in the standard is defined in ISO/IEC Guide 73 (2002) which is a positive move to ensure that a shared set of terminology is used within risk management. However it also states in at least one place that it doesn't agree with the terminology in the ISO guide and so uses the term to mean something else.

IRM are proposing the same Risk Management process which companies have been refusing or failing to implement for nearly 20 years and in light of the recent credit crunch has failed again. However this is similar to the information in many national and international standards. CIPS (2007) have taken a more general view recognising some of the issues with risk management best practice, organisational view and scope of management responsibility. Based on these issues they provide a guide to aid in recognising, within their responsibility, what is required of an IT professional 
when assessing and managing risk.

There are three main ways that risk management is considered when it is expressed in a standard. One is to describe the lifecycle of risk management, the second considers risk management as a process and the last is a variation of the two, not really sure if risk management is process or lifecycle based. This section investigates four standards, IEC 61508, ISO 15288, AS/NZS 4360 and ISO 31000, understanding the approach each takes and the benefits and issues associated with the standard.

IEC 61508, which is focused on equipment, does not provide a risk management process or discuss risk management. It focuses on the equipment being used to provide specific functions and considers whether the equipment may cause harm.

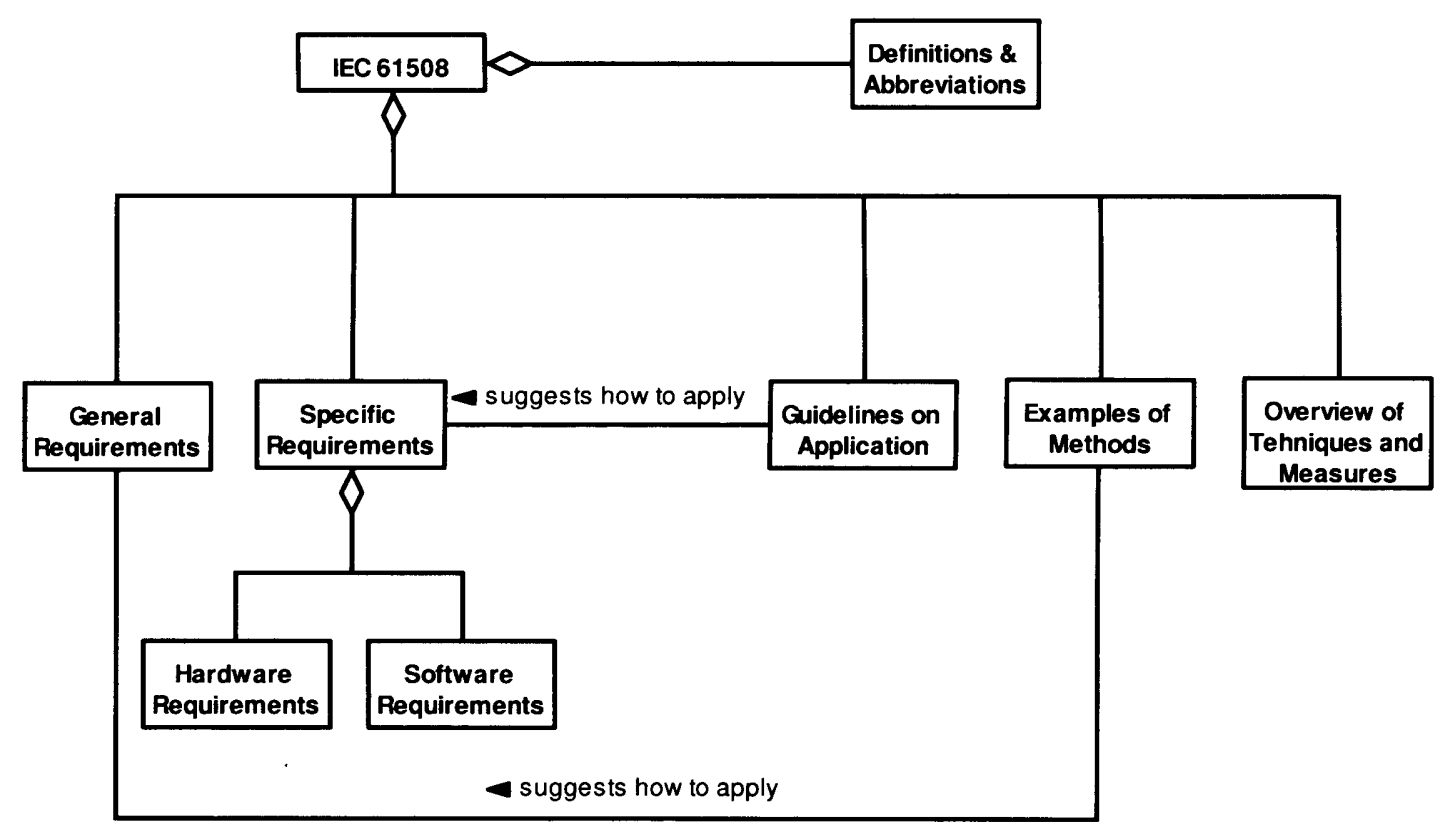

Figure 2-11- IEC 61508 Overview

The focus on equipment can be seen from an overview of the standard shown in Figure 2-11, the requirements in the standard are aimed at software and hardware, more specifically those pieces of hardware or software which provide safety related functions. 
Clause 7.4 of IEC 61508 highlights the need for hazard and risk analysis for which it defines a number of objectives including:

- Determine the hazards and hazardous events of the equipment under control (EUC) and the EUC control system for all reasonably foreseeable circumstances, including fault conditions and misuse.

- Determine the event sequences leading to the hazardous event

- Determine the EUC risks associated with the hazardous event

The standard carries on to define a set of requirements for the hazard and risk analysis ensuring that the hazards and hazardous events are defined, event sequences determined, hazard eliminations considered, likelihood of hazardous events evaluated, potential consequences determined and that the EUC risk shall be determined.

The relationship between hazard, hazardous event and risk can create some interesting reading. It is good to see a clear line between the hazards and risk with the hazardous event being the situation where a person is harmed; risk is the probability of the harm and the severity.

The standard states that 'risk shall be evaluated, or estimated, for each hazardous event. When considering hazardous events and risks together the clear relationship appears to blur as it is known that - in an hazardous event a person is harmed so the probability of occurrence of harm is 1 . The risk from above is the probability of the harm and the severity therefore risk $=1 \mathrm{x}$ severity which could also be expressed as risk = severity of the harm. This statement does not support the added value required of risk management although it is unlikely that the statement is the one the authors intended to make.

The approach the standard takes, only defining the requirements to be fulfilled, leaves an organisation to select and implement its own choice of risk management approaches or indeed to define its own. This can be 
advantageous for those with multiple approaches dependant on project or product, but can make demonstration of compliance complicated.

The standard provides a good overall set of ideas for the consideration of safety related systems, it does not consider risk management but does feed forward into many domain specific standards including EN 50126 (CENELEC 1999), focused on the rail industry, which like IEC 61508 do not define a specific risk management approach or process.

Rather than provide a risk management process they provide a lifecycle which has an element of risk analysis integrated within.

ISO 15288 (2002) is arguably the most widely used systems engineering standard in the world. ISO 15288 was defined provide a set of systems engineering processes, in doing this it attempts to render obsolete a number of existing standards including EIA 632 (EIA 1999), IEEE 1220 (1998) and SECAM (INCOSE 1996) thus removing a some of the complexity of the framework quagmire described by Sheard (1997). ISO 15288 provides processes for systems engineering and a suggested structure in which the processes can be applied. 


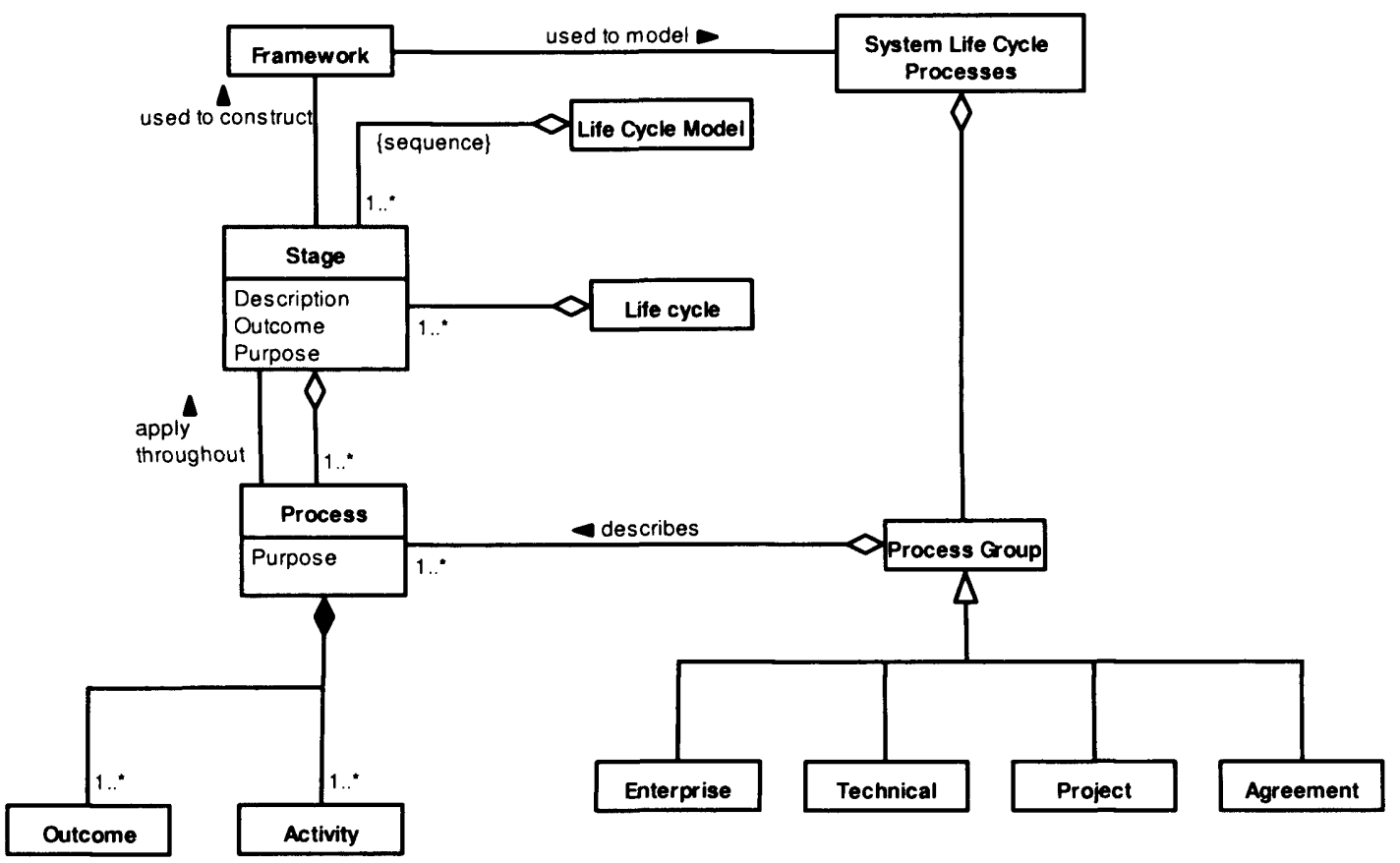

Figure 2-12 - System Life Cycle Stages - Process Structure View

This diagram, Figure 2-12, shows the structure of the System Life Cycle Stages taken from the standard. A framework is used to model the System Life Cycle Process Processes and is constructed from stages made up of a number of processes applied throughout each stage. The life cycle is made up of stages and the life cycle model provides the sequence in which the stages are executed. The System Life Cycle Processes are categorised into 4 Process groups: Enterprise, Technical, Project and Agreement. 


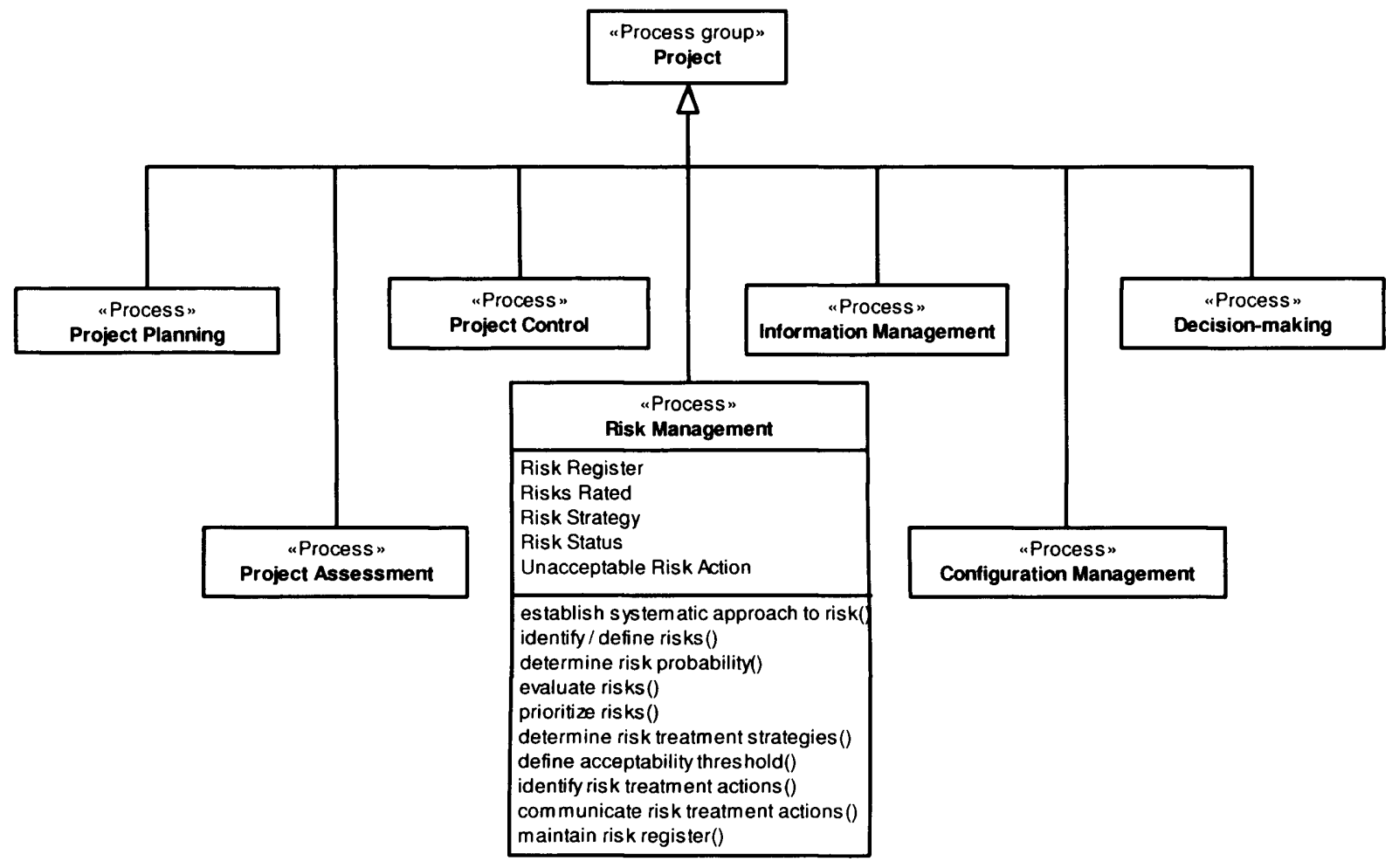

Figure 2-13 - Project - Process Content View

Figure 2-13 shows the project processes of which there are seven: Configuration Management, Decision-making, Information Management, Project Assessment, Project Control, Project Planning and Risk Management, it also provides detail of the risk management process. The detail takes the form of expected outcomes and tasks, these are shown in the two boxes below the process name and are abstracted from the text of the standard.

ISO 15288 is not a risk specific standard. However it still considers risk management a necessary part of a system life cycle and as such defines the outcomes and tasks which should be performed. It does not in any way define a flow for these tasks leaving it to the organisation to tailor as is applicable, as it does with all processes throughout the standard.

The standard provides a very good overall framework for understanding system lifecycles and presents an approach to defining them which enables processes to be re-used throughout the lifecycle rather than used once as some lifecycles would suggest. 
Historically the application of lifecycles has been a topic of great discussion and is presented by Royce (1970) and McConnell (1996). ISO 15288 reflects much of the learning discussed in these papers and is considered the key standard for understanding systems lifecycles by many including the International Council on Systems Engineering (INCOSE) whose systems engineering handbook (2007) is based on the standard. More recent texts focus on the project planning effort (Zwikael and Sadeh 2007) or managing the supply chain ( $\mathrm{Wu}$ and Olson 2008) and (Neiger et al 2009). Although these perspectives provide useful research they do not provide an overall approach to applying the risk management process.

The tasks within the process are presented in the same terminology as the other standards which have already been considered. Two main differences exist; the first is that as no flow has been defined the user is not presented with an excess of bi-directional relationships; secondly there are no oversized steps which connect to every other step here only given tasks which relate to risk e.g. communicate risk treatment actions' are presented. This provides a useful transferable module which can be integrated with other processes.

ISO 15288 does not provide a deep and all encompassing explanation of risk management but it does provide a good overview to work from within a framework which can be applied in most situations and organisations. The risk management process it describes is commensurate with those from other standards and best practice models including those already discussed. This standard is aimed at providing capability for the whole organisation rather than a single risk management focus.

AS/NZS 4360 (2004) is an Australia/New Zealand national standard which is applied internationally including use in Europe on the CORAS project (Lund et al 2004) as risk management process on which the work is based.

The standard provides a set of guidance which is aimed at assisting an organisation in the improvement of its risk management activities. It achieves this by defining terminology, a risk management process, a detailed version of 
the process and providing some thoughts on assessing current practices and planning.

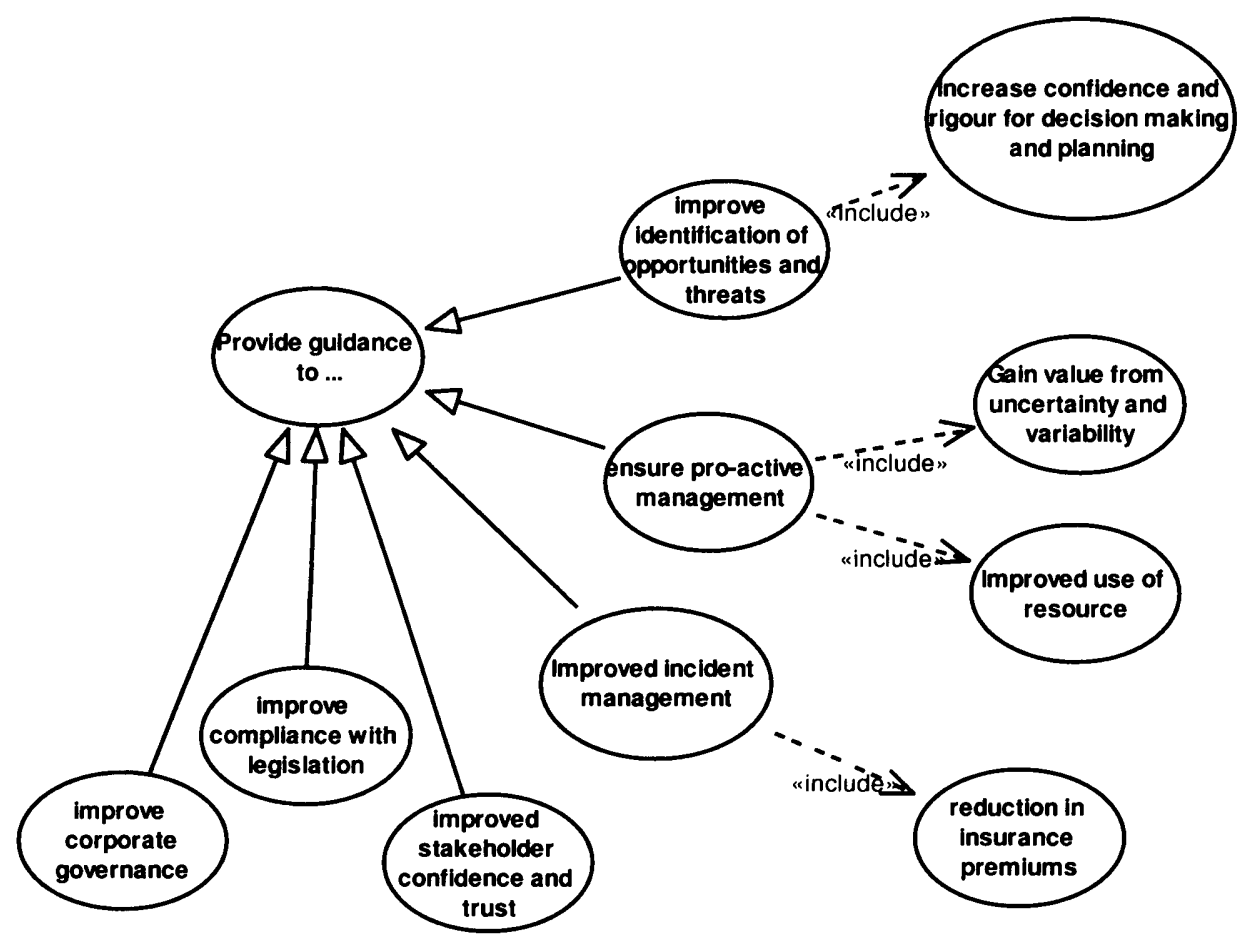

Figure 2-14 - AS/NZS 4360 Purpose

An abstraction of the purpose defined in the standard is shown in Figure 2-14, this shows that the purpose of the standard is to provide a generic set of guidance which is focused on improving identification of opportunities and threats, ensuring pro-active management, improving incident management, improving stakeholder confidence and trust, improving compliance with legislation and improving corporate governance.

Along side this breadth of objectives it is aimed at many different activities, organisations and communities. 


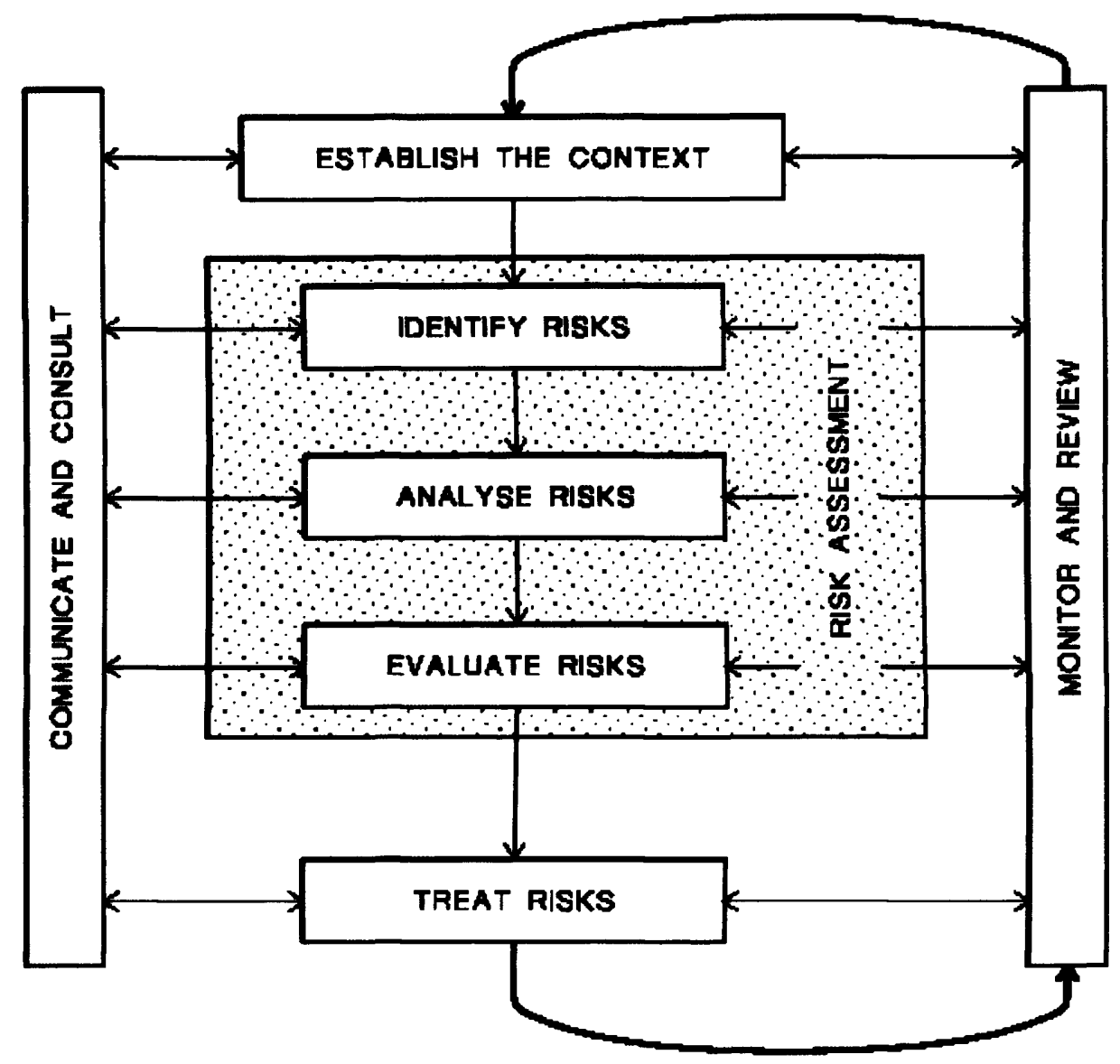

Figure 2-15 - Risk Management Process - Overview (AS/NZS 2004)

Within the standard the risk management process, shown in Figure 2-15, is defined by seven main elements:

1. Communicate and consult - with internal and external stakeholders

2. Establish the context - Internal, external \& risk contexts to be defined. Evaluation criteria and the structure of the analysis also to be defined.

3. Identify risks - Identify where, when, why and how events could prevent, degrade, delay or enhance the achievement of the objectives.

4. Analyse risks - Identify and evaluate controls. Determine consequences and likelihood and hence the level of risk, whilst considering a range of potential consequences.

5. Evaluate risks - Compare levels of risk against the criteria and consider the balance between potential benefits and adverse outcomes.

6. Treat risks - Develop and implement specific strategies and plans for increasing potential benefits and reducing potential costs. 
7. Monitor and review - Monitor the effectiveness of all steps of the risk management process. Risks and the effectiveness of treatment measures need to be monitored to ensure changing circumstances do not alter priorities.

To consider this process in more detail elements 2-6 have been extracted and are shown in Figure 2-16. This diagram shows the theoretical or expected flow through the process.

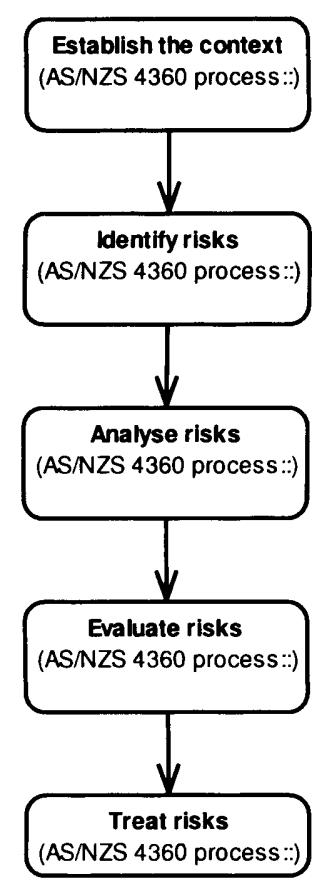

Figure 2-16 - Process - assumed flow

What looks like a simple approach, a flow of five activities, has a number of issues including the lack of decisions, in-ability to re-visit work, no clear start and end (this is currently assumed) and no artefacts.

The lack of artefacts appears to be an issue with the overall approach to the definition of this process. The artefacts which are missing will hold information which it is imperative to value of carrying out the process. The need to record the process is left to an additional statement outside the process.

The overall picture becomes more unclear when reading the text relating to each element, in section 3 of the standard, this talks of steps, activities and 
stages of the risk management process leaving the reader at an overall loss to know how this process is constructed.

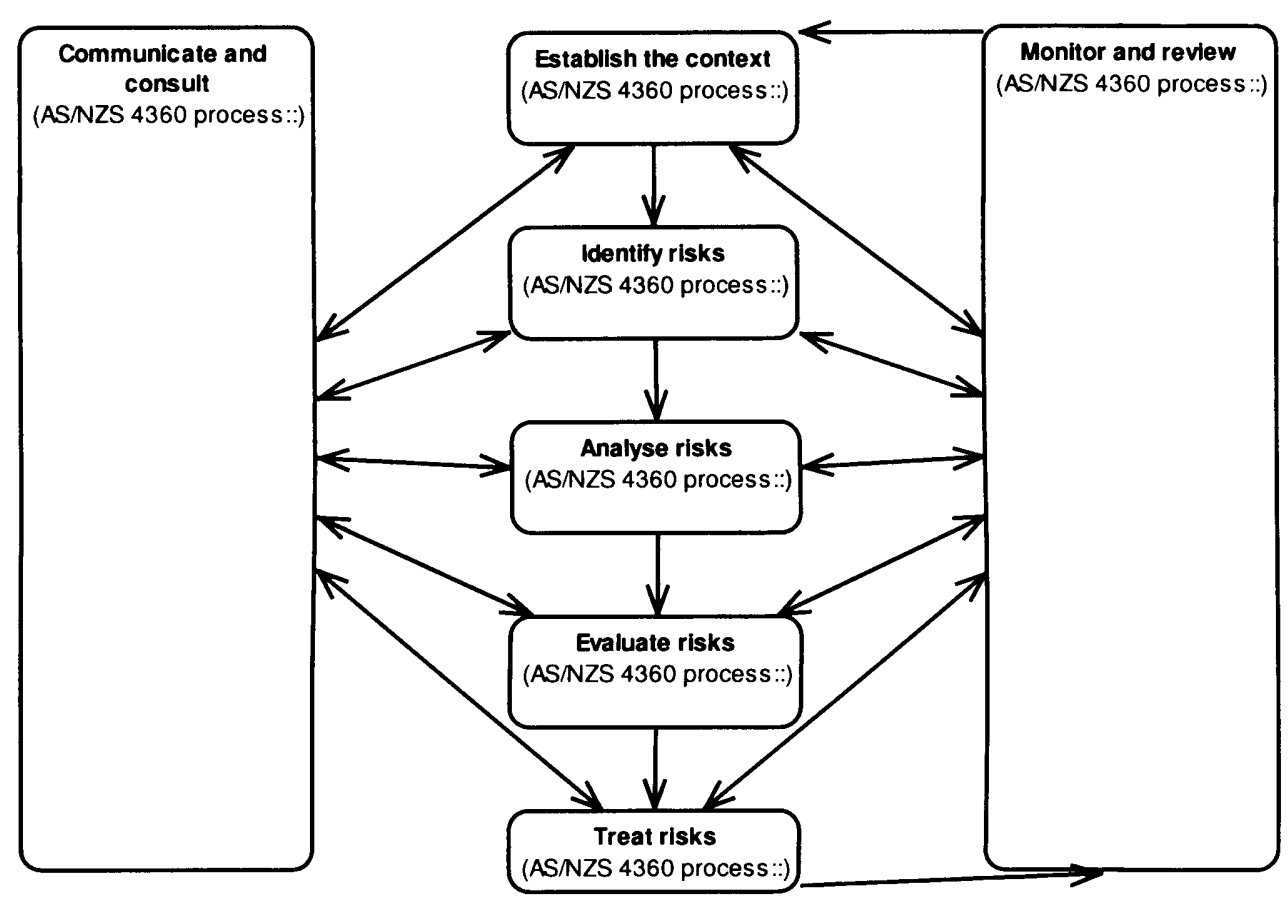

Figure 2-17 - Process - additional flows

This once simple flow has been transformed, through the addition of elements 1 and 7 to the diagram (Figure 2-17), into a set of flows which can not be comprehended let alone calculated. Further to the sheer number of options and paths that can be taken there are also questions as to the meaning of the numerous two way relationships between elements.

The sheer complexity within this process starts to suggest that this is not a process at all but something much larger and more complicated. Although a bad example for integrating activities and information flow the standard does try to remind the reader and sets a good example for integrating with other processes within the organisation.

Twenty seven terms are defined for use within the standards risk management process some of which have cross references to other terms within the list. Each term is accompanied by explanatory text and notes giving some context to the term presented. 
There is a breadth to these terms ranging from 'Event' to 'risk management framework' and 'Stakeholders' giving a grasp of the terms used across risk management and organisations in general. There is also depth to the terms considering 'Control assessment', 'Frequency' and 'Risk sharing'.

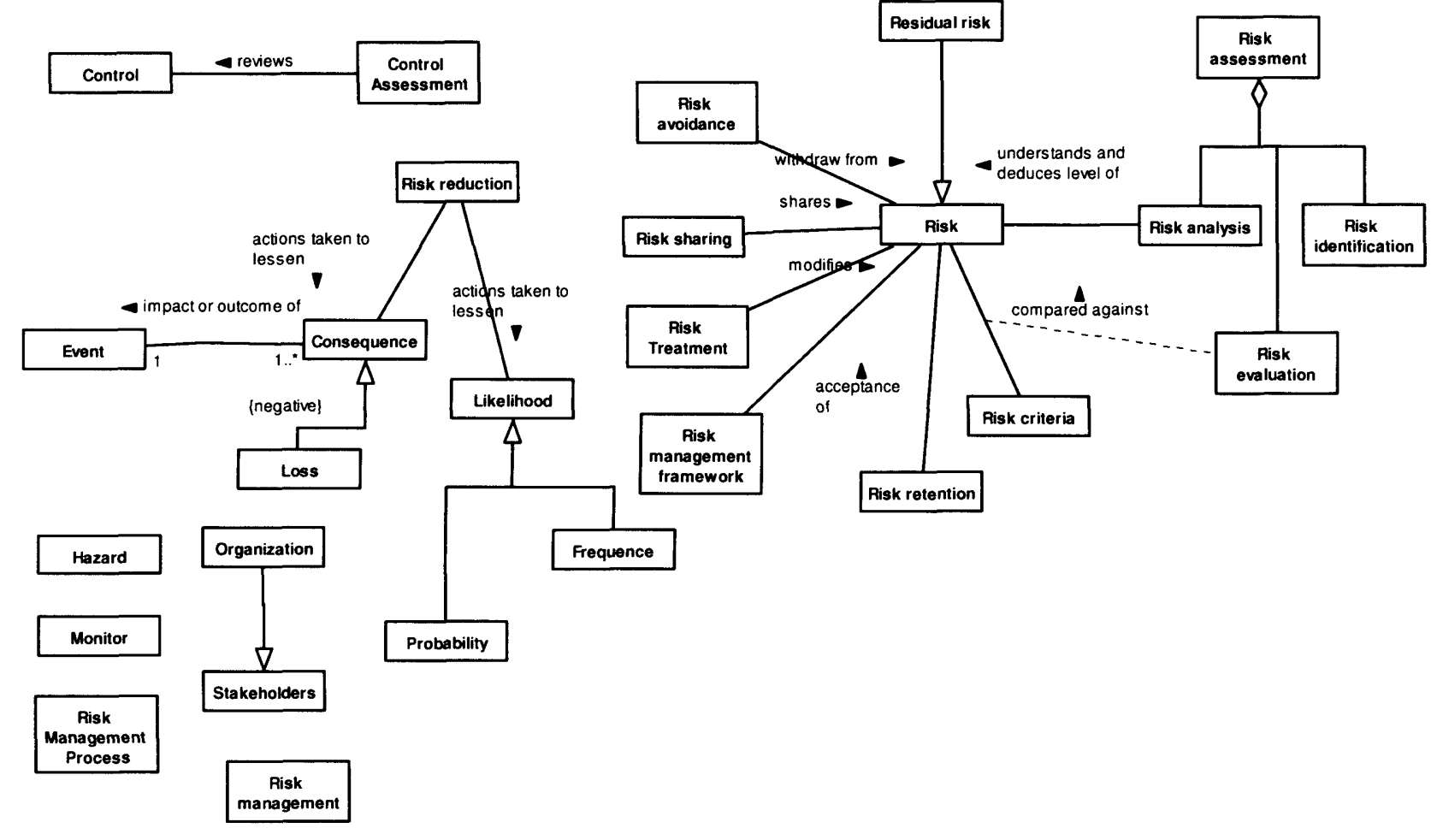

Figure 2-18 - AS/NZS 4360 Terminology Quagmire

Figure 2-18 shows a diagrammatic interpretation of the definitions proposed in AS 4360:2004. It also shows the relationships based on the references given in the text. These definitions and relationships highlight some fundamental issues:

- Some terms are not related to any of the other terminology defined.

- Where they are related it is unclear what the relationship is e.g. risk and risk management framework.

- Unclear relationships also exist e.g. risk avoidance is related to risk although the definition describes a 'risk situation'. It is not clear in this case whether it is the risk or the situation which is being withdrawn from; also risk reduction references risk as being associated with consequence and likelihood but this association is not mentioned in 
risk, consequence or likelihood leaving a confusion as to the nature of the relationship.

- A number of terms which would generally be related to risk have not been, specifically consequence and likelihood - although the 'risk reduction' definition does suggest that there may be a relationship between them.

- Duplication of definition risk reduction and risk treatment either lessen or modify risk, the difference between them seems to be only the level at which they are applied; one to risk the other to likelihood and consequence

Many of these issues could be resolved with a simple review based on the diagram above. It is too often the case that list of terms and definitions are created and published without a full understanding of the relationships.

This standard sets out with a good set of goals, focused at supporting and guiding organisations through risk management, a summary of the standards intentions is shown in Figure 2-19. This is considered as one of the best examples of a risk management standard and is well referenced across the world. 


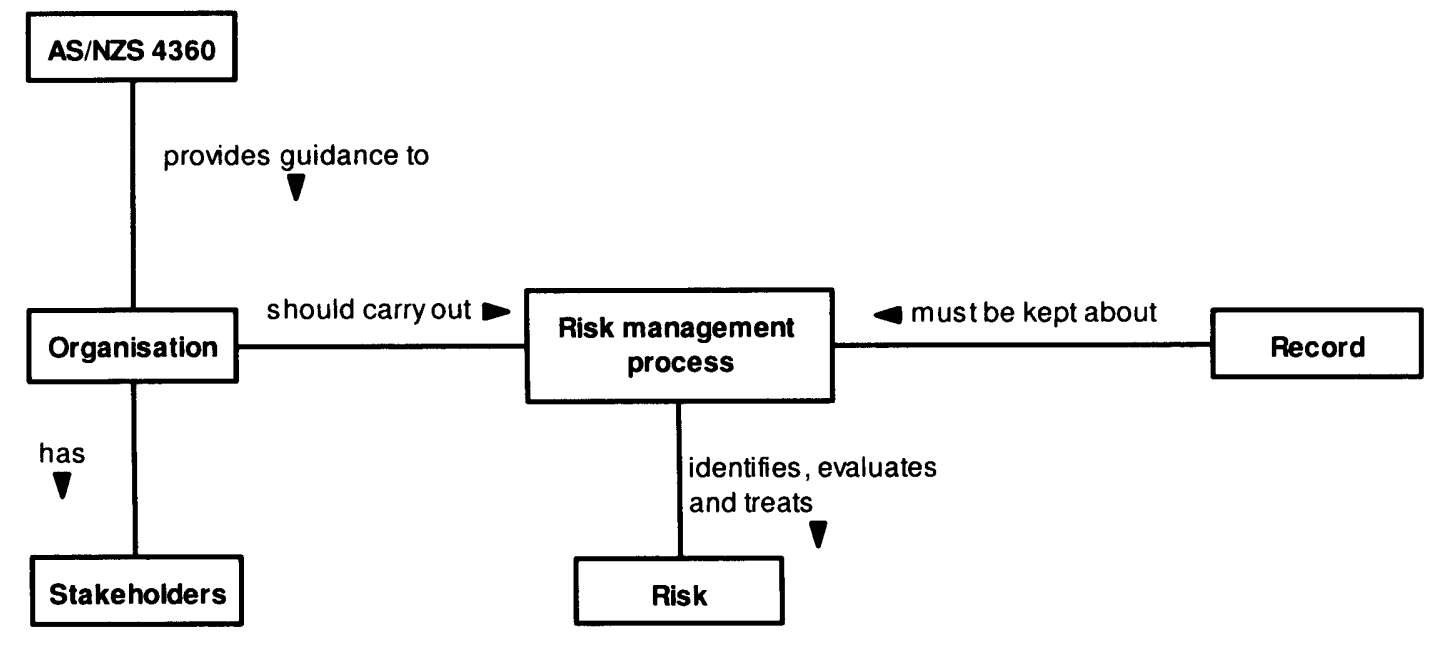

Figure 2-19 - AS/NZS 4360 Summary

Even though this is considered one of the best examples of a risk management standard the detail it presents leaves questions and confusion as to which way the user of the standard should proceed. This coupled with the volume of unconnected terminology can only lead to wildly different interpretations of the standard. The final flaw is the add-on record keeping which reduces the perceived formality and value provided by carrying out risk management.

As a new overarching risk management standard ISO 31000 (2008a) is in a position to clearly define 'Risk Management' its needs and processes. The standard claims to recognise "the variety of the nature, level and complexity of risks and provide generic guidelines on principles and implementation of risk management." and describe the relationship between the principles for managing risk, the risk management framework and the risk management process.

ISO 31000 provides a framework which enables a business level view of risk management to be taken. 


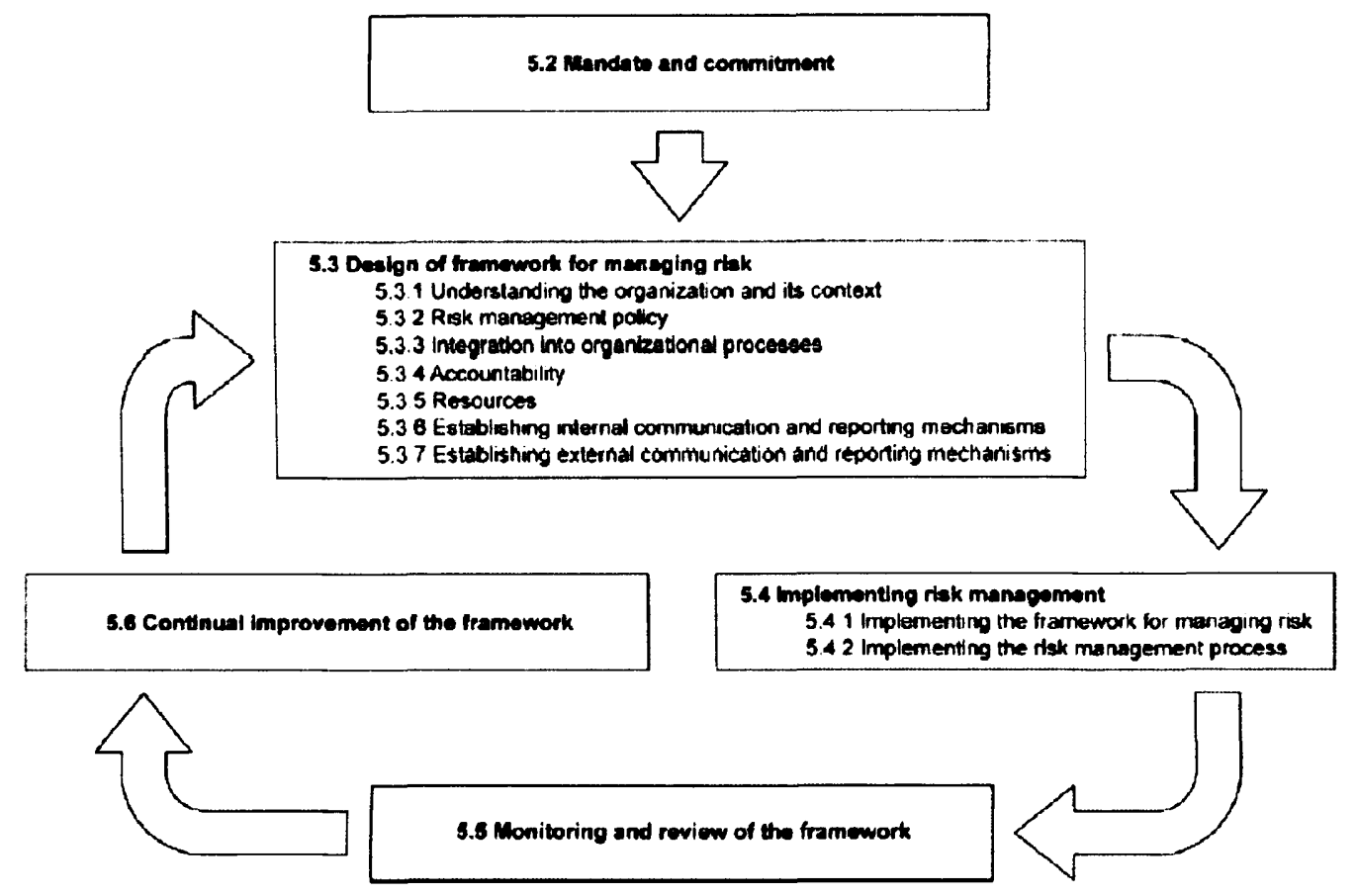

Figure 2-20 - Framework (ISO 2008)

The framework, shown in Figure 2-20, is designed to enable a business to implement the risk management process whilst integrating risk management into its existing management systems. The framework is comprised of five components:

- Mandate and commitment

- Design of framework for managing risk

- Implementing risk management

- Monitoring and review of the framework

- Continual improvement of the framework

Within the implementation of risk management component the standard promotes an iterative approach specifying multiple instances of the risk management process will be required. 


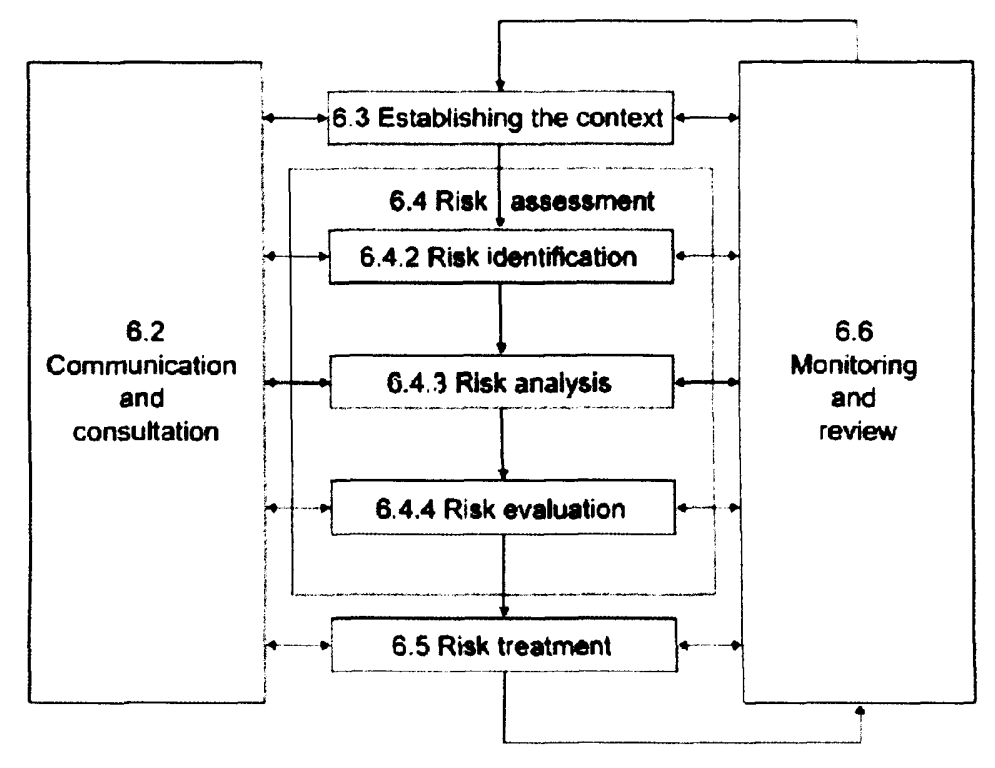

Figure 2-21 - Risk Management Process (ISO 2008)

The risk management process shown in Figure 2-21 has five activities to be carried out:

- communication and consultation,

- establishing the context,

- risk assessment,

- risk treatment and

- monitoring and review

The risk assessment activity is further defined by three sub-activities; risk identification, risk analysis and risk evaluation as defined in the ISO guide 73 (2007).

The Framework defined by the standard adds a level of business integration which has not been observed previously in other standards. Although it is similar in style to the spiral model defined by Beohm (1988) which provides a project level framework for software risk management, the ISO 31000 framework should enable a level of senior management buy-in and action which has not been in evidence previously at an organisational level. 
The specification of multiple instances of the risk management process is an improvement. Many other standards including BS 6079 (BSI 2000) leave the reader to decide whether to use the process once per risk, once per project or once per organisation. The standard also specifies that risk identification is only carried out once for each instance of the process which leaves risks identified later in a project or execution without the ability to be considered or analysed.

The standard suggests that whenever a risk assessment is carried out risk identification, analysis and evaluation will also be carried out. This may be an issue with the terminology used in the standard, which is based on ISO Guide 73 (2007) to be discussed in more detail later. In many cases risk identification, analysis and evaluation have to be carried out separately due to issues like volume of information and time.

Overall the Risk Management process specified is very similar to AS/NZS 4360 and exhibits the same advantages and disadvantages, mainly the inability to follow a process flow.

Although still in draft form this standard does not currently exhibit the clear concise views which have been missing from the risk management domain and are required to direct risk management across industries in this global society. The use of guide 73 for the terminology provides a single reference point for risk terminology although the clarity of the terms and their relationships needs to be investigated further. 


\subsection{Terminology}

Issues with terminology have already been highlighted along with the importance of having a consistent set of terminology on which to base practices. This section will investigate literature which defines general risk terminology focusing on guide 73 a key reference. The section will also focus on the definition of a risk which itself provides much confusion.

The ISO Guide 73 Risk management - vocabulary provides the basic definitions of risk management generic terms. The aims of the guide are to "encourage a mutual and consistent understanding, a coherent approach to the description of activities relating to the management of risk, and use of risk management terminology in processes and frameworks dealing with the management of risk."

The guide is split into four groups of terms:

- Basic Terms

- Terms related to people or organisation affected by risk

- Terms related to risk assessment

- Terms related to risk treatment and control

This work focuses on the basic terms referencing specific relationships where relevant. 


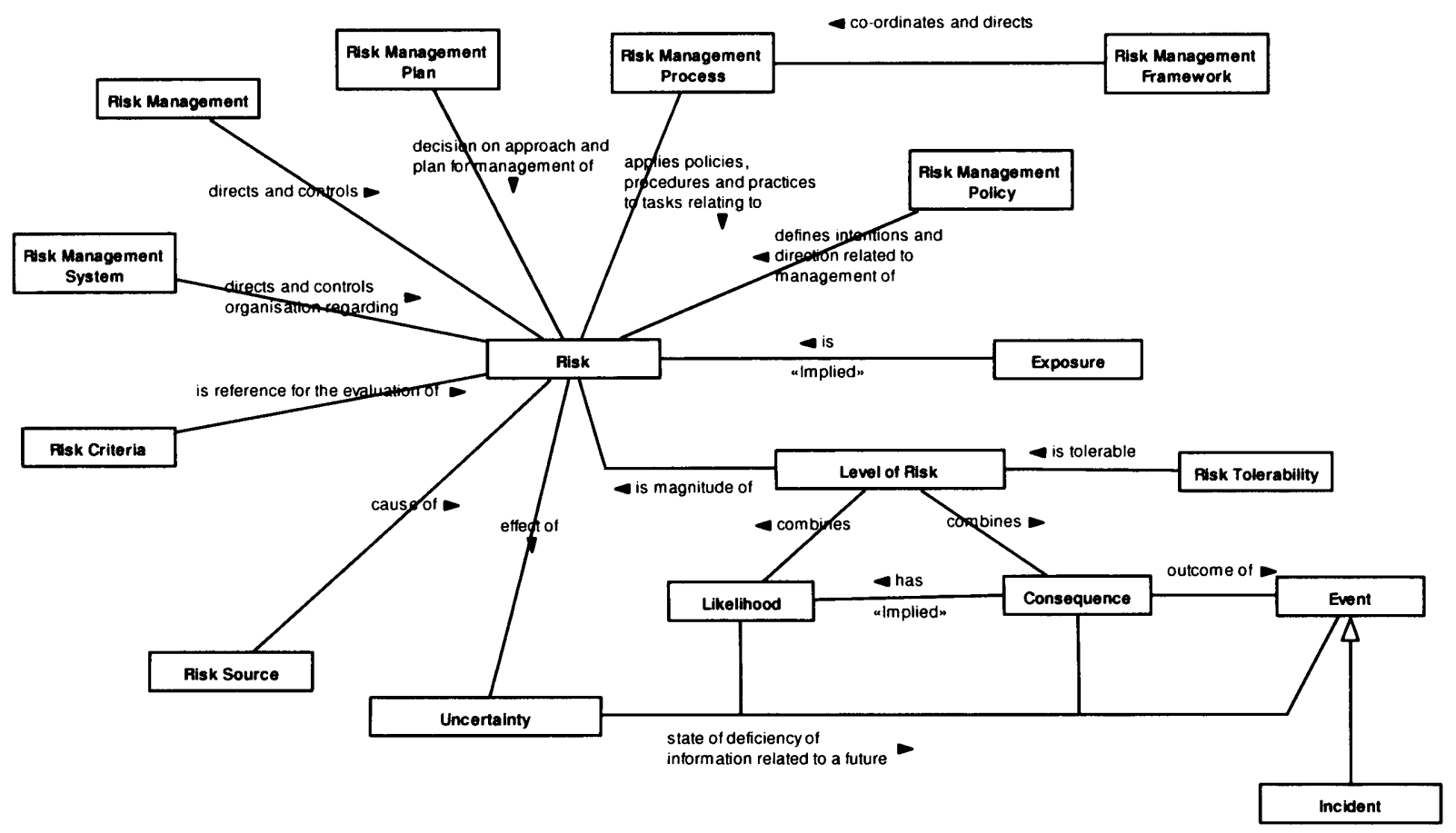

Figure 2-22 - Risk management vocabulary

The guide defines 17 basic terms many of which have multiple definitions including risk which has one main definition and two variations. Each definition provides references to other defined terms used within.

The diagram in Figure 2-22 shows the terms and their relationships as defined by the guide and has been defined in this way to enable consideration of the terms and relationships defined within the standards. It will also be used to enable a comparison to be carried out between the terminology within guide 73 and AS/NZS 4360. The figure shows that more than half of the terms defined related directly to the definition of risk. The number of direct relationships to the definition of risk presents issues when factoring in the general lack of relationship from these terms to any other definition. The main issue is that many of the terms appear only to be related to the definition of risk as they include the word risk. This concern is supported by the repetition of relationships, two relationships 'direct' and 'control' risk, three relationships provide intentions, policies, procedures or decisions relating to the management of risk.

Within Figure 2-22 there are also two relationships marked as <<implied>> these are not stated by reference but is suggested by the notes associated 
with the definition.

When the same modelling principle is applied to the other sets of terminology similar results are achieved, risk treatment for example is referred to as a process, activity and a measure (solution) in ISO 16085 (2006)

On the whole the definitions are unimaginative and unclear for instance "Risk Management System - management system to direct and control and organization with regard to risk 3.1.1". The re-use of the defined term in the definition only leaves six words to define the term. This only reference is to Risk, not to the risk management process, plan or policy which the notes suggest.

The Australian standard AS/NZS 4360:2004 provides its own set of terminology, Figure 2-23, which has been discussed earlier in this chapter. The issues discussed will be used to provide a comparison for those in the ISO Guide 73.

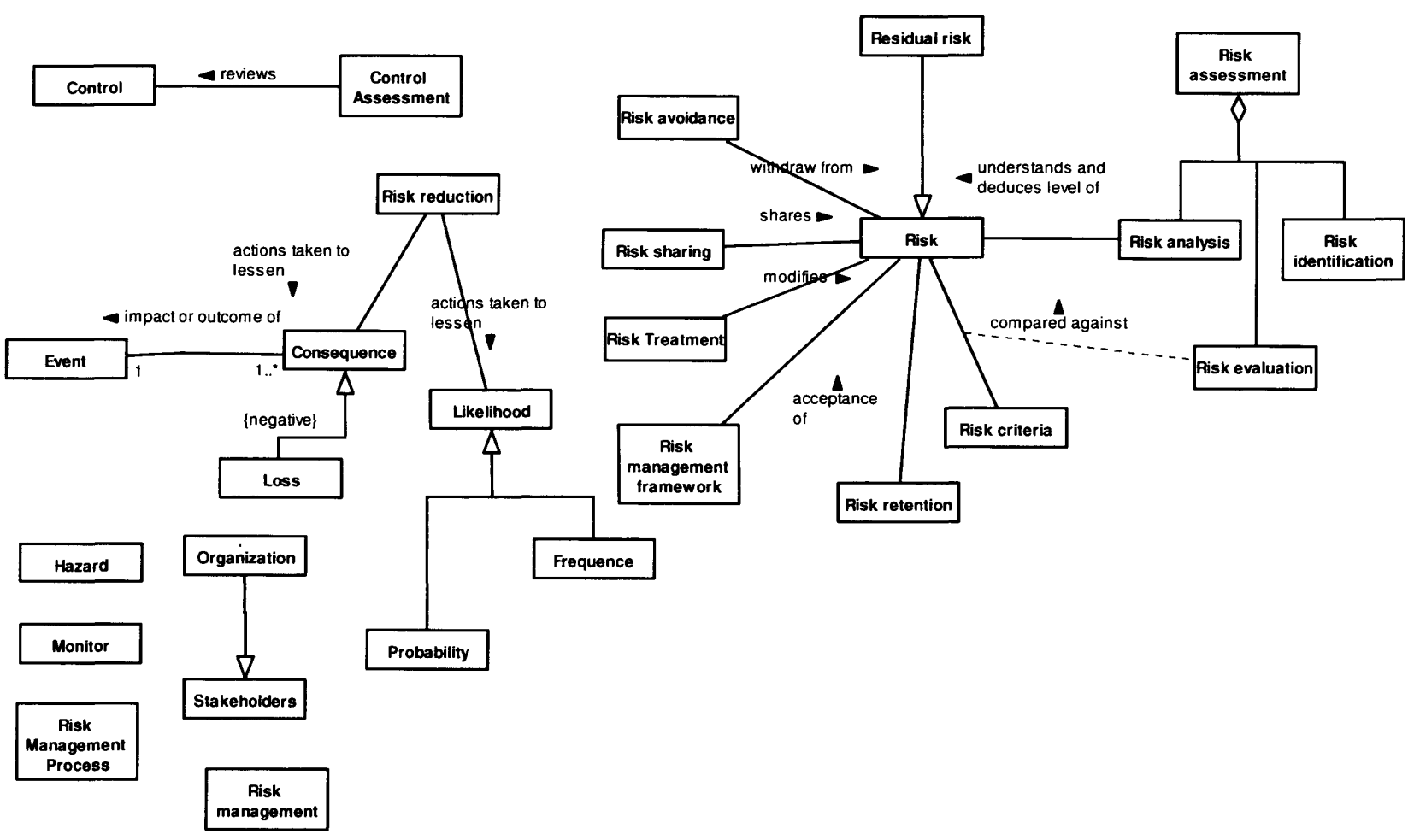

Figure 2-23 - AS/NZS 4360 
Many of the issues are exhibited by both standards.

- Much of the terminology being related directly and only to risk rather than other relevant terminology from the set.

- The lack of direct relationship between risk and consequence and likelihood as in both standards it is implied that there is a direct relationship but this is not clear within the definitions.

Both standards have their own advantages.

- ISO 31000 provides relationships from all of its terms which is a step forward from AS/NZS 4360

- AS/NZS 4360 incorporates process terminology where ISO 31000 separates it into a different set of definitions.

There are other similarities which may be useful to note.

- The relationships between consequence and event are almost identical

- They both provide detail regarding the composition of risk

- They both ignore the composition of most terms other than risk.

The fact that both standards have similar issues with the definition of risk and the direct relationships they try to assert between management terms and risk highlight the need for a formalised approach to the definition of domain terminology.

The Unified Modelling Language (UML) Profile for Modelling Quality of Service and Fault Tolerance Characteristics and Mechanisms Specification (OMG 2008b) provides a UML profile based on the AS/NZS 4360 (1999) terminology, within this profile a risk is defined as being made up of a frequency and a consequence. This definition is shown in a note and in the 2004 version of the standard. 


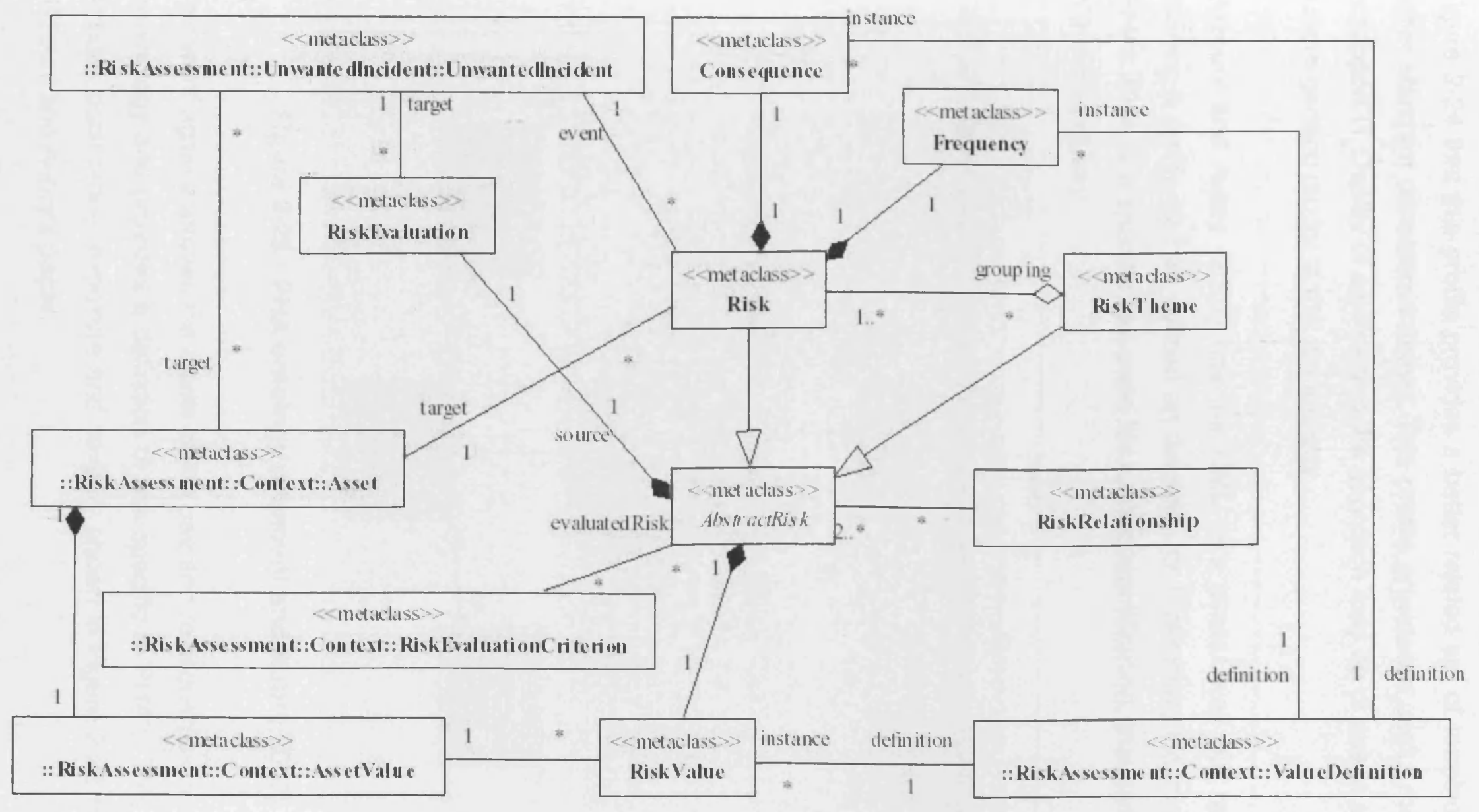

Figure 2-24 - QoS UML profile (OMG 2008b) 
It can be seen by the clarity and volume of relationships between terms in Figure 2-24 that this profile provides a better related set of terminology than either standard considered above. This profile provides a useful meta model to support IT Quality of service and the approach may be of use in supporting a more generic model of risk terminology.

Mazouni and Aubry (2007) use the UML in a similar way but rather than defining a profile he has defined an ontology for Preliminary Hazard Analysis (PHA). PHA is a specific tool used for hazard identification, this case applied in the rail industry.

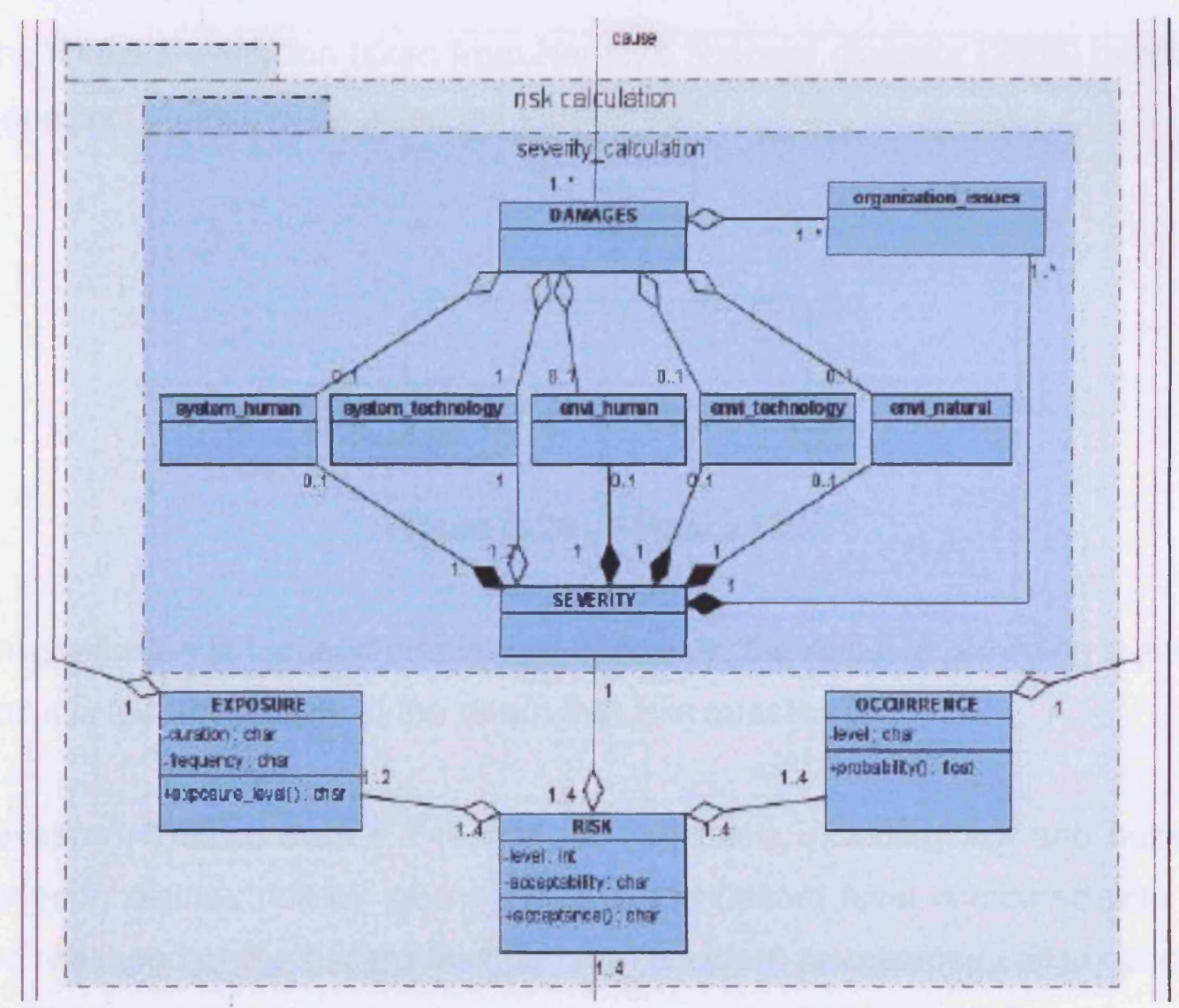

Figure 2-25 - PHA ontology (Mazouni and Aubry 2007)

This work again improves the clarity of the use and relationships between risk terminology and provides a definition of risk specific to PHA's. This definition includes occurrence, exposure and severity shown in Figure 2-25 taken from Mazouni and Aubry's paper. 


\subsubsection{Risk Definitions}

The numerous definitions of risk and its surrounding terminology along side the lack of consistency in the relationships between terms provide a large and complex issue. To further understand the root cause of this issue focus will be placed on the on the central terminology 'Risk' to which authors and standards seem more able to relate detail.

This work will consider a number of risk definitions from texts and standards prior to drawing conclusions regarding overall issues with the definition of risk.

The financial definition taken from Harvey's financial glossary (2008) provides a succinct definition, depicted in Figure 2-26.

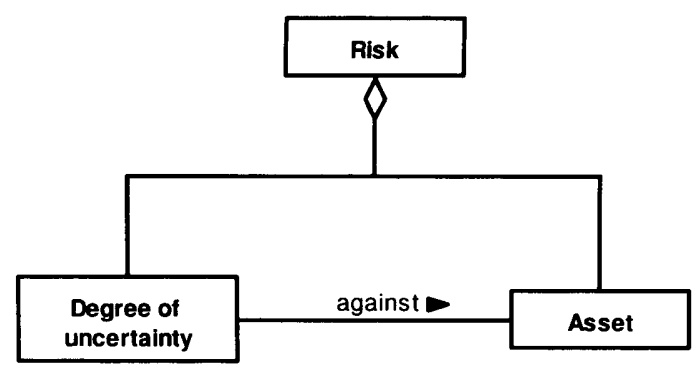

Figure 2-26 - Finance risk

This definition is focused on the loss of money, the Asset is providing a return and it is the uncertainty of the return that is in questions.

Leveson (1995) provides a number of definitions including risk and hazard. Leveson defines risk by saying, "Risk is the hazard level combined with (1) the likelihood of the hazard leading to an accident (sometimes called danger) and (2) hazard exposure or duration (sometimes called latency)." 


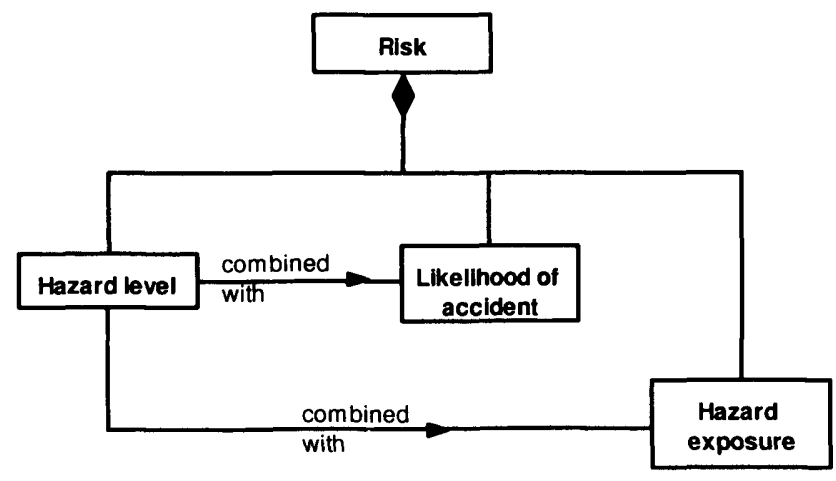

Figure 2-27 - Leveson risk

"Hazard exposure" requires an understanding of how long something will be exposed.

Although when in time an event may occur cannot be told, it could be after five minutes or one year. The point is that the same outcome could occur but with this definition it appears that the risk will be different. The point Leveson is trying to make is that the longer you are in a hazardous state, the more likely an accident is to occur e.g. the longer you sit in a tree the more likely you are to fall out.

Roland and Moriarty (1990) states "risk is associated with likelihood or possibility of harm. Put another way, it is the expected value of loss."

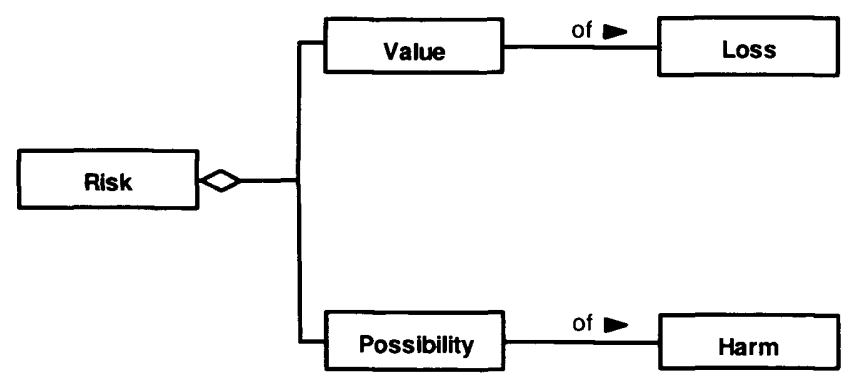

Figure 2-28 - Roland and Moriarty's risk

"Risk" may still exist if either "Possibility" or "Value" is not present; the empty diamond denotes this. This definition shows two very different ideas of what risk is, although Roland and Moriarty have used one as an example of another. They are saying that the "Value" of "Loss" is equivalent to "Possibility" of "Harm". 
Storey (1996) in his book on safety critical computer systems also defines both risk and hazard. Storey poses a definition which is that "risk is a combination of the frequency or probability of a specified hazardous event, and its consequence."

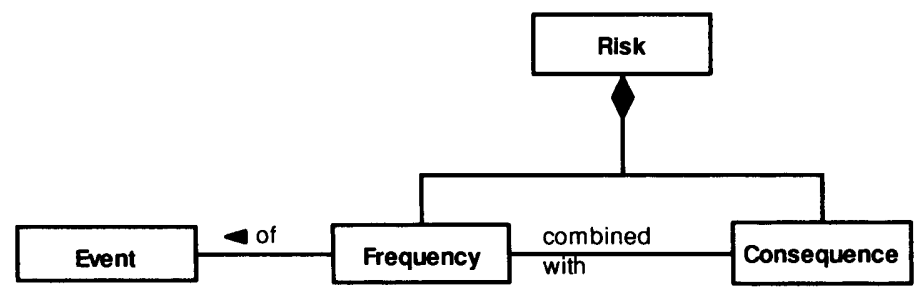

Figure 2-29 - Storey risk

It is the "Frequency" of an "Event" combined with the "Consequence" that makes up "Risk". There is a problem, which can be seen in this diagram, the use of the word 'combination'. The problem here is that there is no explanation of how to combine the relevant information. It is unclear as to whether the subjects are added, subtracted, multiplied or combined by another means.

BS 6079 (BSI 2000) describes risk as the 'uncertainty inherent in plans and the possibility of something happening that can affect the prospects of achieving business or project goals'.

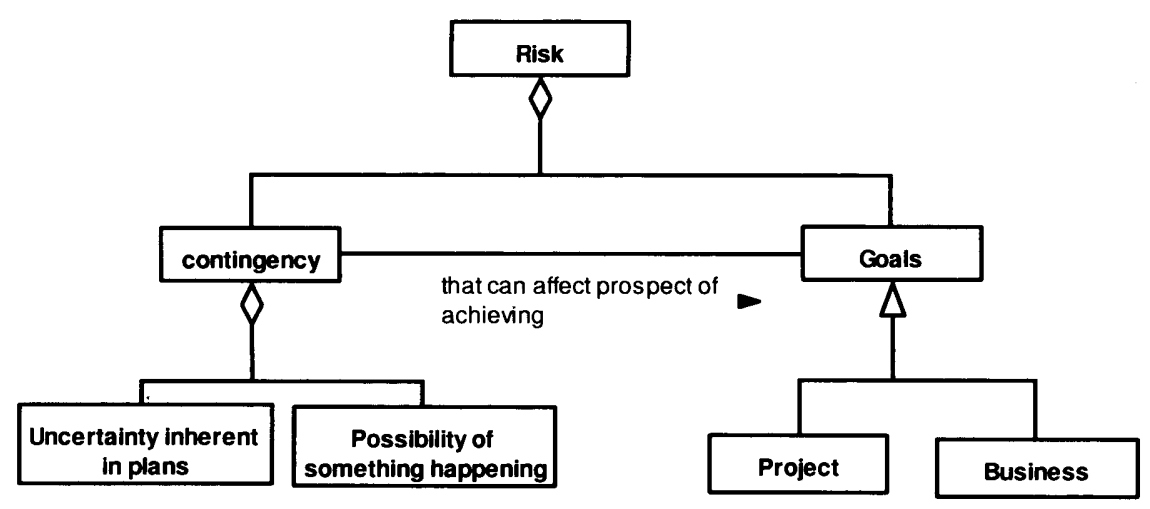

Figure 2-30 - BS 6079 risk definition

This definition uses vague terminology - it is not difficult to agree that there is 'the possibility of something happening'. This definition is bordering on the possibilistic discussed by Clarke (2007). 
BS 8444 (BSI 1996) defines risk as the 'combination of the frequency, or probability, of occurrence and the consequence of a specified hazardous event'.

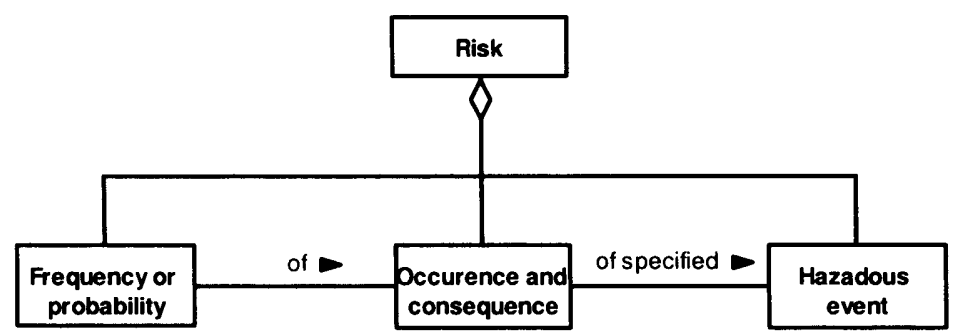

Figure 2-31 - BS 8444 risk

An interpretation of the definition in BS 8444, shown in Figure 2-31, provides an understanding of a strong relationship between 'hazardous event' and 'occurrence and consequence'. it is strange however that Occurrence and consequence are combined as there could be many possible consequences for any one occurrence.

EN 50126 (CENELEC 1999) defines risk as 'the probable rate of occurrence of a hazard causing harm and the degree of severity of the harm'.

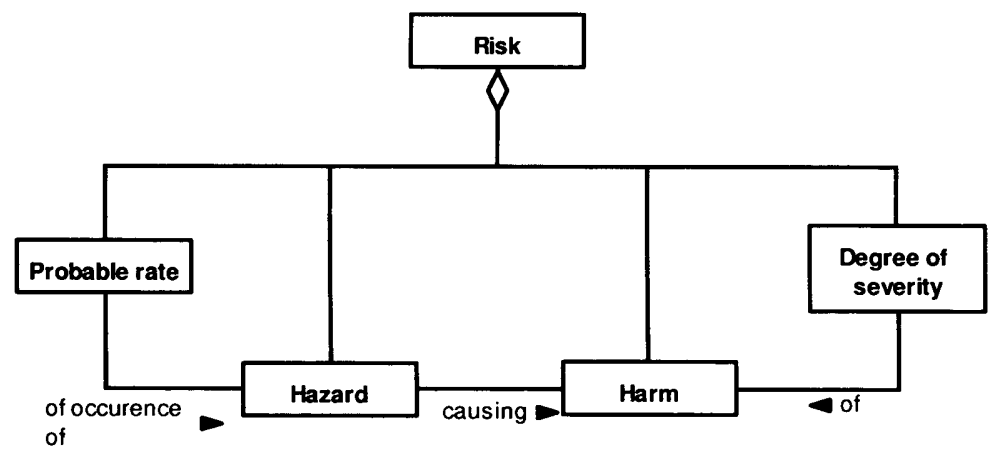

Figure 2-32 - EN 50126 risk Definition

Figure 2-32 shows a level of separation between the probability (Probable rate) and the harm. This separation raises questions as to which probability is specified in the definition. It is not clear whether it is the probability of the Hazard occurring or the harm. 
Seven definitions of risk have been considered and a number of similarities can be identified including; use of probability, focus on outcomes, use of hazard, dependency on timing and multiple interpretations.

The first of these similarities the use of probability can be seen in many of the definitions through the use of words like frequency, likelihood and uncertainty. These terms all infer the use of probability in risk. For probability to mean something in terms of risk it must be relevant to its mathematical definition and therefore the sum of all probabilities must equal one.

The definitions above all in some way refer to an outcome. Some consider this to be harm, accident or consequence. The problem with accident and harm is that they only take into account one view of the argument - the negative effect. To gain a fuller picture future (Hollnagel 2008) and positive (Flage and Aven 2009) consequences must also be considered.

A number of the definitions incorporate Hazard, a word which could be considered to have as many definitions as risk. In general it is used to signify an event pre-ceding the outcome or consequence under scrutiny (Woodruff 2005, Stevens and Thevaranjan 2009 and Gamper and Turcanu 2009).

The use of words like consequence and hazard ensure that a reader of the definitions will be considering issues beyond or before the 'event' that is the focus as suggested by Woodruff (2005). Considering the chance of an individual being rushed to hospital, as a consequence, has very little to do with the risk that this is the consequence of.

The last point, interpretation, is amplified by Roland and Moriarty (1990) as they provide two definitions within one. Their definition discusses possibility and harm and talks about Value and loss as an example. This implies that value would relate to possibility and loss to harm. Although this sounds reasonable the value of loss, say $£ 5,000$ by itself cannot be linked to any probability. This may be a bad example on their part but it goes to show risk is easily misunderstood. 
Remenyi and Heafield (1996) summaries this discussion well by stating that "Risk is a challenging concept to define, understand and ultimately to manage. This is primarily because risk often means different things to different people." This is supported by Aven and Kristensen (2005) through the statement of their view "that the concept of risk, risk assessment and risk management have not yet been sufficiently developed".

There are however a number of commonalities between the ideas discussed above; one of these is that to understand risk a value must be provided (whether numerical or descriptive) detailing the chance of occurrence of an outcome. Another is that there must be a set of outcomes in order to understand fully the whole set of risk. There will always be events that occur prior to and soon after the outcome that is of concern. Finally, every outcome must be traceable from its cause or hazard.

\subsubsection{Terminology Summary}

In summary there are a number of issues with the term risk and the terminology which is associated with it. Initially there is a lack of coherence between authors and standards including those yet to be published. Those authors or standards providing definitions of more than one term very rarely enable a user to navigate through the terms and the inclusion of timing in the definition of risk, through the use of hazard and consequence, reduces the focus on identifying and defining a risk before it is analysed.

A solution too many of these issues would be to provide an ontology for risk defining the terminology and the relationships between those terms defined. This would enable users to understand the scope of each term individually and provide the associated terms which they would expect to hear in relation to those initially defined.

Each set of terminology along with each industry has its own tools and techniques which will also be impacted by this terminology and would benefit from a single unified source. 


\subsection{Tools and techniques}

Much of the focus of risk management research past and present is on tools and techniques; tools are the software, hardware and document templates in and through which data is captured to give meaning and/or understanding to a risk or hazard. Techniques are the approaches or methodologies used to gather the data and calculate the results for specific risks. Tools and techniques are often grouped together as it can be difficult to differentiate one from the other.

Many types of tool and technique exist to support the definition of a risk, understand causes of risks and to understand consequences. Leveson (1995), The IRM and AIRMIC's risk management standard (2002) and ISO 31010 (2008b) all provide list of tools and techniques. Leveson provides a critical analysis of each whilst ISO 31010 provides a matrix showing in which areas of risk each tool or technique can be applied.

These three references provide a list of 73 tools, techniques or methods. The IRM categorise their list into risk Identification techniques and risk analysis methods and techniques. These categories equate to the areas incorporated in risk assessment according to ISO guide 73 (2002). The latter of the two, risk analysis methods and techniques, groups the techniques into upside risk, both and downside risk.

ISO 31010 states that four of the methods identified apply to all steps in the risk management process these methods are Failure, Modes, Effects and Criticality Analysis (FMECA), Reliability centred maintenance, Structured What If (SWIFT) and Environmental risk assessment. It is more likely that these tools provide information related to terminology which is used in each step rather than as the standard implies - if you have used this method risk management has been carried out satisfactorily.

Many of the tools, techniques or methods provided by the references above have been tailored for specific applications or industries including 
- human factors Cacciabue (2005) and human health Davis et al (2008)

- political acceptance criteria Ale (2005) and local empowerment Nilsen (2008)

- supply chain disruptions Adhitya et al. (2007), procurement Aggarwal and Ganeshan (2007) and operational risk (Dalla Valle and Giudici 2008)

- International project risk management Han et al. (2008) and post project learning Dikmen et al (2008)

- Pedestrian surface evaluation Hunt-Sturman and Jackson (2009)and water treatment Hess and Bernard (2008)

In many cases such as with Kirchsteiger (2008) the use of a tool or methodology is referred to as 'Risk assessment'.

In truth 'Risk assessments' provide a variable output dependant on the user. This is discussed in detail by Leveson (1995) when concluding her tools and techniques discussion. In this discussion she explores results from independent groups applying the same methods to a system and finds inconsistent results with too much variation to be the tool alone.

Tools, methods and techniques provide detailed information regarding a risk or the approach to calculating risk. Many authors are trying to further refine these risk assessments for specific industries and applications. In many cases they are re-defining the terminology of risk along side their tailoring of the risk assessment. 


\subsection{Conclusions}

A number of issues with the existing process and terminology associated with risk management have been identified during the investigation the purpose of which was to review and understand the terminology and processes related to risk management.

The lack of change in the risk management process is a major issue and can be seen throughout this chapter from Bohems definition to the yet unpublished ISO 31000 standard. The lack of change shows that there is little or no recognition that the risk management process may not be fulfilling its aims. The lack of any updates to the process and the inability of the process to fulfil its aims suggest a re-evaluation using a pragmatic approach is required. To ensure that the relevant level of rigour is also incorporated a formal approach incorporating the definition of terminology and artefacts would also be advantageous.

The lack of clear well related definitions hinder progress in risk management. There does not appear to be any recognition that in this semantic world/age loose relationships between terminology do not provide enough definition or scope to the terminology in question. Both authors and standards must recognise that definitions by themselves are no longer enough; ontologies must be defined and published to show relationships between terms as well as their definition.

Due to the variation in scope and depth of application no further work on tools and techniques is proposed. The issues with risk management process and terminology suggest that it is the wider, system view which needs improvement and formalisation. Chapter three will investigate an approach to providing the formalised view of risk management required. 


\section{Research Methodology}

\subsection{Introduction}

This chapter outlines the philosophical and practical approach taken toward the development and implementation of this work. It discusses the appropriateness of phenomenological and positivist perspectives discussed by Remenyi (1998) and selects a research strategy before identifying tactics which may be used for the identification of solutions and the demonstration of the application of the solution proposed. It also considers the implications of generalisation and validation.

\subsection{Methodology}

\subsubsection{Strategy}

It is envisaged that the initial research question will be developed through the understanding of literature providing a document based approach to the initial questions. These questions will be tested through the interpretation of a number of cases. The intention is to take a cross sectional view of these cases providing a variety of contexts in which issues may be identified.

In this way the research will be of an empirical nature understanding concepts based on the experience of the researcher and the processes identified through the initial studies.

It is anticipated that one of the issues within the research area is the lack of ability to recognise and integrate the positivist and phenomenological mind sets, with many people unable to release their positivist scientific backgrounds. With this in mind the intention is to ensure that this work takes a phenomenological approach enabling the identification of those areas which are currently lacking.

Although a mainly phenomenological approach is suggested it is likely that the final study although phenomenological in outlook and application will be 
positivist in nature - the intention will be to search for cases in which the proposed solution does not work.

\subsubsection{Tactics}

Specific research tactics will be applied within the initial evidence gathering case studies and the final application study. The tactics are likely to include:

- Case study

- Action research

- Ethnography

- Participant observer

It is envisaged that a number of issues may arise during the execution of this work due to the implementation of these tactics. Issues include the inevitable bias due to the researcher being part of the team within the case studies and the cultural acceptance of a phenomenological approach within positivist domains.

Where issues with the mind set of participants is an issue case studies may be excluded or discontinued due to the resource and time available to change the organisational culture. Participant observation and researcher in the team issues will be unavoidable as it will be necessary to transfer the knowledge of the proposed solution to other participants, in some cases the participants may not know that research is being carried out, in these cases there will be a long term ethnographic type relationship with the organisations in question.

\subsubsection{Generalisability}

It is anticipated that this work will provide a generalised approach to the management of risk from a phenomenological viewpoint. Generalisation of evidence based on a phenomenological approach is not usually recommended however in this case the work will be carried out in relation to the already generalised definitions of risk management. These existing general definitions will enable direct comparison between the options proposed within this work and current understandings. 


\section{Application}

\subsection{Introduction}

The purpose of this chapter is to discuss the requirements for a model based framework for the formalisation of risk management and propose a framework which fulfils the requirements.

The chapter begins by defining the requirements for and choice of formalisation framework. It continues by providing reasoning behind the choice through the use of some basic concepts and their relationships to specific applications.

The Unified Modelling Language (UML) is selected and introduced as a high level formalisation tool. To show that the UML can be used as a tool for providing a formalised view of risk management a number of existing applications of the UML are identified and the role of the UML within the application discussed.

The first group of applications is taken from the IT/IS domain, the UML'S domain of origin, showing the breadth of application within its domain. Next, various applications from a group of domains not traditionally associated with the use of the UML are discussed, The aim is to show that the UML not only applies to IT/IS but it has also been applied to the formalisation of other domains.

Each existing application will be related to a number of systems concepts to show the breadth and depth of the use of the UML. 


\subsection{Technique selection}

To provide a consistent understanding of risk management it is desirable to have a common approach to the consideration of all concepts being investigated and defined.

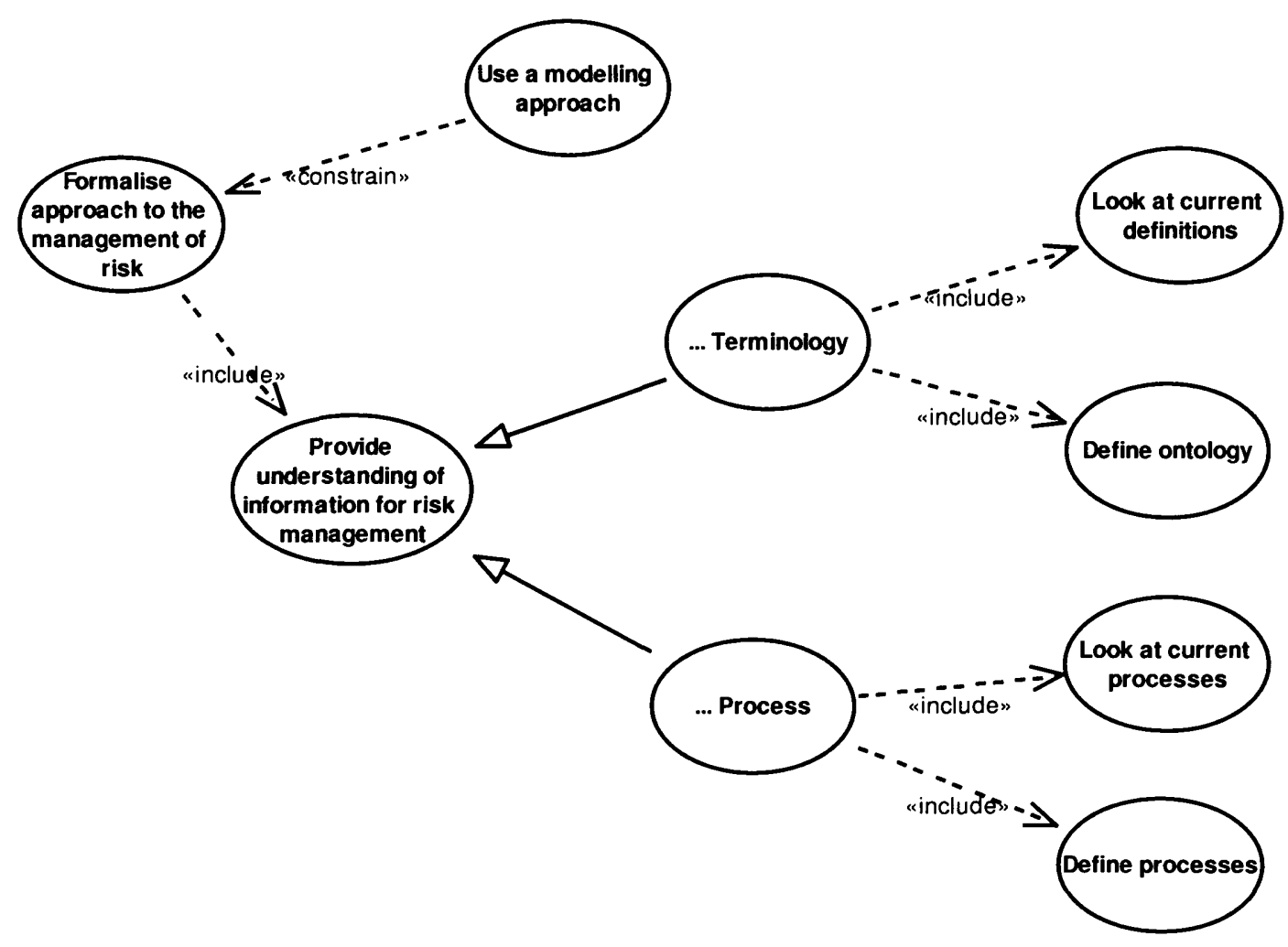

Figure 4-1 - Requirements

The framework selected to carry out the formalisation of risk management must:

- use a modelling approach

- be capable of providing an understanding of the current terminology of risk management

- be capable of providing an understanding of

- be applicable to the definition of ontologies and taxonomies and

- be an accepted approach for the definition of processes. 
These detailed requirements abstracted from the leaf use cases in Figure 4-1 describe the uses of the framework. The framework itself must still fulfil the needs of formalisation including enabling, consistency, repeatability, multiple views and pragmatism.

\subsubsection{Framework Selection}

Options for the provision of the framework required will now be considered. These options focus on modelling languages as languages provide an abstraction from specific applications.

Table 4-1 lists 10 graphical modelling languages showing their ability to model current terminology and processes, define terminology and processes, and provide a formal output. 
Table 4-1 - Framework Options

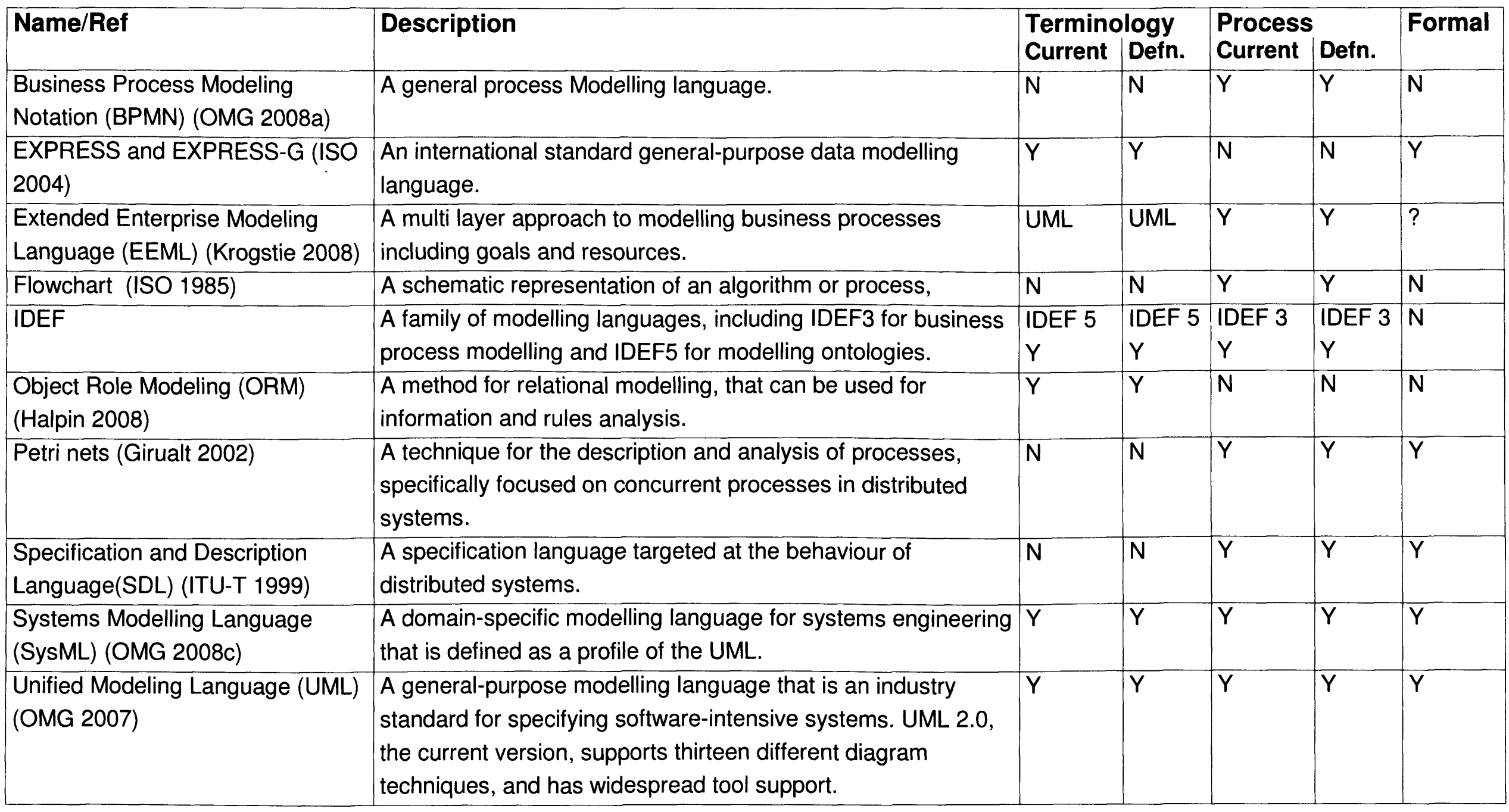


Based on the information shown in Table 4-1 the UML and SysML are the only languages able to fulfil the requirements stated above, specifically the ability to define both process and ontology. As the SysML is a profile of the UML with some specific additions it is possible to select both. The main work of defining ontology and process will be carried out using the UML and use concepts from the SysML where relevant.

To further support the use of the UML a number of example applications have been investigated. These example applications have been categorised by industry and are related to a general set of terms which can be used to describe concepts within a systems understanding.

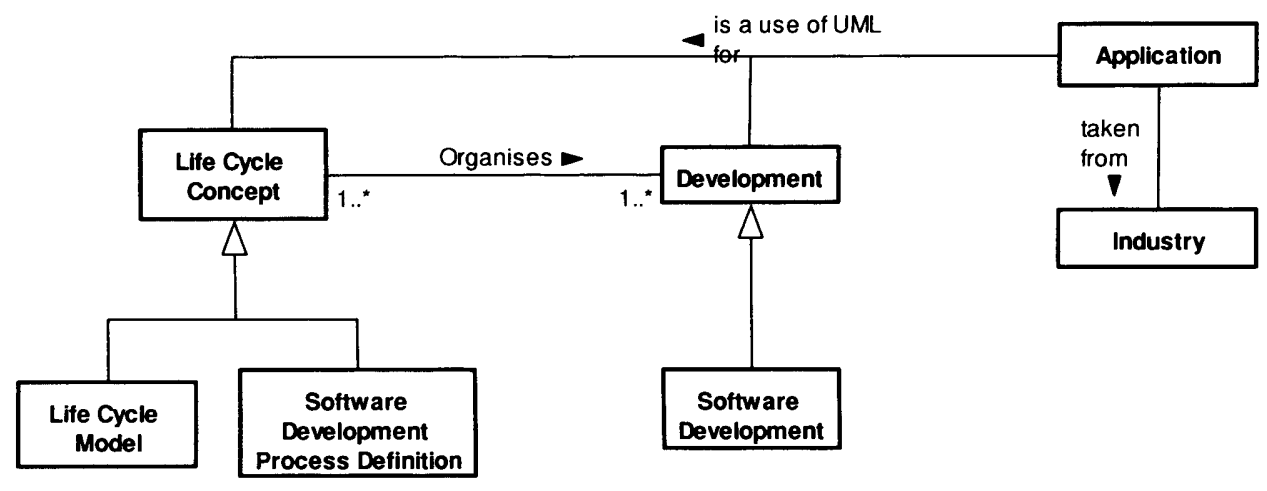

Figure 4-2 - UML Concepts Usage view

The diagram in Figure 4-2 shows the basic concepts to which this chapter relates the applications it describes. The major concepts are Life cycle concept and Development. The Life Cycle Concept provides all of the organisation tools including life cycles, life cycle models and the processes which are executed within them. In this diagram the Software Development Process Definition has been shown as software is the recognised origin of the UML. The need for the Life Cycle Concept lies in the need to organise the work being carried out, this work has been captured with the use of the term Development. Development in this case is the activity of the people carrying out the work whether organised by life cycle and process or not. 


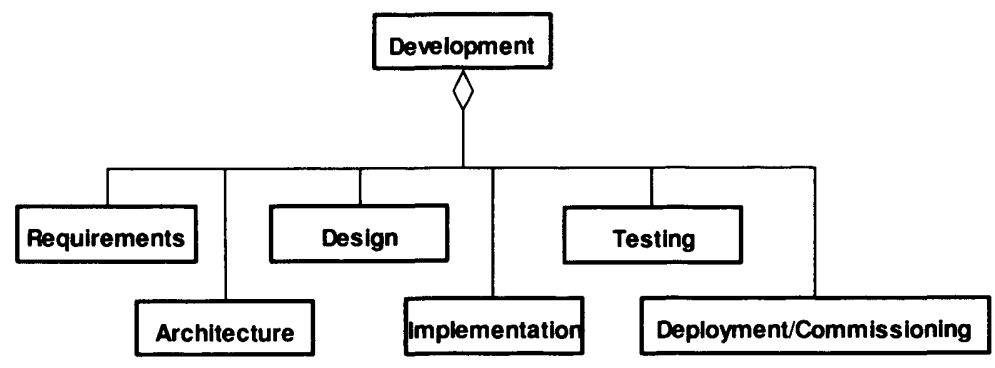

Figure 4-3 - Development

Development can be considered a wide concept, a statement with which NATO agrees based on its definition of the software development process, from their standard AQAP-150 (NATO 1997), the process by which user need/requirements are translated into a software product. Somerville (2007) suggests that software development is where the software is designed and programmed. However he surrounds this definition with the concepts of specification, validation and evolution.

Using these definitions as a basis a set of activities likely to occur within a development has been proposed in Figure 4-3. These abstracted activities are; requirements, architectures, design, implementation, testing and deployment. The activities may sound like processes or life cycle phases: in this case they represent the natural practices which people will carry out even without a process or lifecycle in place.

These two diagrams showing the concepts and the activities within a development can be used together to relate example applications to the life cycle concepts and development. The examples will firstly be taken from the IT/IS domain followed by defence, rail and then science/education each application will have a brief explanation outlining its work and highlighting the areas that were aided through formalisation using the UML.

The first area to be investigated is the IT/IS domain. It has been named IT/IS to ensure that some of the wider issues associated with Information Systems can be captured as well as those associated with the technology itself. 


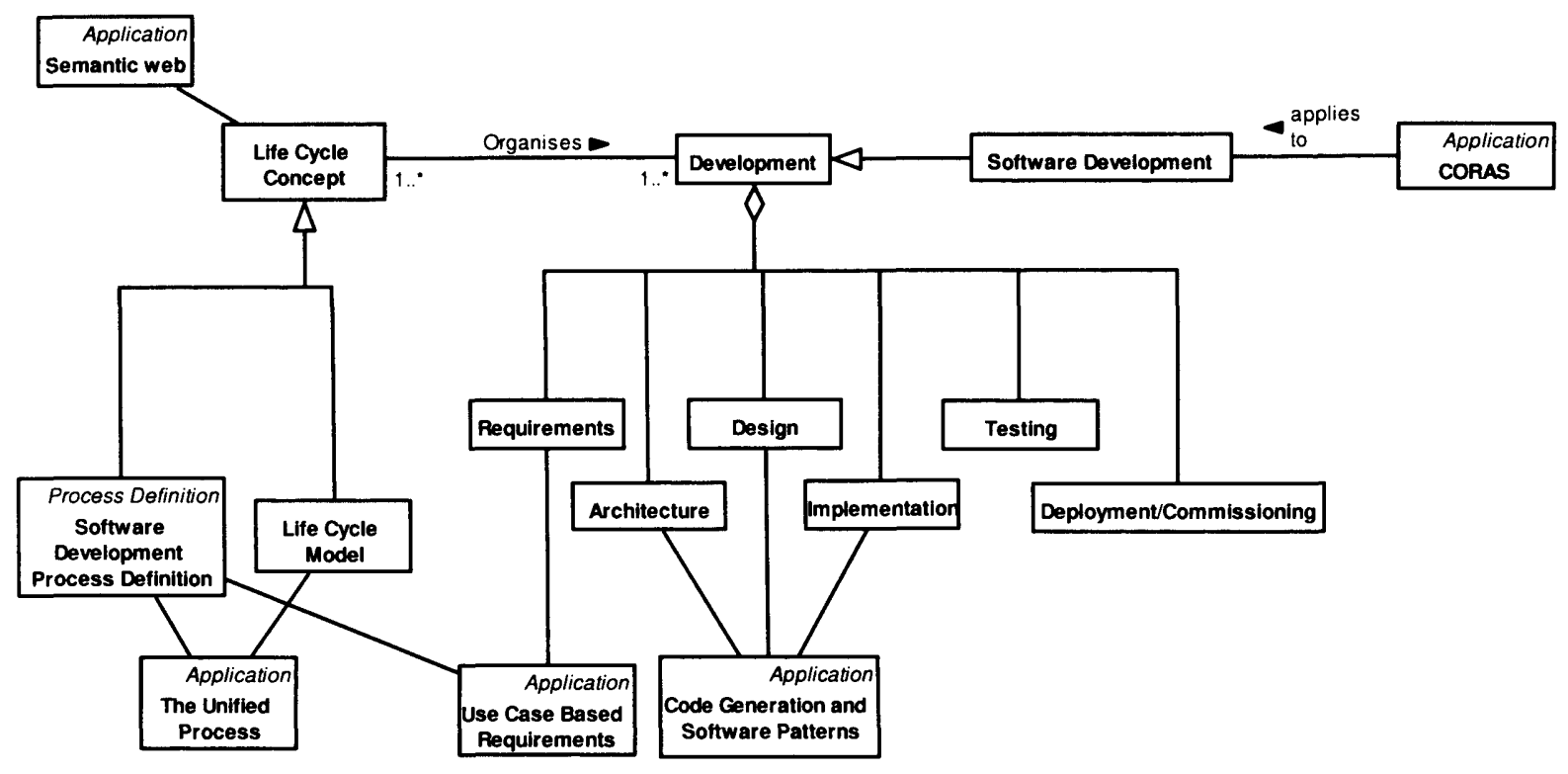

Figure 4-4 - UML IT/IS Usage view

Four IT applications of the UML have been considered including associations with the life cycle concepts and development activities. It can be seen in Figure 4-4 that these four applications apply to different aspects of the development and Life cycle concepts the main points from each application are detailed below.

Code Generation and Software Patterns:-Peckham and MacKellar (2001) used the UML to record design patterns from the database community, once recorded the known good patterns were incorporated into high level conceptual models for new software. The re-use of design patterns enabled speedier design and implementation.

Semantic web:- Baclawski et al (2001) investigates the use of the UML as an ontology development language by comparing it with existing markup languages. He concludes that incompatibility issues can be resolved through the definition of a UML profile.

The Unified Process:-The Unified Process (Jacobson et al 1999) defines a Life Cycle Model as an iterative and incremental model meaning that the stages in the life cycle are carried out once, with the processes being run 
many times within each stage.

Use Case Based Requirements:-Some (2005) defines an approach using Use Cases along side a number of domain models 'Class diagrams' to provide a formalisation of the requirements engineering process. This has been carried out to improve the link between customer need and the system design and implementation.

CORAS:-The CORAS project (Vraalsen et al 2004) applied the UML to risk analysis of security-critical IT systems and provides a tool-supported methodology for model-based analysis. This tool has been designed to apply across all development activities.

From these examples it is reasonable to conclude that the UML is accepted across the IT/IS domain as a tool which provides a level of formalisation and consistency which is not inherent in other system definition tools.

\subsubsection{Application by Industry}

This section provides a number of non-IT/IS applications of the UML. For each example it describes how the UML has been used to aid in formalisation, consistency and communication.

The first of these areas is defence where, with so many organisations contracting for and supplying equipment, a clear consistent approach to communication and system definition is imperative. 


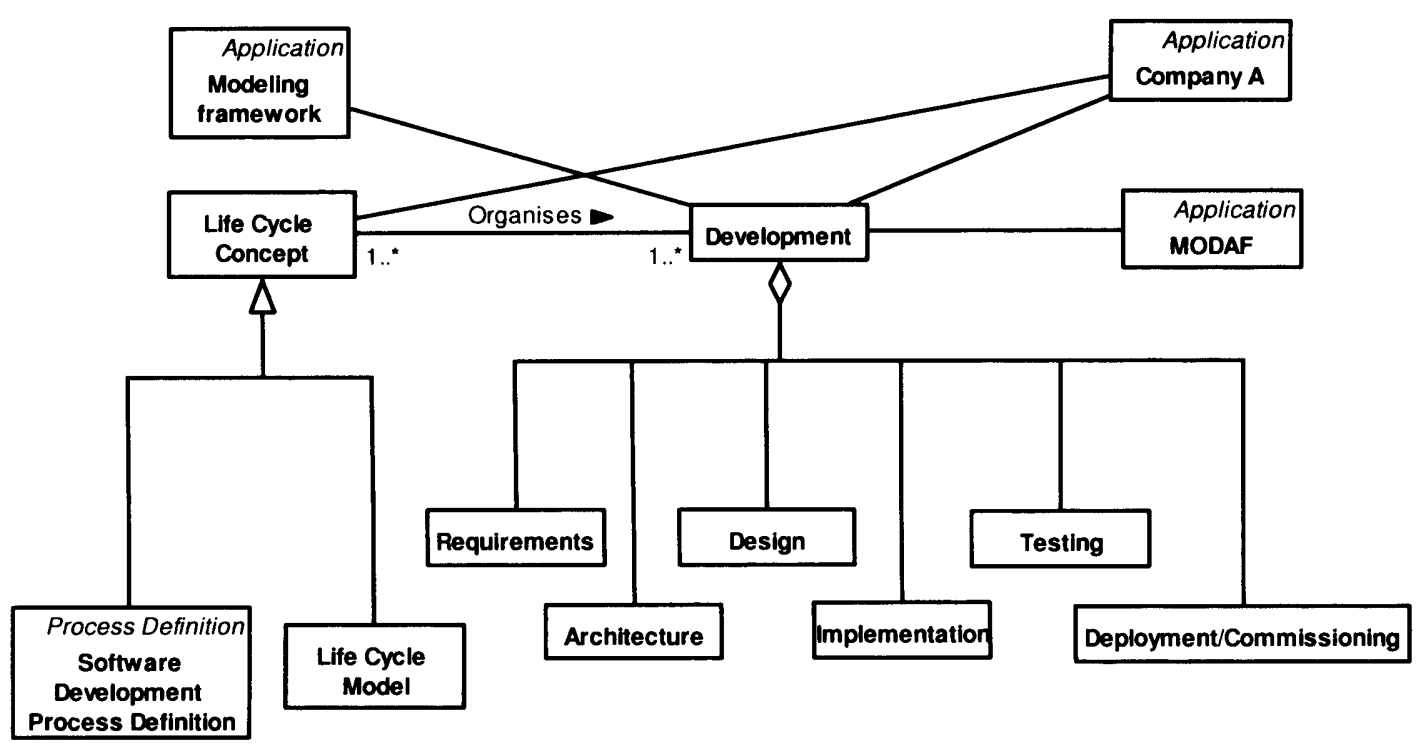

Figure 4-5 - UML Defence Usage view

It can be seen from Figure 4-5 that three defence applications have been considered. These applications apply to different aspects of the development and Life cycle concepts the main points from each are detailed below.

Company A:-Company A uses the UML to define its life cycles and processes. When following the processes for system development all relevant information and artefacts are also developed and delivered through the medium of UML. This company is doing this to improve its Systems Engineering capability. It sees the use of the UML as providing consistency and formalisation to the work they are carrying out.

MODAF:-MODAF (2007) provides an Architectural framework for the UK Ministry of Defence (MOD). This framework is used to format information which in turn supports communication between the MOD and its suppliers. The MOD have suggested that the UML and SysML can be used to deliver a number of the views within the framework due to the level of formalisation offered by the UML.

Modeling framework:-Nicola et al (2007) discusses the Conceptual Modeling Framework-Ontology, this discussion is included as a chapter in the book Enterprise Engineering 
The use of the UML in military acquisition shows that there is an appreciation of the breadth of application which the UML can have. It is not only being applied to IS/IT projects but to any system delivery project within the MOD.

The rail industry has been an established industry for over 200 years. The safety culture that comes with this industry was not far behind. The reasons for this culture as discussed by Faith (2000) include a long history of rail accidents. As the world moves forward with both technology and expectation the rail industry must also improve.

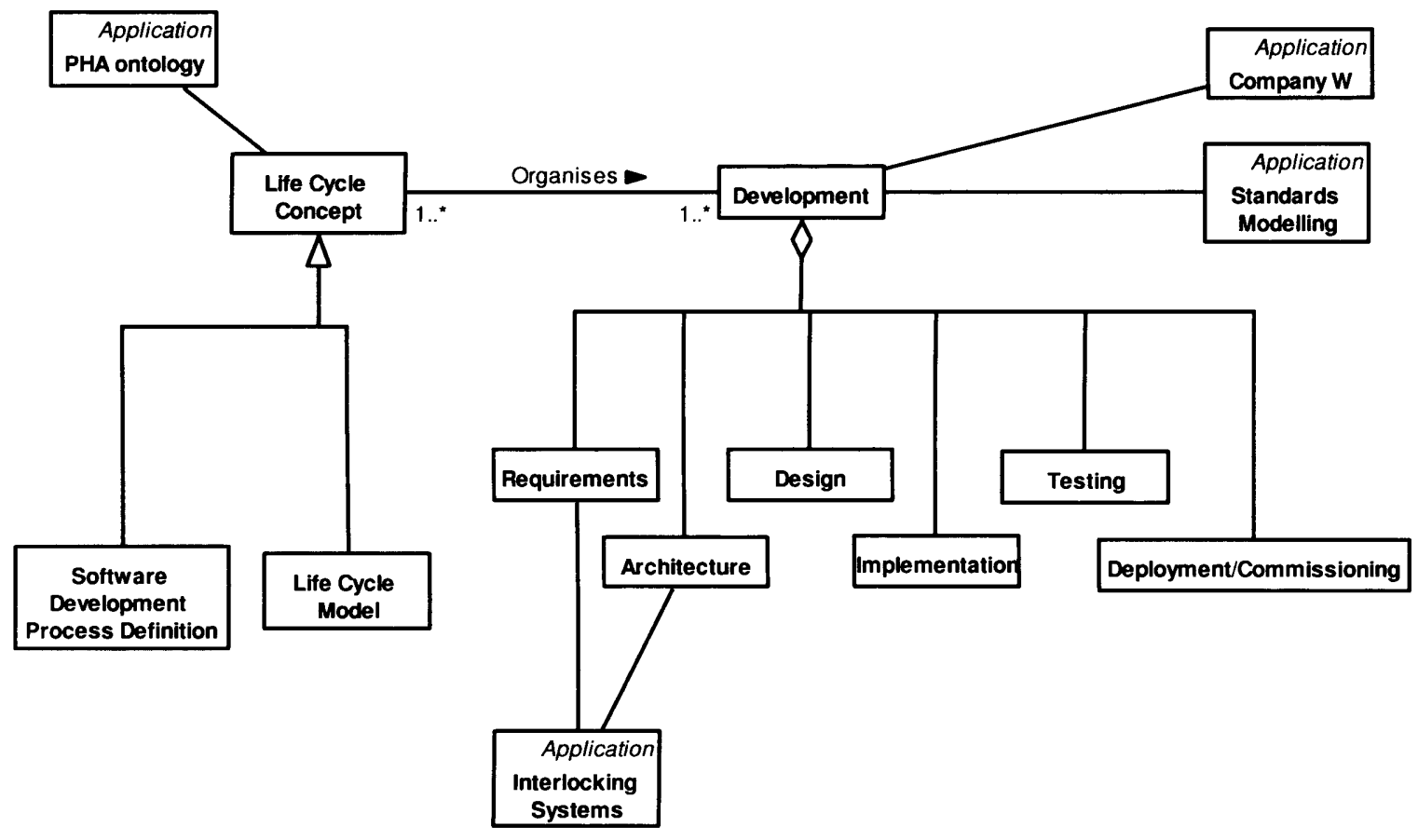

Figure 4-6 - UML Rail Usage view

This work has considered three example applications from the rail industry. It can be seen from Figure 4-6 that these applications apply to different aspects of the development and Life cycle concepts.

Company W:-Company $\mathrm{W}$ use the $\mathrm{UML}$ for their system development this enables them to produce the minimum number of external artefacts by holding all of the system information in one central project repository. 
Interlocking Systems:-Modelling Interlocking System Requirements in UML (Bayley 2004). Interlockings are the safety system behind railway signalling. Their purpose is to inhibit a situation where two trains could be in the same place at the same time. Bayley discusses an abstract model of the European Rail Traffic Management System (ERTMS) which can be simulated at the requirements level. This work enabled communication through the formalisation of the understanding of the ERTMS

PHA ontology:-Mazouni and Aubry (2007) uses the UML as a tool to define an ontology for Preliminary Hazard Analysis (PHA). This provides terminology which may be used to describe accident scenarios, risk calculation, severity calculation and risk reduction.

Standards Modelling:-Barrow (2005) applies UML to the modelling of standards to show the benefits that can be gained through a more formal structure and common communications medium. In this example standards modelling was applied to train activated warning systems and ERTMS.

The UML has been used in the rail industry to improve clarity, abstract multiple views and improve communications with suppliers effectively shortening supply time.

Science and education are aimed at formalisation, understanding and teaching. Bloom (1956) made a large contribution to this when they developed their taxonomy of education which sets out a number of levels of learning along with learning domains. 


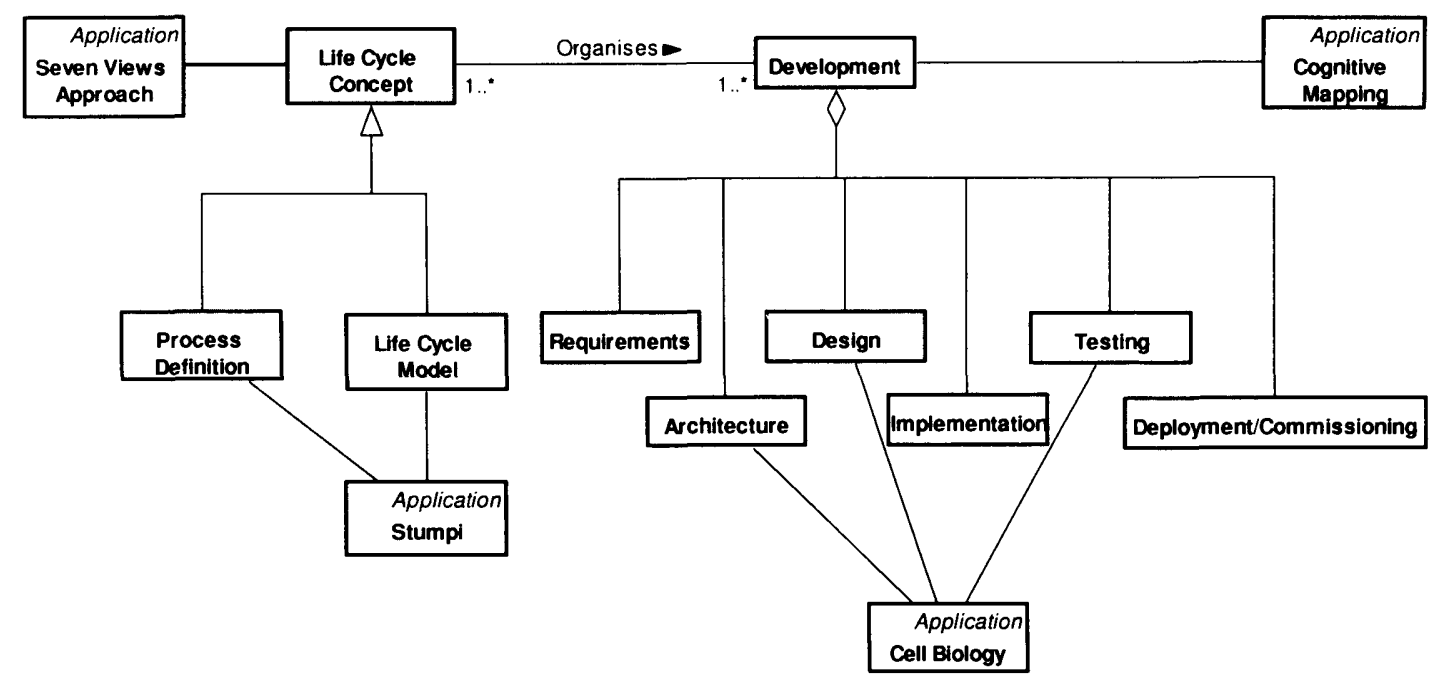

Figure 4-7 - UML Science/Education Usage view

Four Science and Education applications of the UML and their associations with the life cycle concepts and development activities can be seen in Figure 4-7. These applications apply to different aspects of the development and Life cycle concepts the main points from each are detailed below.

Cell Biology:-Webb and White (2005) used the UML to develop models and improve understanding of Cell biology

Cognitive Mapping:-McNellis (2005) used the UML to represent cognitive mapping methods improving the consistency within the maps.

Seven Views Approach:-The seven views approach to process modelling defined by Holt (2005) is adopted by the BSI as the best practice approach for modelling processes. This approach, defined using the UML, provides a formalisation and completeness to process modelling.

Stumpi:- Holt (2004) uses the UML to provide a tailored life cycle complete with life cycle processes. This approach enables university students to understand the importance of Life cycles and processes before carrying out their degree projects following a defined life cycle model. 


\subsubsection{Application Overview}

When considering all the applications discussed in one view the breadth of application, based on basic concepts, can be appreciated.

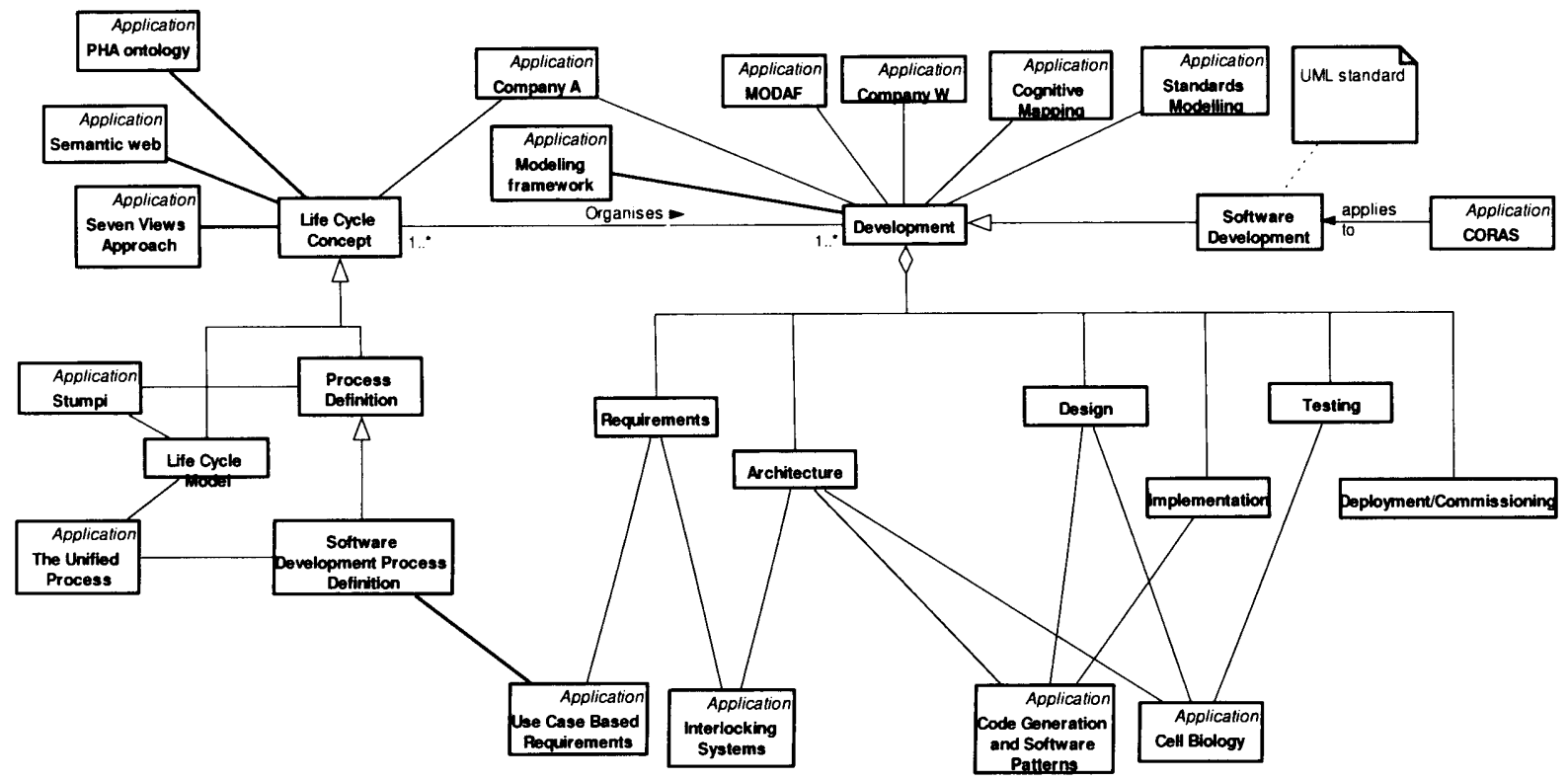

Figure 4-8 - UML Developed Usage view

Figure 4-8 is based on only the applications from the four domains discussed, however it provides an overview of the breadth of application of the UML in its use as a formalisation framework. It has shown that the UML can be used to define both terminology and approach.

The UML provides a multi view language based on the use of up to 13 different types of diagram. The diagrams are inter-related, the relationships provide the ability to carry out consistency checks between diagrams enabling confidence in the concepts defined to grow. Both views and consistency are routed in an object oriented approach. Object orientation itself is not a concept which has been defined to aid in software development. It can be traced to Descartes thinking on human perception, well before software was considered. The use of object orientation providing multiple views, consistency and repeatability enables the UML to be used as a framework for formalisation. The following sections describe the way in which the UML will be applied to the visualisation and formalisation of ontology and process. 


\subsection{Ontology Modelling}

Examples of domain specific uses of the UML for defining ontologies have already been shown in the applications above. What is required in this case is an approach which in not related to domain.

IDEF 5 (1994) provides a generic approach to the definition of ontology using its own schematic and elaboration languages. The schematic language within IDEF 5 enables the definition of initial visual versions of ontologies however, it provide a large number of detailed constructs which could be considered too complicated for any initial version. The IDEF approach provides three schematic views Classification, Object state and Composition along with symbols which can be deployed onto the schematics. The main symbols are Kind, Individual an instance of a kind, process and relationship it goes on to define many types of relationship covering state relationships to physical parts.

Others including Cranefield and Purvis (1999) have been investigating the use of the UML as an ontology modelling language. Cranefields approach is to use the UML to describe an ontology and compare this with the advantages and dis-advantages of existing ontology representation languages used for knowledge based reasoning. Cranefield uses UML class and object diagrams to obtain what he describes as "both a highly structured model that could support automated reasoning and an expressive language that it would not be practical to attempt general-purpose reasoning wtih."

The approaches taken by Cranefield, use of class diagrams and IDEF5, definition of kinds and relationships, can be abstracted to fulfil the generic needs of defining an ontology, which is not focused on the use of automated reasoning, using the UML. 


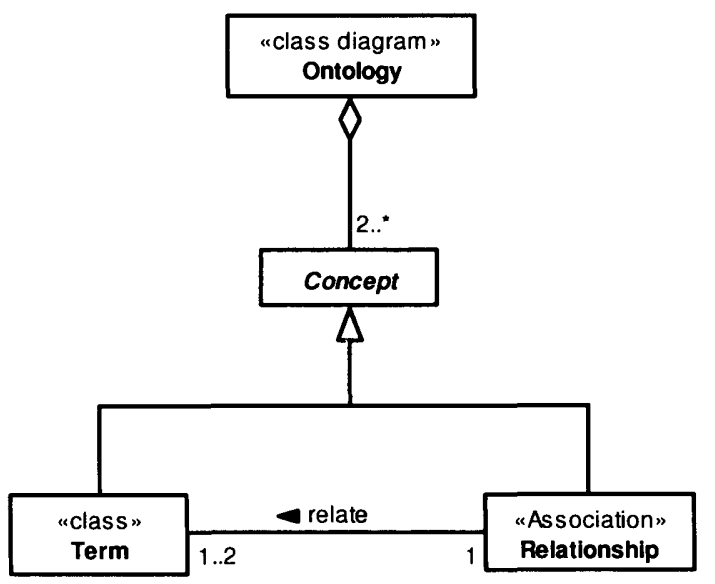

Figure 4-9 - Ontology concepts \& realisation

A generic definition of an ontology, as presented in Figure 4-9, shows Concepts which may be Terms or Relationships: the relationships relate terms to each other. The stereotypes, shown within the chevrons, define the UML elements which will be used to represent these Concepts on an Ontology.

\subsection{Process Modelling}

The approach to defining an ontology discussed above will provide an understanding of the concepts of risk and its management although alone they will not be enough. An Ontology will not explain how to carry out risk management as it only defines the terminology to be used in it as suggested by Spies (2006).

To provide an approach to the management risk we must define the behaviours and artefacts. The best way to achieve this is through the application of a process modelling technique. A number of techniques exist including BPMN (OMG 2008) and IDEF3 (1995) however, neither of these use the UML and therefore would reduce the ability to ensure consistency within the formalised approach.

Holt (2005) has provided an approach to using the UML as a process modelling approach known as the Seven Views Approach. Perry (2006) has conducted a comparison between the Seven Views Approach and the BPMN 
showing that the approach covers all of the views from the BPMN and provides extras to ensure that a consistent model can be created.

This section will provide an overview and introduction to the Seven Views Approach by presenting the concepts behind the approach and the way in which it is realised in the UML.

\subsubsection{Process Concepts}

There are three main concepts involved. The first is the Source, namely where the process knowledge is held. Knowledge may be tacit or previously recorded. The second Presentation; is the way the process is delivered to the end user. The presentation may have to vary for users who have different reasons for looking at the process. The third and possibly most important is Understanding this is where the process knowledge is captured and interpreted to develop a consistent and complete model of the process.

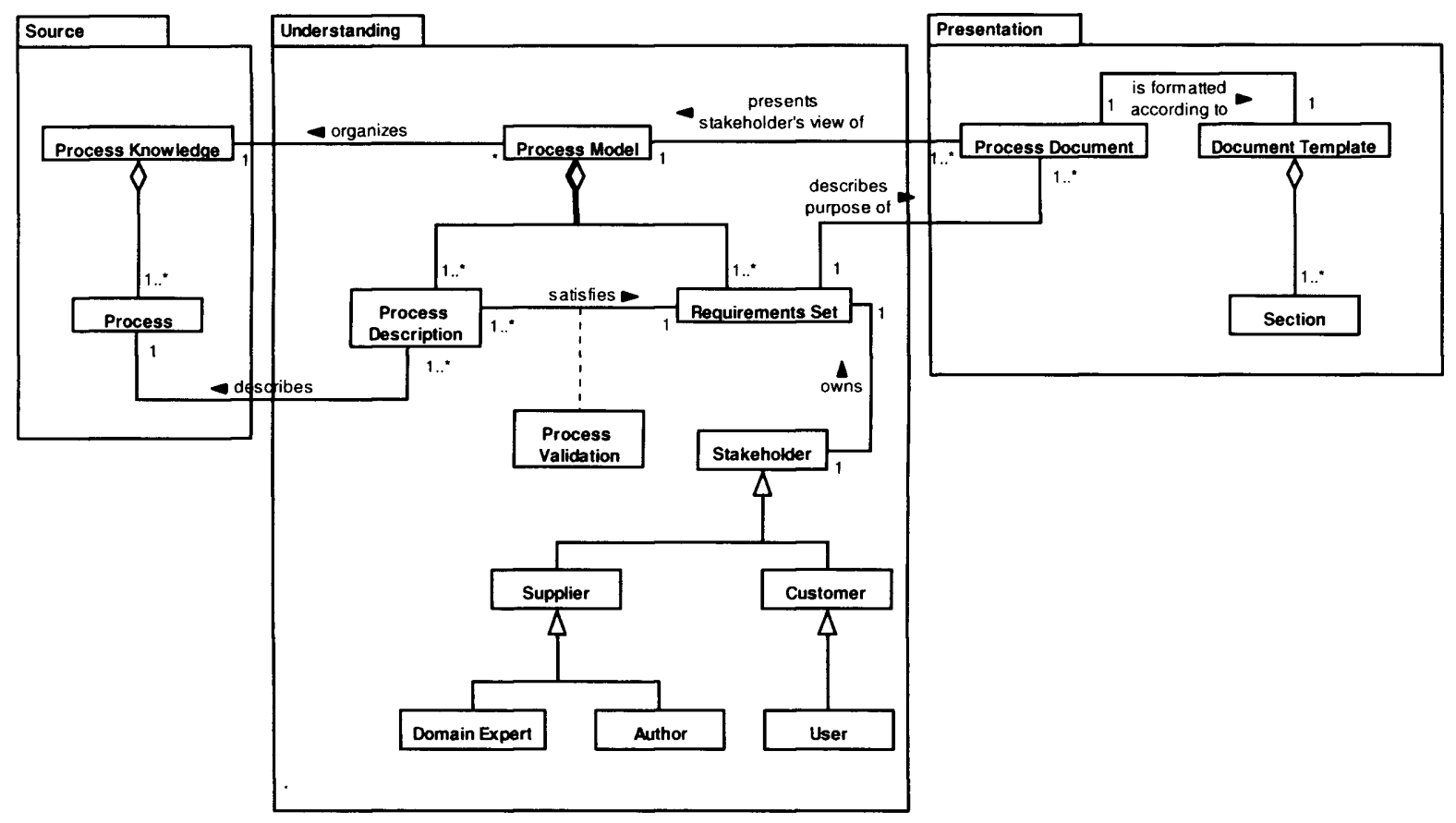

Figure 4-10 - Process Concepts View (Holt 2005)

The problem that occurs is the way that the Source and Presentation are related. In most cases, the person who has the tacit knowledge of the process records what they know and this record becomes the presentation. This is where the Understanding becomes important, to fully understand the process 
knowledge one needs a model to organise the thoughts, this model must explain why the process is needed and provide a clear and consistent description of the process.

The realisation view further expands the description of the process; it also relates the process modelling terminology to the concepts from the UML that will be used to realise them. The main advantage to this approach is that it is not relevant where one starts collating information. The fact that the relationships have been navigated is the most important point about this model as navigating the relationships ensures a complete and consistent model for the process.

The realisation view provides the overview of the seven views along with a number of the relationships which provide consistency when building up the detail within the views.

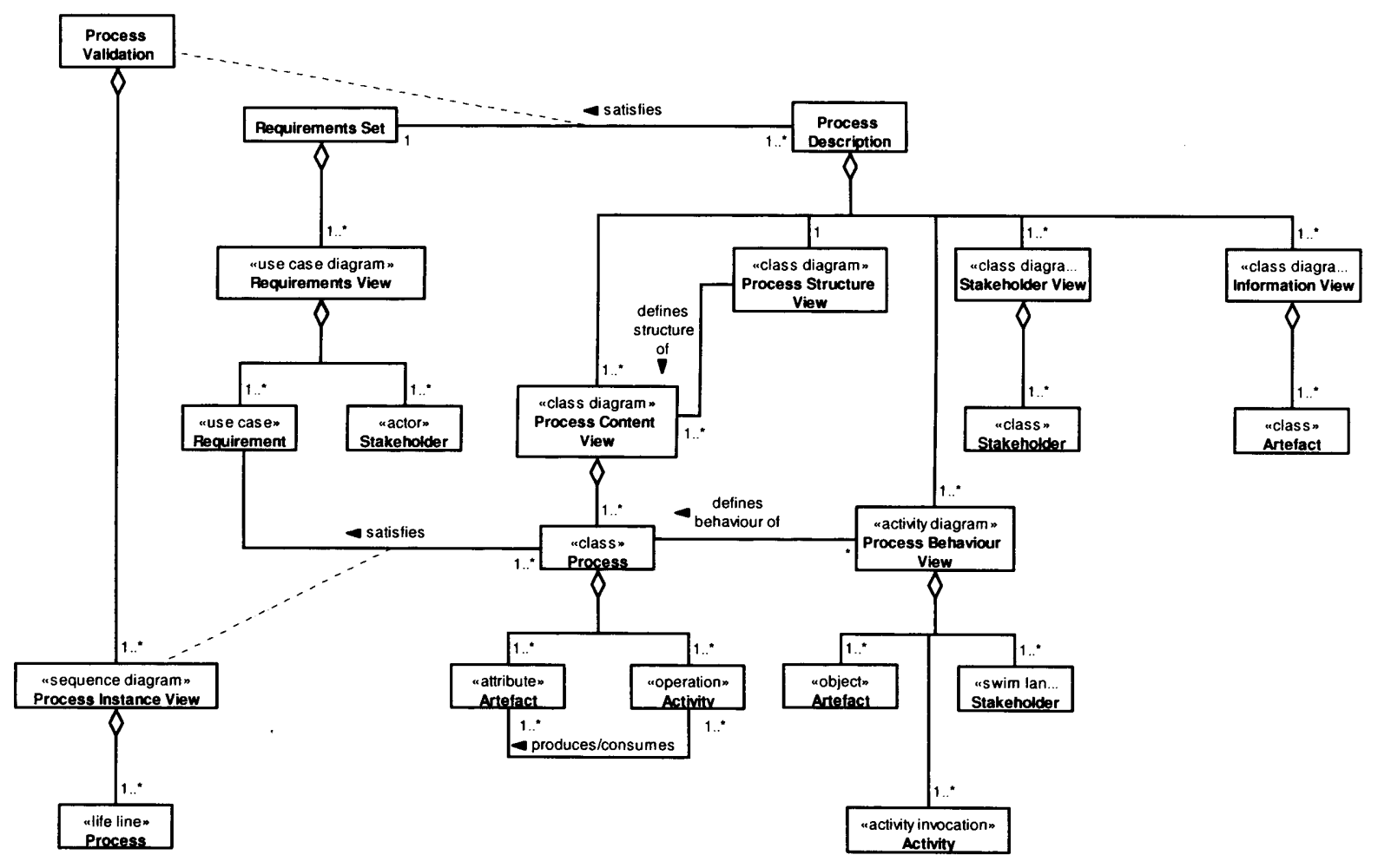

Figure 4-11 - Process Realization View (Holt 2005)

A full picture of the process can be developed by producing these seven views (Figure 4-11): 
The Requirements View presents the need for the process: this can be very useful for checking whether a process is still relevant or requires updating as the needs may change over time.

The Process Content View provides a static view of the process, it shows the Process along with the Artefacts which are used and produced by the Process and the Activities which use and create the Artefacts. This view can be very useful in compiling a library of processes.

The Information View shows the relationships between the Artefacts within a Process: it can also be used to show the relationships between Artefacts in different Processes. This is very useful for understanding the documentation required from the process and provides a powerful method of reviewing the documentation to eliminate replication of documents, remove unused documents and show documentation updates over time.

The Process Behaviour View is the view of the process which everyone expects to see first and is the main view presented by BPMN (OMG 2008). It shows the order in which the Activities are carried out, any decision points within the process and where the Artefacts flow. It can also be used to show the Stakeholder responsible for ensuring that the Activities have been carried out.

The Stakeholder View presents the roles to be fulfilled in relation to the process, these will be consistent with the Stakeholders on the Process Behaviour View and Requirements View.

The Process Instance View is used to show the order in which the processes can be run, from a theoretical point of view, or have been run, as a way to record their execution on a project. Process Instance Views are also used in the development of project plans ensuring that the project relates to the processes which exist within an organisation. 
The Process Structure View shows the structure and terminology to be used within a process for an organisation. It can also be used to show the relationship between processes and life cycles.

In this work the basic structure shown in the diagram above will be used as the Process Structure View meaning that a Process will always be made up of Artefacts and Activities.

\subsection{Conclusions}

This chapter has discussed the breadth of application of the UML as a formalisation tool, presented an ontology for risk, a set of definition mappings to show the relationships between the ontology and existing standards and shown an approach to process modelling.

The applications of the UML have shown both breadth of usage across the IT/IS domain as well as three other industries, education, rail and defence. It has also shown a depth of usage with some applications applying to the whole development and others to specific aspects within. On the whole this chapter has shown that the UML can be used as a formalisation tool in many domains and as it provides a consistency within the language, improved communication and reduction in complexity.

The objectives of this chapter were to discuss the requirements for a model based framework for the formalisation of risk management and propose a framework which fulfils the requirements.

This chapter has achieved these objectives by defining the needs of a formalisation approach, selecting the UML as the formalisation framework, showing that the UML can be used in many and varying applications and as such is relevant for both ontology and process modelling, it has also described the way in which the UML will be used to model both ontological elements and processes. This work will continue by defining an ontology for risk management using the UML as a definition medium. 


\section{Ontology}

\subsection{Introduction}

The previous chapter selected the UML as an appropriate tool for the defining ontology, taxonomy and processes. The literature review, Chapter 2, showed the lack of consistency in terminology from national and international standards.

The objective of this chapter is to use the UML to define risk and present an ontology for risk management. This means defining the terms which can be used in the context of their relationships to other terminology. This will be achieved by firstly defining a risk and then widening the scope and incorporating some of the wider terminology which is associated with a risk.

\subsection{Risk Definition}

The following section presents a formalisation of the terminology and concepts of a risk, including a definition of a risk. Once defined this definition of a risk will be compared with a number of definitions discussed in chapter 2 before presenting some advantages of this definition.

The section will be concluded with a description of an ontology for risk showing the relationships between the terminology discussed.

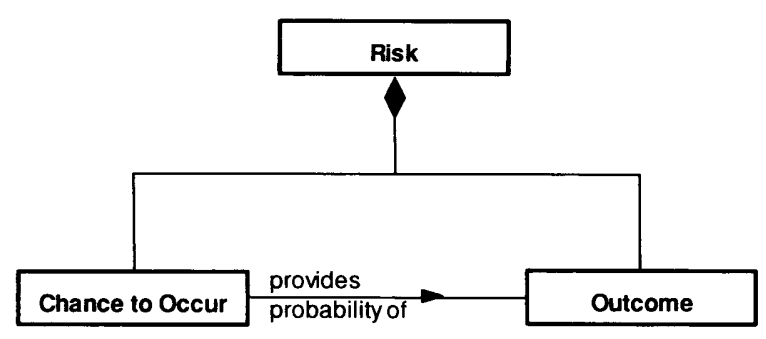

Figure 5-1 - Generic risk

This abstracted definition (see Figure 5-1) has been written to apply to any situation or industry. In contrast most of those discussed in chapter 2 are industry specific. 
The definition shows that for a risk to exist there must be a Chance to occur and an Outcome. The Chance to occur provides the probability of the Outcome occurring. The Outcome described refers to the unwanted event. Outcome in many cases including Hollnagel (2008) and Aven and Kristensen (2005) is used interchangeably with consequence.

This definition has been purposely kept simple and, more importantly, singular. The singularity is to provide more clarity and consistency, if discussing a risk then it must be one risk. It would be counter intuitive to then refer to multiple outcomes in a risk. The Chance to Occur is also singular as it is logical that there can only be one Chance to Occur for any one Outcome. This singularity adds an orthogonal view when asking, have all outcomes been considered. For all outcomes to have been considered the sum of all the associated Chance to Occur must equal 1, assuming that Chance to Occur is presented as a probability. This suggests that a set of risks will be collated creating a 'Risk Set' which itself would need to be verified. A full investigation into the verification of completeness of a 'Risk Set' is beyond the scope of this work.

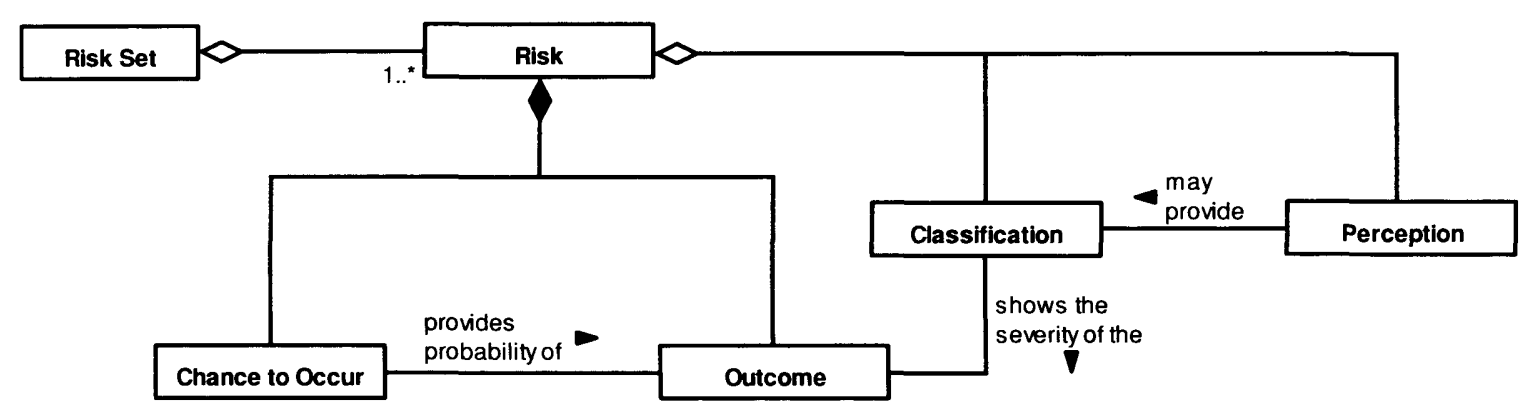

Figure 5-2 - Risk Composition

Once a risk has been defined it is possible to apply a Classification to the Outcome. Classification in most cases is related to the idea of the severity of the Outcome when considering people and injuries, classifications are likely to include Insignificant, Marginal, Critical and Catastrophic. Classifications such as these are often related to the effect on people or a system and tend to focus on industry specific issues Kristensen et al (2006) discusses a number 
of classification schemes. Where people are concerned classification is rarely the only consideration it may also be necessary to understand stakeholder feelings towards the Outcome and Classification these feelings are known as the Perceptions.

Perceptions may help to define classifications but are one of the most dangerous aspects to understanding and treating risk; a perception is a view of the severity of the outcome from a specific stakeholder's understanding, this is discussed in depth by Belzer (2001) in his paper on grin and bear it practices in risk management. Perception is the focus of Pezzullo and De Filippo (2009) paper on emergency management in Hazmat logistics and is alluded to by Clarke (2007) when discussing probabilistic and possibilistic risk.

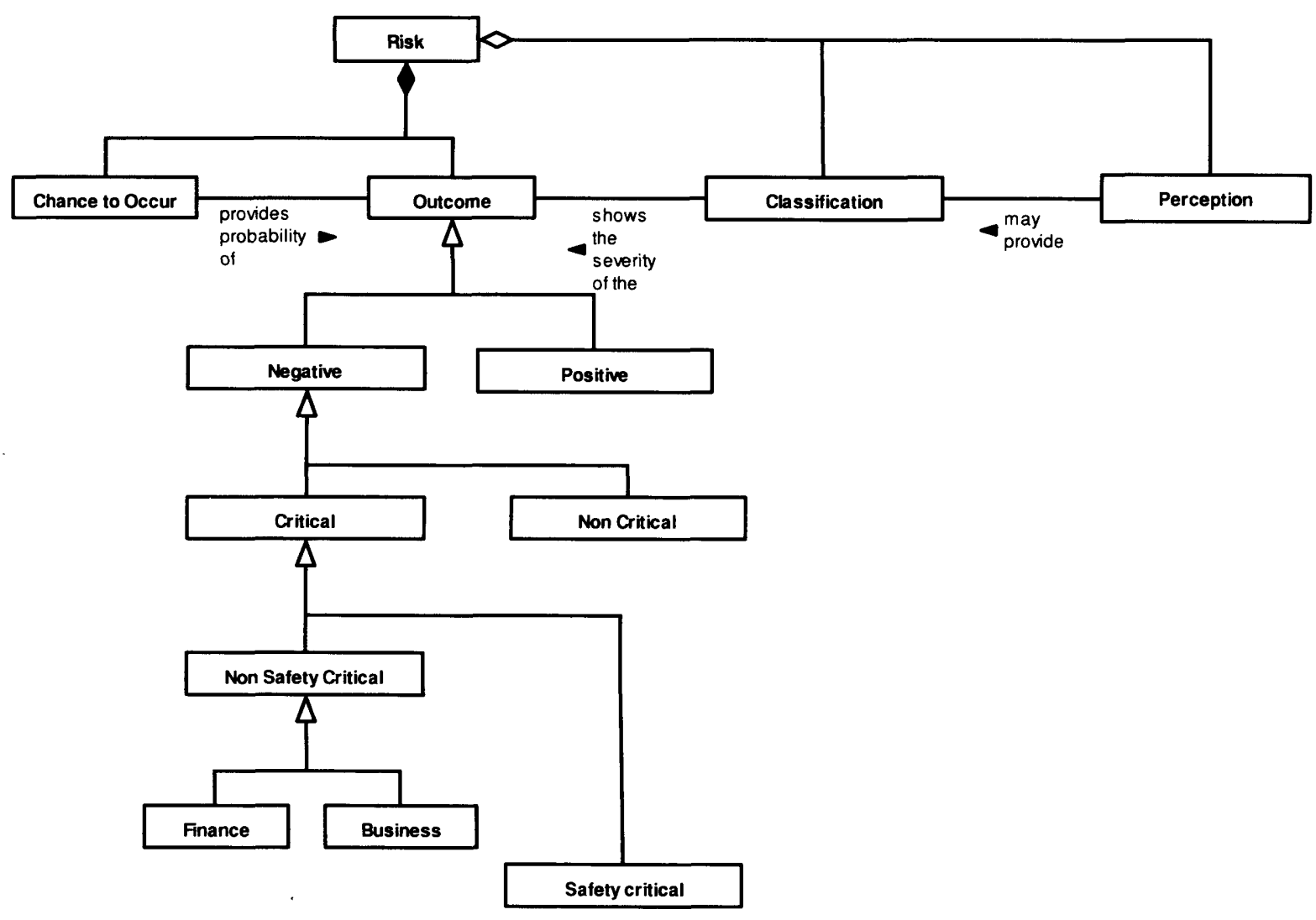

Figure 5-3 - Taxonomy of Outcomes

One approach to categorising systems and the types of risk that relate to them is to categorise the outcome (Figure 5-3) 
- Firstly is the outcome positive or negative? Most will no longer consider an outcome to be part of a risk once they have established that this outcome will benefit them. Hessami (1999) considered this in his paper on Risk a missed opportunity? Also Flage and Aven (2009) discuss the need to balance positive and negative outcomes through the use of portfolios.

- Once it has been defined as negative - is it critical or non-critical? Again non-critical outcomes tend to be forgotten.

- For critical outcomes is there a safety implication? This is where most start to consider outcomes as risks it is also the point at which most standards begin.

In many areas critical systems or safety critical systems are discussed. In these cases it is the Classification of the Outcome which is being used to select the category of risk. In many cases the category will then be transposed onto the name of the system to highlight possible outcomes and therefore the need for a more rigorous approach to the system definition and development.

One problem here is that in many instances there is no differentiation between the categorisation of a system and the classification of an outcome. Classifications may be assumed due to the categorisation of the system.

There is a need to be consistent about classification and categorisation of risk.

- Classification - relates to severity of outcome

- Categorisation - relates to the separating out types of outcome i.e. financial, marketing etc. 


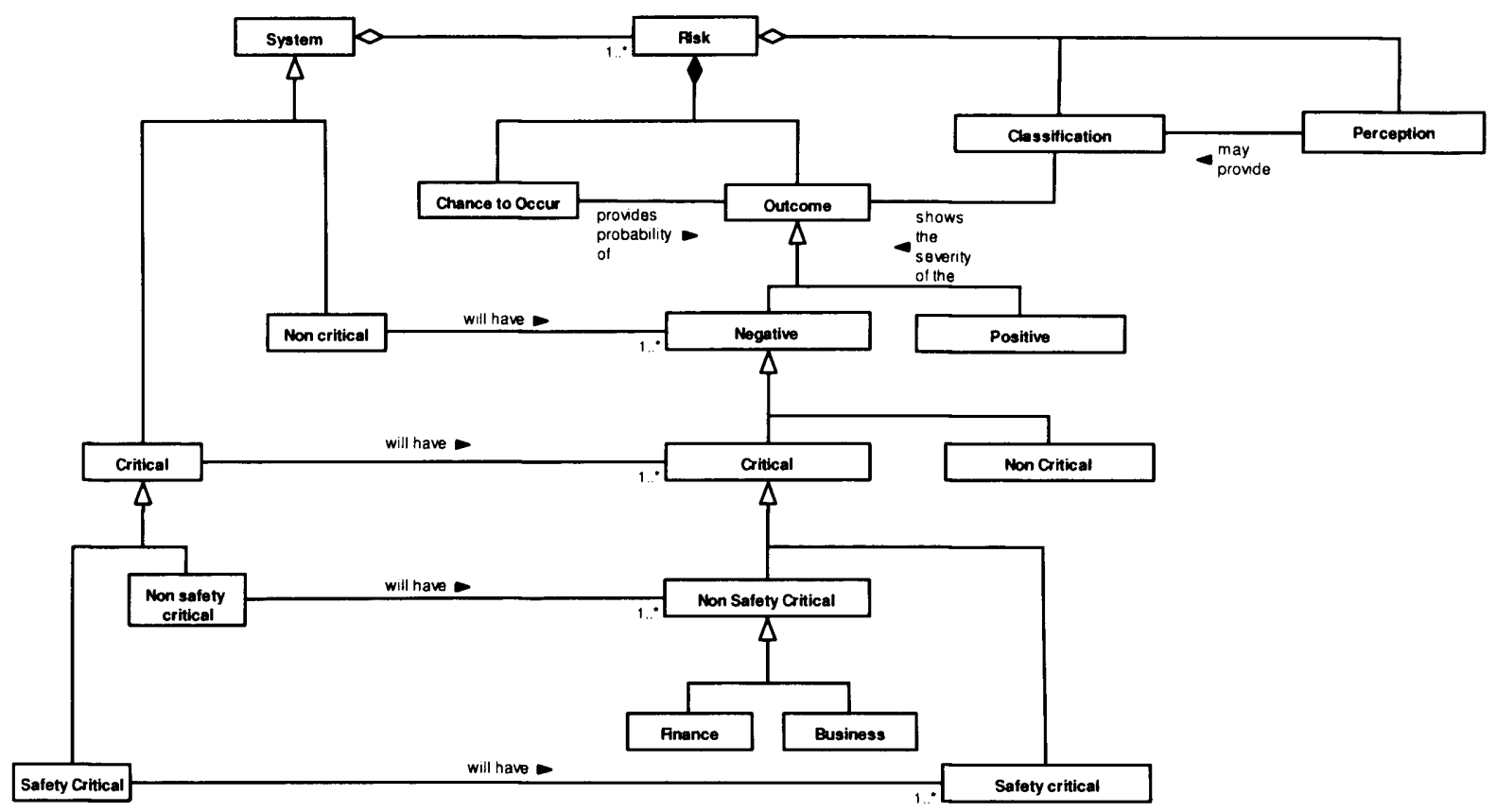

Figure 5-4 - System vs Outcome

This diagram (Figure 5-4) has also linked these categorisations of the outcome to terminology which is used to describe systems - it must be remembered that there is no hard and fast rule as to how a system and an outcome are related, the words are generally used interchangeably and hence, one should be extremely careful with their use.

\subsection{Associated Terminology}

The following defines relationships between risk and some of the broader terms which are often associated and sometimes confused with risk.

\subsubsection{Causal terminology}

This work has not set out to define all of the terminology associated with risk. However, there are concepts which need to be considered to ensure the scope of risk management can be understood, hazard is one of those terms, it has been used here to group the terms from chapter 2 which cover the idea of events leading up to the occurrence of an Outcome. 


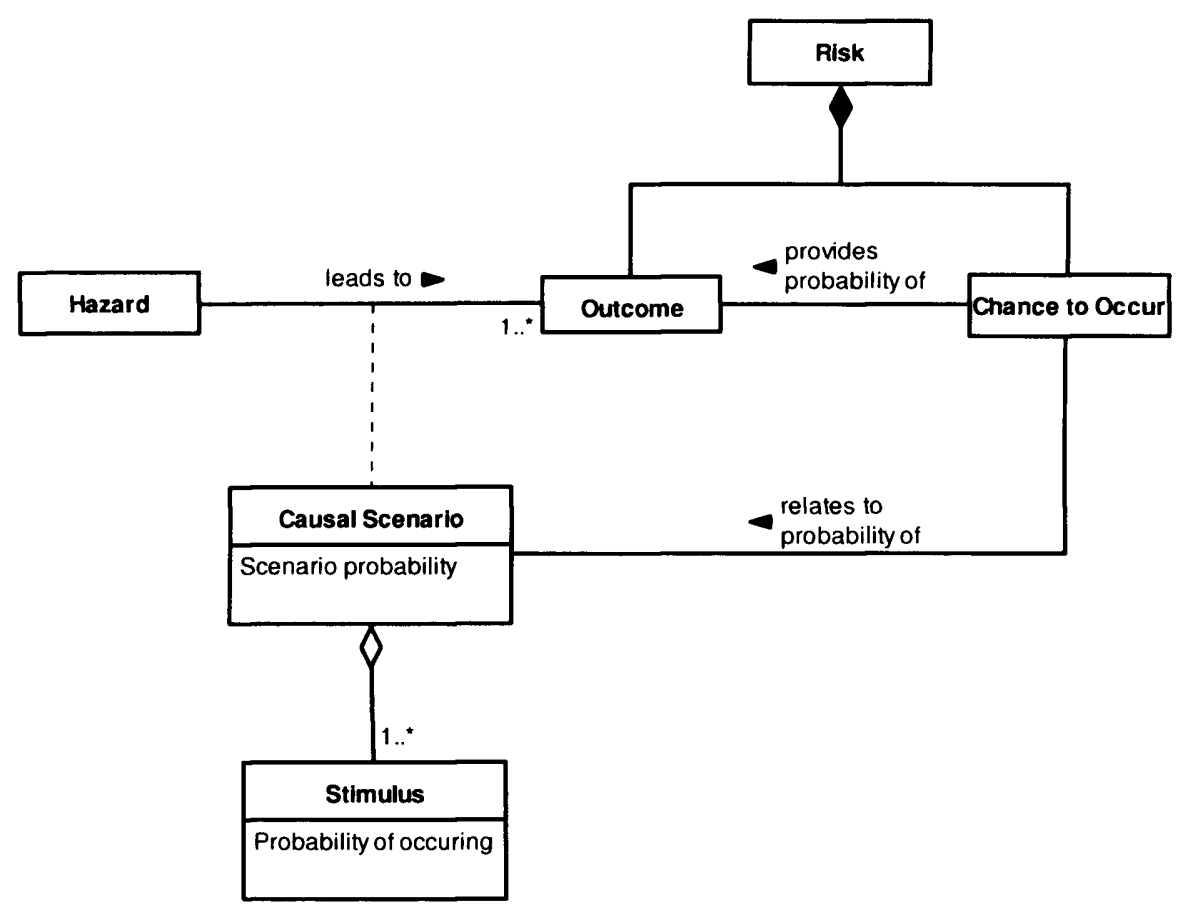

Figure 5-5 - Causal analysis

There are many ways to consider stimuli which precede the outcome defined in this work these will be called Hazards, Figure 5-5. The diagram also shows that the relationship between the Hazard and the Outcome is via Causal Scenarios, the fact that this is a scenario means that there could be many hazards leading up to the Outcome and it is important that these are recorded as they will be required for both causal analysis and definition of mitigations.

The two salient points to be remembered about hazards are that they must be recorded and they must happen before the outcome, the Effect will happen afterwards, this is characterised by the bow tie model (Delvosalle et al. 2005). 


\subsubsection{Consequence Terminology}

Consequences or effects are all of the things that happen or need to happen after an Outcome has occurred.

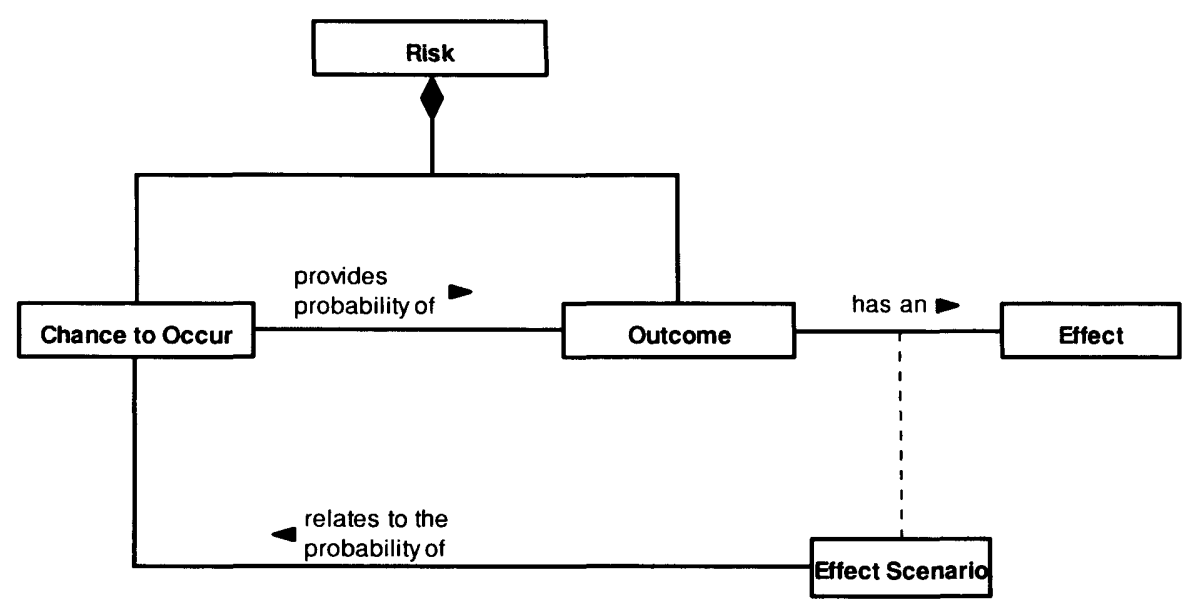

Figure 5-6 - Effect Analysis

Effect analysis is in essence opposite to the Hazard or causal analysis. It provides an understanding of what happens after an outcome has occurred Figure 5-6. Again it is important to record effects as they will, for those Outcomes which can't be removed, become the basis for the policies and procedures acting as mitigation.

\subsection{Mappings}

As discussed in Chapter 2 there are already many definitions of risk; any new definition will need to have a justifiable difference. The difference in this case is that using the UML as a common language highlights similarities and contrast across existing standards and approaches enabling a dialogue to be held between risk experts in different industries. It may also provide a common base knowledge of risk before specialising in one area. This section presents four mappings between the definitions presented in this chapter and those discussed in chapter 2.

Mappings will be separated in to those which map directly to the definition of risk and wider mappings to the associated terminology. The mappings will highlight the differences between the terminology within the definitions which 
had previously been considered to be commensurate.

\subsubsection{Definition mappings}

The definitions which have been mapped include financial, project and technical. The technical definitions cover both safety and non-safety categorisations.

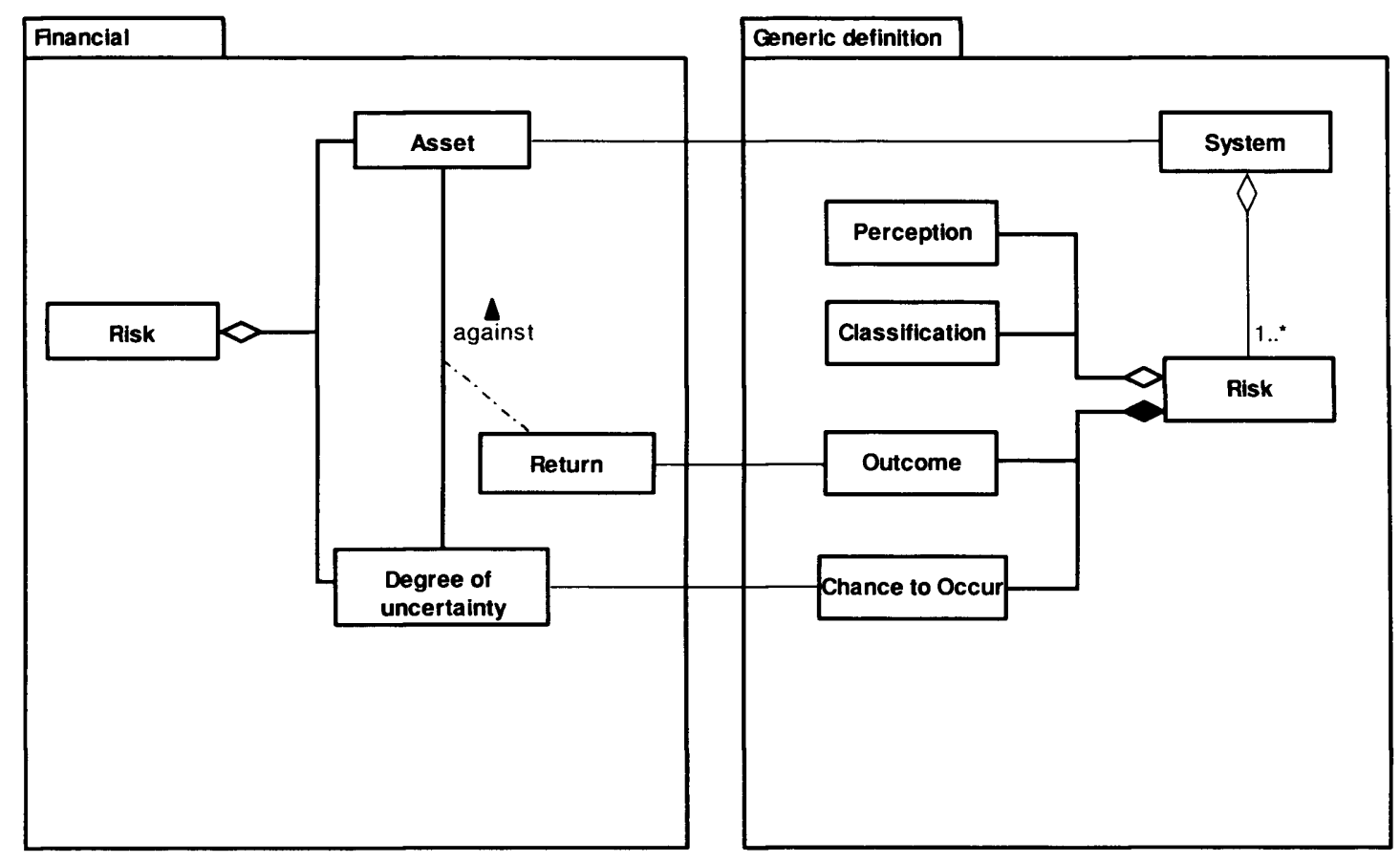

Figure 5-7 - Financial

Two direct mappings can be made from the financial definition, taken from Harvey's financial glossary (Harvey 2002), to the generic definition, there is also a mapping to the wider concepts, Figure 5-7; direct mappings are between:

- Degree of Uncertainty and Chance to Occur,

- Return and Outcome - Return is the only outcome that the financial definition is concerned with.

The wider mapping is between:

- Asset and System - System has been added from the wider model to show the relationship to Asset. The concept of an asset has not been included in the generic definition in this way as it suggests ownership related to the outcome where other definitions may be concerned with 
Outcomes relating to people or property which isn't owned.

This mapping shows some interesting points regarding the financial definition of risk, firstly the sector has included the Outcome that it is concerned about in its definition. This means in terms of the generic definition that there is only ever one outcome to be considered and that is Return. Secondly the definition includes the system, this focuses the view to be taken of the system to purely financial, although this isn't a problem in the financial sector but would be for example in the railways where the safety consideration is key.

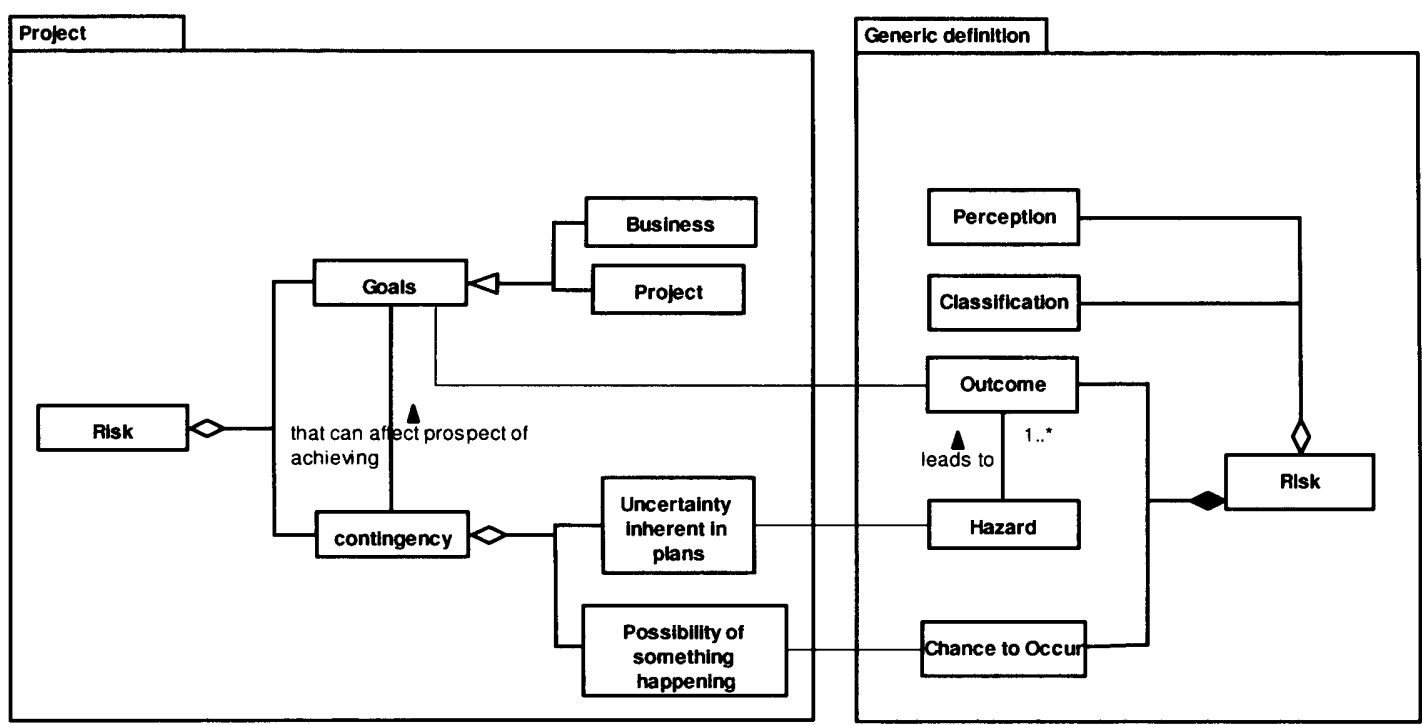

Figure 5-8 - Project

Two direct mappings are possible between the project related risk definition, taken from BS6079 (BSI 2000), and the generic, there is also one wider mapping, Figure 5-8; direct mappings are:

- Possibility of Something Happening to Chance to Occur and

- Goals to Outcome

The wider mapping is between:

- Uncertainty Inherent in Plans and Hazard - in this case Hazard has been included from the wider model to show the relationship of Uncertainty to the causal effects of risks.

The fact that hazards are included in the definition shows that there is a 
dynamic aspect to the definition, it is telling you to consider the events leading up to the outcome as part of the definition of the outcome itself. The usage of the word goal in the definition is interesting as it provides a positive view of the outcome, a desired achievement, rather than the negative view which is taken in most cases where risk is considered.

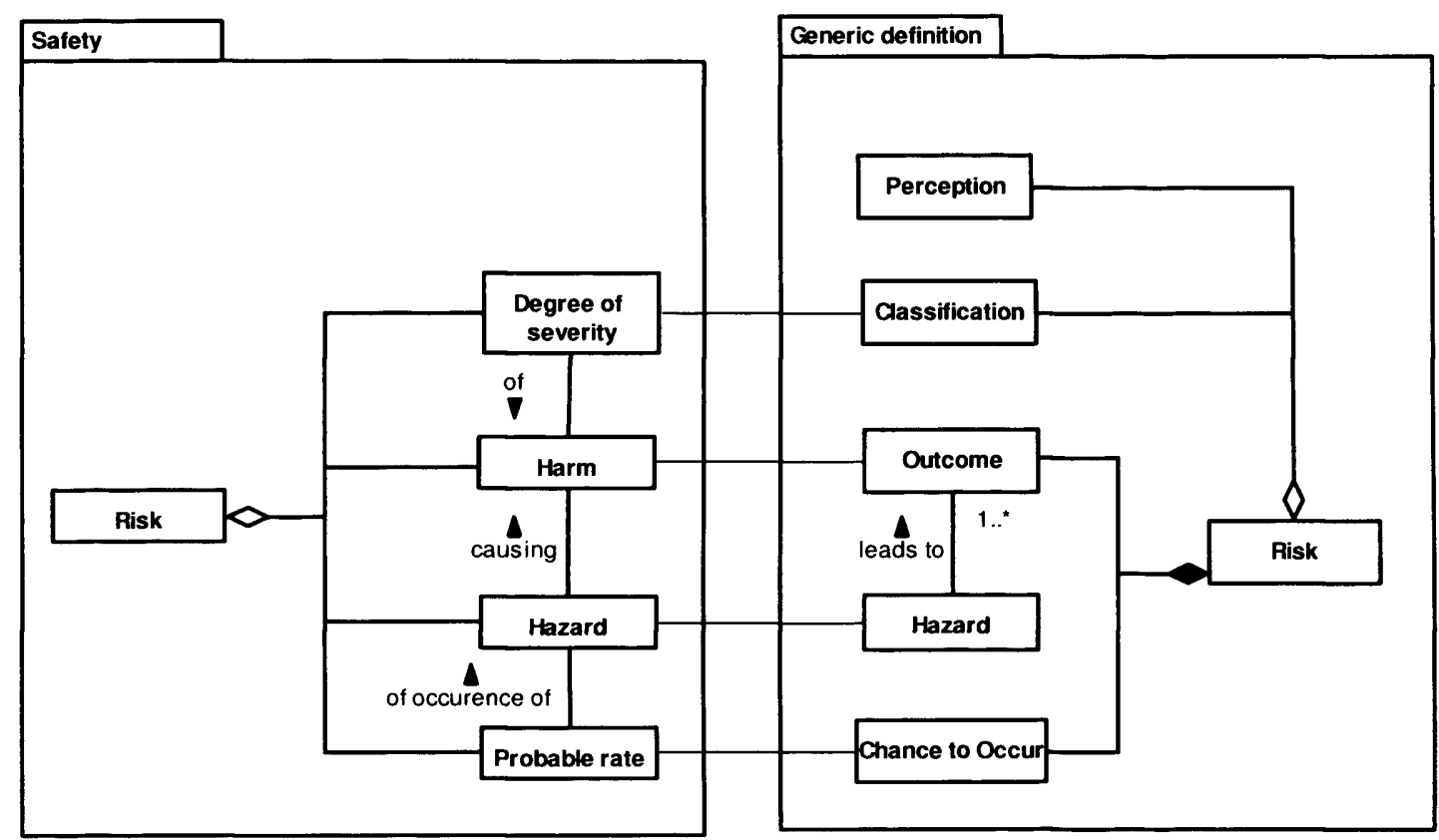

Figure 5-9 - Safety

There are three direct mappings between the safety based definition, taken from EN 50126 (CENELEC 1999), and the generic definition and one wider mapping, Figure 5-9; direct mappings are:

- Probable Rate to Chance to Occur

- Harm to Outcome and

- Degree of Severity to Classification

The wider mapping is:

- Hazard to Hazard

Similarly to the project definition hazard is incorporated here, again giving a dynamic complication to the definition. Severity which is also included provides classifications for the outcome, this has been included as a nonmandatory part of the generic definition. It is much easier to define a relevant 
set of classifications within a well established industry, however if setting out on a risk management exercise for the first time it is unlikely to add value to the initial work.

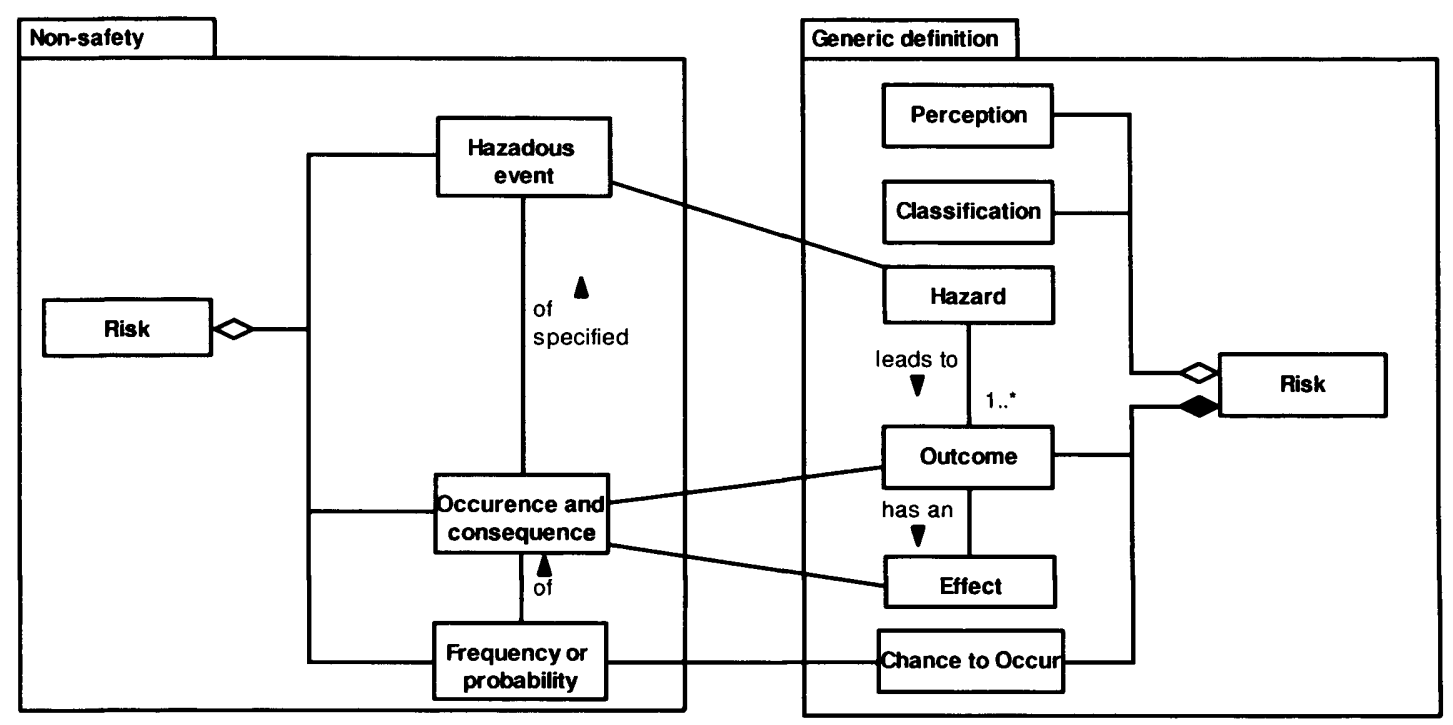

Figure 5-10 - Non-safety

There are two direct mappings and one mapping to the wider concept when considering a non-safety based risk taken from BS8444 (BSI 1996), Figure 5-10. The two direct mappings are between:

- Frequency or Probability and Chance to Occur and

- Occurrence and consequence and Outcome - this is only a partial mapping as the Non-safety definition also relates to the effect of the Outcome after it has occurred.

- Occurrence and consequence and Outcome - maps specifically to the consequence element.

The wider mapping is between:

- Hazardous Event and Hazard.

This definition considers both the hazards and the effects of the outcome which makes a risk something almost impossible to consider as it includes all possible pre and post scenarios. This definition is closer to a definition of risk analysis rather than risk. 


\subsubsection{Mapping considerations}

Many of these definitions have inherent time considerations within them, these are generally seen through the use of the term 'Hazard' or 'Consequence' which focus the reader on the pre-ceding and post outcome happenings. The definition presented here has removed this time consideration from the basic risk definition to focus the reader on the main issue the problem outcome. Obviously timing is still important and will be incorporated through relationships with the wider terminology and implementation of the associated processes.

Hazard has come up a lot in the mappings but does not play such a central role in the definition of a Risk, this work has chosen to eliminate the complexity of timing within the definition of Risk, hence the relationship to hazards which can then be investigated through causal evaluation.

There is still an unresolved issue with the term 'hazard' which may need to be replaced with a more general term which does not imply a negative. In many situations there is a level of synergy between hazard and risk which needs to be investigated further but is outside the scope of this work. 


\subsection{Risk Ontology}

Having defined the terms within risk and the relationships to surrounding terms this final diagram, Figure 5-11, relates the terms already discussed to the concepts within the processes and can be considered as a generic ontology for risk.

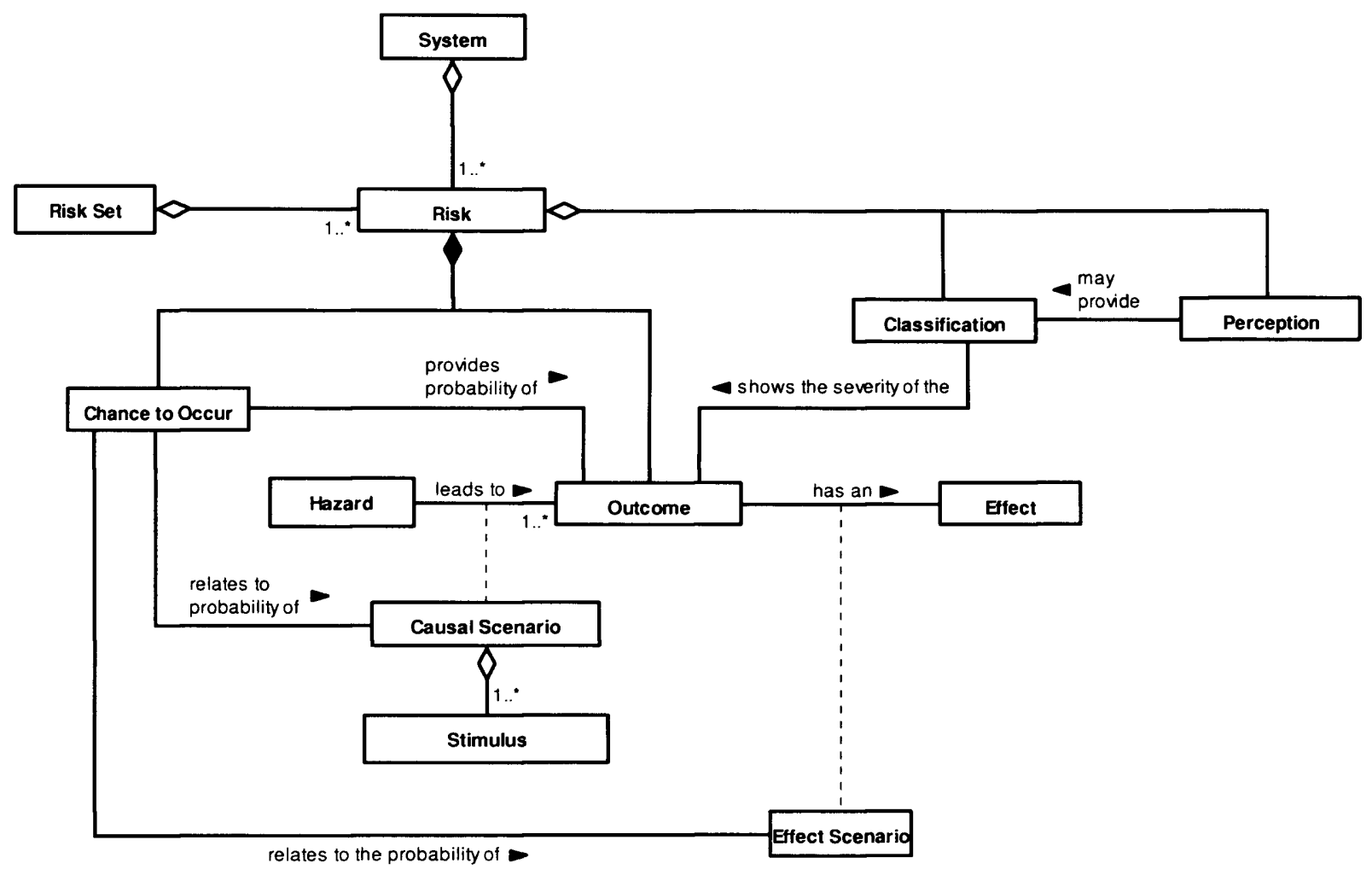

Figure 5-11 - Risk Ontology

A clear definition of terminology of risk including the relationships between the terms is imperative. Without understanding these relationships it is impossible to consistently discuss or manage risk. When compared with the Risk submodel within the OMG quality of service and fault tolerance profile for IT (OMG 2008b) discussed in Chapter 2 this provides a clear and usable set of terms and relationships which for risk for any industry or application. 


\subsection{Conclusions}

The objective of this chapter was to define risk and present an ontology for risk management.

Using the UML as a formalisation tool this chapter has presented a generic definition of risk and placed it within an ontology for risk management. The ontology provides the relationships between the definition and associated terminology.

The ontology is central to understanding relationships between other risk standards, to aid with this understanding mappings have been developed between the generic risk definition and the standards discussed in chapter 2 . From these mappings a number of salient points were highlighted including the over complication of the definition.

The chapter has also shown mappings between the definition of risk discussed here and definitions discussed in chapter 2, this has enabled further clarity and questioning of the meanings of terminology from specific industries and how they relate to each other.

Understanding the terminology and ontology of risk and risk management provides a good grounding enabling a clearer expansion across the risk domain. Mappings further support detailed industry specific approaches by enabling recognition and supporting the understanding of relationships between generic and industry specific ontologies. However, this work can not stop at terminology - without a consistent approach to the management of risk the terminology and ontology will be of use for discussion but serve no purpose in practical application.

This work will continue by providing a formalisation of the risk management approach and methodology using the UML and the seven views approach. 


\section{Processes}

\subsection{Introduction}

Chapter 5 presented an ontology for risk, formatted through the use of the $\mathrm{UML}$, presenting the terminology of risk management. It highlighted that this terminology although useful in its own right can not be used without processes to realise it.

The purpose of this chapter is to define the processes required to carry out risk management using a multi-view approach. This will be achieved by using the 'Seven Views Approach', discussed in chapter 4, to define the processes, behaviours, information and methodology for risk management. The chapter presents the six risk management processes defined by this work. After defining the processes a guide is given as to the expected outputs of the processes.

\subsection{Process Formalisation}

This section presents the Requirements View, Process Content View and Information View giving an overview of the all of the processes, it also presents the Process Behaviour View for three of the processes; that of Concern Identification, Risk definition and Evaluation. It is expected that these processes will be implementable within the framework proposed by the draft ISO 31000 Risk management - Principles and guidelines on implementation. 


\subsection{Requirements View - Risk Management Requirements}

The Requirements View provides an understanding of the things that need to be done to manage risk and the things that need to be understood.

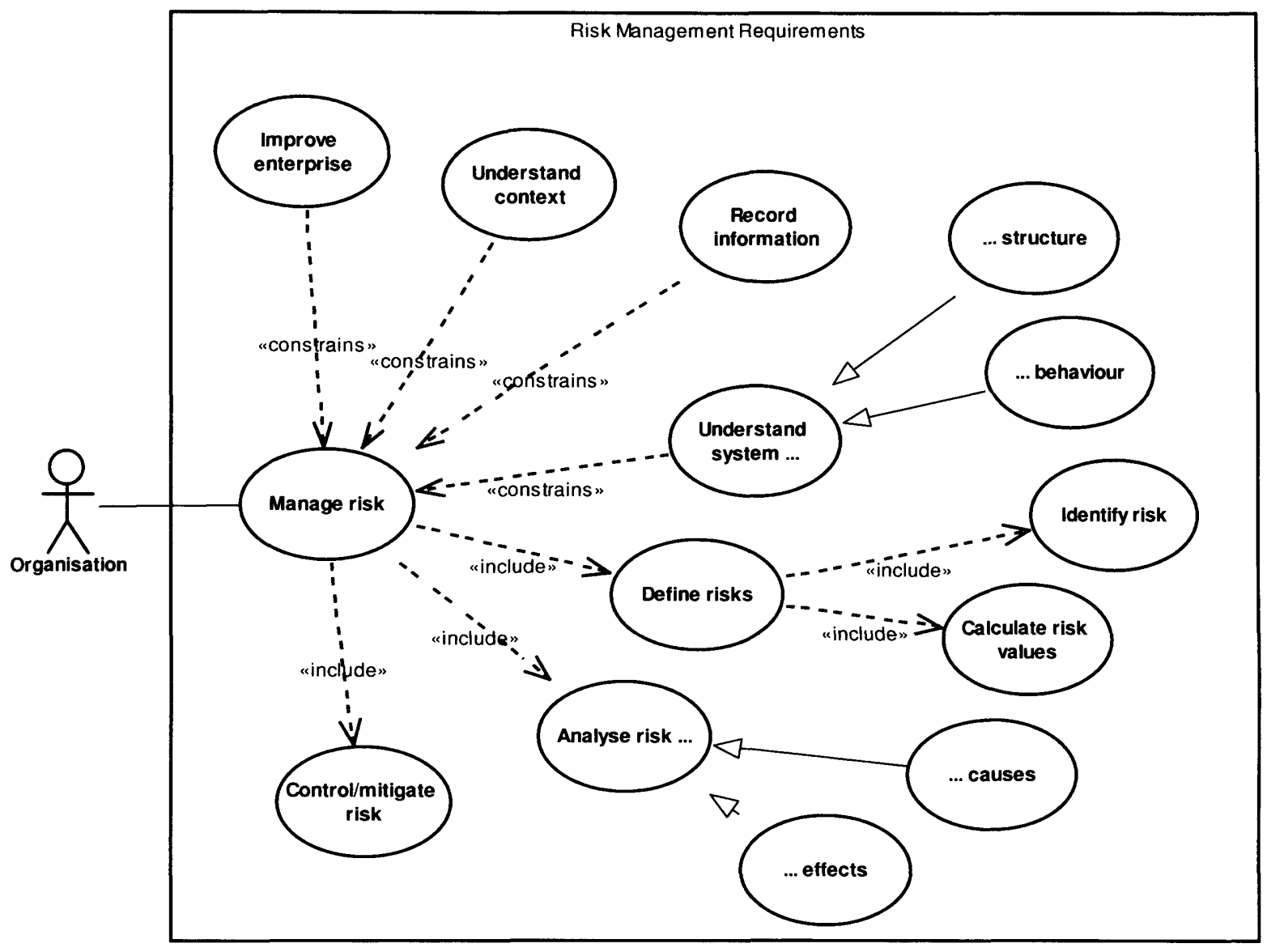

Figure 6-1 - Risk Management Requirements

Once it has been stated it is obvious that defining, analysing and controlling risk are things that must be completed to manage risk. However, the recording of such a fundamental concept of 'why this is being carried out' can often be missed BPMN (OMG 2008a) for example does not provide the ability to record why a process is being carried out. It is also easy to miss or forget those things which need to be understood; it is the act of recording them which provides focus.

The diagram above (Figure 6-1) provides a view of the reason for carrying out Risk Management in this case it is to improve an enterprise. In other situations it may be for health and safety, project planning or financial reasons. With an understanding of why risk management is being carried out the context in which it is being applied must also be considered. The context 
will ensure that the risk management has the right focus e.g. the risks to be considered are mostly related to people, technical or marketing questions.

\subsection{Process Content View - Risk Processes}

The Process content view provides a static representation of the processes showing the activities to be carried out and the artefacts to be produced or consumed.

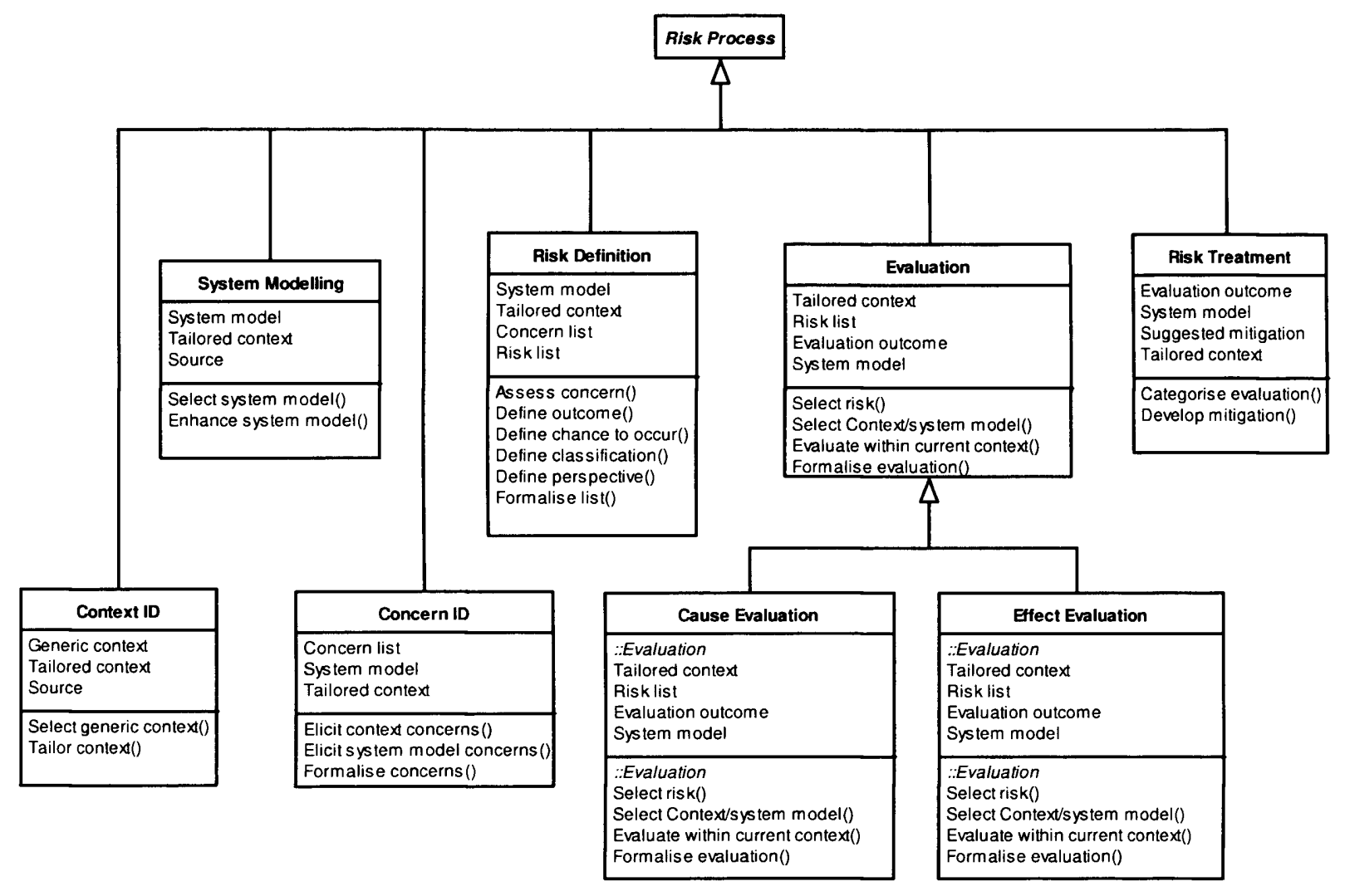

Figure 6-2 - Risk Processes

This approach to the definition of risk management processes has provided a set of processes, Figure 6-2, rather than the single process provided in most standards. This use of multiple processes each used as and when required enables a more flexible and realistic approach to risk management. It means that one process, Concern ID for example, could be used multiple times whilst the Risk Definition process may only used once.

A brief overview of each process is given here before the behavioural view for each process and overall information view are presented. 
Context ID:-This presents and discusses the contexts, the needs and scope, associated with an entity such as a company. These contexts provide the different points of view from which risk can be understood. They also show the relationships between different contexts.

System Modelling:-This retrieves or provides the relevant system models, these may be static or behavioural. The system models must relate to the relevant contexts.

Concern ID:-This process provides hazard, risks, causes, outcomes or worries based on context and system models. The use of context and system models to identify concerns provides a repeatable approach without relying on tacit knowledge.

Risk Definition:-This process is used to provide as much detail regarding a risk as possible this may include applying assessment techniques providing knowledge and detail of the risks.

Evaluation:-The Evaluation process applies a risk to the contexts being investigated this will provide an understanding of the risk on the system or the system on the risk.

Cause Evaluation:-The Cause evaluation process applies a risk to the contexts being investigated to provide an understanding of the effect of the system on the risk. i.e. it is looking backwards or into the system to see what in the system will cause or affect the outcome before it has happened.

Effect Evaluation:-The Effect evaluation process applies a risk to the contexts being investigated providing an understanding of the effect of the risk on the system. i.e. it is looking forward to see what happens in the system after the outcome has occurred.

Risk Treatment:-This process enables the decision as to whether any mitigation is necessary or to be considered. Once this has been agreed it provides possible mitigations for evaluation. 
Having defined the purpose of each process the behaviour also needs to be defined showing who the process is achieved.

\subsubsection{Context Identification Process}

This presents and discusses the contexts associated with any entity. These contexts provide the different points of view from which risk can be understood. They also show the relationships between different contexts.

There are a number of points to remember when defining contexts firstly, and this goes for all of the processes, they don't have to be finished at the end of the first run through the process - the process can and should be run again to add detail to the information. In addition to ensure that the contexts do not become over complicated it is worth applying Miller's magic number (Miller 1956) for both the number of contexts and the needs defined within.

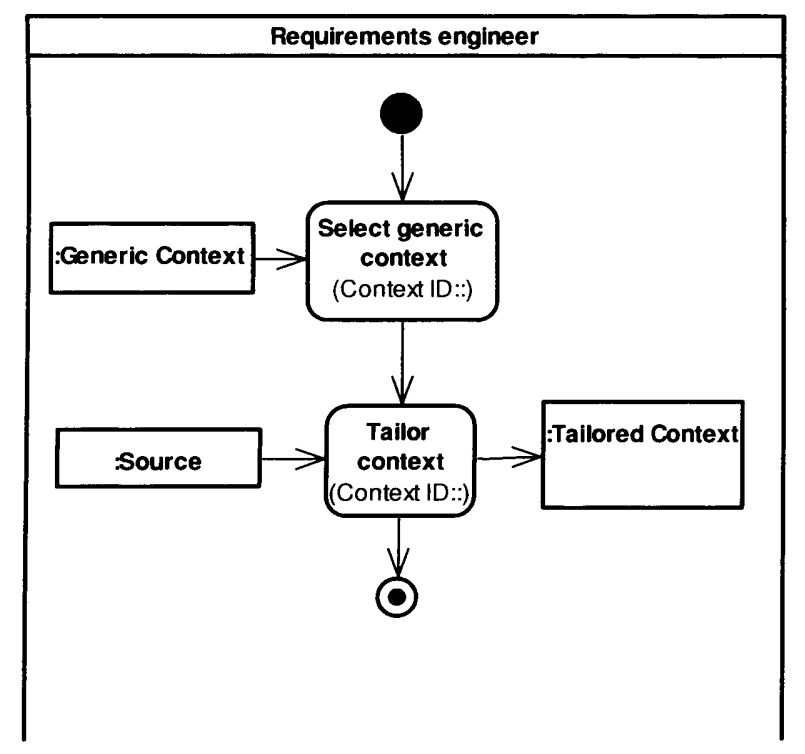

Figure 6-3 - Context ID

- Select generic context - Selecting Generic contexts may be achieved through the review of context libraries. In the case where sufficient knowledge of the domain exists this activity may create a list of the context names to be fully defined later.

- Tailor context - Tailoring a context defines the boundary, stakeholders and internal needs of a viewpoint. This tailoring relies on the ability to focus on 
the development of needs from a specific point of view. In the situation where contexts already exist this activity may only be a check to see that the contexts are still applicable. Where there is in-sufficient domain knowledge it is preferable to define a simple set of needs to be improved later when more information will be available.

\subsubsection{System Modelling Process}

This retrieves or provides the relevant system models, these may be static or behavioural. The system models must relate to the relevant contexts.

This process has not been defined to re-develop any system models that may exist, but to ensure that the benefit of re-use is gained in as many areas as possible existing models should be used and further developed where they do not provide sufficient detail in the area of focus.

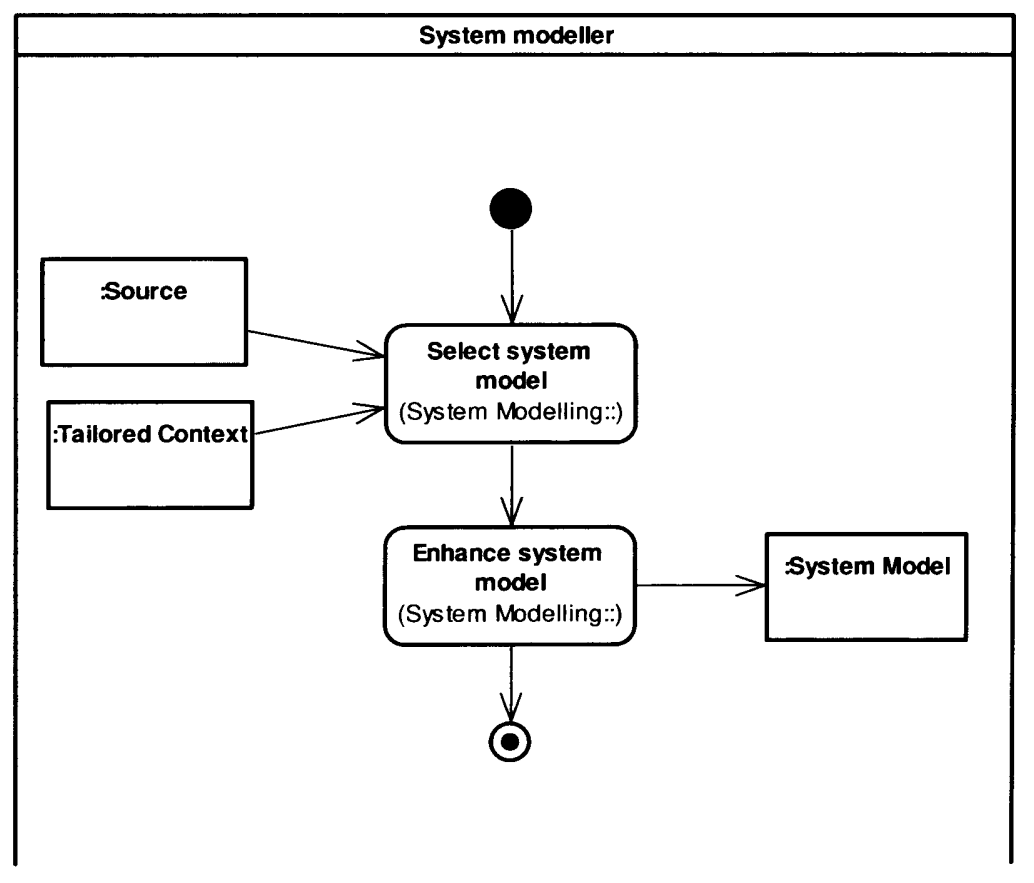

Figure 6-4 - System Modelling

- Select system model - The activity identifies the relevant models from the Source for specific Tailored Contexts. This activity is based on the premise that a system model will already exist for the defined contexts, for a system it may be the architecture or behaviour, for a company it could be 
the enterprise architecture (EA) or process model.

- Enhance system model - This activity ensures that the selected System Models match the boundaries, stakeholders and needs of the Tailored Contexts. This may involve changes to the models to ensure consistency. Notes should be made as to why changes have been made and record any issues which may affect the Source.

\subsubsection{Concern Identification Process}

This process provides hazard, risks, causes, outcomes or worries based on the Contexts and systems models.

A number of external factors are also generally considered here, they tend to include environment, Health \& Safety, Human Factors, etc. if these have not been captured in a context or as external relationships on the system model. It is always important to capture the source of the concern so that more information can be obtained to ensure that full consideration is given when carrying out the risk definition process.

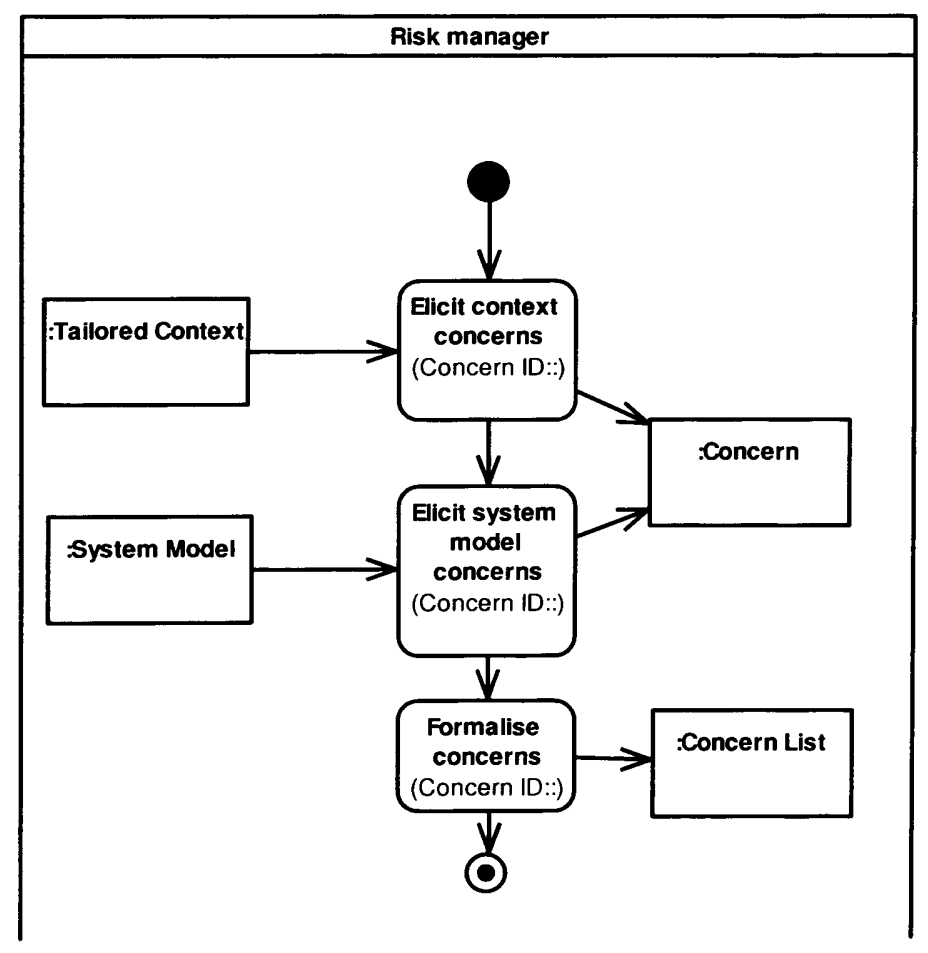

Figure 6-5 - Concern ID 
- Elicit context concerns - During this activity any concerns based on the Tailored Contexts are identified, the Concerns may be linked to conflicting, missing, additional or unfulfilable needs. At this point a concern need not be a risk or a problem it can be any question that someone wants to ask about the system and although it would be preferred that it is based on the Tailored context or System Model this isn't a pre-requisite, other questions may in-turn provide improvements to the Tailored contexts or System Models.

- Elicit system model concerns - During this activity any concerns based on the System Models are identified, the concerns may be linked to parts of the system, communications within the system or the behaviour of the system. This could be as detailed as to question every relationship, attribute and behaviour.

- Formalise concerns - During this activity the concerns identified within the other activities will be recorded formally, this means that they must be named, numbered, referenced and described.

\subsubsection{Risk Definition Process}

The Risk Definition process is used to define risks and provide as much detail regarding each risk as possible this may include applying assessment techniques providing knowledge and detail of the risk.

It is important to note that this process has been defined to look at the risk from a static point of view, the inclusion of dynamics will be considered when carrying out the evaluation process. 


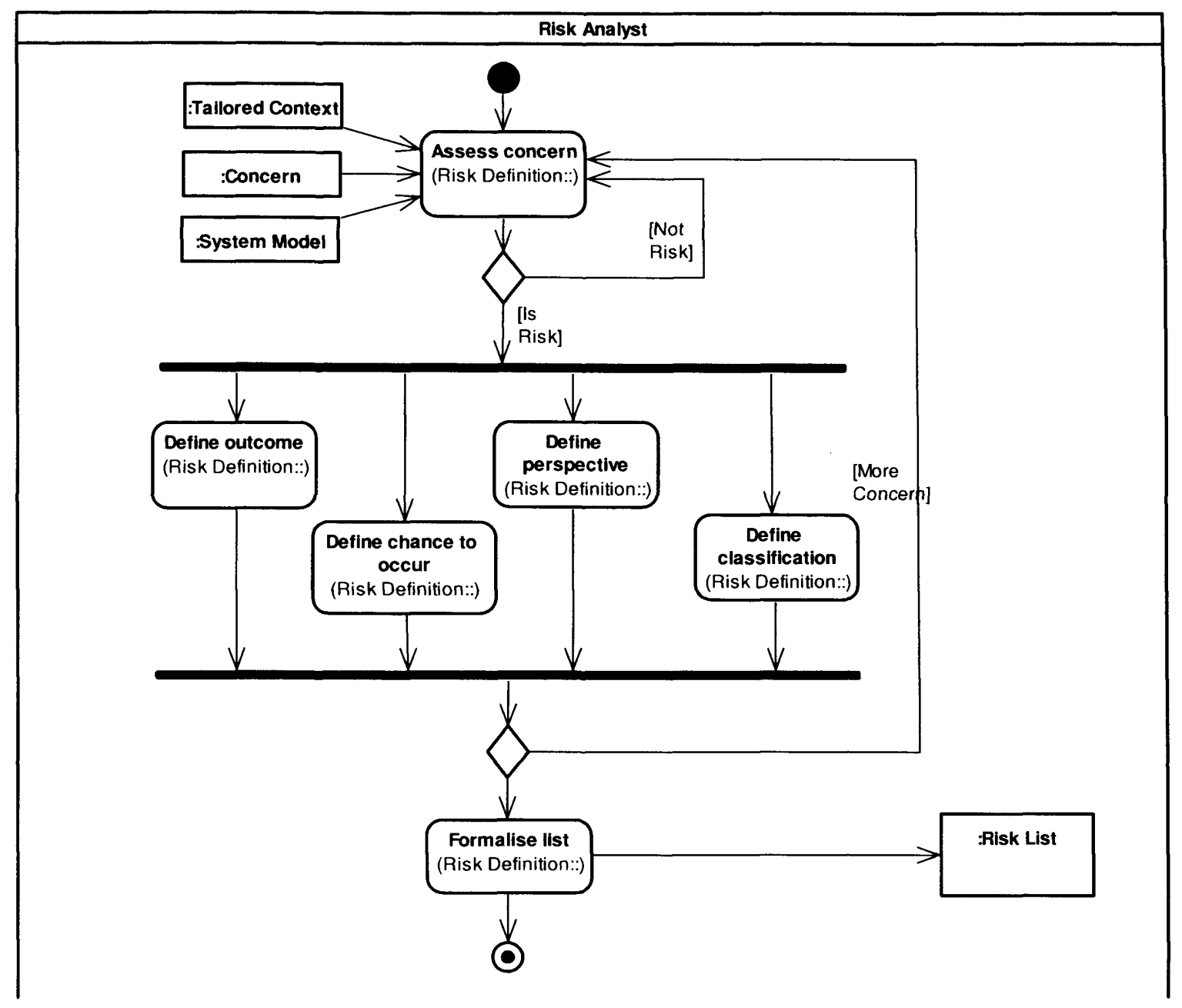

Figure 6-6 - Risk Definition

- Assess concern - During this activity each concern is reviewed and categorised. The main category of interest is Risk i.e. this concern can be considered to be a Risk. Other categories may be Hazard or Effect these could be used to signify the need to consider these concerns when carrying out the evaluations. A concern will be selected as a risk if it can be shown to be one of the main areas for concern. It would be nice to make this more scientific however this doesn't seem possible at the moment due to the wide variety of systems and organisations to which these processes may be applied.

- Define outcome - During this activity the outcome associated with the Risk will be identified and recorded. This may required the use of a tool or technique aimed at the identification of outcomes.

- Define chance to occur - During this activity the Chance to Occur of the 
Outcome will be calculated and recorded. This is likely to require the use of an assessment technique aimed at the definition of probability, it may be possible to choose an assessment technique from those referenced in Chapter 2. In some cases the Chance to Occur will be very difficult or expensive to calculate, in these cases there is a tendency to let the classification or perception lead rather than having a full definition. This in itself can be dangerous, however the values will depend on the assessment technique employed.

- Define classification - During this activity the classifications of the Risk will be identified and recorded. Classification are discussed in the generic definition of risk in chapter 2 however, specific classifications relevant to the situation may need to be developed. The classifications are defined as part of the generic risk terminology.

- Define perspective - During this activity the perspective of different stakeholders related to the Risk will be identified and recorded. Understanding the perspective of a stakeholder or stakeholder group is a subject in its own right and has been discussed by many including Belzer (2001) in his paper on grin and bear it practices in risk management and alluded to by Clarke (2007) when discussing probabilistic and possibilistic risk.

- Formalise list - During this activity the information defined relevant to each risk will be recorded formally, this means that as much of the information as possible is complete and at a minimum each risk must be named, numbered, described and have an Outcome and Chance to Occur.

\subsubsection{Evaluation Process}

The Evaluation process applies a risk to the contexts under investigation providing an understanding of the risk on the system or the system on the risk.

This process has been defined with two specific types in mind, causal evaluation and effect evaluation. Causal evaluation provides consideration of all the events that occur leading up to the occurrence of the outcome. Effect 
evaluation provides the ability to consider the events that occur after the outcome, this may include exit procedures, notification of emergency services and clean up.

As the process for each, cause and effect, is the same other than the focus of pre-ceding or following events the overall process of evaluation will be described.

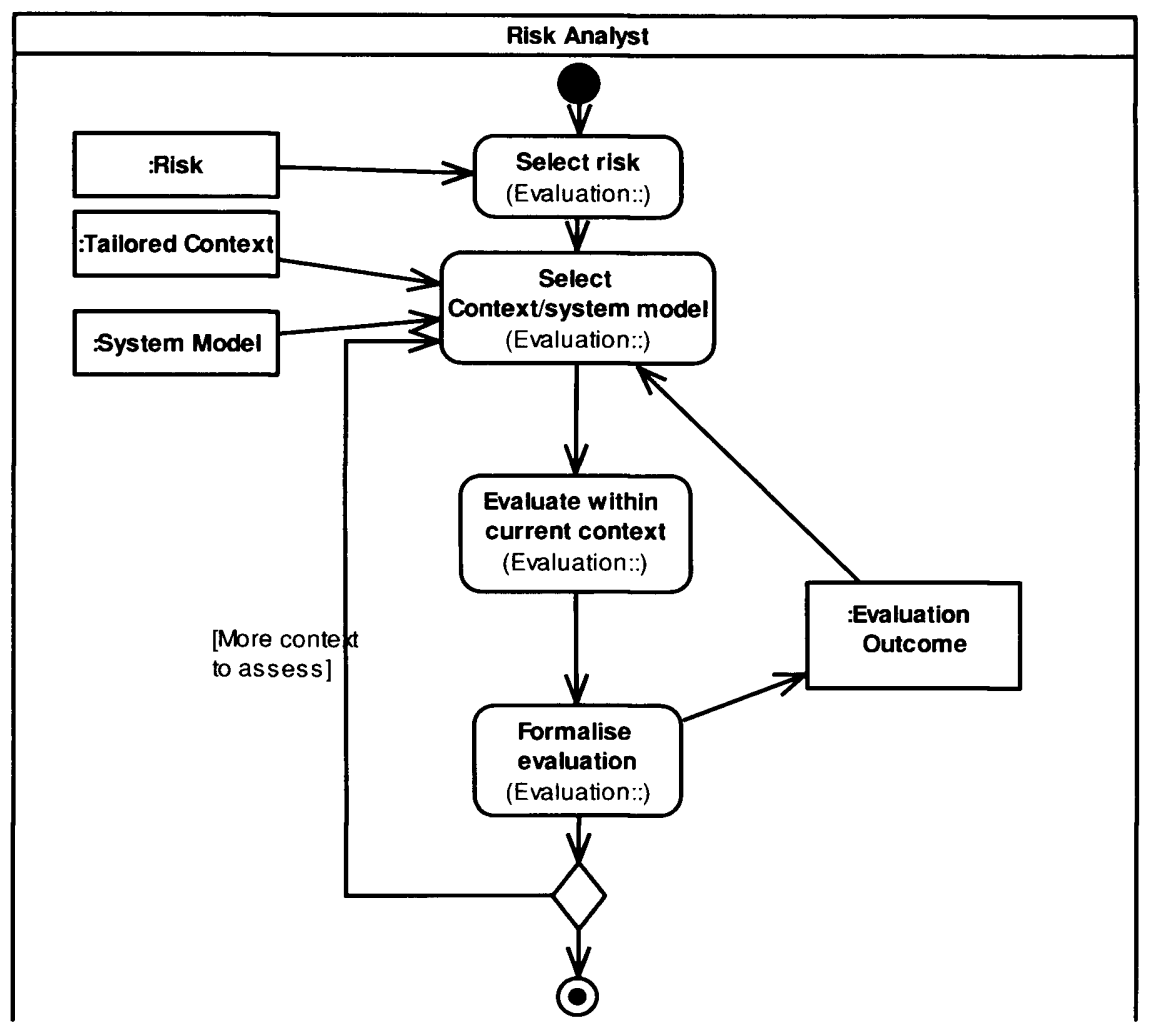

Figure 6-7 - Evaluation

- Select risk - This activity chooses the Risk to be evaluated from the Risk List.

- Select Context/system model - Selects a context and relevant system model within which the Risk will be evaluated. This is to ensure that the relevant evaluation is carried out, there is no point in carrying out a financial evaluation for a technical Risk before the technical evaluation has been carried out.

- Evaluate within current context - This activity applies the risk to relevant scenarios from the context to the system model. This will be carried out in 
conjunction with the use of any specific techniques identified. The result of the activity will be an increased understanding of the relationship between the risk and the system. This activity is defined as 'current context' as once evaluated for one context it is possible to follow the relationships between contexts and apply the Evaluation Outcome to the next context. This also provides an approach to effect analysis.

- Formalise evaluation - This activity ensures that a full record of the effect of a risk on a context has been kept.

Due to the use of tailoring applied to this process the cause and effect evaluation processes have not been described individually an example of each analysis is shown in section 6.7. The relationship between cause and effect is also investigated by Restrepo et al (2008) whilst understanding how different causes of accidents are associated with consequence measures.

\subsubsection{Risk Treatment Process}

This process enables the decision as to whether any mitigation is to be considered. If it is agreed that mitigation is required then the process provides Suggested Mitigations for evaluation.

It is important to remember that the development of mitigations may be projects or programmes in their own right, this process is designed to be a place holder to ensure that the relevant projects are developed in response to the risks and their evaluation. However, this does not mean that all project developments should relate directly back to risks or their treatment.

Risk Treatment in this manner could be considered to be the art of System Engineering in practice. Using the information gathered and derived through all of the other processes as a basis will provide all of the inputs to a standard Systems Engineering approach. It is important to ensure that treatments aren't defined for there own sake and remember that some risks can not be removed. 


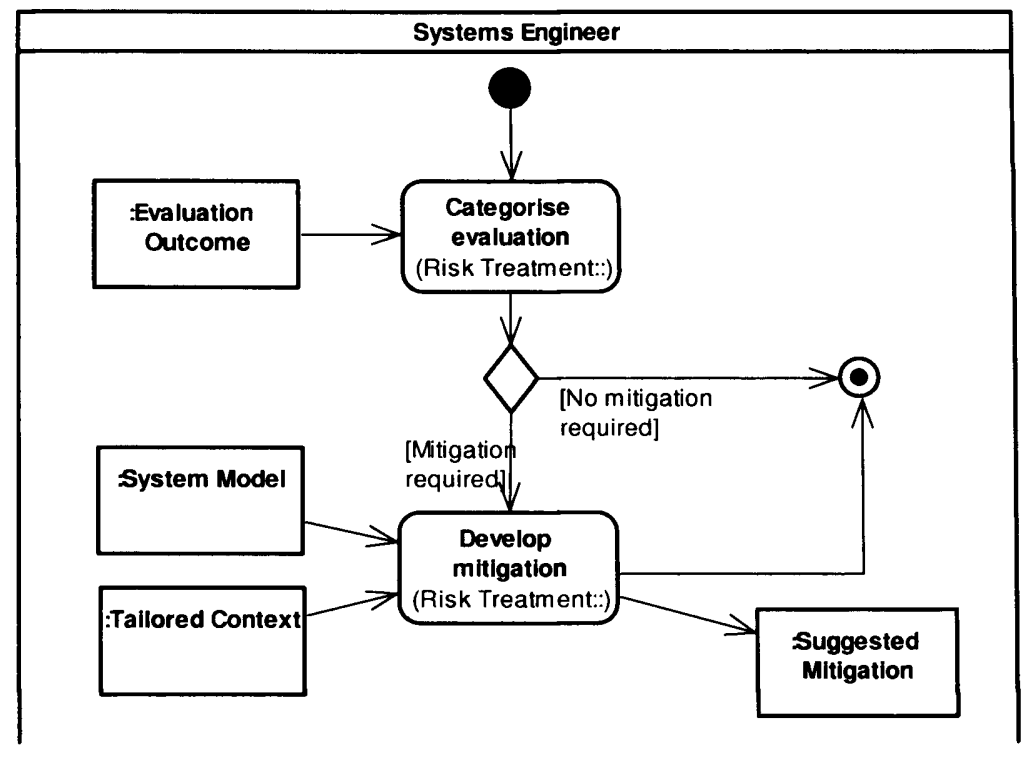

Figure 6-8 - Risk Treatment

- Categorise evaluation - This enables the evaluation to be assessed effectively deciding whether the Risk is undesirable enough to require action to stop it occurring or reduce the effect.

- Develop mitigation - This activity allows for the development of mitigations to the risk, mitigations are changes to the current system that reduce or remove causes and effects of the Risk. The development of a mitigation may require anything from 5 seconds thought to a full system development, in some cases this could mean the creation of a new organisation. 


\subsection{Information View - Risk Processes Artefacts}

The processes described above have detailed activities to be carried out, they have also defined information and artefacts that will be used and developed within the management of risk. This information needs to be drawn together to ensure that it will be used and is not just adding red tape.

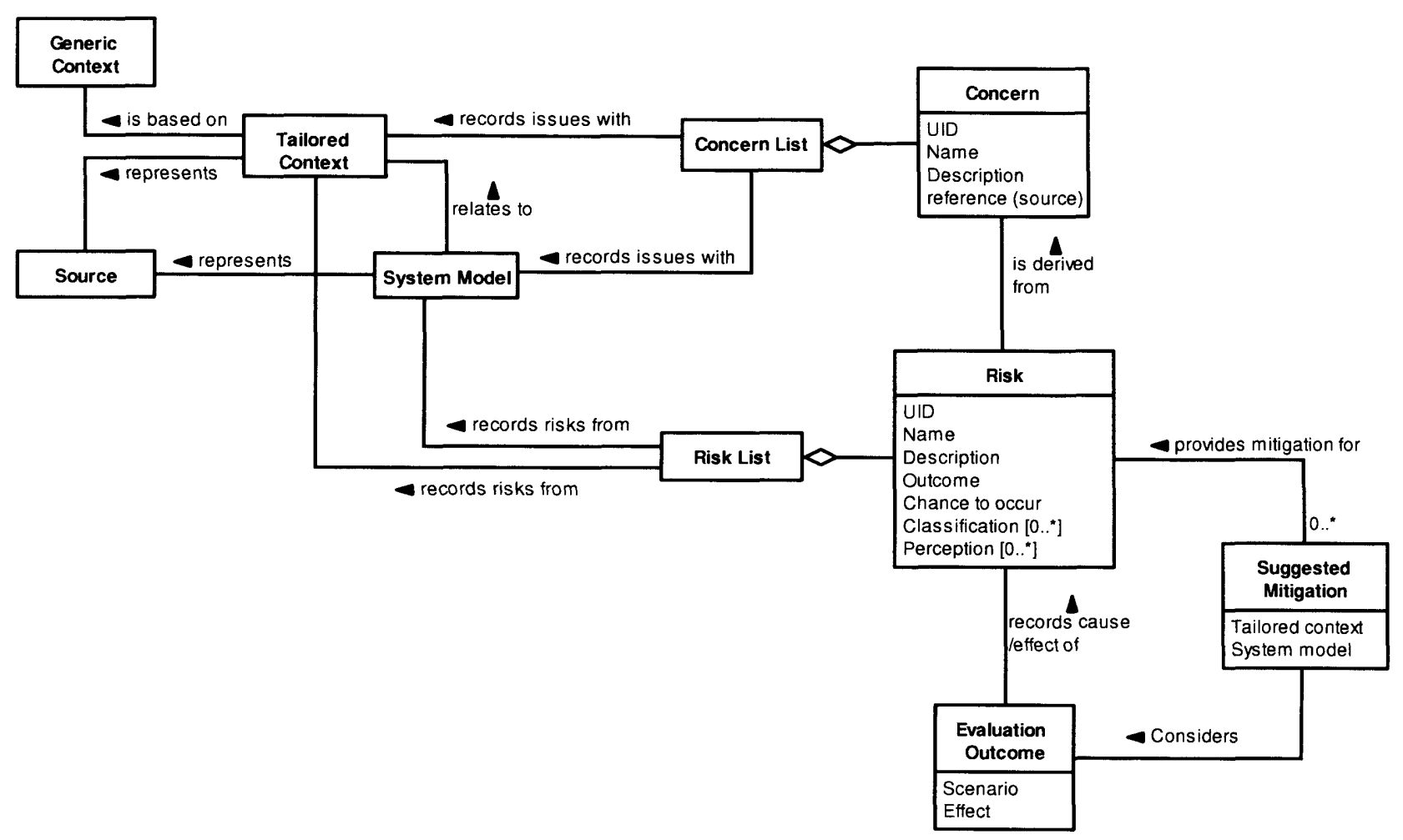

Figure 6-9 - Process Artefacts

All of the Artefacts from the processes, Figure 6-9, must be related together to give an understanding of the reason for any one Artefacts existence, this could also go on to show external relationships where a document may exist for an audit or delivery purpose.

It is clear that the contexts and system models are related to understanding the source information, it is hoped that in many cases that the relevant source information already exists to maximise on re-use and remove redundancy in the duplication of existing models. Highlighting the fact that a concern and a risk are not the same thing is the difference in the detail required to understand each. A Concern is something that has a little information associated, it can be named and described but not much more where as a Risk has much more understanding and information associated with it. 
It is worth noting here that in comparison with the standard processes discussed in Chapter 2 there is a lack of communications and monitoring Artefacts. These artefacts have been omitted as it is expected that these will be part of an organisations standard process set and as such to re-define them in risk management would create redundancy within the organisations processes. If it is desirable to monitor the Risk to verify that it is still accurate then the Risk Definition Process should be executed at defined intervals for the specific risk or the whole list as required.

\subsection{Process Instance View - Methodology}

It is expected that for any set of processes there is a prescribed order of application. However, one of the main advantages of using object oriented process definition is that the processes can be used in many different orders providing flexibility to an organisation. This section describes two ways of applying the processes the first shows the theoretical ideal, as if starting from nothing, the second shows a more pragmatic approach accepting the fact that some information will already exist and that if there were no concerns then it is unlikely that the organisation would be carrying out risk management.

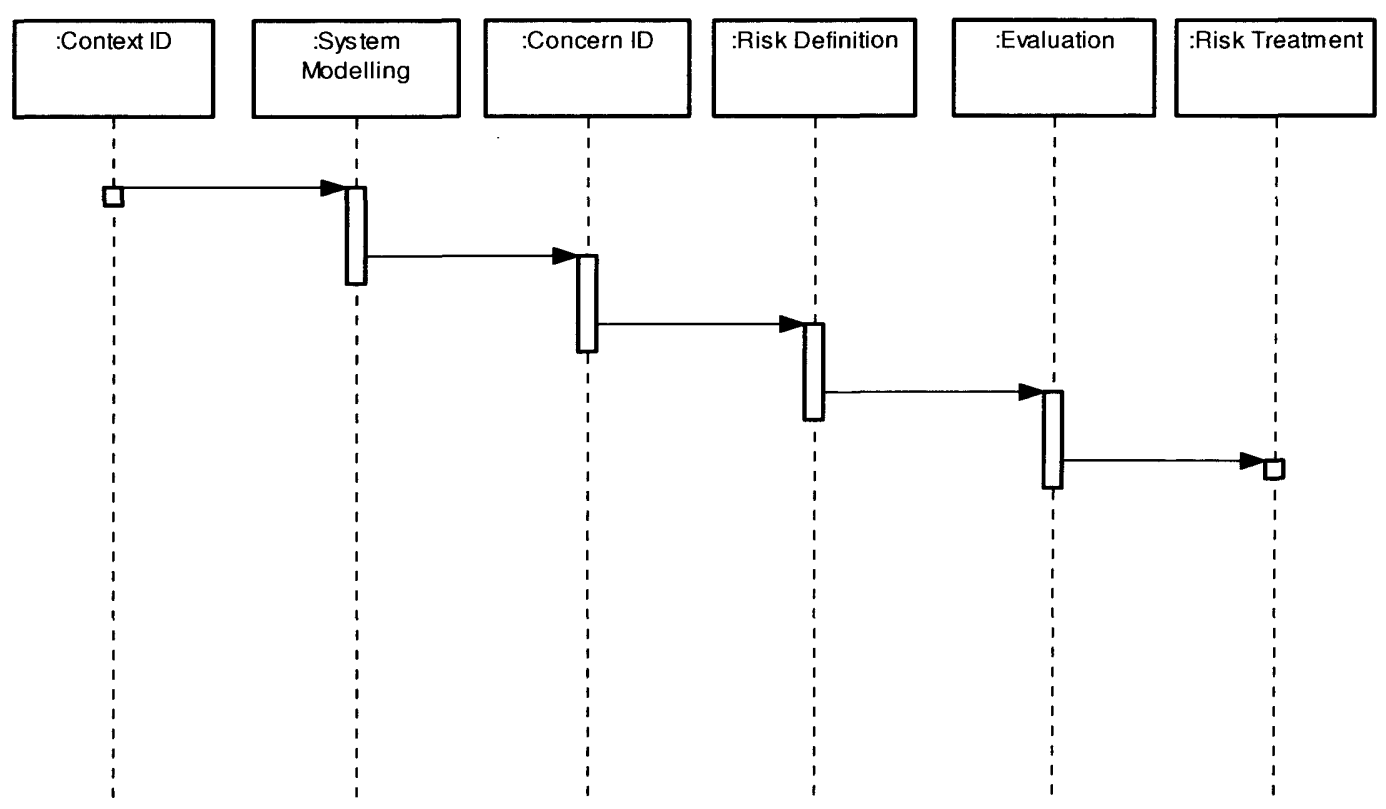

Figure 6-10 - Theoretical ideal 
When considering the theoretical ideal the first step would be to ensure that the scope of the work and the areas to be investigated were understood, this would move on to ensuring a consistent view of the system as it stands. With a full understanding of the scope of work and the system in place concerns with the system could be identified before specific risks defined. An evaluation of the risks would be carried out with a view to defining mitigations for them. This waterfall type approach, Figure $6-10$, is presented by many as the approach to projects and life cycles, it has also been shown to work well on small well defined projects but not on large multidisciplinary undertakings, Royce (1970) defines the waterfall approach before saying that it is 'risky and invites failure'. For larger projects (or organisations) a more flexible and cyclical approach is needed.

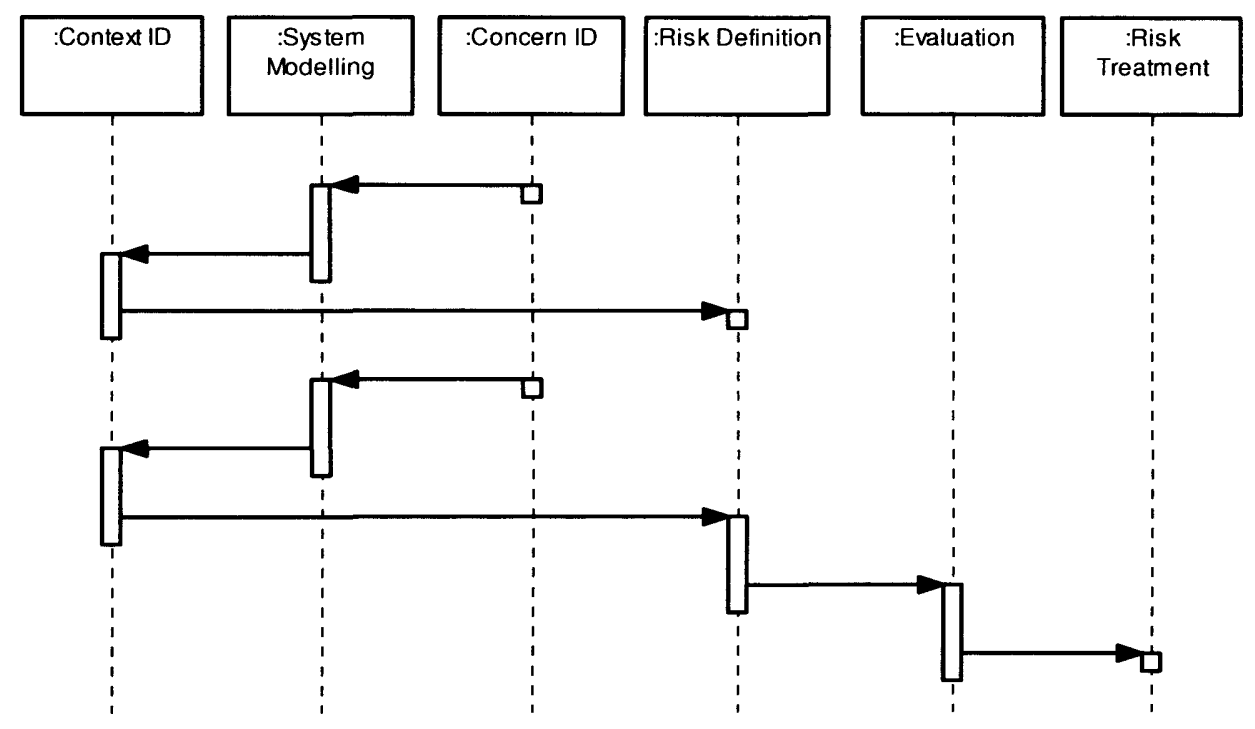

Figure 6-11 - Pragmatic approach

An example of a more pragmatic approach, Figure 6-11, would be to start by understanding the concerns first and based on these develop or retrieve the relevant sections of the system understanding. The contexts can then be developed with the knowledge of the areas to be considered before fully defining the risks. All of this work could be carried out multiple times before any evaluation is carried out or treatment defined. 


\subsection{Process Support}

The processes defined above detail the activities and artefacts required to carry out risk management. This section provides further guidance in the application of the ID Context, Risk Definition and Risk Evaluation processes.

\subsubsection{Risk Contexts}

It is well known that different types of risk exist in any organisation or entity. This is re-enforced by concepts such as balanced scorecard (Kaplan and Norton 1992) which identifies the need to consider the effects of Finance, Learning and Growth and Customer and Business Processes on the vision and strategy of the company. Zachman (1997) discusses different views within an Enterprise architecture framework looking at Data, Function, People, Time and Motivation; each of these he applies at different abstractions when looking at an enterprise. These views, whether based on Zachman or on balanced scorecard, are known as contexts.

The manner in which we are considering complex contexts and interactions can be equated to Senge (2006) discussing the systems issue of dynamic complexity; the whole being greater than the sum of the parts.

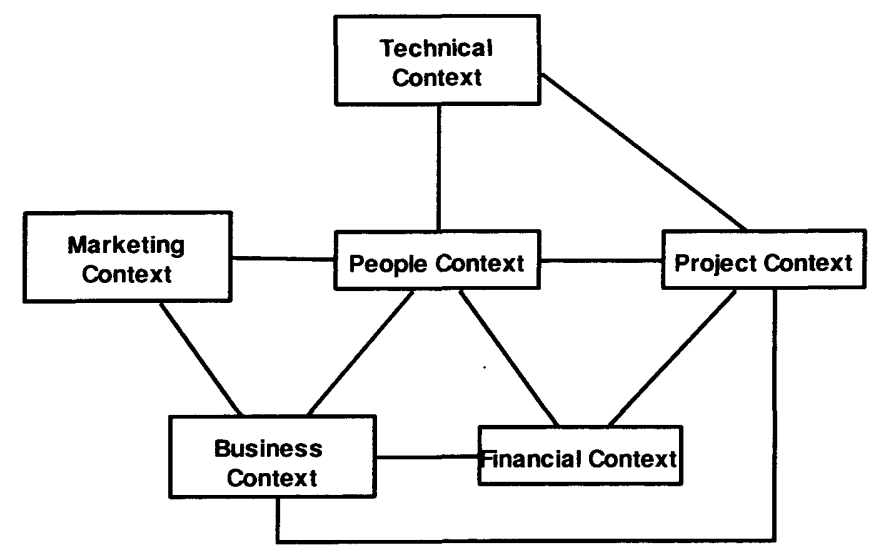

Figure 6-12 - Risk Context relationships

This set of six generic contexts and their interactions, Figure 6-12, provide a high level set of contexts, these can be considered as a starting point for identifying relevant contexts for risk management. These six contexts are Business, Finance, Marketing, People, Project and Technical.

When using the UML as a tool to aid understanding of contexts Use Case 
diagrams are used. These describe four main concepts:

- the Use Cases themselves which together describe the behaviour or desired achievement of the context,

- the System Boundary which encapsulates the Use Cases and therefore defines what is inside/outside the context,

- the Communications which represent the interactions of the context with the outside world and

- the Actors which are the things or people to which the communications connect (in this case these will always be other contexts).

All six Use Case diagrams will now be presented, starting with the Business context;

\section{Business context}

The Business context provides the overall mission of the company and the ways it believes it can achieve that mission

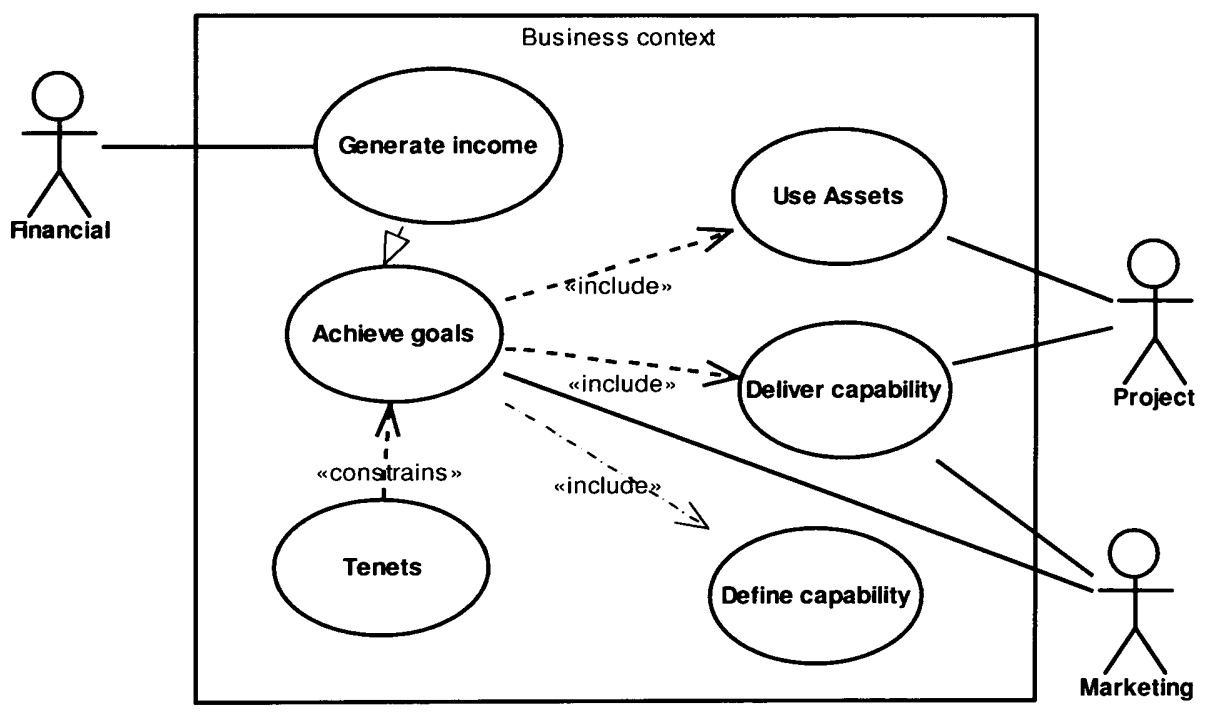

Figure 6-13 - Business Context

The overall aim of this business is to Achieve goals within the realms of its tenets and it does this by defining capabilities, delivering capabilities, and using assets. Therefore the Financial context is interested in Generating income, the Project context is interested in using assets, the Project and Marketing contexts are interested in delivering capability and the Marketing 
context is also interested in Achieving goals.

\section{Financial context}

The Financial context deals with all things relating to the companies money.

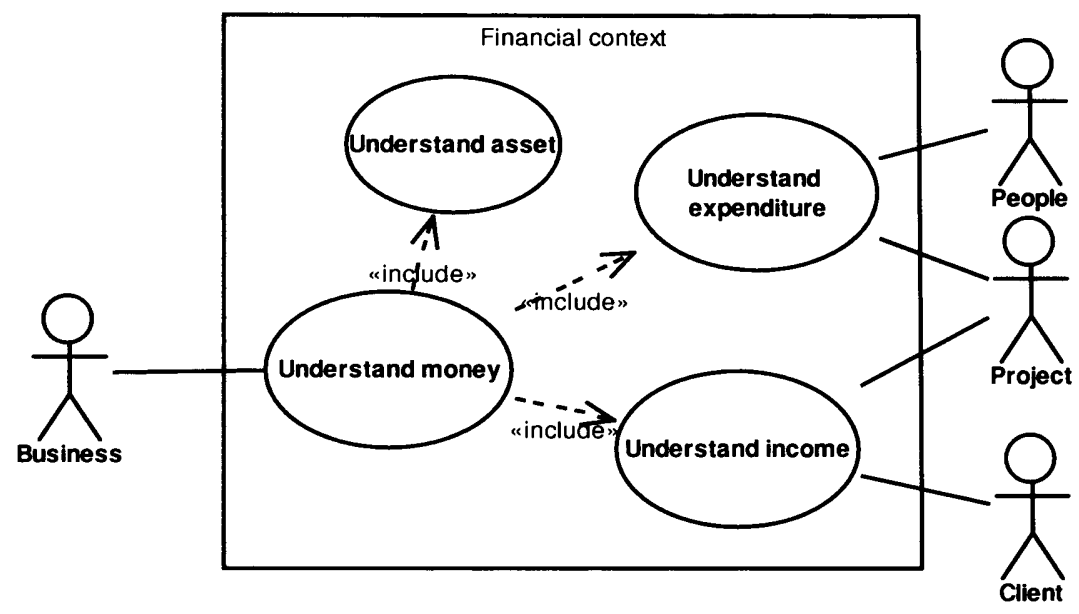

Figure 6-14 - Financial Context

The overall aim of finance is to understand money which includes understanding assets, understanding expenditure and understanding income. Understand money relates to the business context as this is the only way in which the business can work out if it is profitable or not, understand expenditure relates to the People context and understand income to the Project context. Understand income also relates to the Client 


\section{Marketing context}

The Marketing context considers the scope for sales including ways to improve the perception of the company and sales within the market.

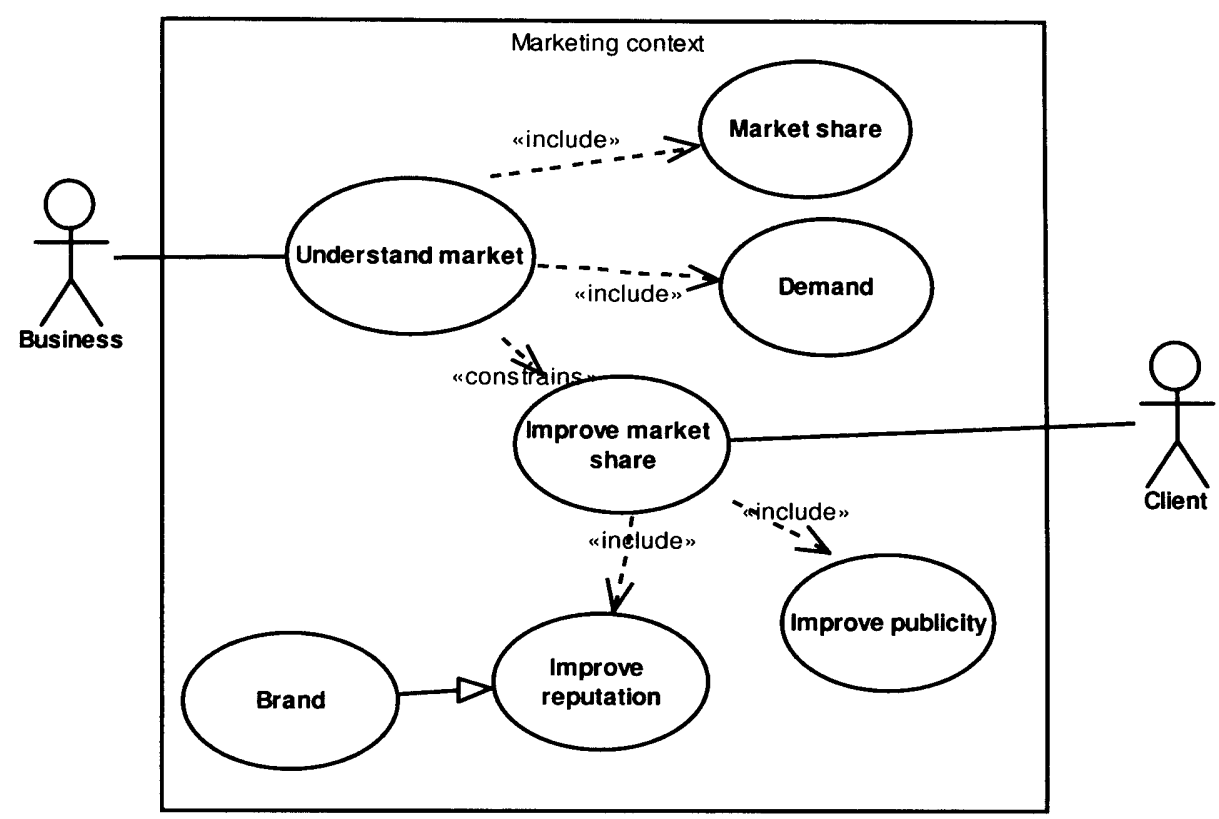

Figure 6-15 - Marketing Context

Understanding the market includes understanding market share and market demand, it also constrains the improvement of market share. Improving the market share can be achieved by improving publicity and improving reputation. "Brand" is a specific way of considering reputation and can be considered separately. The Business context is interested in understanding the market whilst the Client will be affected by improvement of market share. 


\section{Technical Context}

The Technical context is concerned with the product for its full lifecycle i.e. from concept through to disposal.

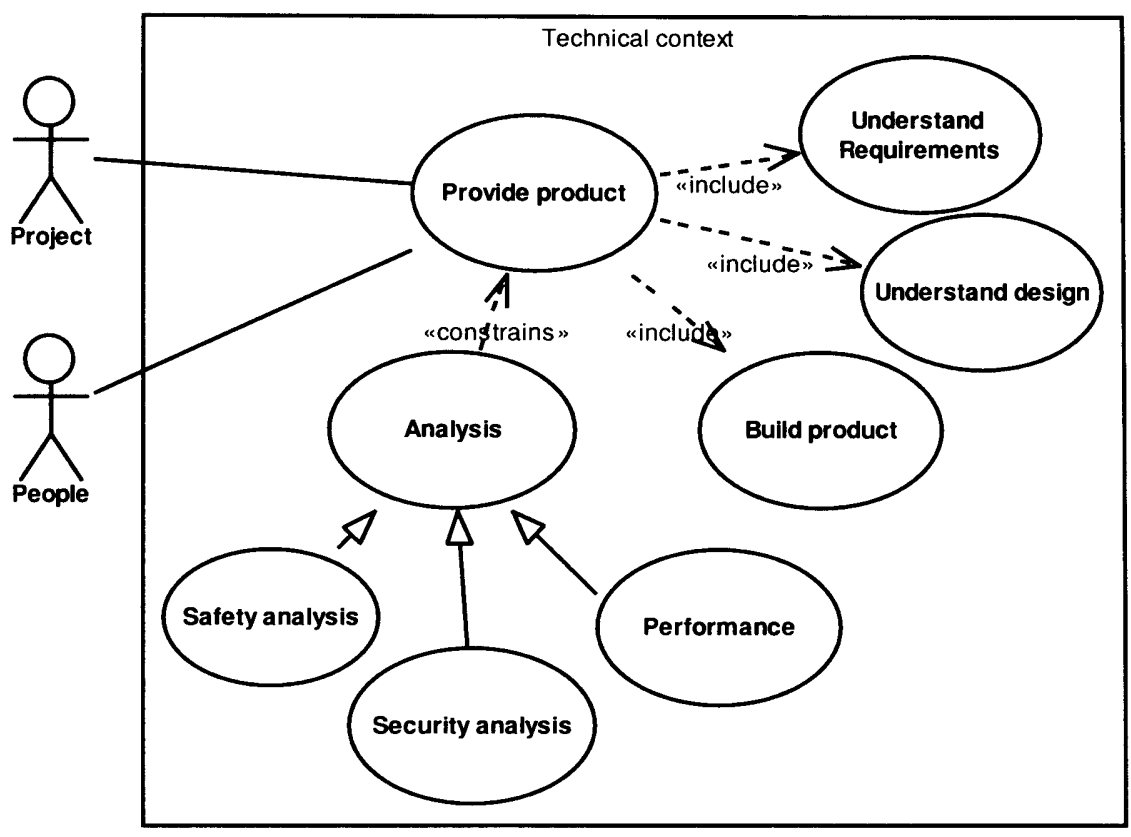

Figure 6-16 - Technical Context

The Technical context is focused on providing products. To provide products the company must understand the requirements, develop a design and build the product. Within each of these areas analysis will be performed and this might include safety, security or performance related. There are obviously many other types of analysis but they can generally be classified into one of these groups. The Project and People context have relationships with "provide product". 


\section{Project context}

The Project context is concerned with the management of projects.

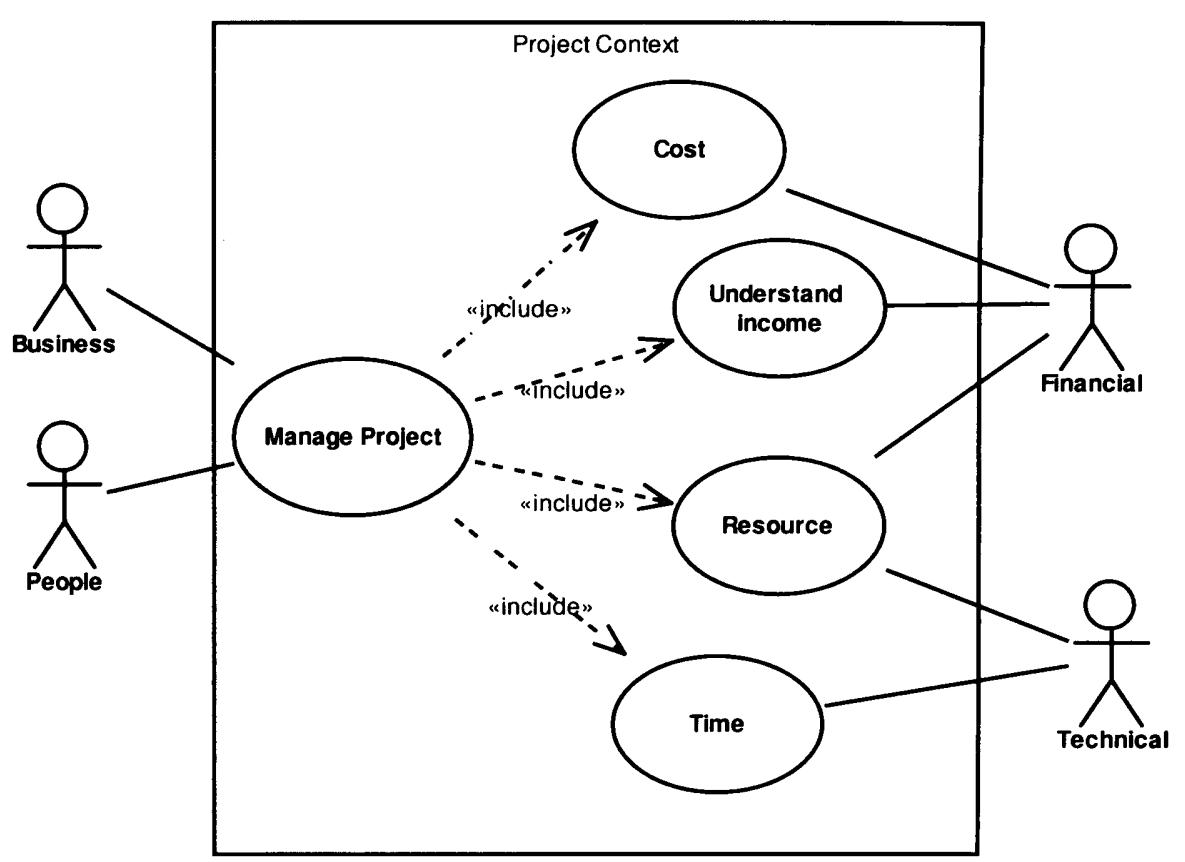

Figure 6-17 - Project Context

The Project context is focused on managing projects which includes managing cost, time, resource and understanding income related to the project. The Business context is related to the management of the project along with the People context as people carry out the project. The Financial context has relationships with the resource, income and cost aspects of the Project context and the Technical context relates to the time and resource aspects. 


\section{People context}

This final context looks at the individuals involved in the organisation.

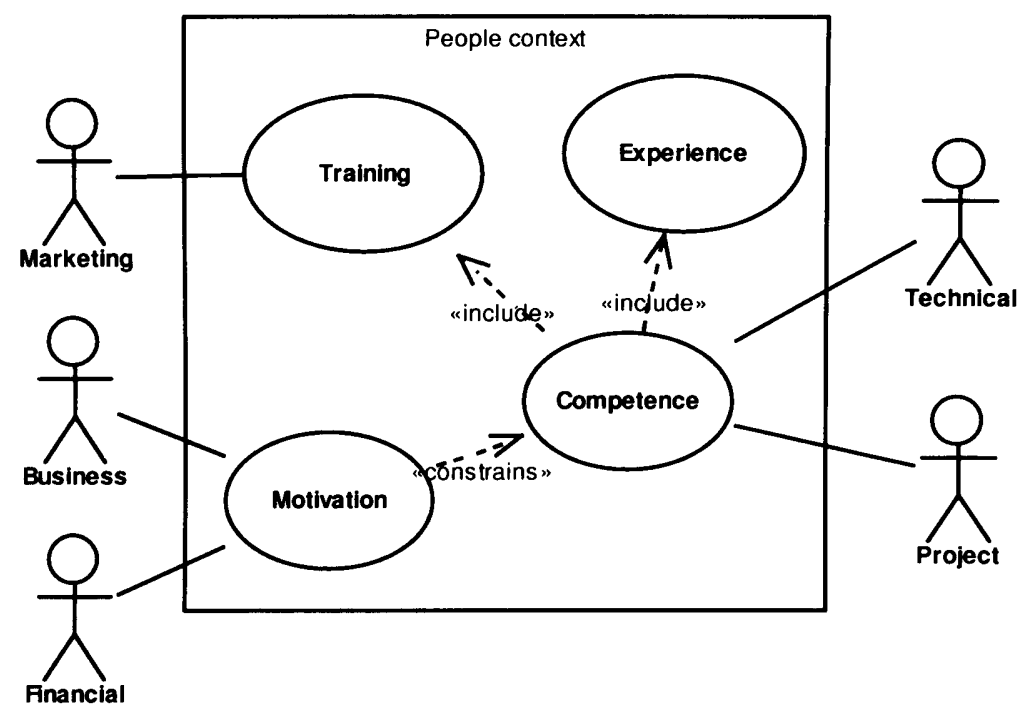

Figure 6-18 - People Context

The People context focuses on competence. Competence can be seen as the amalgamation of training or underpinning knowledge with experience or the skills developed through having applied that knowledge. A person's competence can also be affected by their motivation or attitude to work. The actors shown on this diagram cover all of the other contexts giving the People context the most complex set of interactions.

\subsubsection{Context Conclusions}

These six contexts provide a generic start point for understanding an enterprise however, they are not designed to represent any specific enterprise. As such they must be tailored to represent the enterprise in which risk management is being carried out. Approaches to the application of these contexts have been discussed in putting risk into context Brownsword and Setchi (2007). 


\subsubsection{Risk Evaluation}

This section provides further information on considering scenario analysis and the factors which cause and control risk.

The terms Hazard and Effect, from the previous chapter, are more relevant to the Risk Evaluation process and will be used within the example scenarios. It must be remembered that any Risk Evaluation will be relative to the viewpoint of the Risk Analyst and this is where the Tailored Context can be very useful for focusing the mind of the analyst to ensure that the right analysis is carried out for the right reason.

Scenarios provide a method of considering the behaviours that may lead to or result from a risk and we will discuss both a causal and effect scenario.

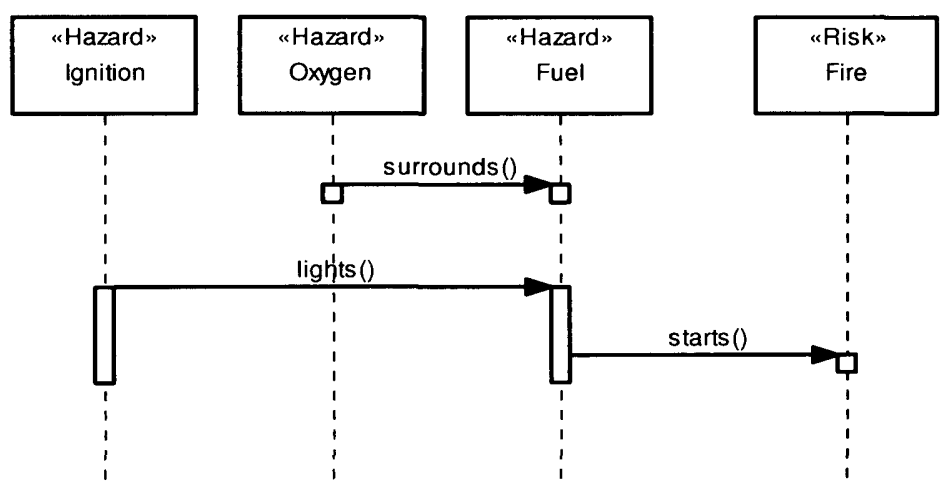

\section{Figure 6-19 - Risk Evaluation example Causal scenario}

Lets firstly consider a causal scenario in which Oxygen and Fuel come together with an Ignition causing a Fire. The scenario can be used in two ways initially. It provides an approach to understand how to cause fire this could be very useful in the event that we want to keep warm. It also provides a means to understand how to stop the fire occurring - remove Oxygen or Ignition or Fuel very useful for firemen to know when fighting a blazing house. This scenario provides a qualitative understanding of one cause of the risk Fire - there may be numerous other scenarios leading to Fire which also need to be understood. The initial understanding via qualitative means can be used to lead on to quantitative definition which can be used to gather snapshot and trend data related to the risk. 


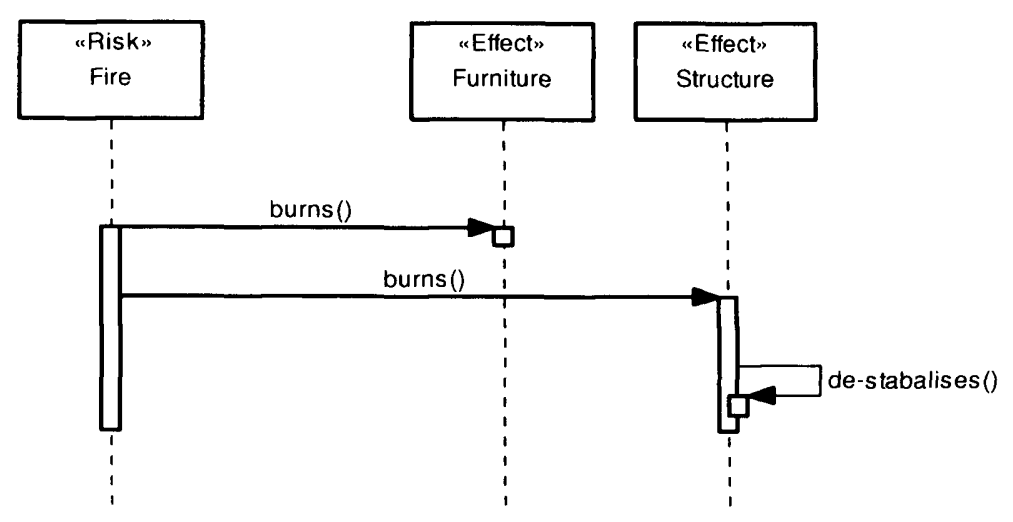

Figure 6-20 - Risk Evaluation example Effect scenario

Once the Fire is burning an Effect Scenario will show what happens next. In this case we will consider a Fire in a house fire, the Fire burns the Furniture and Structure of the house.

Again this provides a qualitative understanding of the effect of the Fire in this scenario, it is obvious in this case that we could move on to understand quantitatively the loss based on the occurrence of the risk. It is possible to use SysML parametrics to carry out this analysis in a bespoke manner as discussed in 'Formalising risk assessment through the use of SysML parametrics' by Brownsword and Perry (2009) however, SysML is not the only approach to carrying out the qualitative analysis but it does have the advantage of providing a Formalisation consistent with use of UML. 


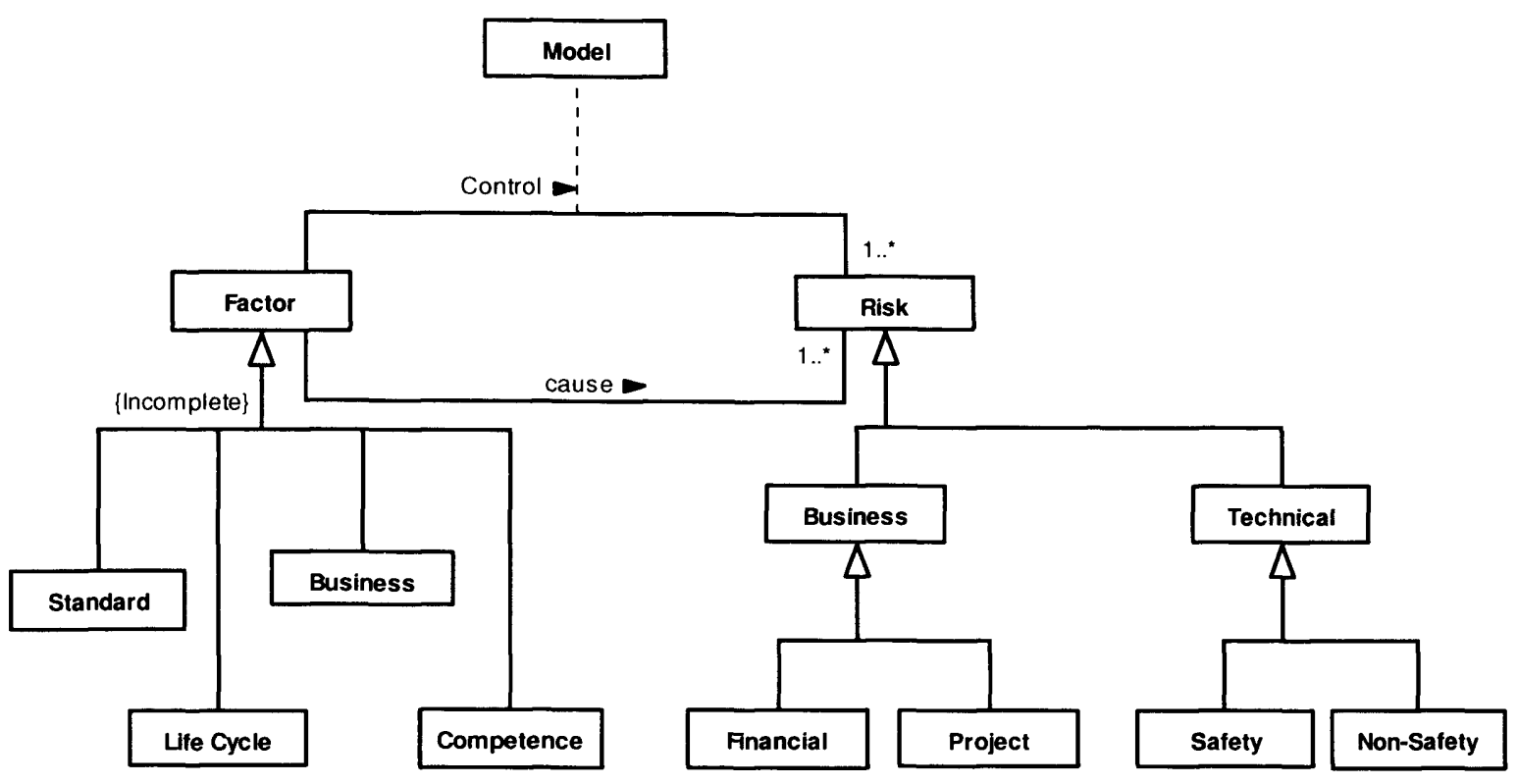

Figure 6-21 - Risk factor overview

There are a number of factors which may both cause and control risk, some of these factors are Standards, Life cycle, Business and Competence each of these affects different and sometimes identical categories of risk discussed further in picturing risk Brownsword and Setchi (2005). Each of these factors can be considered at any point within a cause or effect scenario. These factors are similar to the categorisations within the Taxonomy-Based Risk Identification report by Carr et al (1993) where they have identified factors within each of the basic engineering processes and are used in a similar way, to support causal analysis.

\subsection{Conclusion}

The purpose of this chapter was to define the processes required to carry out risk management using a multi-view approach. This has been achieved through the application of the 'Seven Views Approach' which has been used to formalise and communicate the processes for risk management whilst providing a consistent method to identifying concerns, defining risks and evaluating their cause and effect. 


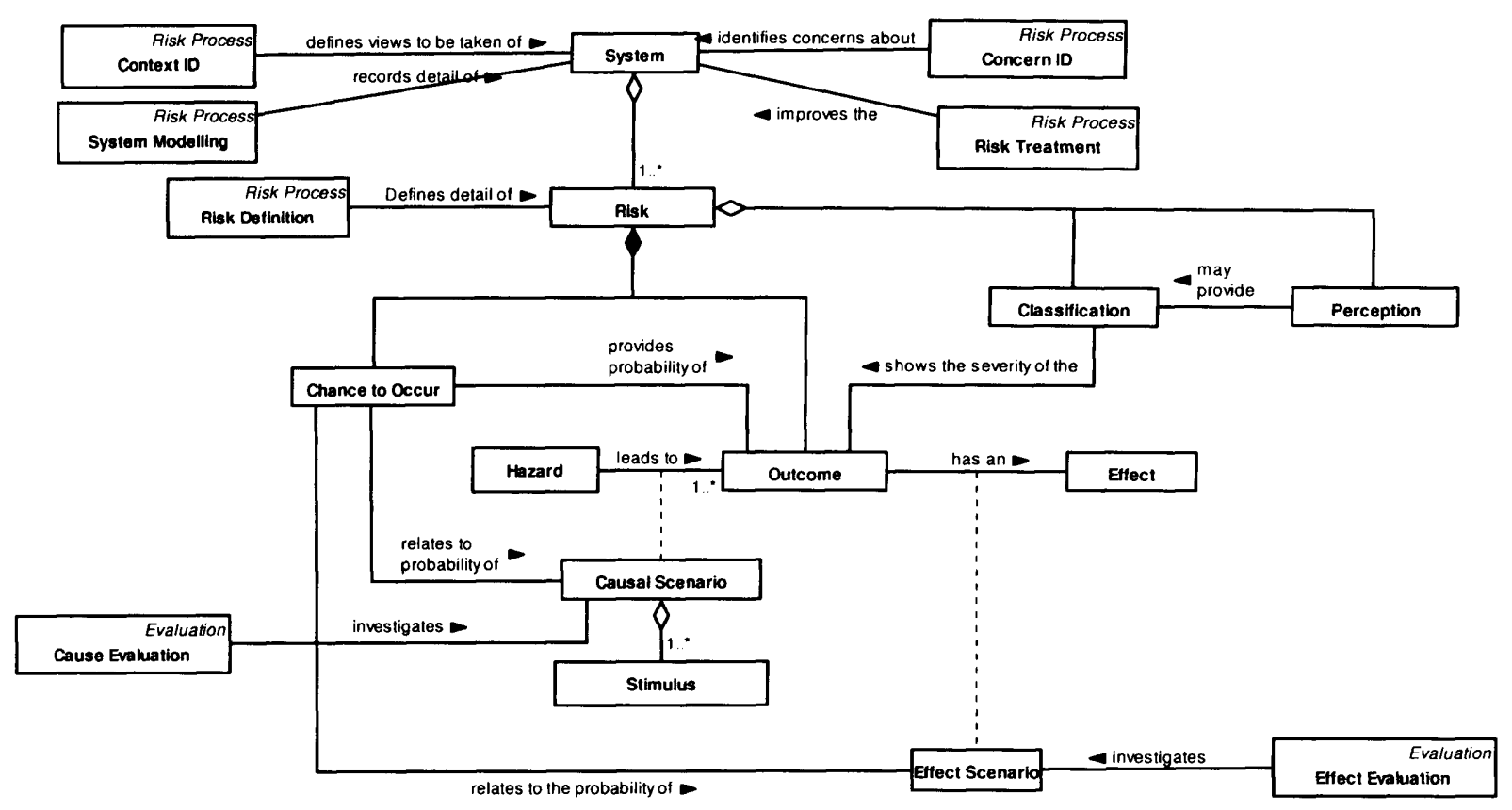

Figure 6-22 - Risk Ontology with processes

By providing these processes in a common language which is widely accepted for process modelling and mapping them to the terminology defined in chapter 4, Figure 6-22, a clearer understanding of a risk and the management of risk can be understood. This may be applied directly in industry, as is the case with the FDF/Seafish or for teaching - allowing students to better understand and question the terminology that they are presented with.

Using this common language to describe the output of the processes as well as the processes themselves is a major improvement enabling consistency across process and implementation.

The following chapter will detail examples of the application of the processes, contexts and taxonomy defined here. 


\section{Case Studies}

\subsection{Introduction}

A number of Case studies have been carried out during this work to support the definition, development and evaluation of the Ontology and Processes. The case studies have been carried out using a variety of applications to ensure a general nature in the processes and definitions already presented.

The objective of this chapter is to demonstrate the applicability of the processes and ontology. This objective will be achieved through the presentation of two paths through one of these case studies showing the processes applied in a pragmatic but formal manner. These paths are based on the application of the Ontology and processes to a charitable organisation. The first path looks at a health and safety concern and the second at a governance concern. The application of the processes to this organisation shows the benefit of using a formalised approach to the management of Risk and the ability of the processes to apply to different types of Risk.

\subsubsection{Background}

Charitable organisations must not only ensure that they are financially viable (that they have a business case) but also that they meet the needs of the beneficiaries of the charity while conforming to the rules set out by the charity commission.

Kings Norton Parish Church, winners of the 2005 BBC 2 Restoration programme, is such an organisation. Not only is it the custodian of two lovely restored buildings it is also a team parish, meaning that there are a number of staff members (clergy) to look after four churches, their congregations and to spread the word through the wider public.

The Kings Norton Parochial Church Council (KNPCC) has identified the need to improve the way in which risk management is carried out. 


\subsubsection{Case study tactics}

This study will be carried out using an ethnographic/participant observer approach discussed by Yin (1998), the researcher is already an established member of this community. Participants in the study will not be aware that research is being carried out.

The study will record the use of the ontology and processes defined for risk management. The application of the processes will be led by the researcher along with a small number of other members of the community, these members understand the need to carry out risk management and are happy to trail the processes. The data created through the use of the processes will be collected in two ways. General knowledge regarding the activities and relationships within the organisation will be stored in a relational database whilst information regarding the risks will be stored in the organisations enterprise architectural/risk management model.

The main aim of this study is to understand the practicality of using the ontology and processes, understanding firstly whether the processes can be applied in a pragmatic way and secondly whether they are flexible enough to deal with changes in the current situation in a timely fashion.

A benefit to this study will be the lack of existing risk management processes within the organisation. This lack will reduce the cultural change issues associated with the implementation of different approaches.

It is recognised that bias maybe introduced into this study due to the researchers ability to guide events. This will be mitigated through the use of a team approach to the approach followed ensuring that the processes and ontology are not forced onto the work which is to be carried out. 


\subsection{Approach}

The processes have been applied using the more pragmatic ordering recognising the fact that work has been carried out before and that there is some understanding of the concerns that exist. For this reason the case study will be discussed in three main sections.

1. The initial processes carried out identify the first set of concerns, contexts and risks. The risks which will then be taken forward and discussed further are an health and safety issue and a governance issue.

2. The health and safety risk shows the consideration of people's needs in relation to the physical structure of the building and although other concerns are raised which could affect the solution the processes are adaptable enough to include concerns from other contexts.

3. The work to solve the governance risk shows how information can be recorded and retained to enable future concerns and risks to be evaluated in a more efficient way, it also supports the definition of a parish wide mission and the definition of job specifications for clergy.

Each section will be preceded by a sequence showing the order in which the processes from Chapter 6 have been implemented.

The first of these sequences can be seen in Figure 7-1 below.

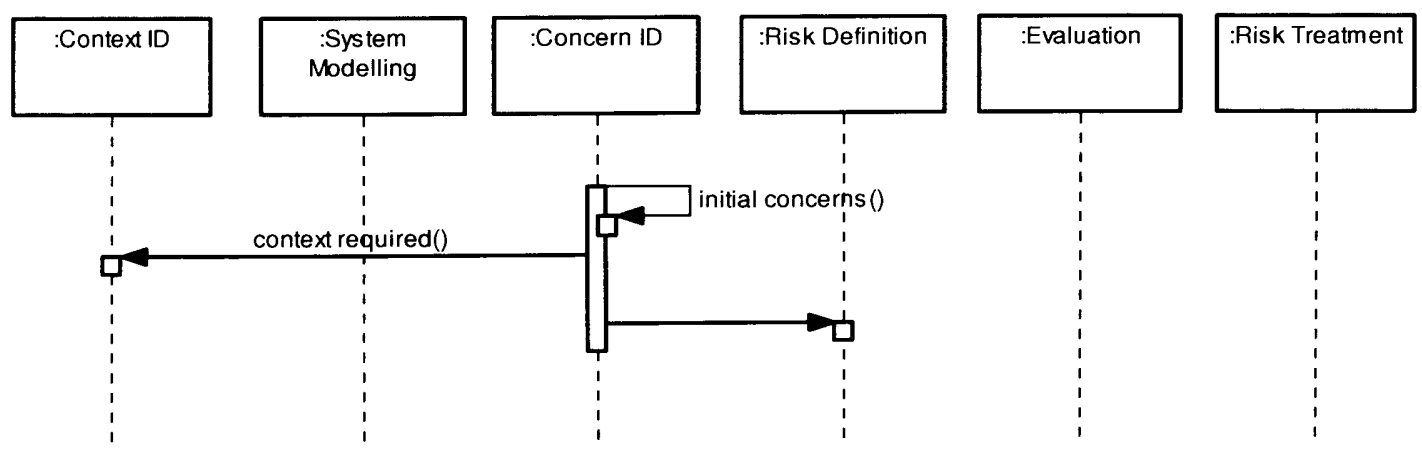

Figure 7-1 - Initial Process Instance View

This work starts by executing the Concern ID process, it is expected that this 
in truth is where most applications will start, one of the reasons for this is that it provides direction and scope to begin where contexts and systems models do not exist, this will then be expanded in future iterations to encompass other areas of concern. Following the initial identification of concerns high level contexts are suggested before ensuring that any immediate risks have been defined.

\subsubsection{Initial Concern Identification}

The initial concerns were elicited during a meeting of the KNPCC (as the governing body of the organisation).

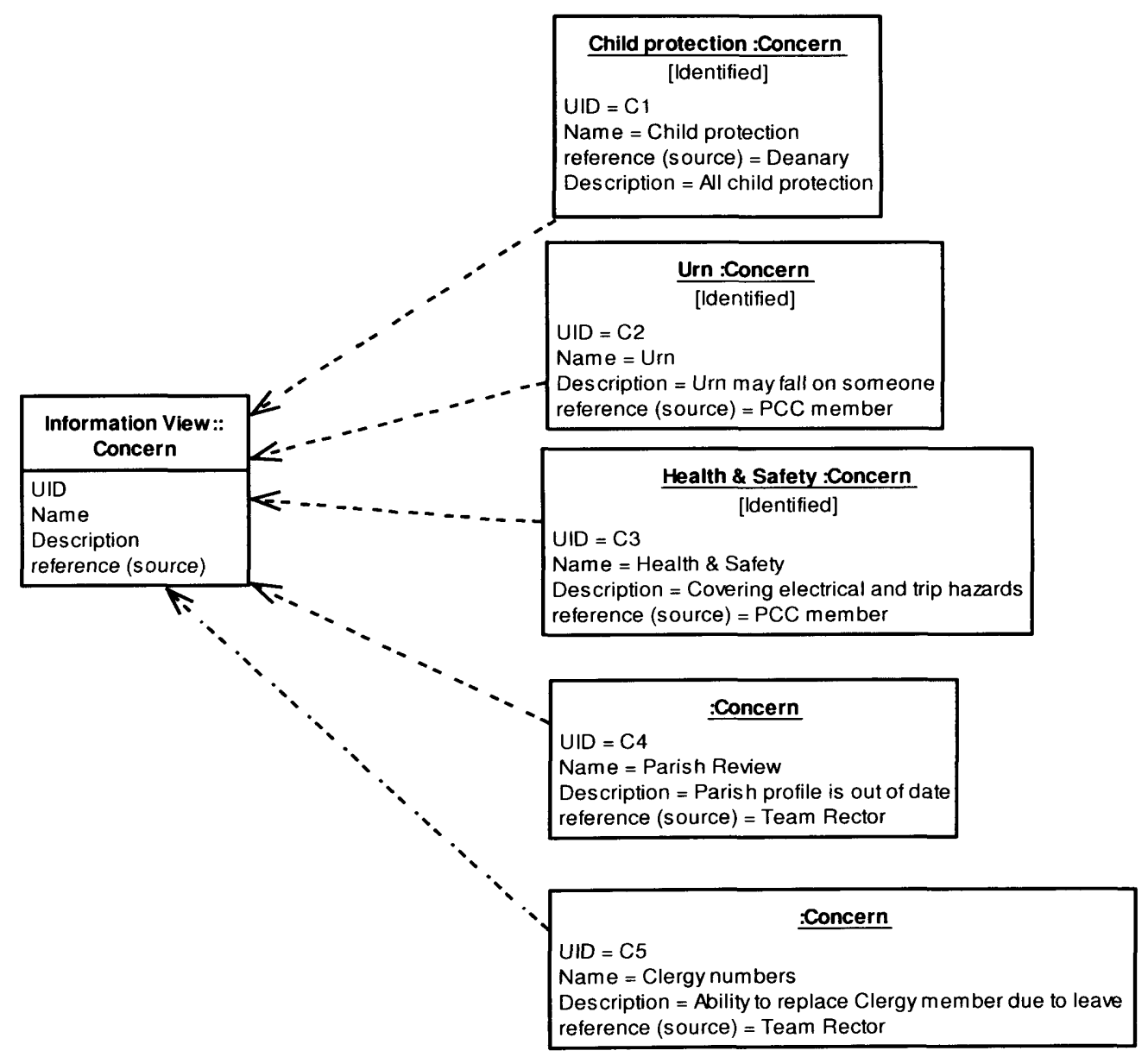

Figure 7-2 - Initial concerns

It can be seen that the initial concerns, Figure 7-2, vary in scale including governance, staff numbers, health and safety (a matter for any organisation with buildings and people) to an Urn. An explanation was required to understand what the Urn concern was, it suggested some large pot waiting to 
fall on some unsuspecting passer by, in actual fact it is a hot water urn which is located near a children's play area and could be occasionally left unattended whilst the children play. This is obviously a health and safety issue although focus is required, analysis and categorisation of concerns will take place at another point.

It is imperative to ensure that the terminology used throughout the application of the processes is consistent with that defined in the ontology. Here the term Concern is being used and the direct reference from the Information View for the processes has been highlighted, the reference helps to ensure that the correct terminology is used at all times.

\subsubsection{Initial Context Development}

With a number of concerns captured an overview of the organisation was required to give some scope to these concerns. A number of contexts in which the organisation works gives some focus to this and will enable a considered approach to the definition of structure and behaviour during future process execution.

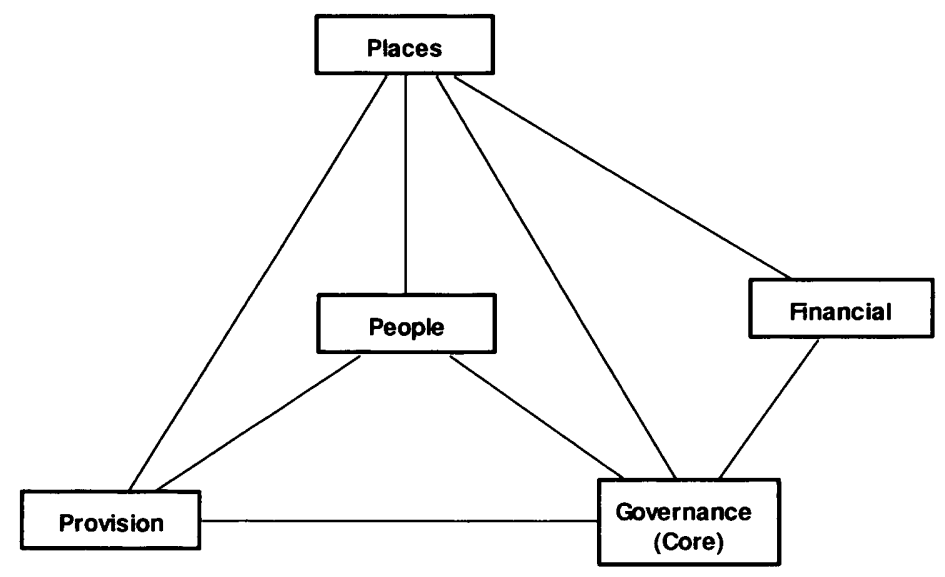

Figure 7-3 - Context identification

In this case only the high level contexts have been defined initially, Figure 7-3. These provide a start point for further consideration once a better understanding of the organisation has been achieved. These contexts may also be used to focus future work so that it considers specific areas in which the organisation works rather than floating around ideas which may never bear fruit. 


\subsubsection{Initial Risk Definition}

In the first instance the risks identified are the current priorities based on the situation that the organisation is in and those which are likely to show results in a reasonable time frame.

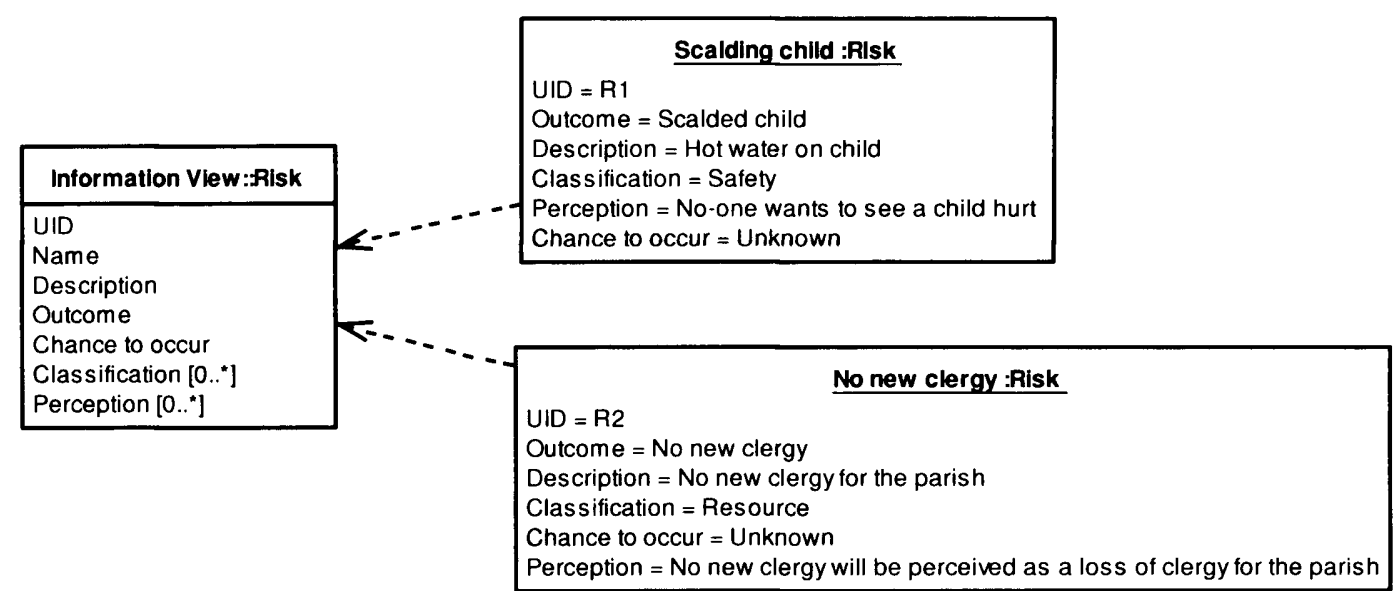

Figure 7-4 - Risk Definition

Again terminology is key, at this point Risks are being defined and the terminology used to define them must be from the Information View and therefore the Ontology. It is interesting that in both these risks, Figure 7-4, the Chance to occur is unknown this will be the case with many non technical risks as it is almost impossible to calculate the probability and where it is possible it tends not to be accurate. The only way to get close would be to base the chance to occur on a fraction of the number of children recorded to have been scalded in a set time frame, or to ask when it last happened in this building or those like it. The problem with all of these approaches is twofold; firstly there are so many variables that the chance to occur that is recorded will not reflect the true chance to occur of that outcome and secondly the time spent calculating the chance to occur is wasted as the perception and classification of the outcome mean that something will be done whatever the chance to occur actually is.

Having defined the risk it is also worth recording where it came from, this may be a relationship to part of the system model, context, another risk or concern. 

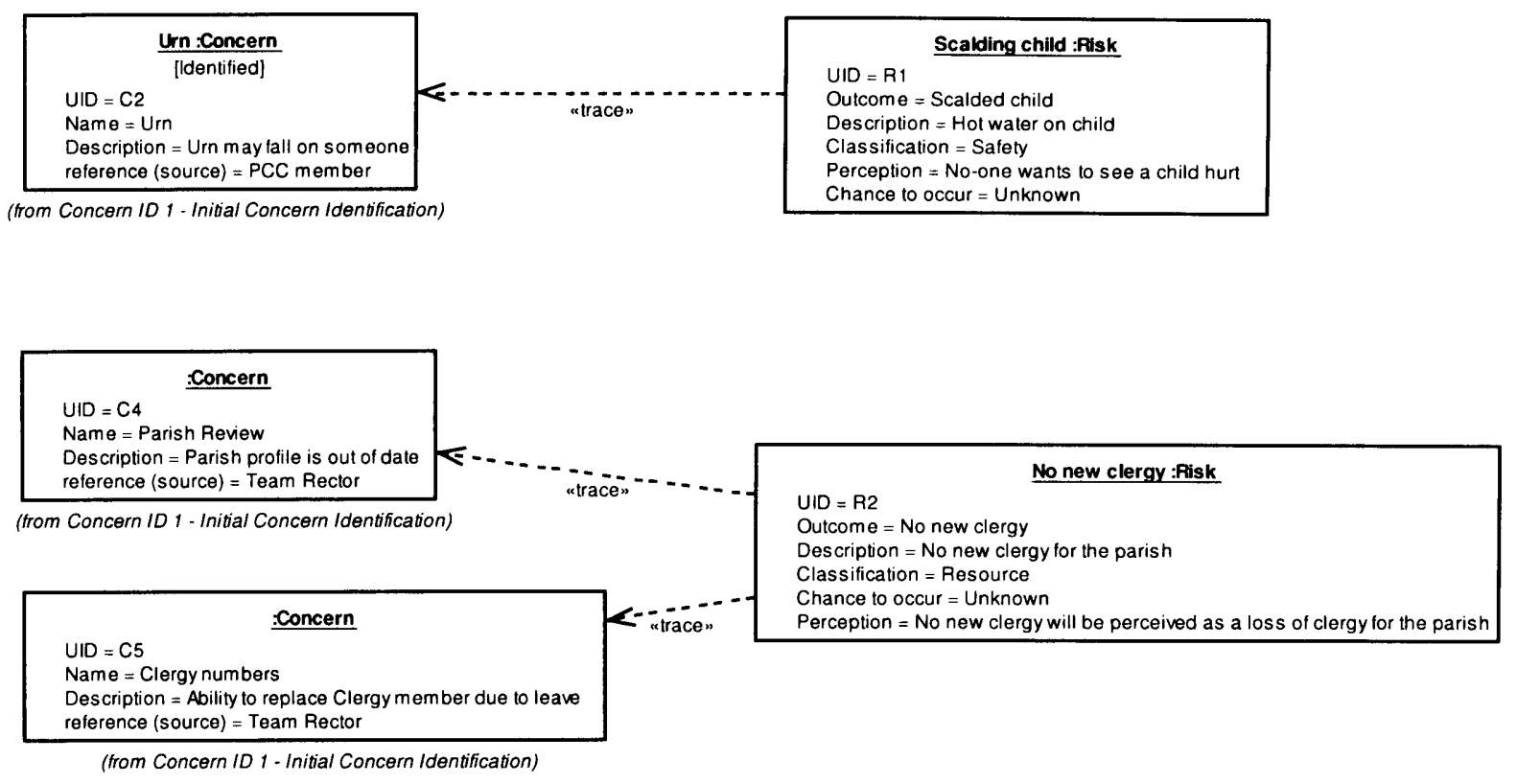

Figure 7-5 - Risk - Concern Trace

In this case, Figure 7-5, both risks are based directly on the concerns that were raised. Although this is the case currently it is possible that other areas, structures or needs will be identified later that either re-enforce the definition of the risk or affect one of its attributes, possibly the perception or categorisation. This supporting information can be added when it is identified either during the execution of the systems modelling and context id processes or when next the risk definition process is carried out.

At the conclusion of the initial process execution a number of concerns, contexts and risks have been identified, each may raise many questions. The following will begin to ask some of these questions by both re-applying the processes used here and expanding the investigation through the evaluation and treatment of these two Risks.

\subsection{Health and Safety Risk}

The first path to be presented will consider the case of a child being injured through scalding. The development of a system model will enhance and support the evaluation of the cause and effect of the Risk before solutions, if required, are considered.

The added complication of extra concerns being raised which affect possible 
solutions to this Risk will also be discussed and an approach to incorporating these described.

Changes happen even in the most stable of situations, in this case the expected processes have been presented enabling a comparison later when extra inputs threaten to unbalance the approach.

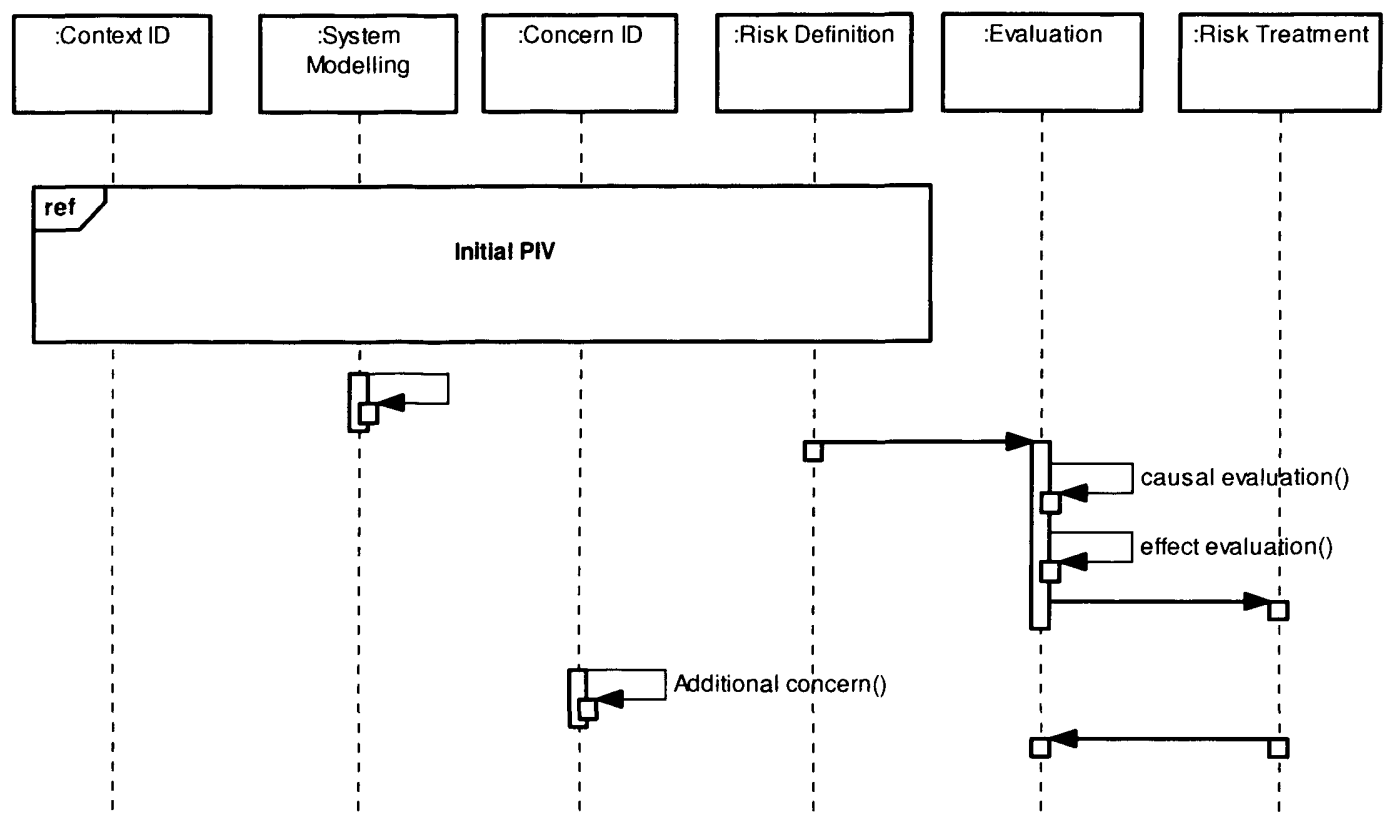

Figure 7-6 - Scalding child Process Instance View

Following the initial processes, already covered, more detail was added to the areas directly related to the 'Scalding Child' risk, Figure 7-6, before re-visiting the definition of the risk to ensure all areas had been covered in the depth available. Both cause and effect evaluations were carried out based on the system model before considering changes to the system model that may provide solutions to the Risk.

\subsubsection{Understanding the System}

Understanding the current situation and recording the terminology behind it are the objectives of this execution of the system modelling process. In this case the process will firstly look at the terminology before organising the terms to create a representation of the current situation. The focus of the process will be within the places context that is focusing on the buildings areas within. 
It is important to understand the terminology and concepts currently in place as these will provide the consistency behind any populated views or representations of the current situation. Ideally this information would already exist within an Enterprise Architecture (EA) as described by (Zachman 1997) or (Holt 2009) however, in this case there is no such EA, much of the information is tacit knowledge, as such the process will be used to record the information rather than to identify from existing sources. The process will begin by recording some of the terminology used within the organisation.

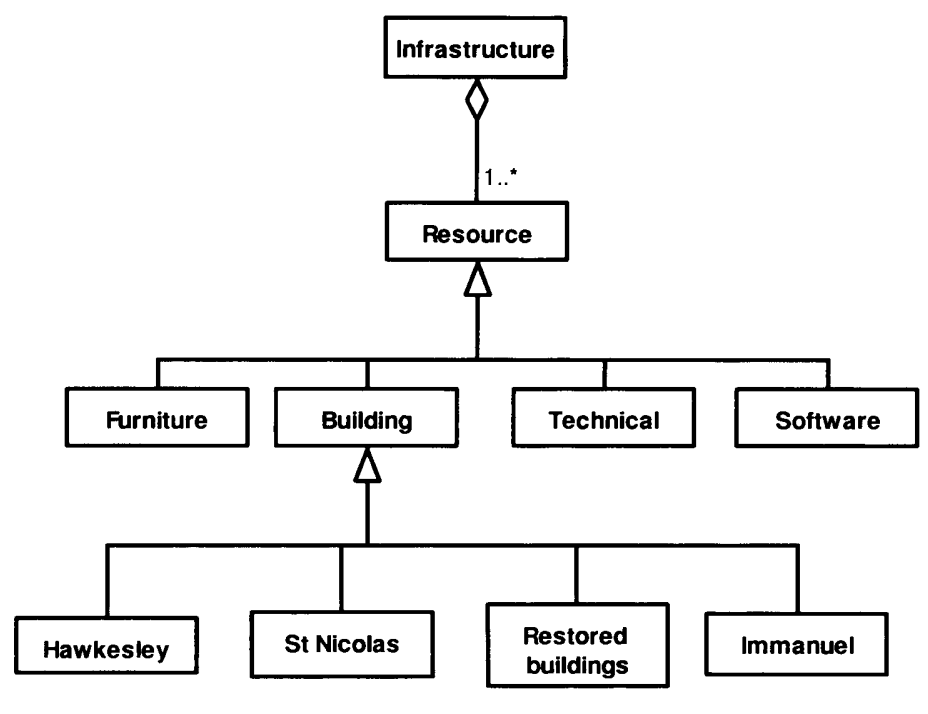

Figure 7-7 - Infrastructure

It is important to understand the concepts within the infrastructure to define what the organisation has before trying to arrange it and place it in specific locations. It is also important to know that the organisation does not own all of the resources that it uses, some of them are hired and as such only available at specific times and places.

Buildings are of obvious importance, the diagram in Figure 7-7 aids in the understanding of the buildings which are considered to be of relevance, this means that there may be other buildings not shown in this diagram but could be equally as important in another view. 


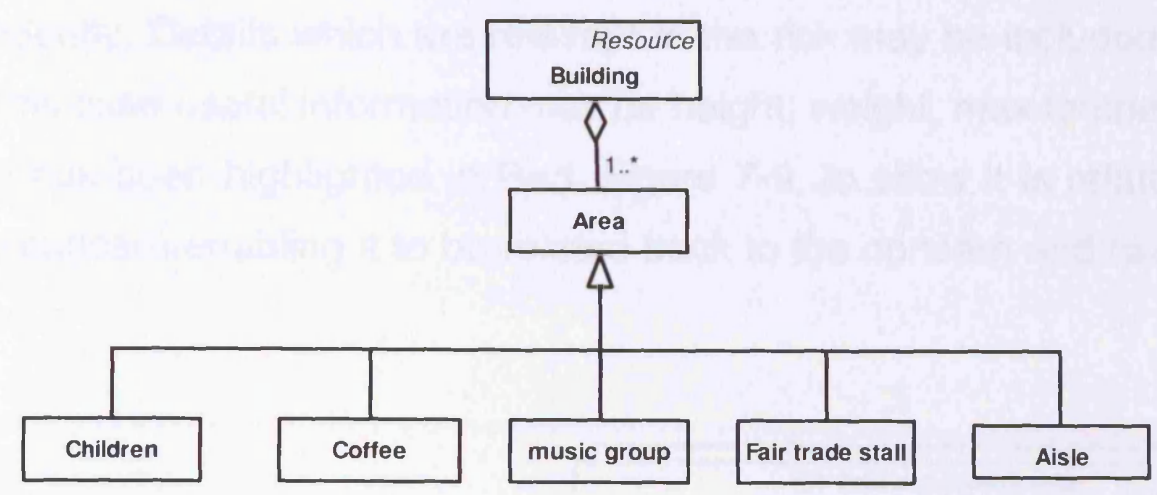

Figure 7-8 - Areas within buildings

Within the buildings themselves there can be designated areas, Figure 7-8. These areas have their own needs, again a complete picture will be built up over time with areas added as required. The coffee and children's areas are the two that are of specific note here but it is useful to record as much information as possible to help with future work and to understand the language and groupings within the organisation.

Having defined terminology it is possible to populate views with information representing real situations. A populated view uses the terminology defined to build up a picture of the location in which the concern, the Urn, and the Outcome, a scalded child, reside. In this case this means focusing on the furniture supporting the Urn, the area that it is within and other surrounding areas.

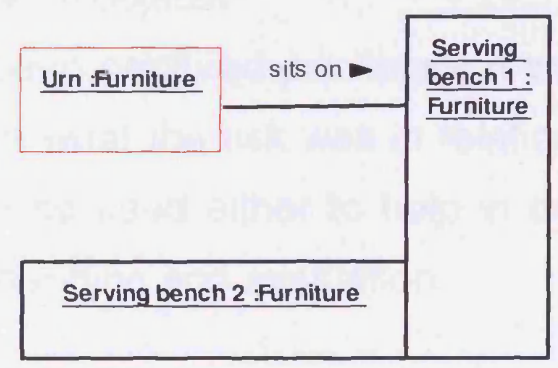

\section{Figure 7-9 - Population - St Nicholas Urn Concern}

The Urn itself is a large metallic container which holds, heats and boils water. It is located on a serving bench which has another bench adjoining it. It is useful to note that none of these items are secured and each can be moved 
independently. Details which are relevant to the risk may be included for later use, in this case useful information may be height, weight, max temperature. The Urn has been highlighted in Red, Figure 7-9, to show it is related to the risk and concern enabling it to be related back to the concern and risk directly if desired.

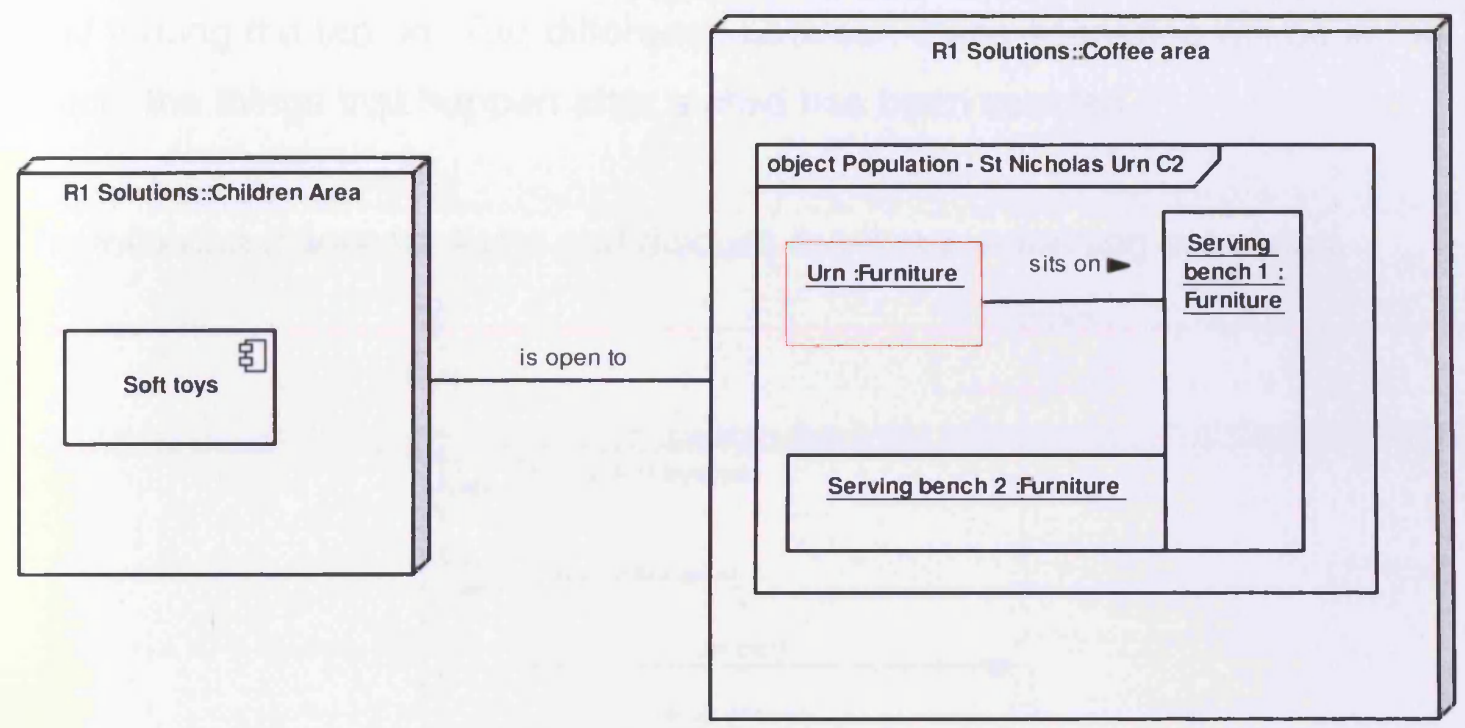

Figure 7-10 - Population - Urn in area

Figure 7-10 shows the two of the areas within the St. Nicolas building, more importantly it shows their proximity to each other, it is also a good example of how concerns may manifest themselves on these diagrams when the concerns related to physical objects.

If this information had been captured previously it would have been used as part of the decision as to what the risk was in relation to the Urn. This shows that the information can be used either to help in the elicitation of concerns and risks or their understanding and evaluation.

\subsubsection{Scalded child situation evaluation}

The following evaluations show the behaviour that may cause the outcome to occur and the effect following an occurrence.

A number of scenarios were considered for causal evaluation these included: 
- A child pulling the tap on urn

- A child pulling tap on urn after moving table (barrier)

- A person falls into the bench and knocks the urn off.

- A child pulls urn over

It was agreed that a child pulling over the urn follows the same sequence as a child turning the tap on. The difference between these scenarios will be in the effect - the things that happen after a child has been scalded.

The following diagrams show and discuss the three remaining scenarios.

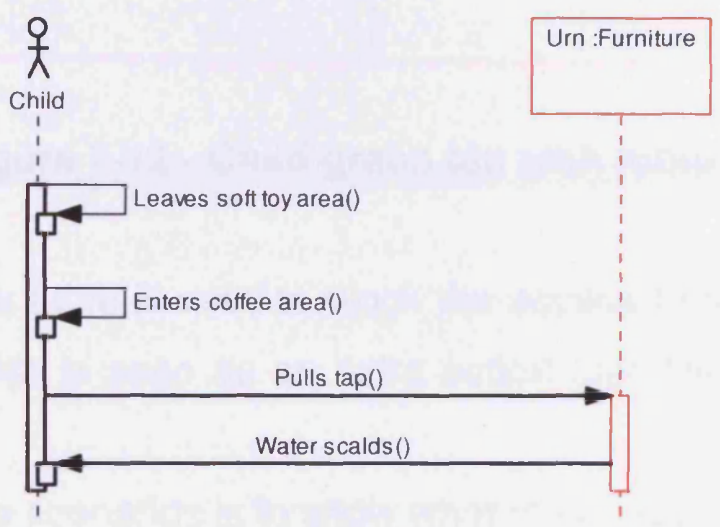

Figure 7-11 - Child grabs tap on urn

In the simplest case, Figure 7-11, a child may leave the children's area move directly into the coffee area and pull the tap on the Urn. This case assumes that the child can reach the tap. The most remarkable thing in this scenario is that there is nothing to block the path between the two areas. 


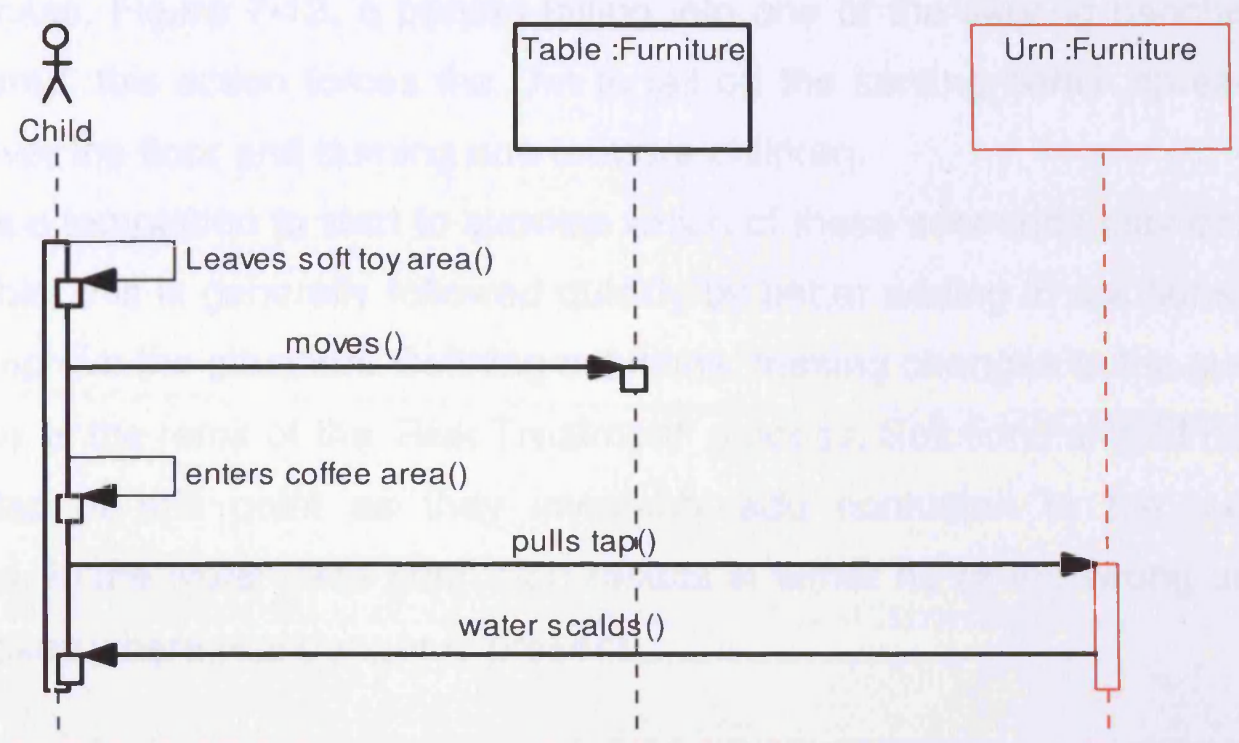

Figure 7-12 - Child grabs tap with table

On some occasions a table is used to block the access to the serving section of the coffee area, this is seen as an extra action that the child will have to perform, Figure 7-12.

The intention of these scenarios is to show what may happen in the lead up to the outcome occurring. These scenarios may in future uses of the processes be used to inform more detailed and even calculation based information, but it must be remembered that they only show one possible route rather than all possible routes to an outcome, hence the presentation of three scenarios giving a wider understanding of the different stimuli which may lead to the outcome.

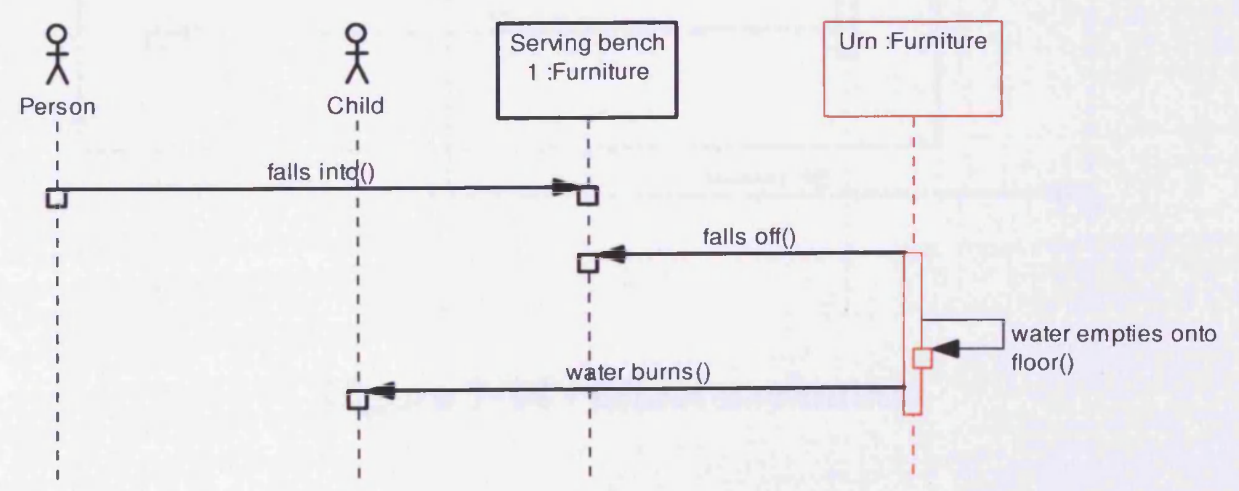

Figure 7-13 - Person falls into bench 
In this case, Figure 7-13, a person falling into one of the serving benches is considered, this action forces the Urn to fall off the serving bench spreading water over the floor and burning one or more children.

There is a temptation to start to surmise which of these scenarios may best or preferable, this is generally followed quickly by better adding in solutions that could improve the situation. Defining solutions, making changes to the current situation, is the remit of the 'Risk Treatment' process. Solutions should not be presented at this point as they invariably add confusion to the current situation. In the worst case confusion results in either no or the wrong action being taken where real danger is present.

\subsubsection{Effect evaluation}

The effect evaluation looks at the events that follow the occurrence of an outcome, again this should reflect the current practices or procedures. It is still likely that more than one scenario will be expected for the effect as in most cases there will be more than one way to address the outcome.

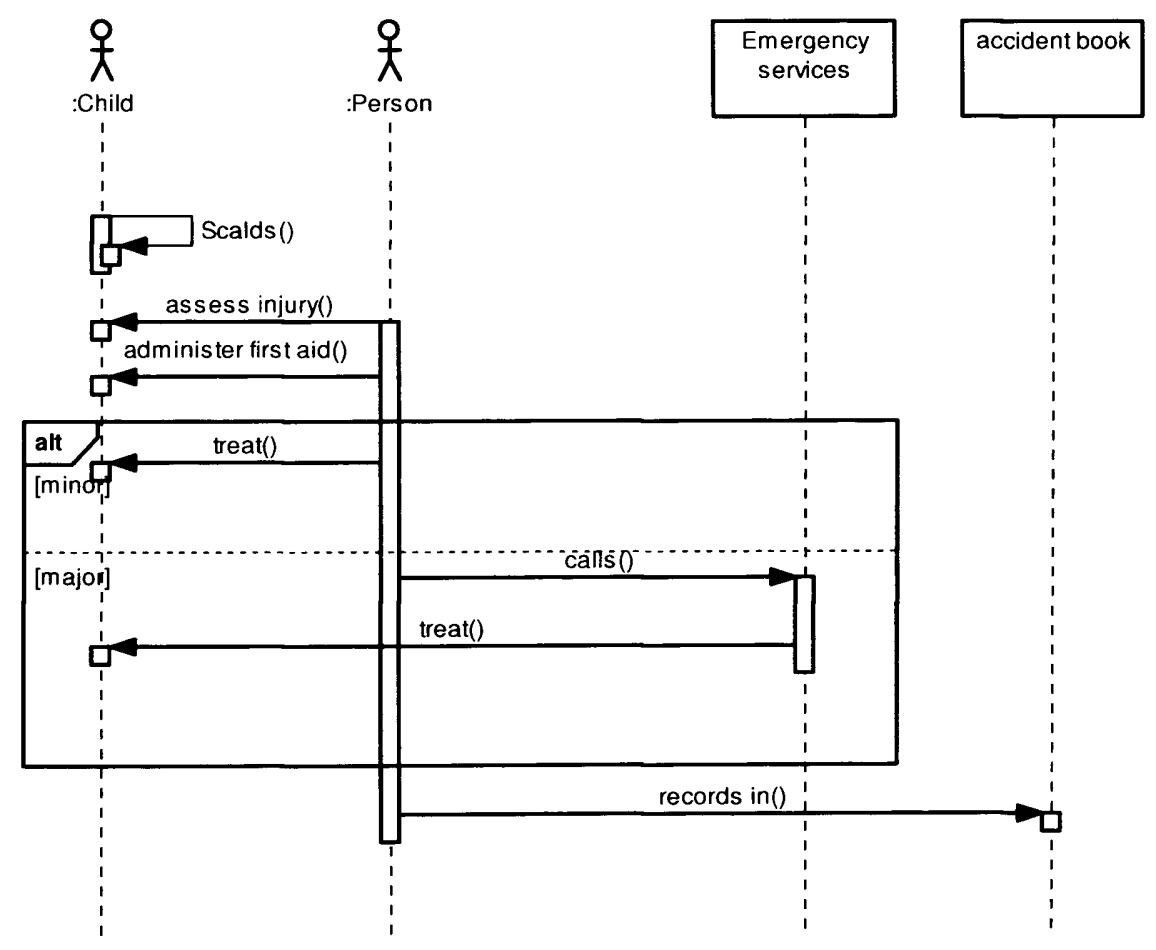

Figure 7-14 - Effect evaluation

There are two ways in which burns can be dealt with in this organisation; the difference between them is shown in the 'alt', alternative section of Figure 
7-14. For minor injury the first aider will treat the child where as for a major injury the emergency services will be called. Incorporating two options in a scenario in this way can be very useful for small variations, however when larger or more complicated alternatives are possible they can become unwieldy - adding complication rather than clarifying a situation.

It was expected that before any evaluation was carried out more definition may be added to the risk however in this case no information that will improve the definition of the risk has been identified.

\subsubsection{Treating the risk of scalding a child}

Having identified both causes and effects of the outcome the risk treatment processes uses the preceding information as a basis to decide whether mitigation, solution or treatment should be considered. Treatment could mean changes to either cause or effect, the ideal is to remove the possibility of the Outcome or make the Chance to occur zero. When the chance to occur can not be reduced to zero treatments ensure that sufficient procedures are in place to be exercised should the outcome occur. As this outcome could cause serious injuries resulting in the attendance of the emergency services it is agreed that treatments for the risk should be considered. Seven possible treatments have been identified;

1. Current (with table to reduce access)

2. Remove urn (move to another location)

3. Soft area in North pews

4. Soft area in South pews

5. Secure serving furniture and add door

6. Replace urn with fixed plumbed boiler on wall

7. Do not leave urn unattended.

By way of this example two of these options will now be discussed further, these will be options 4 and 6 . 


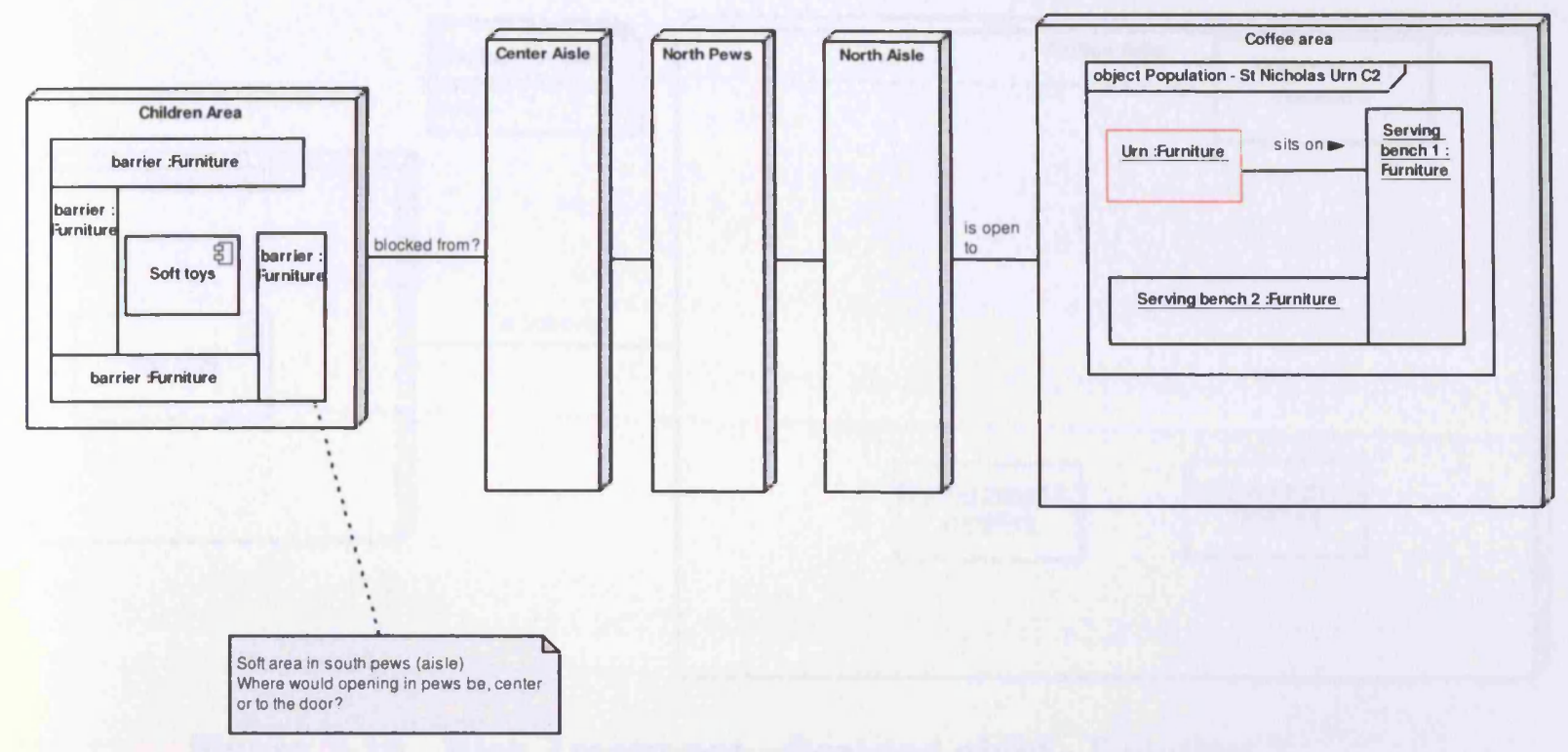

Figure 7-15 - Risk Treatment - Scalded child - Solution 4

This solution, Figure 7-15, shows the use of other areas within the church to provide a separation between the children's area and the coffee area. These solutions have been discussed with affected parties and questions raised. Questions such as, will the main entrance door and step attract children and present concerns, what sort of barrier will be used, is it a problem that this will increase the distance to the toilet and the quiet area where carers take crying children. The fact that these queries include items or areas not shown on this diagram is not a problem; quite the reverse the identification of the areas will support the improvement of the system model in another iteration of the process. 


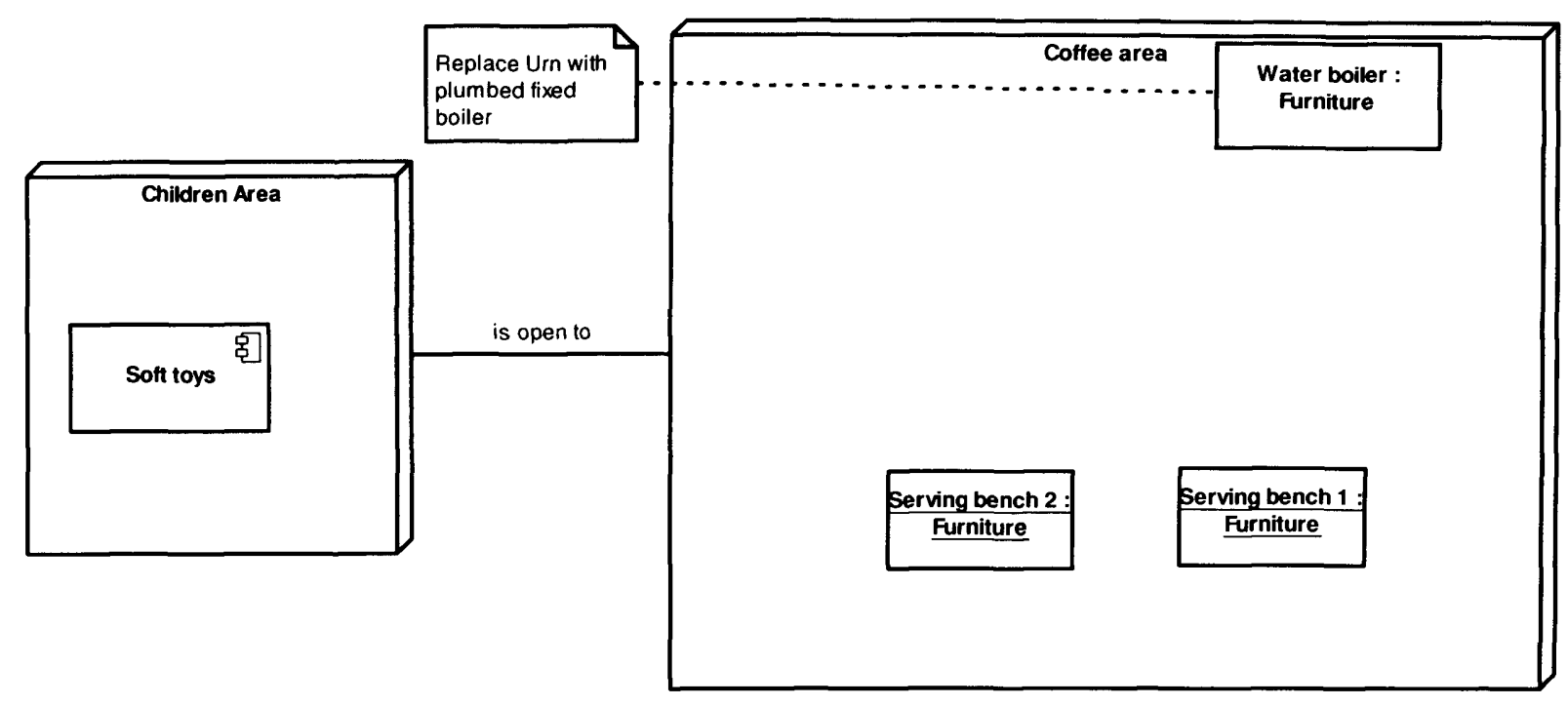

Figure 7-16 - Risk Treatment - Scalded child - Solution 7

Suggesting that the urn should be replaced with a fixed boiler, Figure 7-16 met with resounding agreement, rather than issues or questions being raised statements were along the lines of; won't this be more environmentally friendly, this will save others having to move the urn to empty it, this will save money, the only negative point was that holes would have to be created in the wall to enable fixing and plumbing.

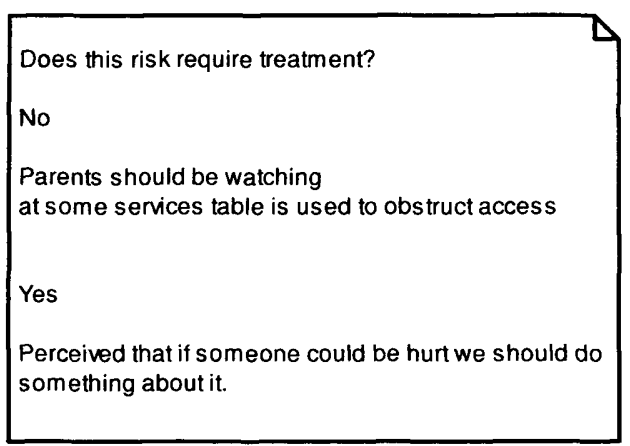

Figure 7-17 - Treatment - Scalded child

The decision now has to be made whether to treat this risk or not. There are arguments for both for and against including:

For - perception that if someone could be hurt something should be done about it.

Against - Carers should be watching, in some situations a table is used to 
obstruct access.

Both of these options could provide a reasonable solution in the way of a treatment for the risk, however to select a solution at this point would be premature. These solutions have been defined to solve the problem from the context of the place or building, their impact on the other contexts has not yet been considered.

\subsubsection{Additional Concerns}

This case study was all going well, it was selected as a 'simple' concern to look into, it was according to plan, everyone was happy... and then, as happens with most projects, plans and operations something else cropped up. Another concern was raised which was obviously linked to this risk. The concern was about the amount of space available in the children's area, more importantly one of the provisions is a group which has outgrown the space. It was recognised that this concern may increase the chance to occur posed by the 'Scalding child risk' as tiny tots, the group, was regularly having 18 children in the space whilst the urn was on.

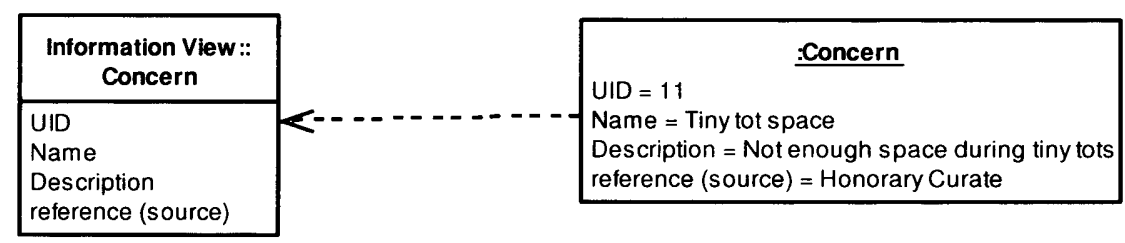

\section{Figure 7-18 - Concern Identification Revisited}

This concern, Figure 7-18, had the potential to throw the work out of control as it may bring in other concerns and the treatment work may have to be reiterated or completely re-done. Using the modular processes and robust set of terminology defined in this work means that this is not the case. Using processes in this way anticipates changes and additions such as this and in this case it could even be considered to be timely providing needs for the provision context against which solutions will be evaluated. 


\subsubsection{Scalded child Solution evaluation}

The provision context is directly related to the additional concern and as such provides extra needs which to be considered. The main focus here is on available space and this is two fold, there must be more physical space for the children's area without reducing the overall seating capacity of the building. Therefore each solution will be evaluated using parametrics based on number of seats and amount of open space.

Most of these solutions do not have any relationship with finance as there will be no capital outgoings. There is one however which does; solution option 7 suggests the purchase of a fixed and plumbed boiler unit and the financial implications of this must be understood.

The cost of a boiler of this sort is in the region of $£ 400$ however the running cost should be lower balancing this out over time. These costs including fitting will need to be considered if this is thought to be a viable solution.

Something happening to a person in a place was the original reason for the identification and definition of the concern and risk. From this point of view this context can be considered to have been covered. It is worth however looking a little further at this as there are a number of points to understand.

1. this risk supports the definition of a relationship between the people and places contexts

2. other people interact with the urn, not just children

There is no direct relationship to governance as this is a place and people issue and the effect on the contexts are not significant enough to have repercussions through all contexts at this point.

\subsubsection{Health and Safety Risk Conclusion}

Solution selection is not within the remit of risk management only the provision or suggestion of solutions. The selection of a solution must be the responsibility of the whole organisation working with the broadest knowledge of what it is trying to achieve. As such risk management provides the 
background information material and ability to monitor treatments.

In this case the solution selected by the KNPCC due to the additional size concern presented by the tiny tots group was a joint solution that of solutions 4 and 7 . This provided the best all round solution as it gives more space to the children's area and therefore the tiny tots group whilst also moving them away from the hot water supply. The installation of a fixed boiler also helps to move the boiling water out of the reach of children whilst saving the organisation money overall and on a rolling basis. Currently solution 4 has been implemented.

\subsection{Parish review}

The second path through the case study looks at a governance and mission concern covering the need to understand the remit, structure and working of the whole organisation.

This path through the case study has been defined to provide a solution, however as the study progresses it becomes obvious that the solution to this concern and risk will identify a host of others. This is expected in the early stages of Risk Management. A boom in the number of concerns and Risks at this point can be considered healthy. The expectation then is for the rate of increase of concerns and risks to reduce in time.

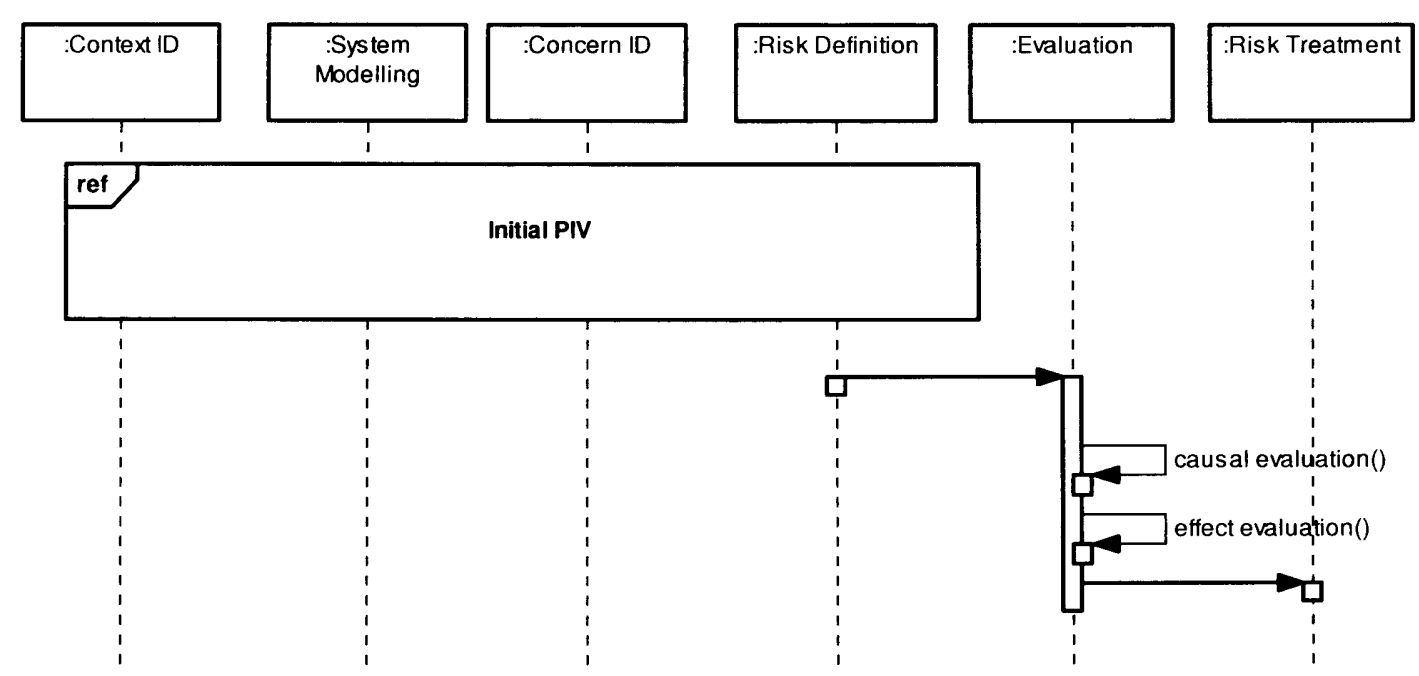

Figure 7-19 - Parish review methodology 
It is envisaged that the solution to this Risk will be a project, Figure 7-19, and as such the solution will not be evaluated against all contexts in this example, this will occur during the project itself by the nature of the work to be carried out.

\subsubsection{Situation evaluation}

In constructing the causal analysis the point at which a Clergy member leaves a parish provides a starting point, why they choose to leave is not under investigation. The investigation is to the cause of clergy members not being replaced in this team parish. In a team parish an assessment is carried out after a clergy member leaves and a clergy member leaving does not mean that there is a vacant position.
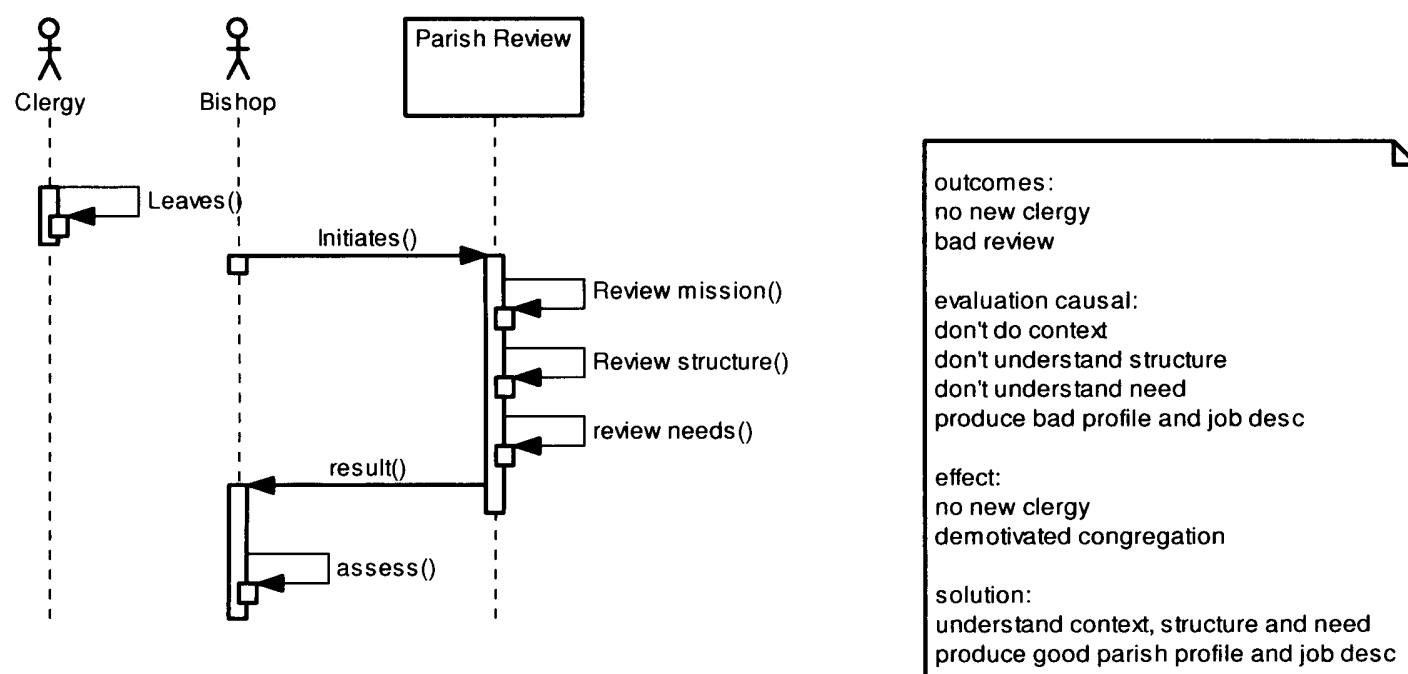

Figure 7-20 - Causal evaluation

There are two possible outcomes from the Bishops assessment at the end of the review shown in Figure 7-20 this could be a simple yes, no. Yes a position will be made available, no, a position will not be made available. It is the negative outcome which is of most concern.

\subsubsection{Effect evaluation}

The effect of no new clergy being appointed could mean a loss of resource creating; an inability to reach and service all areas of the parish; over stretching of existing resources. This may mean that some needs are not 
catered for.

\subsubsection{Treating the risk of not getting new clergy members}

There are two options which the parish has do nothing or do something. Doing nothing would mean that the bishop would arrange the review and provide an assessment based on it. The option to do something would include pro-active engagement of the parish in the review, involving as many people from and related to the parish as possible rather than only existing clergy.

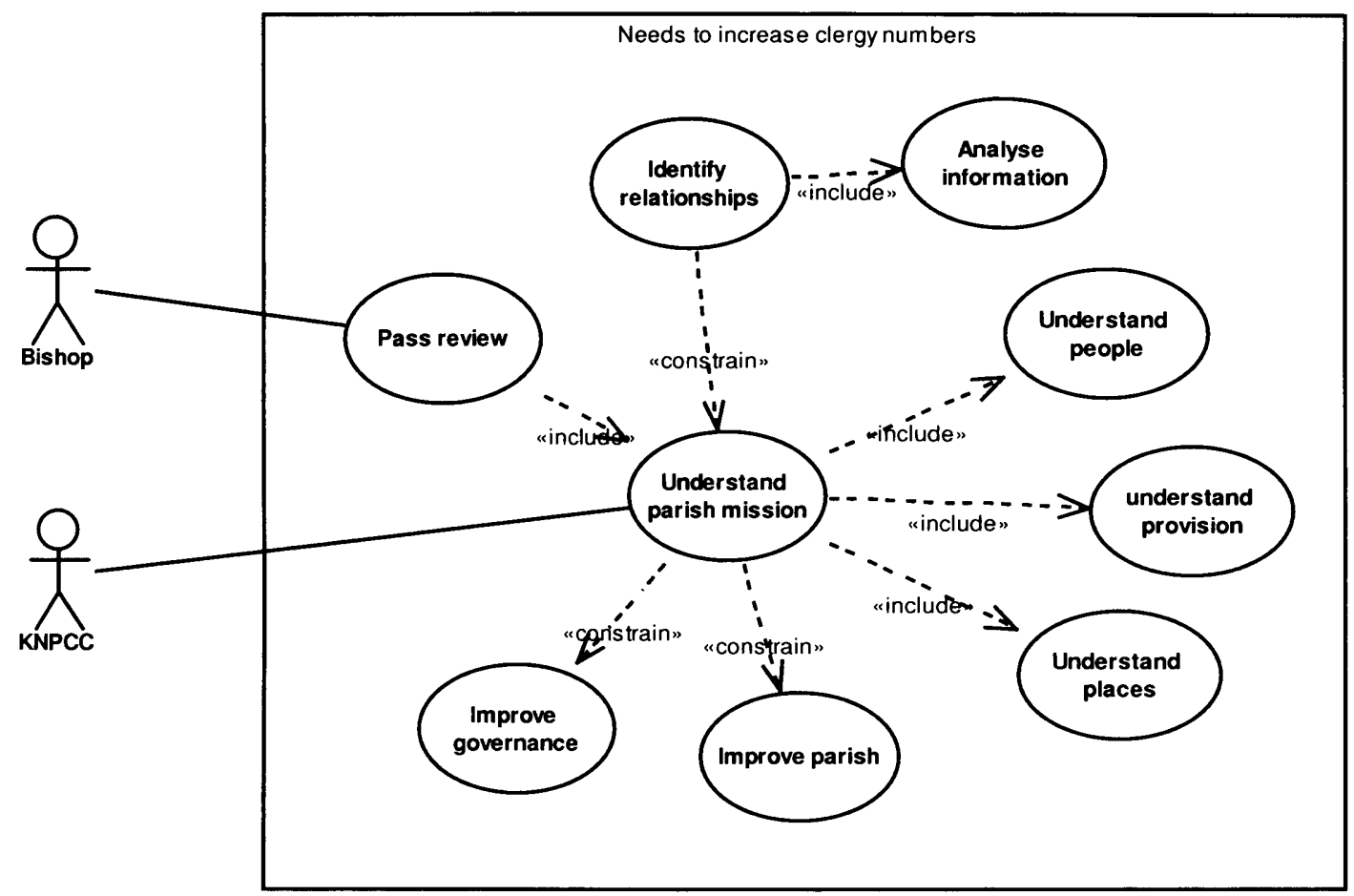

Figure 7-21 - Risk Treatment - No new clergy

This diagram, Figure 7-21, defines the needs of the team review, this is also the information required to fill in much of the missing detail in the system and context models. In fact it becomes obvious that the needs of the parish review are similar to the initial needs of risk management. This can be seen by the similarity between the terms used in the contexts and the terms used in the needs shown in the diagram above.

Based on the needs of the review scenarios can be developed providing ways to fulfil the needs described. These scenarios can have further detail added to them enabling them to be used as project plans. 


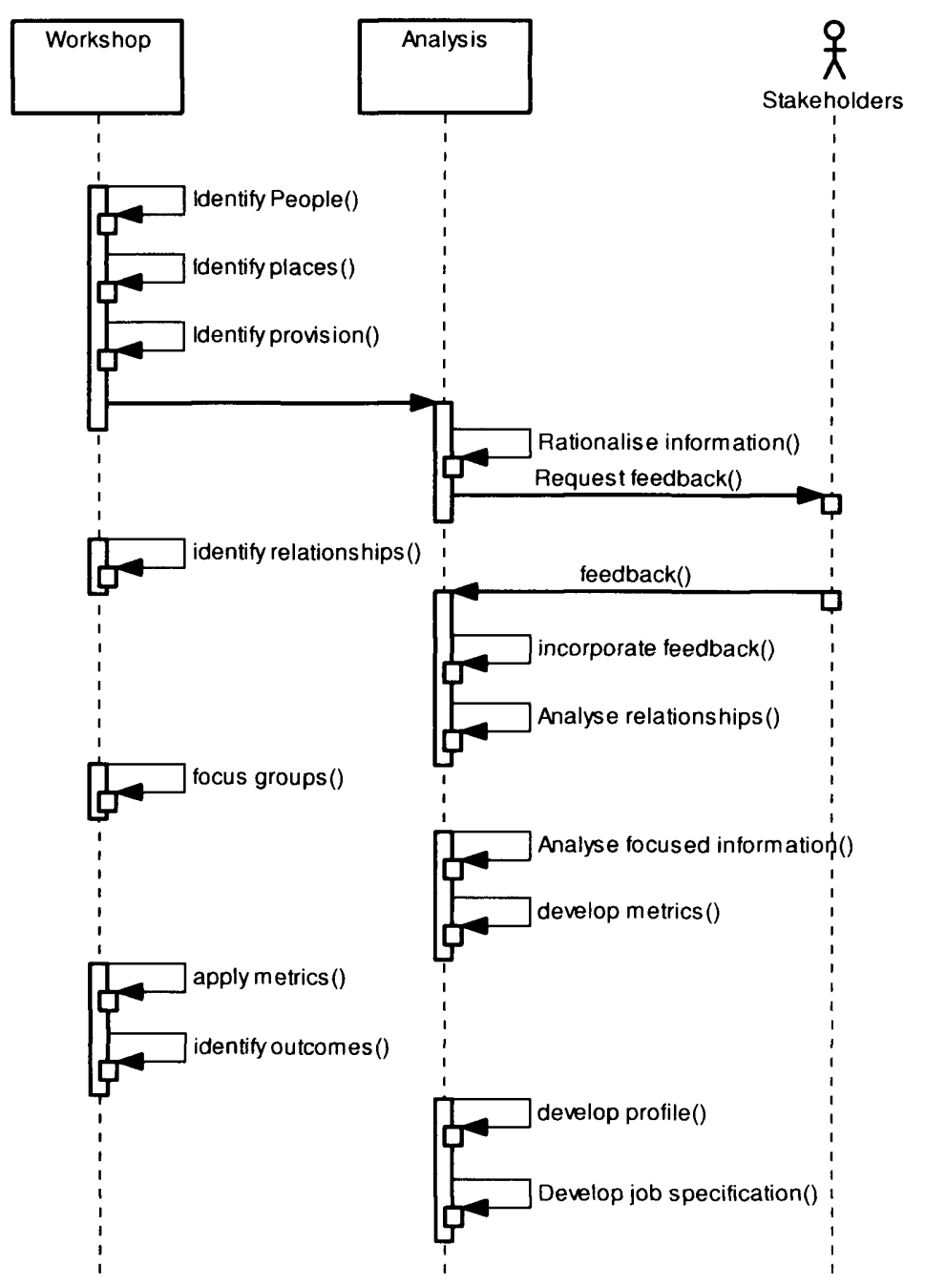

Figure 7-22 - Risk Treatment - No new clergy

The main approach to gathering information in the initial part of this work is to be completed through a number of workshops (Figure 7-22), the work uses workshops to ensure 'buy-in' providing stakeholders with the opportunity to input to the work and therefore have an emotional tie to the future of the work. This work is now complete and the information gathered is of great importance to the parish. The importance is not only in securing clergy resource but in the ability of the parish to understand and manage its structure, information and risk in a rigorous and timely fashion. 


\subsection{Conclusions}

These examples have shown some of the paths through this extensive and on-going case study. As this is real and ongoing work there are many commonly seen issues which arise which would be impossible to deal with accurately and consistently without the aid of processes and ontology.

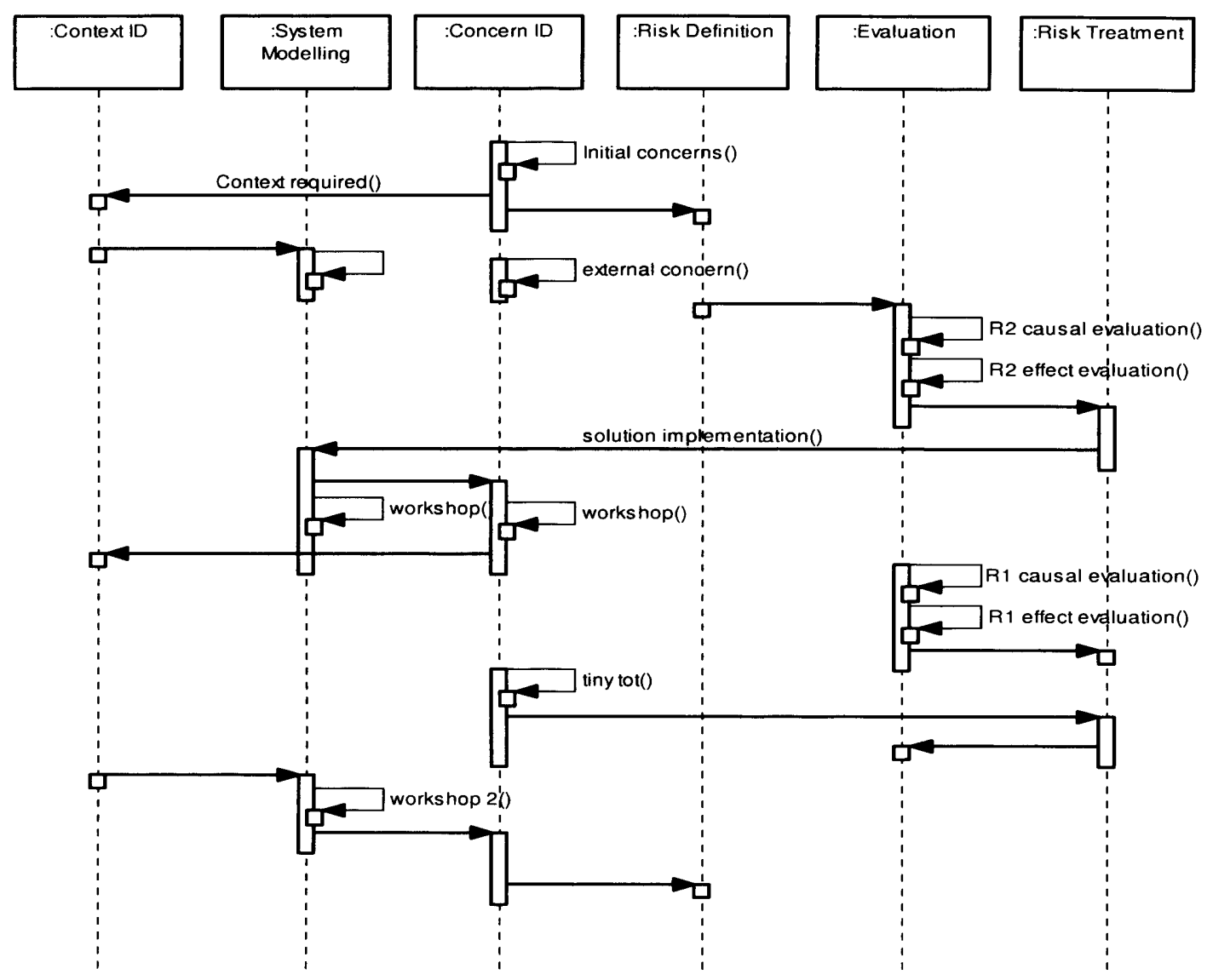

Figure 7-23 - Project actual Process Instance View

The sheer complexity of this plan, Figure 7-23, showing the execution of the processes from only the initial set of work emphasises the fact that without a formalised approach the management of risk, risk management would fall into disarray providing a stumbling block an inhibitor rather than the enabler that it 
should be.

\subsubsection{Evidence collection studies}

A number of evidence collection studies were conceived, three of which were completed during the investigative stages of this work. This section provides an overview of two of the case studies carried out during the development and verification of this work. Overviews are provided for the 'Project risk assessment' and 'Competence assessment and professional development' studies.

The final study focused on the application of the CORAS IT Security tool where it investigated the transferability of the tool to other areas of risk rather than the terminology or process for risk management. Details regarding this study can be found in Appendix A.

The two studies described comprise of an introduction giving an overview and background, the work carried out in the study (including relevant documentation from the study) and conclusions of each study providing a summary of its contribution.

In addition to the studies described in this chapter the principles within this work have been applied within a number of situations. In some cases this has supported and improved the work of specific companies within the rail and defence sectors. In others cases it has been found that the object oriented nature of the techniques worked against the culture within organisation meaning that a significant cultural change would be needed before a study could be carried out. 


\section{Study 1 - Project Risk Assessment}

This case study investigates the risks involved in accepting or tendering for projects, this includes considering cost, time, resource and reputation gained and lost due to working with specific clients.

\section{Assessment Context}

To understand the scope of the assessment the project and business contexts have been considered.

With no specific project context in place the generic start point discussed in Chapter 2 has been used and tailored to be relevant to this assessment.

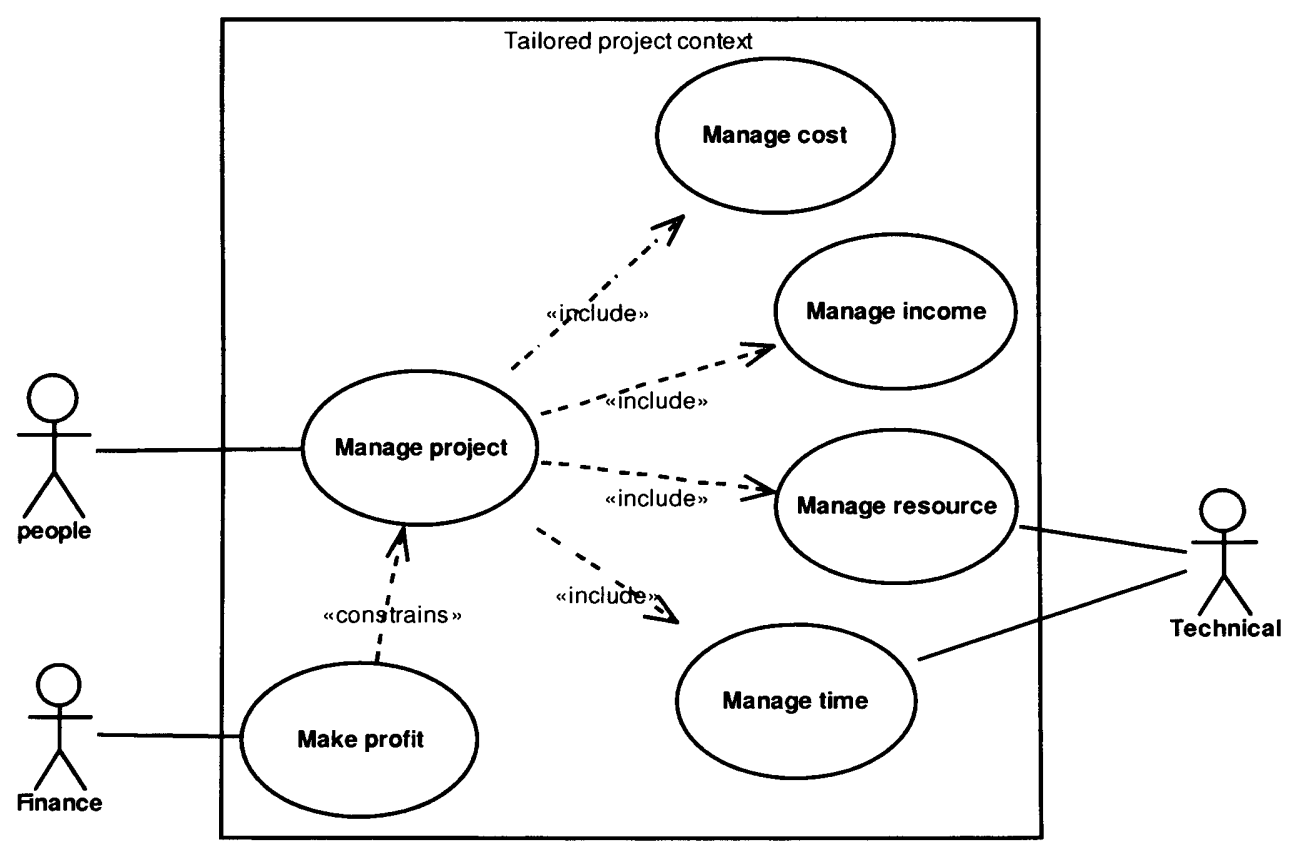

Figure 7-24 - Tailored project context

Only minor adjustments to the context have been made to the diagram in Figure 7-24. The requirement of make profit has been added, this requirement will constrain the assessment ensuring that the final output is related to profit.

The organisations development providing time and cost savings. The existence of the business context means that only minor alterations have been made. 


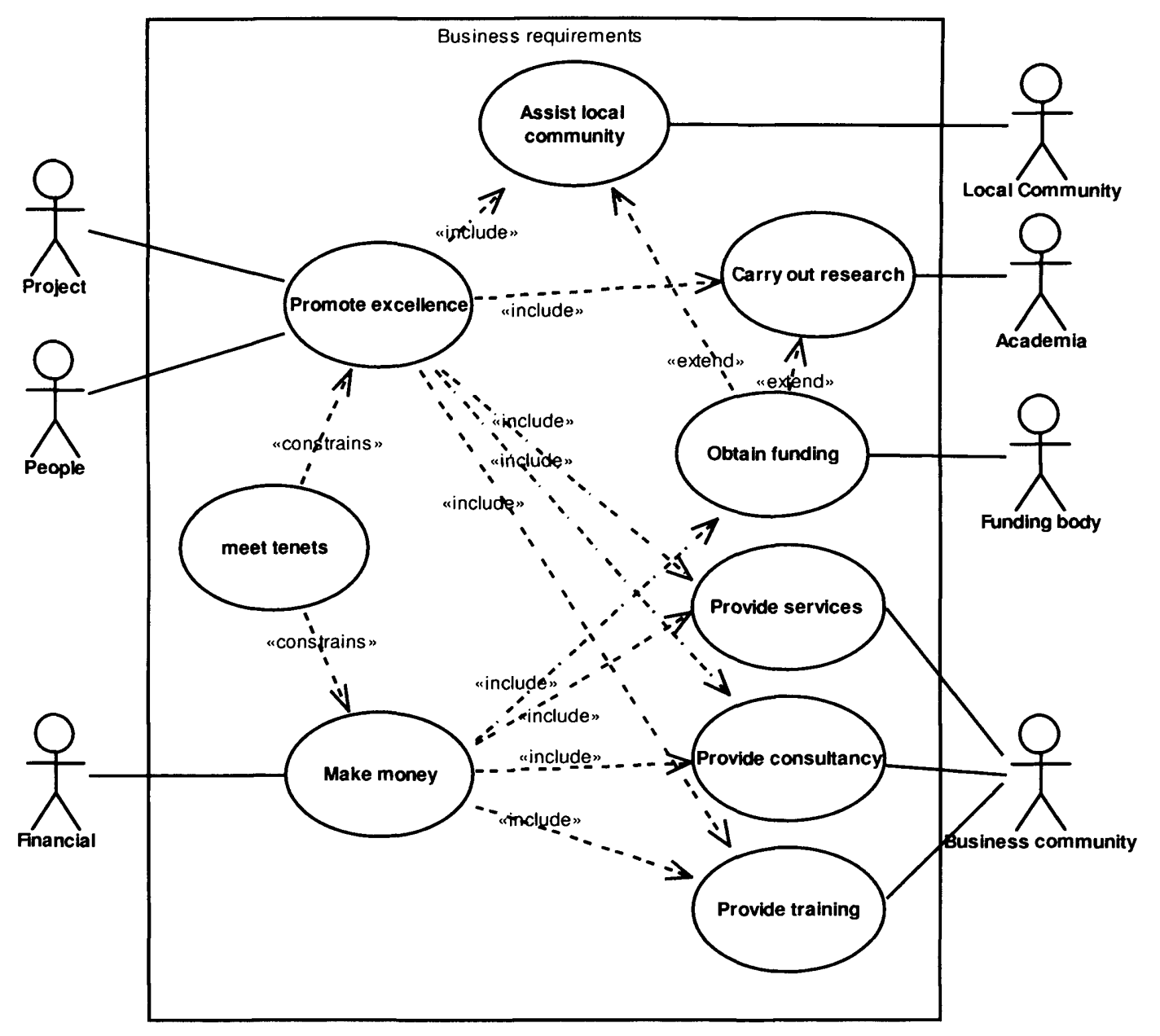

Figure 7-25 - Tailored business context

The minor alterations to Figure 7-25 relate to the association of contexts specifically the project context must be seen here. The people and financial contexts provide a completeness and support the need to further define these contexts as and when required.

The system view provides an understanding of the structures in place within the organisation relating to the assessment of project risk. 


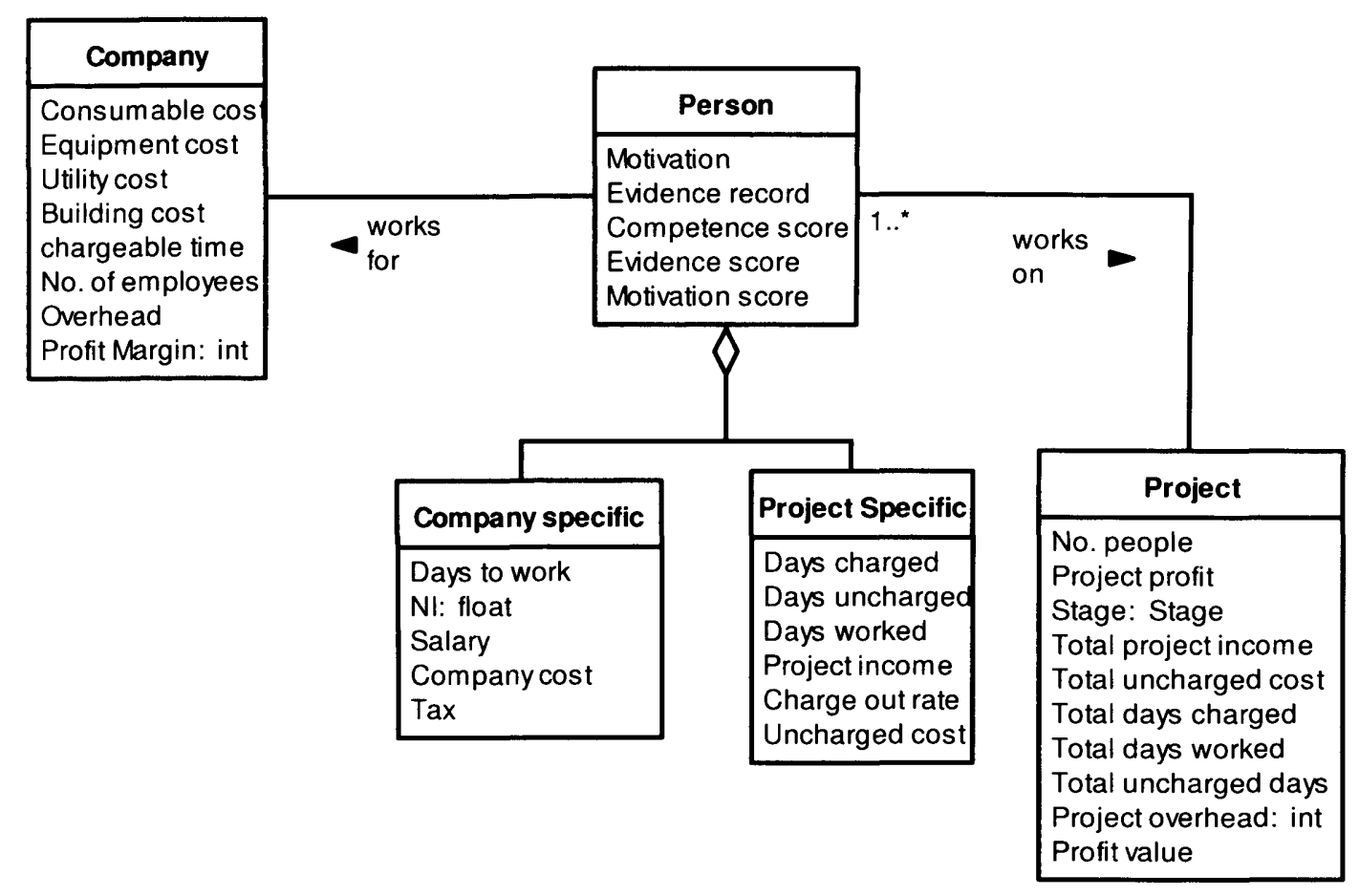

Figure 7-26 - System view

The system structure is taken from the company enterprise architecture which provides all of the information regarding the structure and processes associated with running of the organisation. The diagram in Figure 7-26 is a extract from the enterprise architecture which has been further developed to ensure that all relevant information from the assessment to be carried out can be collated and retained.

The constraint view provides the mathematical operators and their associated variables and formulas for implementation in the network 


\begin{tabular}{|c|c|c|c|}
\hline \multirow{4}{*}{ 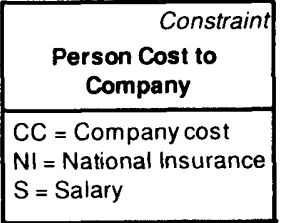 } & \multirow{3}{*}{\begin{tabular}{|l|}
\multicolumn{1}{|c|}{ Project Revenue } \\
PI - Project Income \\
PPI - Person income/project \\
NP - Number of People on project
\end{tabular}} & \begin{tabular}{r|} 
Constraint \\
Project Worth
\end{tabular} & \multirow{2}{*}{ Profit achieved } \\
\hline & & \multirow{2}{*}{$\begin{array}{l}\text { PI - Project Income } \\
\text { PP - Project Profit } \\
\text { PO - Project Overhead }\end{array}$} & \\
\hline & & & \multirow{3}{*}{$\begin{array}{l}\text { PO-Project Overhead } \\
\text { PM- Profit Margin } \\
\text { PP Project Profit } \\
\text { V- Value }\end{array}$} \\
\hline & \multirow[t]{2}{*}{ For $\{\mathrm{NP}\} \mathrm{PI}=\mathrm{PI}+\mathrm{PPI}()$ : void } & \multirow[t]{2}{*}{$P P=P I \cdot P O():$ void } & \\
\hline $\mathrm{CC}=\mathrm{S}+\mathrm{NI}()$ & & & \\
\hline $\begin{array}{l}\text { Constraint } \\
\text { Person cost to client }\end{array}$ & $\begin{array}{l}\text { Constraint } \\
\text { Project Cost to company }\end{array}$ & & \multirow{5}{*}{$\begin{array}{l}\text { IF } P P<0 \text { Then } V=\text { High Risk() } \\
\text { IF } P M \triangle P P>=0 \text { Then } V=\text { High Risk() } \\
\text { IF } P P=P M \text { Then } V=\text { Medium Risk() } \\
\text { IF } P P>P M \text { Then } V=\text { Low Risk() }\end{array}$} \\
\hline \multirow{2}{*}{$\begin{array}{l}\mathrm{DC}=\text { Days } \text { Charged } \\
\mathrm{PPI}=\text { Person income/project } \\
\mathrm{CR}=\text { Charge out Rate }\end{array}$} & \multirow{3}{*}{$\begin{array}{l}\text { CC - Company Cost } \\
\text { DW - Days worked } \\
\text { PO - Project Overhead } \\
\text { NP - Number of people on project }\end{array}$} & Company Profit & \\
\hline & & $\begin{array}{l}\text { PM - Profit Margin } \\
\text { PO - Project Overhead }\end{array}$ & \\
\hline \multirow{2}{*}{$P P I=D C * C R():$ void } & & $\mathrm{PM}=\mathrm{PO}^{*} 1.3():$ void & \\
\hline & For $\{N P\} P O=P O+C C^{*} D W():$ void & & \\
\hline
\end{tabular}

\section{Figure 7-27 - Constraint view}

The majority of the constraints defined in Figure 7-27 are addition and multiplication based operators. Profit achieved is slightly different, it provides an enumeration of the values calculated to this point. The enumeration gives a high, medium or low result as the final output of the risk assessment.

\section{Parametric view}

The parametric view enables the constraints defined above to be implemented as a network shown in Figure 7-28.

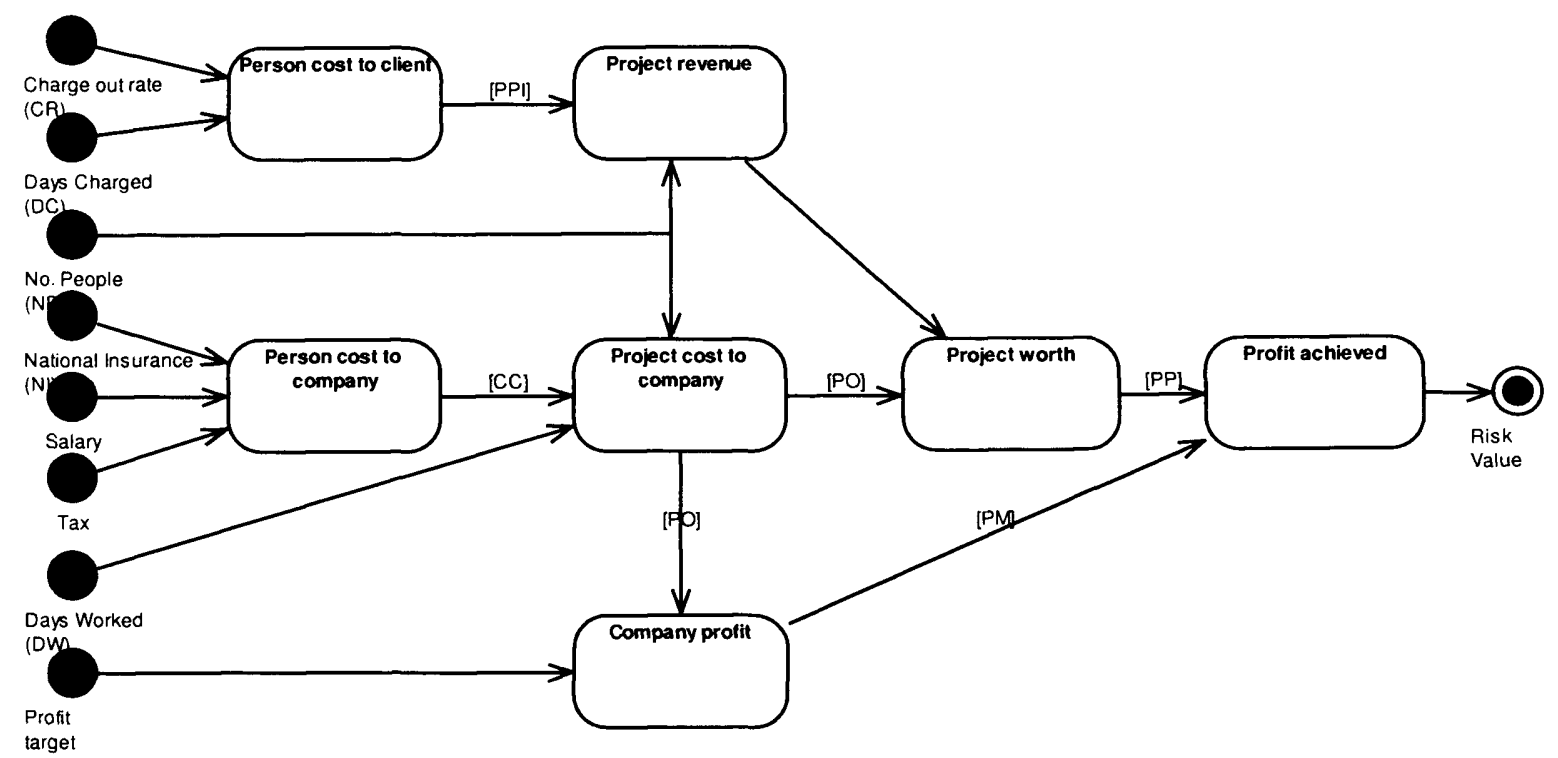

Figure 7-28 - Parametric view

Running the network with all the inputs present will provide an output of risk value from the network 


\section{Conclusions Study 1 - Project Risk Assessment}

This case study exercised the context identification and system development processes from the process set defined in Chapter 5. It also investigated the use of the SysML parametrics as an approach to developing bespoke risk assessments. The work on formalising risk assessments has been taken further since this case study was carried out. The latest status is discussed in the paper Formalising risk assessment through the use of SysML parametrics Brownsword and Perry (2009).

This study supported the need to identify relevant contexts within which an assessment will be applied. The study also highlighted the need to investigate the effect of the assessment on neighbouring contexts.

The study enabled further definition and clarification of the terms concern and risk and the need for separation between the concern identification and risk definition processes. 


\section{Study 2 - Competence assessment and professional development}

This case study investigates competence and professional development as an area to which the concepts of risk may be applied.

Competence and professional development are areas not normally associated with Risk or Risk Management. This case study shows that the same principles are used within competence assessment and risk assessment, the major difference being that the competence assessment looks for favourable outcomes i.e. how good an individual is, where as a risk assessment tends to look for flaws and failures, this ability to consider both sides of risk is discussed further by Hessami (1999) in Risk - A missed opportunity?

This study starts by understanding the need for the competence assessment within the organisation before looking at some of the features of the system.

\section{Assessment Context}

The first thoughts in this study were to define the need 'why carry out professional development', Professional development is one of the main reasons for carrying out competence assessments, In this case the need has been defined using a use case diagram, Figure 7-29.

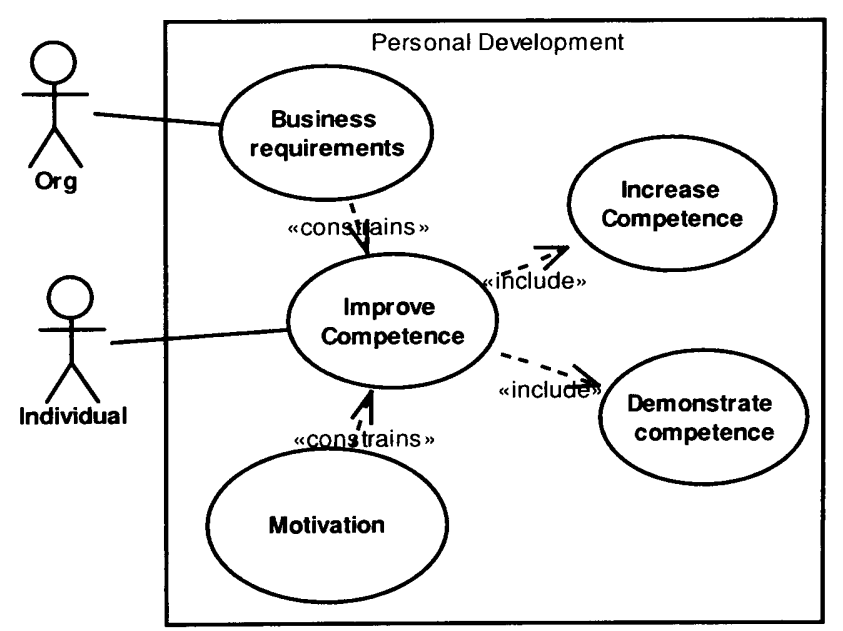

Figure 7-29 - Personal Development

The most important thing to understand when undertaking any work is 'why is this work being carried out'. The intention of the diagram above is to answer 
that question, in this case the work is being carried out to Improve competence of an Individual this is likely to be a member of staff, but may include students or the wider public.

It is important to know why the work is to be carried out however, this is rarely of any use without knowing what has to be done; again in this case competence must be both increased and demonstrated.

This has provided an understanding and record of the need for professional development and competence assessment to be carried out. This approach, using use cases to represent requirements or needs, has been used in many applications providing repeatability in the identification and recording of needs. Repeatability is one of the main concepts behind formalisation and as such this may provide a worthwhile approach to defining the needs of a risk assessment. The semantics behind the diagram above further support formalisation through the understanding of needs and contexts. 


\section{System}

Having the understanding of why the organisation wants to carry out professional development still does not explain how they are going to do it. This work started by analysing a number of competence frameworks and standards in order to understand the terminology and approaches taken by others to competence assessment.

Many frameworks and standards including ISM (now part of SFIA), SFIA and UK-SPEC follow similar approaches.

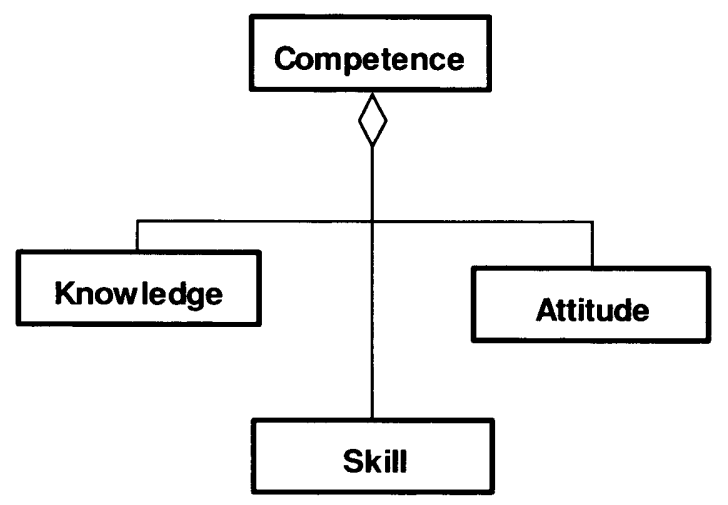

Figure 7-30 - Competence

Competence in Figure 7-30 has been defined as comprising of Knowledge, Skill and Attitude in many more recent cases the Attitude has been removed. Knowledge can be demonstrated through education, training and qualifications and provides the basis for competence. Skill is the persons understanding of their own domain and the way in which knowledge should be applied within it, Skills are often related to specific tools, techniques or products and as such can become a very long and daunting list. Attitude is the way a person approaches their work and relates it to moral and ethical standards.

This definition still leaves competence as a complex concept and difficult to repeatedly measure without gaining conflicting results. 


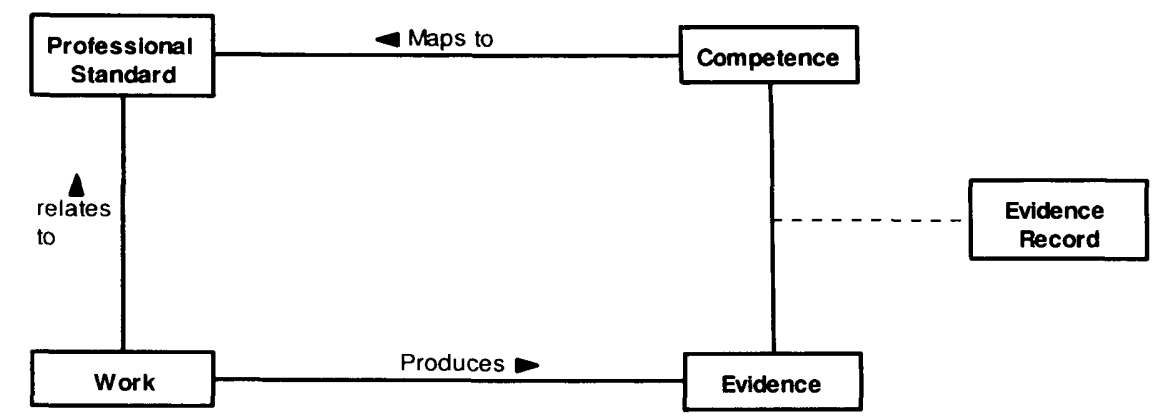

Figure 7-31 - Competence evidence

Figure 7-31 shows the generally accepted view of competence, that demonstration of competence is via the presentation of evidence. Evidence is to be based on work which has been carried out by the person under assessment. The difficultly in many cases is clearly relating evidence to the professional standard.

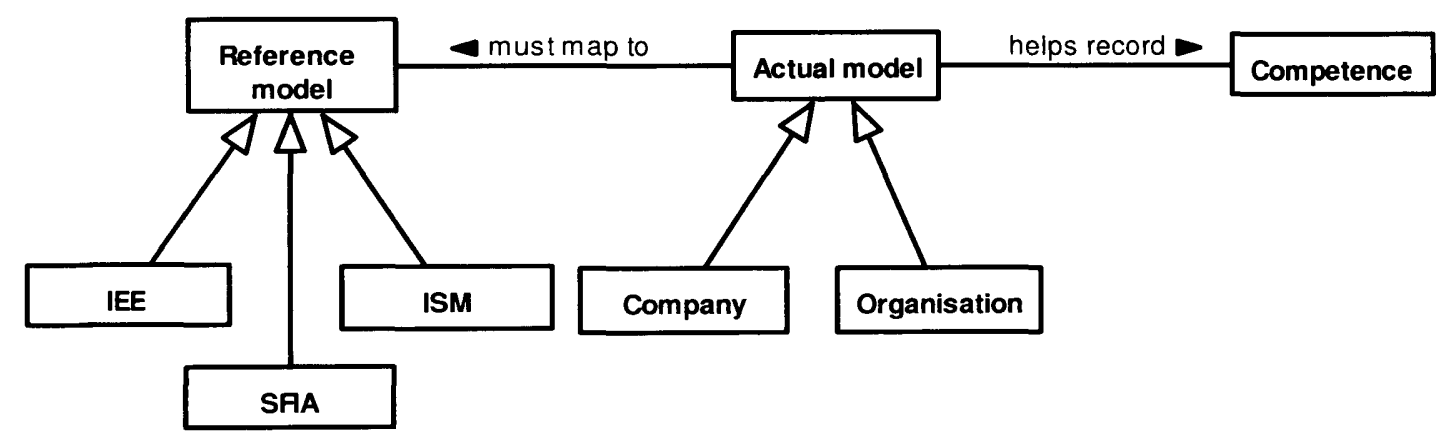

Figure 7-32 - standard - work - competence

To support the clear definition of evidence mapped to standards it is useful to have an organisational model that bridges the gap between work carried out and the competencies required for demonstration by the relevant standard. The diagram in Figure 7-32 proposes a high level view to support this idea showing that the organisational model supports the recording of competence against the professional standards by providing a mapping between the organisational competencies and the professional standard. 


\section{Conclusions Study 2 - Competence Assessment}

This study supports the concept that risk assessments are domain and application specific and that although many standard risk assessments from SWOT to Failure Modes and Effects Analysis (FMEA) exist it each has a specific purpose and should be used outside its scope with care. Competence assessment is a domain specific risk assessment for professional development.

The context and system diagrams set out the information required in the assessment of competence. It can be seen by the levelling system in place which is used to assess candidates that there is no space for lack or negative competence. This sets competence assessment apart from many risk assessments as it is focused on looking at how well someone can do their work rather than how badly or how likely they are to fail.

\subsubsection{Interpretation/Evaluation of Case studies}

There are a number of benefits which can be observed when using the UML within the case studies discussed. These benefits are based on the fundamental principles that the UML itself. The benefits focus on:

- 'views' ensuring that multiple contexts have been considered, these may include hierarchical, positional and procedural views.

- consistency ensuring that the knowledge and understanding which has been assimilated provides a consistent set across behavioural and static aspects. This consistency provides confidence that the 'views' have meaning and relevance.

- Integration - the UML with the SysML parametrics enables the reduction in the gap between contextual and scientific domains.

Many of the approaches and languages discussed in Chapter 4 provide consistency within their own domains, IDEF 3 for processes and IDEF 5 for ontologies for example, however they do enable clarity of checking across the boundaries of domains which the UML with its extensibility provides.

The use of the 7-views approach to process modelling has also provided benefits within the case studies. The strongest example of these benefits can 
be seen in the ability to re-organise or efficiently update the ordering of the processes when unforeseen circumstances arise. Obviously this ability to update the ordering of the processes would be much more complex had the processes been lacking in their definition. Without the knowledge of the relationships between artefacts and between artefacts and activities the time to re-order would be increased dramatically.

This work has focused on the contextual issues associated with the understanding of concerns and risks. The intention is not to suggest that specific tools such as FMEA, SWOT, FTA etc. should not be used. The intention is, however, to provide a framework to ensure that these detailed tools are applied in relevant and focused ways. There have also been a number of domains which have not been incorporated in detail within this work including Human Factors (HF) and project management. This is due to the body of knowledge which already exists in these areas. Again, it is the intention of this work to provide a framework to integrate with the current thinking in these areas.

\subsubsection{Summary}

These case studies have enabled the generation and understanding of knowledge regarding risk management. They have in this way aided in the definition of the processes, methodology and ontology defined by this work. The table below shows the UML constructs used within each study and identifies benefits provided in each case. 
Table 7-1 - Case Study Usage of UML

\begin{tabular}{|c|c|c|c|c|}
\hline UML construct & $\begin{array}{l}\text { Project Metrics } \\
\text { Use/benefit }\end{array}$ & $\begin{array}{l}\text { Competence } \\
\text { assessment } \\
\text { Use/benefit }\end{array}$ & $\begin{array}{l}\text { CORAS tool } \\
\text { (Appendix A) } \\
\text { Use/benefit }\end{array}$ & KNPCC \\
\hline Class diagram & $\begin{array}{l}\text { Clarity of } \\
\text { business structure } \\
\text { enabling } \\
\text { development of } \\
\text { metrics }\end{array}$ & $\begin{array}{l}\text { Clarity of system } \\
\text { structure } \\
\text { including } \\
\text { definitions }\end{array}$ & $\begin{array}{l}\text { Definition of } \\
\text { assessment scope } \\
\text { and visualisation } \\
\text { of stakeholders }\end{array}$ & $\begin{array}{l}\text { System concepts } \\
\text { definition, }\end{array}$ \\
\hline Object diagram & & & & $\begin{array}{l}\text { Issue and risk } \\
\text { capture }\end{array}$ \\
\hline $\begin{array}{l}\text { Deployment } \\
\text { diagram }\end{array}$ & & & & $\begin{array}{l}\text { Visualisation of } \\
\text { locations within } \\
\text { system }\end{array}$ \\
\hline $\begin{array}{l}\text { Component } \\
\text { diagram }\end{array}$ & & & & $\begin{array}{l}\text { Visualisation of } \\
\text { constructs within } \\
\text { system - without } \\
\text { location }\end{array}$ \\
\hline Use case diagram & $\begin{array}{l}\text { Clarity of reason } \\
\text { and scope for } \\
\text { carrying out } \\
\text { project } \\
\text { monitoring }\end{array}$ & $\begin{array}{l}\text { Clarity of context } \\
\text { and business } \\
\text { justification for } \\
\text { carrying out } \\
\text { competence } \\
\text { assessment }\end{array}$ & $\begin{array}{l}\text { Visualisation of } \\
\text { situation analysis } \\
\text { including } \\
\text { definition of } \\
\text { threats and } \\
\text { identification of } \\
\text { treatment }\end{array}$ & $\begin{array}{l}\text { Identification of } \\
\text { system and } \\
\text { assessment } \\
\text { contexts }\end{array}$ \\
\hline Sequence diagram & & & & $\begin{array}{l}\text { Visualisation of } \\
\text { cause and effect } \\
\text { scenarios }\end{array}$ \\
\hline $\begin{array}{l}\text { Parametric } \\
\text { diagrams }\end{array}$ & $\begin{array}{l}\text { Visualisation of } \\
\text { mathematical } \\
\text { operators and } \\
\text { formula }\end{array}$ & & & \\
\hline
\end{tabular}

Although there is value in using each of the diagram types identified in 
Table 7-1 individually, a more intangible yet valuable benefit is realised when the diagrams are used together to ensure consistency. This can be achieved by following the consistency checks set out within the UML specification. The multiple, holistic and consistent aspects of the UML provide unique benefit regarding the use of the UML rather than other approaches such as those discussed in Chapter 4. For clarity this is due to the lack of internal consistency between multiple views within many other techniques or loosely coupled approaches such as IDEF standards.

The objective of this chapter was to demonstrate the applicability of the processes and ontology defined in this work.

This has been demonstrated through the two paths discussed within this chapter; the first investigating an health and safety issue relating to scalding injuries of children and including wider issues into the risk definition and solution space in a timely fashion based on the information available; the second investigated governance and resource issues which has prompted an information gathering exercise and review of the whole organisation.

The availability and application of the processes and ontology have been shown to benefit the organisations through improved understanding and communication through formalisation, this in turn has enabled the identification of relevant concerns and will provide more efficient evaluation of future risks.

Outside this work the principles defined within are continually applied within Brass Bullet Ltd. supporting project and business development. They have also been applied by the author within governmental and professional bodies and engineering organisations within the rail and defence sectors. 


\section{Conclusions}

\subsection{Introduction}

Chapter six described two threads through one of the case studies carried out during this work, it concluded that the processes and ontology defined by the work could be used in a pragmatic way to provide a formalised approach to the management of risk. This chapter collates the conclusions from each chapter and shows where each of the overall objectives of the work have been meet. It also defines further work which could be carried out to further develop this work.

\subsection{Case study evaluation}

The case study supports the overall objective of this work. It shows the benefits of using a formalised, pragmatic approach to risk management. This is highlighted by the ability to change the methodology in a pragmatic way without losing control of the risk management activities or association with the items within the study.

The study applied all of the processes defined within chapter 6 with negligible issues in the application. Issues with the use of these processes and ontology are related to the culture of the applying organisation rather than technical issues with the activities and artefacts within the processes. The cultural issues include a lack of basic understanding of the terminology to be used, a lack of ability to abstract away from details and the inability to be open to a different way of working.

In the main case study the application of the processes defined in Chapter 6 And ontology defined in Chapter 5 enabled the organisation to understand and prioritise concerns before establishing risks vastly reducing the number and complexity of the risks faced.

Limitations with this work include:

- The cultural change required in many organisations to use a pragmatic methodology for risk 
- The perceived lack of application of the processes and ontology to engineering and business applications due to the main study not directly residing in one of these domains.

It is reasonable to generalise the results of this work suggesting that the processes and ontology defined within will apply to engineering and business based organisations. This ability to generalise is based on the observations made within engineering organisations where the principles behind these processes have been applied and the relationship between the processes and ontology and the international standards which are already general in nature.

\subsection{Summary of Chapters}

Chapter 1 presented the aims and objectives of this thesis detailing the contribution of each chapter.

Chapter 2 presented issues with the risk management process and the underlying terminology associated with risk management which exists in general literature and standards. The chapter identifies the need for a formalised approach to the processes and terminology of risk. It also concludes that as tools and techniques are for the most part industry and application specific therefore the detailed implementation of risk assessment would not be considered by the rest of this work.

Chapter 3 presented the methodology for the research and recognises the need for a phenomenological perspective and identifies issues with using a phenomenological in a generally positivist domain.

Chapter 4 discussed the requirements for a model based framework to formalise risk management and proposed the Unified Modelling Language $(\mathrm{UML})$ as a language capable of delivering this formalisation. Formalisation in this work ensures that consistency, repeatability, multiple views and pragmatic application are all considered. This chapter identifies the UML along with the Systems Modelling Language (SysML) profile of the UML as the only tool, 
from those investigated by this work, to fulfil all of the requirements defined. The tools considered exhibited two main weaknesses, either their focus was on static or behavioural modelling or a lack of consistency was identified meaning that consistency checking between ontology and process views would become increasingly complex.

Chapter 5 defined risk and presented an ontology for risk management incorporating the definitions and relationships for terminology which is often confused and mis-used. The ontology of any industry is fundamental. Without a clear understanding of the terms used within the domain and the relationships between those terms it is difficult to clearly communicate within the domain and almost impossible to teach. This ontology provides the ability to improve the teaching of risk management at a grass routes level.

Chapter 6 defined the processes to be use when implementing risk management. The chapter presented requirements, behaviour and information views of the risk management processes, ensuring that multiple views can be considered and consistency check when applying the processes. The use of object orientation ensures that the processes support a multi-view, consistent application whilst modularisation ensures that they can be used in a pragmatic manner. Following the definition of the processes theoretical and pragmatic methodologies for their application have been defined, the pragmatic methodology is based on the use of the processes within case studies.

Chapter 7 demonstrated the application of the processes and ontology through the presentation of two threads within one of the case studies completed during the execution of this work. These threads show the applicability of the processes and ontology to organisational, project and health and safety issues. 


\subsection{Conclusions by Objective}

The primary aim of this thesis was to develop a formalised approach to the management of risk using a model based approach. This addresses the lack of formalisation across risk management in many industries, applications and education.

To achieve the overall aim both terminology and processes related to risk have been understood and formalised. Formalised ontology and processes have been developed and demonstrated through a number of threads abstracted from a case study.

This section maps the main conclusions from each chapter to the Objectives set out in Chapter 1. It explains where each has been defined and details why the cases study threads show that these definitions, approach and methodology provide a formalised approach to the management of risk.

- Chapter 2 - the purpose of which was to review and understand the terminology and processes related to risk management. Has presented a view of risk management which has at a high level been unchanged for many years. The changes that have been suggested and made in this latent period have confused the terminology and process further. This chapter has highlighted the process confusion and issues with terminology enabling an improved definition to be made.

- Chapter 4 - the purpose of which was to discuss the requirements for a model based framework for the formalisation of risk management and propose a framework which fulfils the requirements. Has identified and justified a formalisation tool which is not only relevant to risk management but to all areas requiring formalisation of ontology and process.

- Chapter 5 - the purpose of which was to define risk and present an ontology for risk management. Has presented a clear concise candidate definition for risk and shown the ontology in which this definition resides. This provides a clear set of terminology enabling improvement of the implementation and teaching of risk management. 
- Chapter 6 - the purpose of which was to define the processes required to carry out risk management using a multi-view approach. Has presented multiple views of the key processes required to carry out risk management. These views have provided a modular, consistent and multi-view approach to risk management.

- Chapter 7 - the purpose of which was to demonstrate through application the applicability of the processes and ontology. Has shown the application of the processes and ontology to business and health and safety issues. This has enabled the improved management of risk within the organisation under consideration.

\subsection{Contributions}

The individual contributions made by this work are:

- Provision of independent interpretations and analysis of international standards related to risk management.

- Provision of improved access to relevant areas of international standards and frameworks through the overview models described.

- Provision of a comparison of description languages for formalisation.

- Provision of a related view of the terminology of risk management.

- Provision of a set of risk management processes, including their behaviour, requirements and artefacts using the seven-views approach.

- Provision of a pragmatic methodology for processes application enabling pro-active management of change.

- Provision of a worked example approach to the application of the processes and ontology through the case study. 


\subsection{Further work}

This section defines a number of opportunities for further work within academic and industrial arenas.

\subsubsection{Academic}

It is expected that industry and application specific ontologies will be defined and mapped to the ontology within this work. This will provide an improved communications medium for those working in different sectors ensuring that consistent meaning is interpreted when different words are used. The ontology within this work may also be updated specifically with reference to the use of the term 'Hazard' as it is not yet clear as to whether a hazard is a risk but from another stakeholders perspective or if it is a cause of an outcome. There is a specific need for further research into the generic concepts and definitions of hazards with the purpose of enabling a clear relationship to be drawn between hazards and risks.

With so many authors defining tailored risk assessments a formalised approach to the definition of bespoke risk assessments to complement the risk management approach defined within this work would is required. Such an approach should provide improved re-use of existing assessments, improved understanding of the relationship to the system under consideration and clear traceability between bespoke and generic risk assessments. This may include the modelling and tracing of existing risk assessments providing a clear understanding of how they related to the source and product variables with which they are concerned.

The use of a formalised approach to risk should support the definition of complete sets of risks for products and systems. However there is a complexity in the verification of completeness of these sets of risk. The application of an additional tool such as metrics to the risk set to give confidence merits investigation. 


\subsubsection{Industrial}

The processes and ontology defined within this work are regularly applied within Brass Bullet Ltd and the Kings Norton PCC. This approach is adding value to the management of financial, organisational and project risk within these organisations. Interest has been shown by the IET and NCC in applying the processes and ontology to the Enterprise architecture and Systems Engineering project which they are undertaking. This is will investigate the use of the processes within the change management aspects of enterprise architecture. 


\section{References}

Standards Australia and Standards New Zealand (1999), AS/NZS 4360 Risk Management

Standards Australia and Standards New Zealand (2004), AS/NZS 4360 Risk Management

Adhitya, A., Srinivasan, R., Karim, I.A. (2007), A model based rescheduling framework for managing abnormal supply chain events, Computers and Chemical Engineering Vol. 31 pp. 496-518

Aggarwal, P. and Ganeshan, R. (2007), Using risk management tools on B2Bs: An exploratory investigation, Int. J. Production Economics vol. 108 pp 2-7

Ale, B.J.M. (2005), Living with risk: a management question, Reliability Engineering and System Safety Vol. 90 pp 196-205

Aven, T. (2003), Foundations of Risk Analysis: A Knowledge and Decisionoriented Perspective, WileyBlackwell

Aven, T. and Kristensen, V. (2005), Perspectives on risk: review and discussion of the basis for establishing a unified and holistic approach, Reliability Engineering and System Safety Vol. 90 pp 1-14

British Standards Institution (1996), BS 8444-3 Risk Management - Part 3: Guide to risk analysis of technical systems.

British Standards Institution (2000), BS 6079-3:2000 Project management - Part 3: Guide to the management of business related project risk 
Baclawski, K., Kokar, M., Kogut, P., Hart, L., Smith, J., Holmes, W., Letkowski, J. and Aronson, M. (2001), Extending UML to support ontology engineering for the Semantic Web. In Fourth International Conference on the Unified Modeling Language, M. Gogolla, C. Kobryn (Ed), pages 342360. Lecture Notes in Computer Science 2185:342-360. Springer-Verlag.

Barrow, R. (2005), Setting New Boundaries - Applying the UML to Railway Standards, The IEE Seminar on: UML for Systems Engineers

Bayley, C. (2004), Modelling Interlocking Systems with UML, IEE System Safety Conference

Belzer, R.B. (2001), Getting beyond 'grin and bear it' in the practice of risk management, Reliability engineering and system safety

BLOOM, B.S. (ed.) (1956), Taxonomy of Educational Objectives, the classification of educational goals - Handbook I: Cognitive Domain New York: McKay

Boehm, B.W. (1988), A Spiral Model of Software Development and Enhancement, IEEE Computer

Boehm, B.W. (1989), Tutorial: Software Risk Management, IEEE Computer

Boiss, A.C. (2005), Risk Analysis and Risk Management using UML, MSc Case Study, Cardiff University

den Braber, F., Lund, M. S., Stølen, K., Vraalsen, F. (2005), Integrating security in the development process with UML, in MehdiKhosrow-Pour (ed), Encyclopaedia of Information Science and Technologypages pp. 1560-1566 
den Braber, F., Mildal, A.-B., Nes, J., Stølen, K., Vraalsen, F. (2006), Experiences from Using the CORAS Methodology to Analyze a Web Application, Journal of Cases on Information Technology

Broendeland, G. and Stolen, K. (2004), Using risk analysis to assess user trust -a net-bank scenario, Second International Conference on Trust Management (iTrust'04)

Brownsword, M., Setchi, R. (2007), Putting Risk into Context, IET system safety conf

Brownsword, M., Perry, S. (2009), Formalising risk assessment through the use of SysML parametrics, INCOSE

Brownsword, M., Setchi, R. (2005), Picturing Risk - Connecting Industries, The IEE Seminar on: UML for Systems Engineers

CENELEC (1999), EN 50126: Railway applications - The specification and demonstration of Reliability, Availability, Maintainability and Safety (RAMS)

CIPS (2007), Risk Management Practice Guideline

Cacciabue, P.C. (2005), Human error risk management methodology for safety audit of a large railway organisation, Applied Ergonomics Vol $36 \mathrm{pp}$ 709-718

Carr, M.J., Konda, S.L., Monarch, I., Ulrich, F. (1993), Taxonomy-Based Risk Identification, SEI Carnegie Mellon University

Clarke, L. (2007), Thinking possibilistically in a probabilistic world, Significance statistics making sense, Royal statistical society 
Cranefield, S. and Purvis, M. (1999), UML as an ontology modelling language, CEUR

Dalla Valle, L. and Giudici, P. (2008), A Bayesian approach to estimate the marginal loss distributions in operational risk management, Computational Statistics \& Data Analyssis Vol. 52 pp 3107-3127

Davis, J.R., Fogarty, J.A. and Richard, E.E. (2008), Human health and performance risk management an approach for exploration missions, Aeta Astronautica Vol.63 pp. 988-995

Delvosalle, C., Fievez, C., Pipart, A., Casal Fabreg, J., Planas, E., Christou, M., Mushtaq, F. (2005), Identification of reference accident scenarios in SEVESO establishments, Reliability Engineering and System Safety Vol. 90 pp. 238-246

Dikmen, I., Birgonul, M.T., Anac, C., Tah, J.H.M. and Aouad, G. (2008), Learning from risks: A tool for post project risk assessment, Automation in Construction Vol.18 pp. 42-50

ANSI/EIA (1999), EIA 632: Processes for Engineering a System.

Faith, N. (2000), Derail: Why Trains Crash, Channel 4 Books

Flage, R. and Aven, T. (2009), On treatment of uncertainty in system planning, Reliability Engineering and System Safety Vol. 94 pp. 884-890

Gamper, C.D. and Turcanu, C. (2009), Can public participation help managing risks from natural hazards?, Safety Science Vol. 47 pp. 522-528

Giralt, C. (2002), Petri Nets for Systems Engineering, Springer 
Haplin, T. and Morgan, T. (2008), Information Modeling and Relational Databases, Morgan Kaufmann

Han, S.H., Kim, D.Y., Kim, H., Jang, W. (2008), A web based integrated system for international project risk management, Automation in Construction Vol. 17 pp.342-356

Harvey C.R. (Undated), Campbell R. Harvey's Hypertextual Finance Glossary, http://www.duke.edu/ charvey

Electronically accessed 22 April 2009

Hess, J. and Bernard, O. (2008), Design and study of a risk management criterion for an-unstable anaerobic wastewater treatment process, Journal of Process control Vol. 18 pp. 71-79

Hessami A. (1999), Risk - A Missed Opportunity?, Risk and Continuity Vol. 2 pp. $17-26$

Hollnagel, E. (2008), Risk + Barriers = Safety?, Safety Science Vol. 46 pp. 221-229

Holt, J.D. (2004), Those who can - use ISO IEC 15288, INCOSE Spring conference

Holt, J.D. (2005), A Pragmatic Guide to Business Process Modelling, BCS

Holt, J.D. (2007), UML for Systems Engineering: watching the wheels

Holt, J.D. (2009), Enterprise architecture - systems engineering in the mist, INCOSE

Hughes, B., Cotterell, M. (1999), Software Project Management, McGrawHill 
Hunt-Sturman, A. and Jackson, N. (2009), Development and evaluation of a risk management methodology for pedestrian surfaces, Safety Science Vol. 47 pp. 131-137

ICAEW (2002), Risk management for SMEs

Knowledge Based Systems, Inc. (1994), IDEF5 Method Report

Knowledge Based Systems, Inc. (1995), IDEF3 Process description capture method report

IEC (1998), IEC 61508 Functional safety of electrical/electronic/programmable electronic safety-related systems

IEEE (1998), IEEE Std 1220-1998 IEEE Standard for Application and Management of the Systems Engineering Process -Description

INCOSE (1996), Systems Engineering Capability Assessment Model

INCOSE (2007), Systems Engineering Handbook

IRM, AIRMIC \& ALARM (2002), A Risk Management Standard

ISO (1985), ISO 5807-1985, Information processing - Documentation symbols and conventions for data, program and system flowcharts, program network charts and system resources charts

ISO (2002), BS ISO 15288:2002 Systems engineering - System life cycle processes 
ISO (2004), ISO 10303-11 Industrial automation systems and integration -Product data representation and exchange -- Part 11: Description methods: The EXPRESS language reference manual

ISO (2006), ISO 16085 Systems and software engineering - Life cycle processes - Risk management

ISO (2007), ISO/IEC CD Guide 73 Risk Management - Vocabulary

ISO (2008a), BS ISO 31000 Risk Management - Principles and guidelines on implementation

ISO (2008b), ISO 31010: Risk Management - Risk Assessment Techniques

ITU-T (1999), Specification and description language

Jacobson, I., Booch, G. and Rumbaugh, J. (1999), The Unified Software Development Process, Addison-Wesley Professional

Jenkins, R.E., Brown, R.D.H., Phillips, M.R. (2009), Harbour porpoise (Phocoena phocoena) conservation management: A dimensional approach, Marine Policy

Kaplan, R. S. and Norton, D. P. (1992), The Balanced scorecard: measures that drive performance, Harvard Business Review

Kirchsteiger, C. (2008), Carbon capture and storage desirability from a risk management point of view, Safety Science Vol. 46 pp. 1149-1154

Kirstensen, V., Aven, T., Ford, D. (2006), A new perspective on Renn and Klinke's approach to risk evaluation and management, Reliability Engineering and System Safety Vol. 91 pp. 421-432 
Krogstie, J. (2008), Using EEML for combined goal and process oriented modeling: A case study, Proceeedings of EMMSAD

Lee, E., Park, Y., Shin, J.G. (2009), Large engineering project risk management using a Bayesian belief network, Expert Systems with Applications Vol. 36 pp. 5880-5887

Leveson, N.G. (1995), Safeware, system safety and computers, AddisonWeslyey Professional

Lund, M., Braber, F., Stolen, K., Vraasen, F. (2004), A UML profile for the identification and analysis of security risks during structured brainstorming, Sintef ICT

MOD (2007), The MOD Architecture Framework (MODAF) Version 1.1

Mazouni, M. and Aubry, J. (2007), A PHA based on a systemic and generic ontology, IEEE/INFORMS International Conference on Service Operation and Logistics and Informatics

McConnell, S. (1996), Software Quality at Top Speed, Software Development Vol. 4 pp. 38-42

McNeillis, P. (2005), Cognitive Mapping and UML Modelling Comparing Book and Mind, The IEE Seminar on: UML for Systems Engineers

Miller, G. (1956), The Magical Number Seven, Plus or Minus Two: Some Limits on Our Capacity for Processing Information, The Psychological review 
Mohaghegh, Z., Kazemi, R. and Mosleh, A. (2009), Incorporating organizational factors into Probabilistic Risk Assessment (PRA) of complex socio-technical systems: A hybrid technique formalization, Reliability Engineering and System Safety Vol. 94 pp. 1000-1018

NATO (1997), NATO quality assurance requirements for software development AQAP-150

Neiger, D., Rotaru, K., Churilov, L. (2009) Supply chain risk identification with value-focused process engineering, Journal of Operations Management Vol. 27 pp.154-168

Nicola, A., Kabilan, V., Missikoff, M. and Mojtahed, V., (2007), Practical Issues in Ontology Modeling: The Case of Defence Conceptual Modeling Framework-Ontology, in Doumeingts et al, Enterprise Interoperability, Springer London

Nilsen, A.S. (2008), Tools for empowerment in local risk management, Safety Science Vol. 46 pp. 858-868

OMG (2007), OMG UML Specification

OMG (2008a), Business Process Modeling Notationm, V1.1

OMG (2008b), UML(TM) Profile for Modeling Quality of Service and Fault Tolerance Characteristics and Mechanisms Specification

OMG (2008c), OMG SysML Specification

Olsen, O.E. and Lindoe, P.H. (2008), Risk on the ramble: The international transfer of risk and vulnerability, Safety Science 
Peckham, J. and MacKellar, B. (2001), Generating code for engineering design systems using software patterns, Artifical Intelligence in Engineering Vol. 15 pp. 219-226

Perry, S. (2006), When is a Process Model Not a Process Model - A Comparison between UML and BPMN, IEE seminar on Process Modelling using UML

Pezzullo, L. and De Filippo, R. (2009), Perceptions of industrial risk and emergency management procedures in Hazmat Logistics: A qualitative mental model approach, Safety Science Vol. 47 pp. 537-541

Redmill, F. (2002), Risk analysis - a subjective process, Engineering Management Journal

Remenyi, D. (1998), Doing research in business and management, Sage Publications

Remenyi, D. and Heafield, A. (1996), Business process re-engineering: some aspects of how to evaluate and manage the risk exposure, International Journal of Project Management Vol. 14 pp. 349-357

Restrepo, C.E., Simonoff, J.S. and Zimmerman, R. (2008), Causes, cost consequences, and risk implications of accidents in US hazardous liquid pipeline infrastructure, International Journal of Critical Infrastructure Protection

Roland, H.E. and Moriarty, B. (1990), System Safety Engineering and Management, Wiley-IEEE

Royce, W. (1970), Managing the Development of Large Software Systems, Proceedings of IEEE WESCON 26 
Senge, P. (2006), The Fifth Discipline: The Art \& Practice of The Learning Organisation, Random House Business Books

Sheard, S.A. (1997), The Frameworks Quagmire, Crosstalk: The Journalof Defense Software Engineering Vol. 10, No. 9

Some, S.S. (2006), Supporting use case based requirements engineering, Information and Software Technology Vol. 48 No. 1pp. 43-58

Somerville, I. (2007), Software engineering, Addison Wesley

Spies, M. (2006), Vocabularies, Ontologies and Rule for the Enterprise (VORTE), IEEE enterprise distributed object conference

Stevens, P. and Pooley, R. (2005), Using UML: Software engineering with Objects and Components, Addison Wesley

Stevens, D.E. and Thevaranjan, A. (2009) A moral solution to the moral hazard problem, Accounting, Organizations and Society

Storey, N. (1996), Safety critical computer systems, Addison Wesley

Vraalsen, F., den Braber, F., Hogganvik, I., Soldal Lund, M., Stølen, K. (2004), The CORAS Tool-Supported Methodology for UML-Based Security Analysis, SINTEF ICT

Vraalsen, F. , Lund, M.S., Mahler, T., Parent, X., Stølen, K. (2005) Specifying Legal Risk Scenarios Using the CORAS Threat Modelling Language -Experience and the Way Forward. , Third International Conference on Trust Management (iTrust'05) 
Webb, K, and White, T. (2005), UML as a cell and biochemistry modelling language, Biosystems Vol. 80 Issue 3 pp. 283-302

Woodruff, J.M. (2005), Consequence and likelihood in risk estimation: A matter of balance in UK health and safety risk assessment practice, Safety Science Vol. 43 pp. 345-353

Wu, D. and Olson, D.L. (2008), Supply chain risk, simulation, and vendor selection, International Journal Production Economics Vol. 114 pp. 646655

Yin (1998), 'The abridged version of case study research', in Bickmen, L. and Rog, D.J. (ed.), Handbook of applied social research methods, pp 229-284. Sage Publications

Zachman, J.A. (1997), Enterprise Architecture: The Issue of the Century, Database Programming and Design,

Zwikael, O. and Sadeh, A. (2007), Planning effort as an effective risk management tool, Journal of Operations Management Vol. 25 pp. 755-767 


\section{A. - Restoration project study}

\section{Introduction}

This appendix provides an overview of the case study which investigated the relevance of the CORAS tool to risk management outside of the IT Security sector for which it was designed. In this case the tool has been applied to the Kings Norton restoration project.

\section{Restoration project business risk}

The restoration project case study focuses on the use of Strengths Weaknesses, Opportunities and Threats (SWOT) analysis as a basis for defining a business case to justify the sustainable re-development of heritage buildings.

The case study presents the workshop results and investigates the relationships between the areas identified within the workshops. The analysis uses mappings and weightings to priorities the strengths and opportunities for inclusion in the business case.

The CORAS methodology (Vraalsen et al 2004) for carrying out security analysis was also applied to this case study to analyse the ability to apply the assessment tool outside its intended domain. This analysis was carried out by Boiss (2005).

\section{$\mathrm{KN}$ restoration}

Kings Norton Parish is a diverse community with a range of incomes, living standards and backgrounds. The Old Grammar School, Saracen's Head, St Nicolas Church, its churchyard and The Green together form one of the largest complex of medieval buildings in Birmingham, and are therefore a matter of immense pride and tradition for the local community. However, funding and usage issues mean that the buildings are in need of repair, restoration, conservation and maintanance.

The Kings Norton Parochial Church Council is responsible for these three significant buildings. Together with the local community, the church created a vision for restoring these buildings and improving the provision and utilisation 
of these facilities. The Kings Norton Restoration Project is the tangible form of that vision and has grown out of many years of work by volunteers on the buildings.

The project is particularly concerned with the restoration and conservation of the Old Grammar School and the Saracen's Head. The Old Grammar School is a beautiful timber-framed structure that has sadly fallen into decay and is on English Heritage's "at risk" register. After continuing as a school for approximately two hundred years, the building fell into neglect at the beginning of the 19th century. Repairs were made in 1910 when a new external staircase was put in and again in 1951 after vandalism and further decay had taken their toll.

The nearby Saracen's Head was quite possibly the largest house of the royal manor during the 16th century. Currently being used as Parish offices and structurally intact, the house boasts highly decorative medieval workmanship, and the sophisticated building techniques confirm that the property held high status.

Through the Restoration Project, the community, Church, Sponsors and all well wishers hope to realise the dream that :

"The Grammar School would be restored and reopened as an educational facility for school parties. Classes on local history and how children were taught in the time of Thomas Hall would be held. In order for this scheme to work, the Saracen's Head will also need restoring, to provide facilities, disabled access, toilets, etc. The Saracen's Head will also function as a mixed-use community facility for Parish and secular activities, serving the people of Kings Norton."

The core aims and goals of the Project can be broken down into four key areas:

- Heritage: To conserve and restore all that is most valuable in the local 
heritage;

- Community: To develop full use for very varied church and community needs;

- Education \& Training: To develop full access and interpretation of the buildings and other local heritage; and

- Sustainability: Conserve and restore the rich local heritage and ensure that the buildings have a sustainable future.

In August 2004 Kings Norton's Saracen's Head together with the Old Grammar School won the second series of the BBC2 Restoration programme. In addition to the money raised by this programme the Heritage Lottery Fund (HLF) approved the restoration proposals for the HLF grant application Stage 1. The project team is currently working towards the Stage 2 grant application which it was hoped would be completed towards the end of 2005 but is still awaiting final approval.

The Restoration Project means a lot to the Church and the people living in and around Kings Norton; Hence the considerable time, money and human effort being invested in this project to produce effective results. This project not only reflects the economic aspirations of the community but is also closely woven into its emotional fabric. For a project of this scale which will have a significant economic and quality of life impact on the whole community, it is important to perform risk analysis and management, to prepare for and be aware of the weaknesses and vulnerabilities in this project. The risk analysis must be taken into acount in any decision making process related to the project. Where the level of risk is high mitigating control measures should be put in place.

\section{Restoration Project Context Identification}

At the heart and soul of this project is the need to restore and conserve historic buildings, at the same time finding full use for them. Various groups of people like the Church, Management Committee, Architects etc come together to arrive at a Business Plan for the project. The plan is based on various feasibility criteria and have to satisfy certain requirements along with 


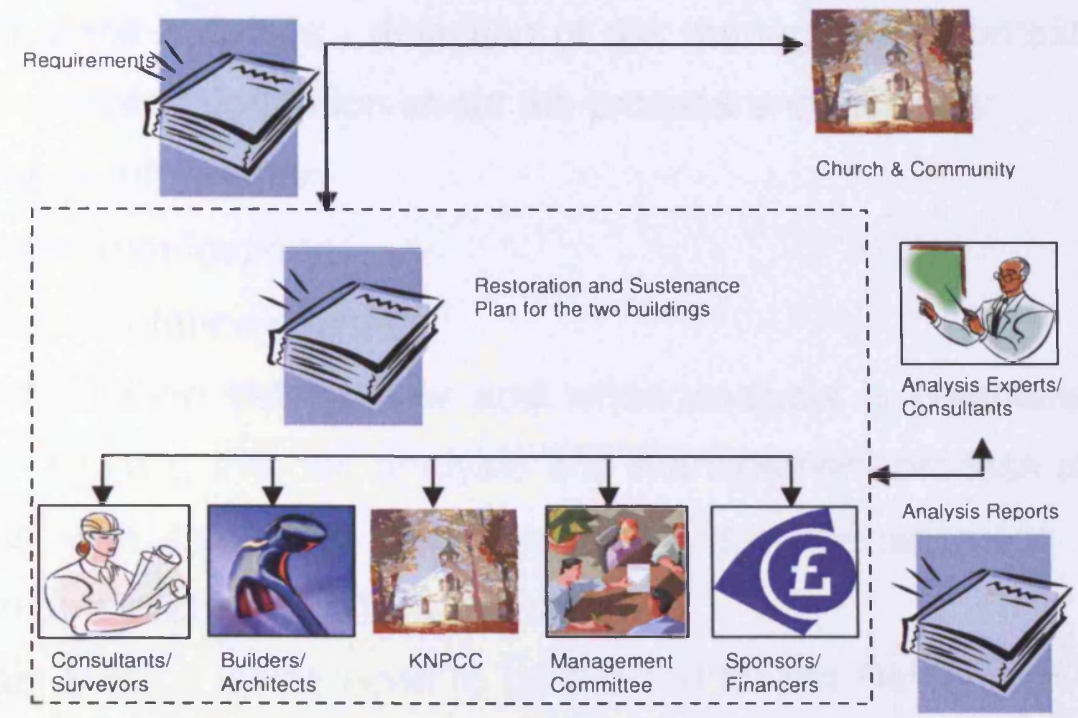

upholding the main aims of the project.

\section{Figure A-1 - Project view}

Figure A-1 uses UML models and text (Broendeland and Stolen 2004), the requirements are those related to the ultimate customers of this project, such as the Church and the local community.

Identify Context, as discussed in a paper on Risk Scenarios by Vraalsen et al (2005), involves the activities below:

- Definition of Risk Management Context;

- Evaluation of Target;

- Stakeholder Identification;

- SWOT analysis; and

- Asset Identification and valuation.

Throught these activities the following will be identified:

- The target owners for risk analysis;

- Purpose of this analysis;

- Assets;

- Scope of the analysis; and

- Risk acceptance criteria. 
The first of these activities - definition of risk management context - involves documenting meta information about the process and includes:

- Process Information;

- Domain metrics; and

- Risk acceptance criteria.

Process Information details how and when analysis is completed. For the Restoration Project, the risk analysis and management process is based on discussions with the local community and the management body. The information obtained is used for analysis.

The Domain Metrics which need to be defined for the Restoration project are as follows:

- Asset Values;

- Consequences Values;

- Frequency Values; and

- Risk Levels.

The final goal of the Restoration Project is the conservation and sustenance of the medieval buildings. Due to the nature of the project and the intangible nature of the assets, it is important to keep in mind that the values being defined here are qualitative in nature rather than quantitative, being based on historical and statistical data. Table A-1 shows the asset values identified for the project as a result of analysis based on workshops carried out with representatives of the people of the church.

\begin{tabular}{|l|l|}
\hline Asset Value & Description \\
\hline Very Low & $\begin{array}{l}\text { If it does not in any way hurt or reflect the } \\
\text { sentiments of the community. }\end{array}$ \\
\hline Low & $\begin{array}{l}\text { If it does not in any way hurt or reflect the } \\
\text { sentiments of the community. }\end{array}$ \\
\hline High & $\begin{array}{l}\text { If it is associated with the sentiments of the } \\
\text { community. }\end{array}$ \\
\hline Very High & $\begin{array}{l}\text { If it is associated with the sentiments of the } \\
\text { community and is important for the restoration of } \\
\text { the buildings. }\end{array}$ \\
\hline
\end{tabular}

Table A-1 Asset Values 
Time, Cost and Quality are three factors on which the Restoration project cannot compromise. Hence the consequence values, shown in Table A-2, are defined in terms of these three factors.

\begin{tabular}{|l|l|}
\hline $\begin{array}{l}\text { Consequence } \\
\text { Value }\end{array}$ & Description \\
\hline Insignificant & $\begin{array}{l}\text { If it does not in any way affect the cost, quality or length } \\
\text { of the project. }\end{array}$ \\
\hline Minor & $\begin{array}{l}\text { If it does not in any way result in the loss of time, cost } \\
\text { or quality. }\end{array}$ \\
\hline Moderate & If it involves more costs than estimated. \\
\hline Major & $\begin{array}{l}\text { If it delays the process and sabotages the chances of } \\
\text { grant approval. }\end{array}$ \\
\hline Catastrophic & $\begin{array}{l}\text { If it causes delays, increases the costs of service and } \\
\text { also reduces the quality of work. E.g. Failure of Stage 2 } \\
\text { Submission, Level of eligibility < 100\%, failure of the } \\
\text { grant application, etc. }\end{array}$ \\
\hline
\end{tabular}

Table A-2 Consequence Values

Frequency values are ranges defined by quanatative data described through examples or probabilities on a continuous scale. Table A-3 defines the frequency values for the Restoration Project.

\begin{tabular}{|l|l|}
\hline $\begin{array}{l}\text { Frequency } \\
\text { Value }\end{array}$ & Description( Probability) \\
\hline Rare & The probability of which is not likely at all. \\
\hline Unlikely & The probability of which is not very likely. \\
\hline Possible & $\begin{array}{l}\text { A situation which could happen within the application } \\
\text { period. }\end{array}$ \\
\hline Likely & Highly possible. \\
\hline Certain & Will happen within the application period. \\
\hline
\end{tabular}

Table A-3 Frequency Values 
At this stage, though the risks have not been identified yet, their levels need to be judged. These levels are identified in the form of a matrix as a function of Frequency and Consequence value to the Risk Level (Braber 2005). This matrix, shown in Table A-4, clearly gives the four Risks Levels will be applied during the course of this analysis.

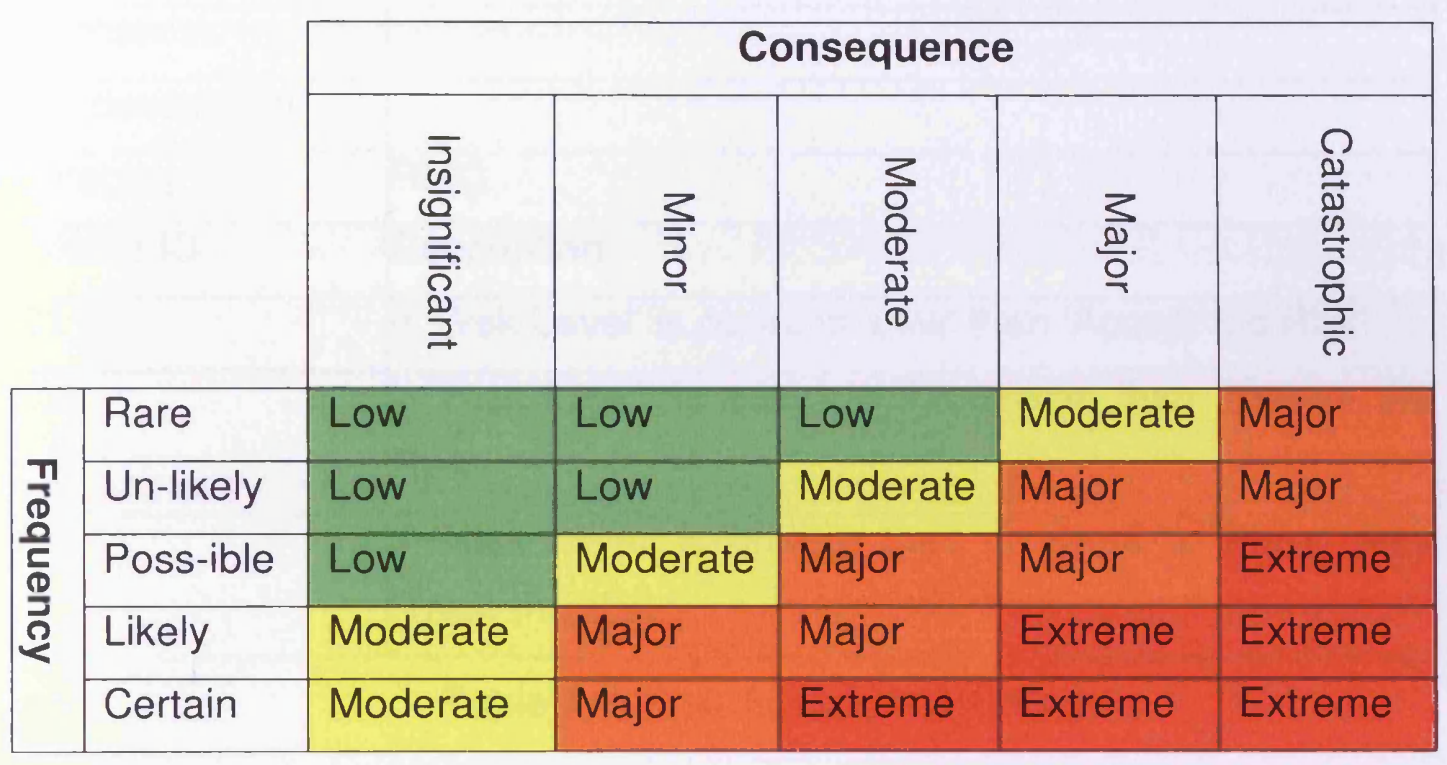

Table A-4 Risk Levels

Risk acceptance criteria. The Restoration project has qualified for the HLF Stage 1 and is currently working towards the completion of the application for the Stage 2 grant. One of the requirements for this Stage is that all permissions, including full planning permission and listed building consent have been obtained. The risk acceptance criteria for the Restoration project can be visualised using the CORAS tool and Table A-5 shows an export of that visulisation. 


\begin{tabular}{|l|l|}
\hline Type: & Table \\
\hline Name: & Risk Acceptance Criteria \\
\hline Short description: & Identifying the levels of acceptance \\
\hline Concern: & Risk management Context \\
\hline Viewpoint: & Engineering \\
\hline Finalised: & \\
\hline Full description: & \\
\hline Category & RMC \\
\hline Criteria ID & Description \\
\hline C1 & lf 'Risk Level' is equal to 'Low' then 'Accept the Risk' \\
\hline C2 & $\begin{array}{l}\text { If 'Risk Level' is equal to 'Moderate' then 'Monitor the } \\
\text { Risk' }\end{array}$ \\
\hline C3 & $\begin{array}{l}\text { If 'Risk Level' is greater than or equal to 'Major' then } \\
\text { 'Treat the Risk' }\end{array}$ \\
\hline
\end{tabular}

Table A-5 Risk Acceptance Criteria

The second of the activities - Evaluation of Target - details the focus of the Risk Assessment.

\begin{tabular}{|l|l|}
\hline Type: & Table \\
\hline Name: & Restoration Project-Target of Evaluation \\
\hline Short description: & Identifying the Target of this analysis \\
\hline Concern: & Target of evaluation \\
\hline Viewpoint: & Information \\
\hline Finalised: & \\
\hline Full description: & \\
\hline Category & ToE \\
\hline Target & $\begin{array}{l}\text { The Restoration Project in general and the two } \\
\text { buildings, The Old Grammar School and the Saracen's } \\
\text { Head, in particular }\end{array}$ \\
\hline Client & The Church and the Community \\
\hline
\end{tabular}




\begin{tabular}{|l|l|}
\hline Service/ Function & $\begin{array}{l}\text { Description of which parts of the system or organisation } \\
\text { in question are being analysed, including e.g. } \\
\text { references to diagrams or other relevant } \\
\text { documentation. }\end{array}$ \\
\hline Quality aspects & $\begin{array}{l}\text { Restoration and Sustenance of these buildings, their } \\
\text { availability for community use. }\end{array}$ \\
\hline
\end{tabular}

Table A-6 Evaluation of Target

Using the CORAS Tool the evaluation of the target can be expressed in a tabular form as shown in Table A-6. This clearly identifies and demarcates the Target, Client, Service and Quality aspects involved in the project.

The third activity - Stakeholder Identification - is used to identify groups with an interest in the project. Stakeholders can be catagorised into groups such as Customer, Sponsor and Supplier and are defined in Figure A-2.

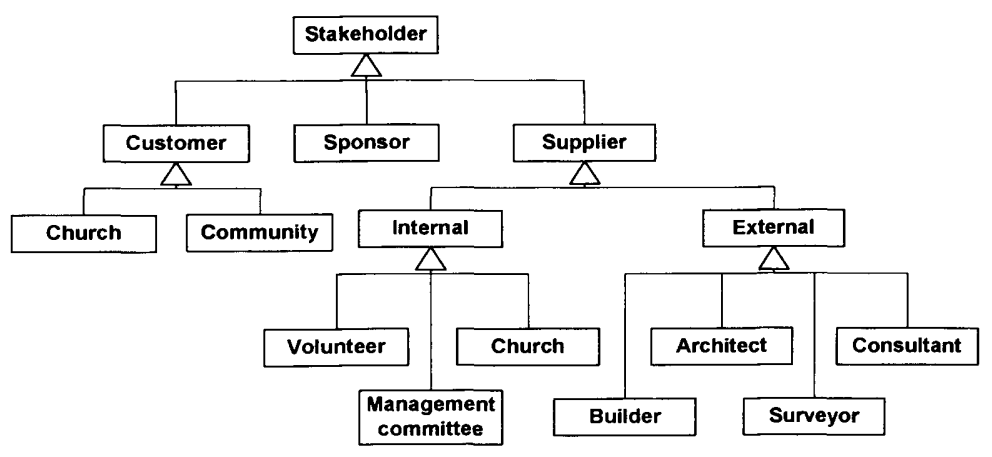

Figure A-2 - Stakeholders

The CORAS tool allows an expansion of this diagram using a table to give specific information about each stakeholder.

The fourth activity - SWOT Anaslysis - involves the understanding of Strengths, Weaknesses, Opportunities and Threats from Stakeholder perspectives. A workshop was conducted with Stakeholders in order to understand the current position and best direction for the Project. The objective was to identify the opportunities which can be successfully exploited and any strengths which will aid in their exploitation. 


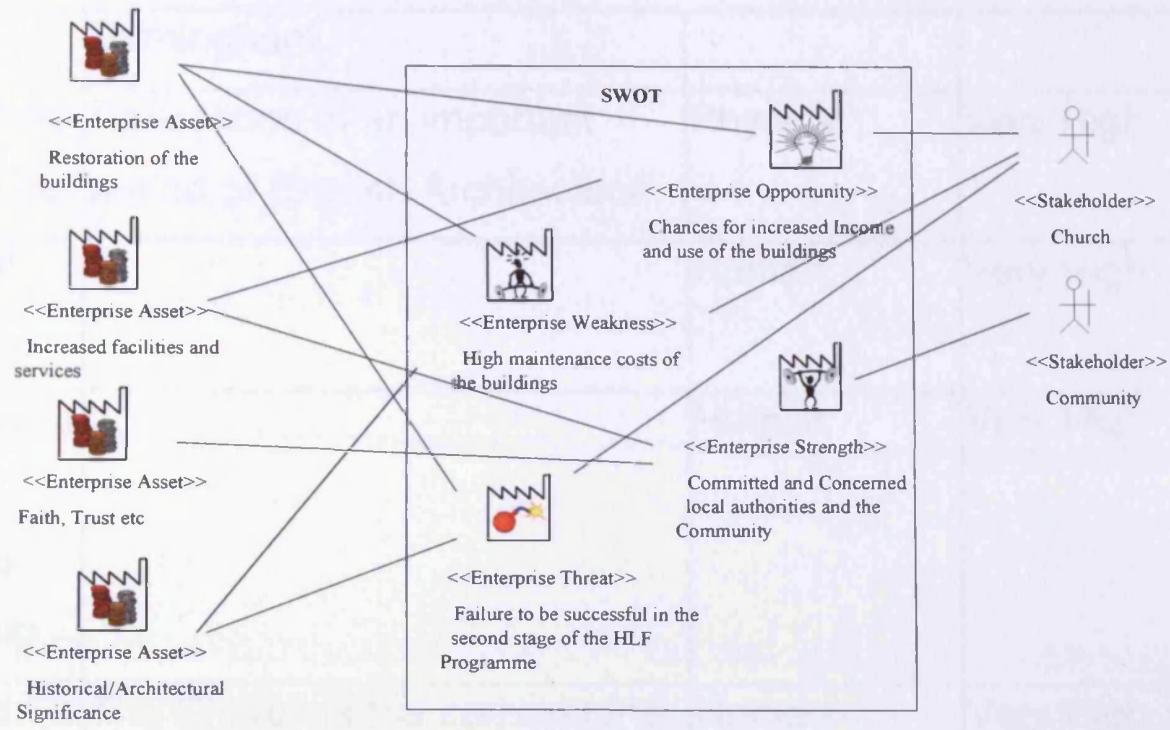

Figure A-3 - SWOT Analysis

The outcome of the SWOT workshop has been modelled using the CORAS Tool. Figure A-3 shows the stakeholders to the right of the diagram, their SWOT in the middle and Assets on the left. These Assets are the major areas of interest highlighted by the SWOT.

Asset Identification and valuation, the last of the activities, identifies the Target's features requiring protection. These so called Assests are the basis for the rest of the analysis (Braber 2006). The CORAS tool has been used to classify the various assets of the Restoration Project as shown below in Table A-7.

\begin{tabular}{|c|c|c|c|}
\hline Type: & \multicolumn{3}{|l|}{ Table } \\
\hline Name: & \multicolumn{3}{|l|}{ Identified Assets } \\
\hline Concern: & \multicolumn{3}{|l|}{ Assets } \\
\hline Viewpoint: & \multicolumn{3}{|l|}{ Organisational } \\
\hline $\begin{array}{l}\text { Full } \\
\text { description: }\end{array}$ & \multicolumn{3}{|c|}{ Identification and Valuation of the Assets } \\
\hline Asset ID & Description & Category & Value \\
\hline $\begin{array}{l}\text { Historical } \\
\text { Significance }\end{array}$ & $\begin{array}{l}\text { The buildings are part of one of } \\
\text { the largest complexes of } \\
\text { Medieval buildings in }\end{array}$ & Other & Very High \\
\hline
\end{tabular}




\begin{tabular}{|c|c|c|c|}
\hline & Birmingham & & \\
\hline $\begin{array}{l}\text { Architectural } \\
\text { Significance }\end{array}$ & $\begin{array}{l}\text { Reflections of an important } \\
\text { period of English Architecture. }\end{array}$ & Physical & Very High \\
\hline $\begin{array}{l}\text { Source of } \\
\text { solidarity }\end{array}$ & & Human & Very High \\
\hline $\begin{array}{l}\text { Commitment } \\
\text { and } \\
\text { Collective } \\
\text { Ownership }\end{array}$ & & Human & Very High \\
\hline $\begin{array}{l}\text { Faith and } \\
\text { Trust }\end{array}$ & $\begin{array}{l}\text { The Church is the cornerstone } \\
\text { of faith and trust for the } \\
\text { community. }\end{array}$ & Human & Very High \\
\hline Tradition & & Human & Very High \\
\hline $\begin{array}{l}\text { Sense of } \\
\text { Pride/Village } \\
\text { Identity }\end{array}$ & & Human & Very High \\
\hline $\begin{array}{l}\text { Education } \\
\text { and training }\end{array}$ & & Other & Very High \\
\hline Income & From guided tours to the sites & Physical & High \\
\hline
\end{tabular}

Table A-7 Identified Assets

It is important to note that the ultimate goal of the analysis is to understand the main concerns of the stakeholders in relation to the assets. The value of each asset is agreed by the stakeholders involved with the project.

Using the UML in accordance with the CORAS Methodology, assets that have been identified for the Restoration Project can be modelled. This model, shown in Figure A-4, provides structure and shows relationships between the various assets. 


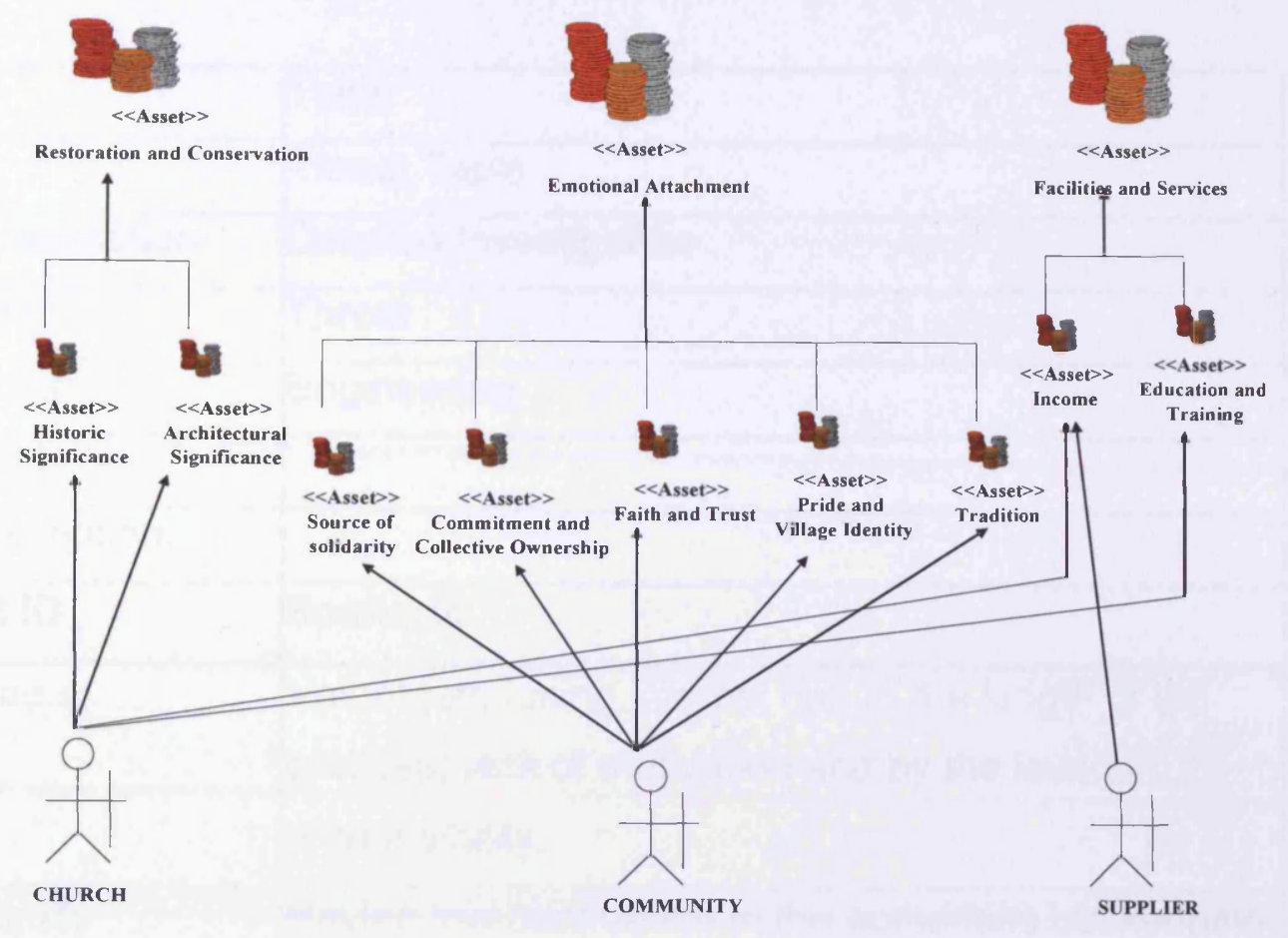

Figure A-4 - Asset Diagram

For the Restoration Project the stakeholders are the Church, the Community and the Suppliers. The assets can be categoriesed into three groups according to their nature. These are: Restoration and Conservation related to the Church Stakeholder; Emotional Attachment related to the Community Stakeholder; and Facilities and Services related to the Suppliers.

\section{Restoration Project Risk Identification}

Risk Identification in the CORAS methodology involves threat identification, identification of vulnerabilities in the system and identification of unwanted incidents. These three tasks involve structured brainstorming in the which relevant stakeholders will participate. Applying this to the Restoration Project, threats, see Table A-8, and vulnerabilities, see Table A-9, have been identified. Now identified these threats and vulnerabilities must be analysed to understand how threats may exploit the vulnerabilities resulting in Unwanted incidents and Threat scenarios, also detailed in Table A-8. 


\begin{tabular}{|c|c|}
\hline Type: & Table \\
\hline Name: & Threat Table \\
\hline Short description: & Detailed Investigation \\
\hline Concern: & Threat \\
\hline Viewpoint: & Engineering \\
\hline \multicolumn{2}{|l|}{ Finalised: } \\
\hline \multicolumn{2}{|l|}{ Full description: } \\
\hline Threat ID & Scenario \\
\hline Volunteers & $\begin{array}{l}\text { Volunteers losing interest due to the length of the } \\
\text { process, lack of motivation and by the level of } \\
\text { responsibility. }\end{array}$ \\
\hline Community & $\begin{array}{l}\text { Having low confidence in the authorities but keeping } \\
\text { unrealistic expectations of the project. }\end{array}$ \\
\hline Bad Weather & $\begin{array}{l}\text { Resulting in the delay in the surveys and analysis, } \\
\text { leading to inaccurate results. }\end{array}$ \\
\hline Faulty Equipments & $\begin{array}{l}\text { Resulting in the delay in the surveys and analysis, } \\
\text { leading to inaccurate results. }\end{array}$ \\
\hline Third Party & $\begin{array}{l}\text { Third Party consultants may be unavailable or may } \\
\text { not misunderstand the exigency of the tasks }\end{array}$ \\
\hline $\begin{array}{l}\text { Involvement of too } \\
\text { many people }\end{array}$ & $\begin{array}{l}\text { May lead to sense of competitiveness and non-co- } \\
\text { operation amongst the various groups of people, } \\
\text { spreading resentment and unhappiness. }\end{array}$ \\
\hline Criteria for eligibility & $\begin{array}{l}\text { Makes it appear a Herculean task, requiring huge } \\
\text { amounts of time, manpower (paid/unpaid) and other } \\
\text { resources. }\end{array}$ \\
\hline Commercial-isation & $\begin{array}{l}\text { The actual aim being compromised due to the } \\
\text { project becoming a commercial enterprise. }\end{array}$ \\
\hline $\begin{array}{l}\text { Stretched } \\
\text { Resources }\end{array}$ & $\begin{array}{l}\text { Due to the time required by the application process, } \\
\text { already-scanty resources being stretched. }\end{array}$ \\
\hline
\end{tabular}

Table A-8 Threat Table 


\begin{tabular}{|l|l|l|}
\hline Type: & Table \\
\hline Name: & Vulnerability Table \\
\hline $\begin{array}{l}\text { Short } \\
\text { description: }\end{array}$ & Detailed Investigation \\
\hline Concern: & Vulnerabilities \\
\hline Viewpoint: & Engineering & Asset \\
\hline Finalised: & & \\
\hline Full description: & & \\
\hline Vulnerability & \multicolumn{2}{|l|}{} \\
\hline Lack of resources & \\
\hline Poor condition of the buildings & \\
\hline Poor access facilities & \\
\hline Time Constraints & \\
\hline Unfavourable Ground Conditions & \\
\hline Accuracy and effectiveness of the surveys & \\
\hline Motivation & \\
\hline Understanding and Co-operation between the people \\
involved.
\end{tabular}

Table A-9 Vulnerability Table

The way in which threats can exploit vulnerabilities is highlighted by the use of the models and tables in the CORAS Tool and is depicted using threat scenarios. 


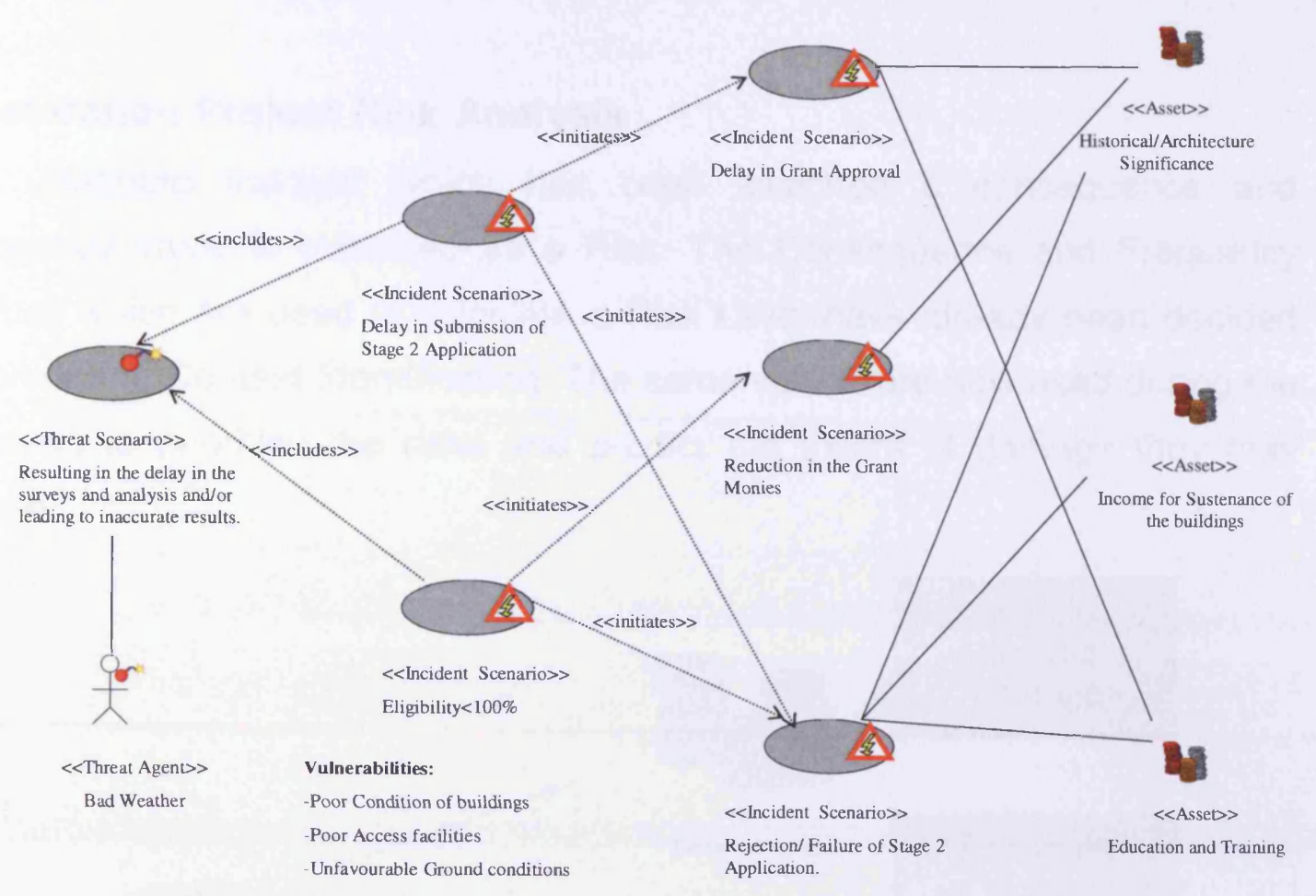

Figure A-5 - Threat Scenario 'Bad Weather'

Figure A-5 represents an unwanted incident model using the threat of Bad Weather. Bad Weather exploits the weaknesses existing in the system such as poor building conditions, poor access facilities to the buildings and unfavourable ground conditions. These vulnerabilities, when exploited by bad weather, aggravate the situation and this might result in the of delay in ongoing surveys or in the inaccuracy of the results. This in turn might delay the submission of the application or affect project eligibility causing rejection of the application or the reduction of the grant money.

During this stage in the project, it becomes imperative to have a clear idea of all possible dangers that the project may face and to plan countermeasures, so that the project aims are not compromised and the project does not suffer in terms of time, quality or costs. The modelling of unwanted incidents aids in this, and once a clear picture of all possible threats has been drawn, it becomes easier to prioritise these as risks and develop corrective treatment measures. 


\section{Restoration Project Risk Analysis}

An unwanted incident which has been assigned a consequence and frequency value is classified as a Risk. The Consequence and Frequency Values which are used to calculate a Risk Level have already been decided upon during Context Identification. The same values are now used during the analysis to prioritise the risks and predict the extent of damage they may cause.

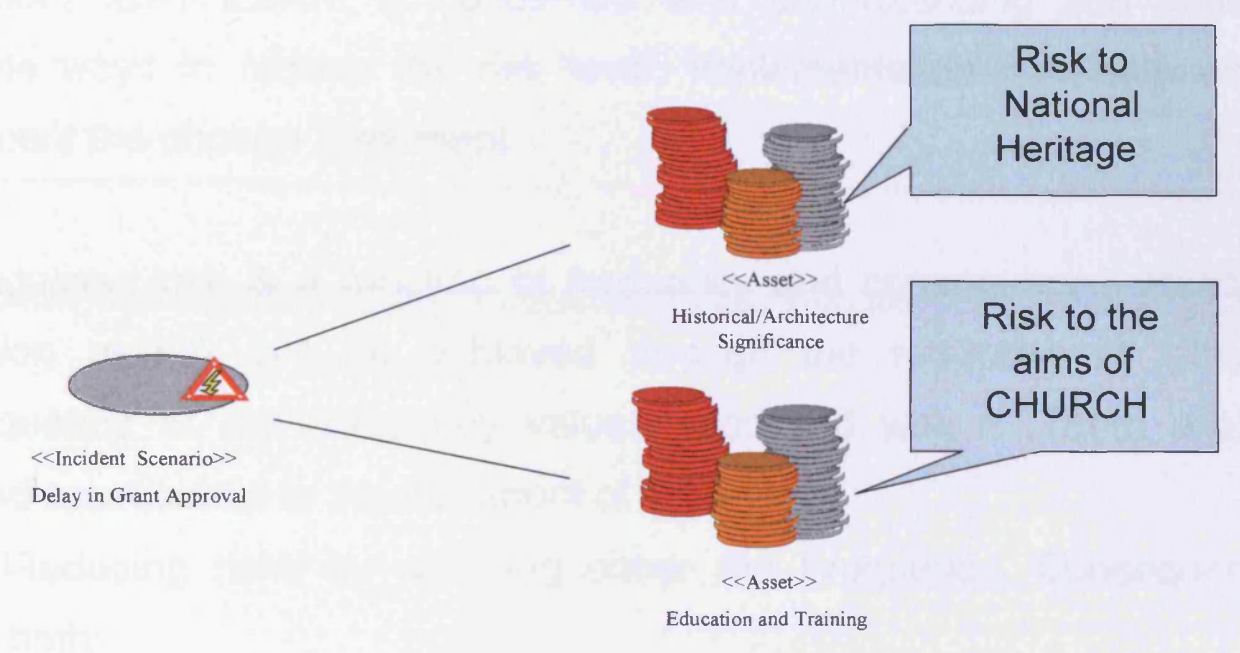

Figure A-6 - Risks and Assets

An unwanted incident may harm many assets. However, according to the CORAS Methodology, any risk is associated with only one asset. Depending on the stakeholder perspective and the assets being considered, the treatment measures can also vary. Due to the nature of the project it is wise to consider all the opinions and prioritise them according to their impact and level.

To understand the consequence and frequency of the risk we must take each Asset - Unwanted Incident pair. Taking the example of the 'Income' Asset and the 'Failure to generate enough money for the sustenance of the buildings' Unwanted Incident. This risk has been categorised as 'Major' from Table A-2, and attributed a frequency of 'Likely' from Table A-3. Overall this equates to the risk level of 'Extreme' based on the definitions in Table A-4.

It is expected that with this risk level the project will require further work to be 
completed in this area to fully understand what affects the risk and any variations to the unwanted incidents. It is also possible that this risk will be grouped with other risks which may, although affecting different assets, be caused by similar Unwanted Incidents.

\section{Restoration Project Risk Treatment}

Having identified and acknowledged the risks that require corrective measures, the next step is Treatment Identification and Implementation. Treatment Identification is concerned with understanding and evaluating possible ways to reduce the risk level; Implementation considers ways to implement the chosen Treatment.

As discussed risk is a function of frequency and consequence. Hence, the reduction of risk will be achieved through the reduction of either the consequence or the frequency value associated with it. There are three directed approaches to the treatment of risks:

- Reducing risks by reducing either the Frequency, Consequence or both;

- Transferring Risks e.g. By Insurance; or

- Not performing the Risky Activities.

The first two options are plausible and logical but the third, in the case of the Restoration Project, cannot be considered as it is imperative that all the surveys and studies, which have major risks associated with them, need to be successfully carried out.

The evaluation of these treatments involves taking each unwanted scenario and modelling the treatments in the same way. Consider the threat of 'Volunteers' which poses a possible threat of 'Losing interest in the process due to the duration of the application procedure and the level of responsibility.' If this unwanted scenario transforms into reality, it will directly or indirectly affect the assets identified for this project. Suitable corrective measures for this scenario could be:

- Regular meetings with the volunteers involved in the project which involves exchanging views and keeping everyone up-to-date with the status of the project; 
- The management being transparent with the volunteers; and

- Showing appreciation and encouragement.

Once the Control Measures have been identified, the feasibility (e.g. compatibility, user acceptance) and effectiveness (e.g. degree of prevention and level of risk mitigation) of the recommended corrective measure, discussed above, needs to be analysed.

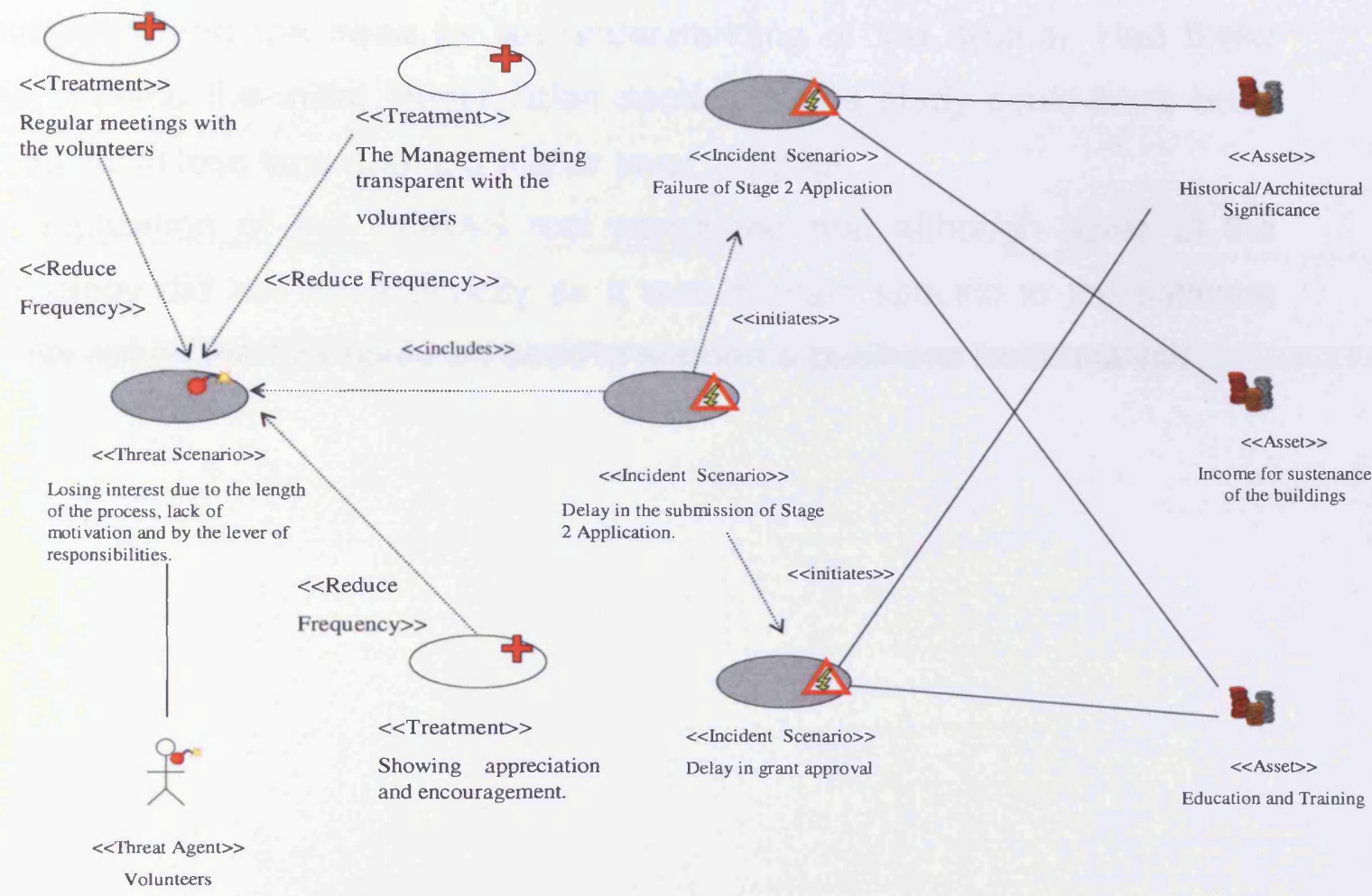

Figure A-7 - Treatment Model

The Treatments can be modelled to understand the threats that they help to reduce, as shown in Figure A-7. The risk can be re-analysed with each treatment, or combinations of treatment, allowing an understanding of the effect of the treatment on the overall risk level. Once a risk level has been reduced to an acceptable level a cost-benefit analysis is conducted to measure the effectiveness of this corrective measure or control strategy regarding the costs involved. The outcome of the cost benefit analysis may either suggest that treatments should be implemented, or that they would be prohibitively expensive and that other alternative treatments should be considered. 


\section{Conclusions - Business Risk}

The main conclusion from this study is that although many groups set out with good intentions many risk assessments are not clear on the route they should take to ensure that they are completed to a sufficient level of rigour to enable sound arguments to be built up to justify decisions.

The study supported the need to understand both the context of the assessment and the need for an understanding of the system. Had these been in place the initial identification section of the study could have been carried out in less time and to a higher level of rigour.

The application of the CORAS tool concluded that although some of the terminology did not trace directly as it was domain specific to the software security space the tool could be used to support a business level analysis. 


\section{B. - Modelling Language Overview}

\section{Introduction}

This Appendix provides an introduction to the constructs and usage of the Unified Modelling Language (UML) and Systems Modelling Language (SysML) diagrams. Initially it provides a brief overview of each of the thirteen UML diagram and nine SysML diagrams. Following each overview is a more detailed description of each of the diagrams used within this work.

The diagrams used within this work are:

UML

- Class

- Use Case

- Activity

- Sequence

- Deployment

SysML

- Parametric diagram

- Constraint block 


\section{UML Overview}

This section provides an overview of each of the six structural and seven behavioural diagrams

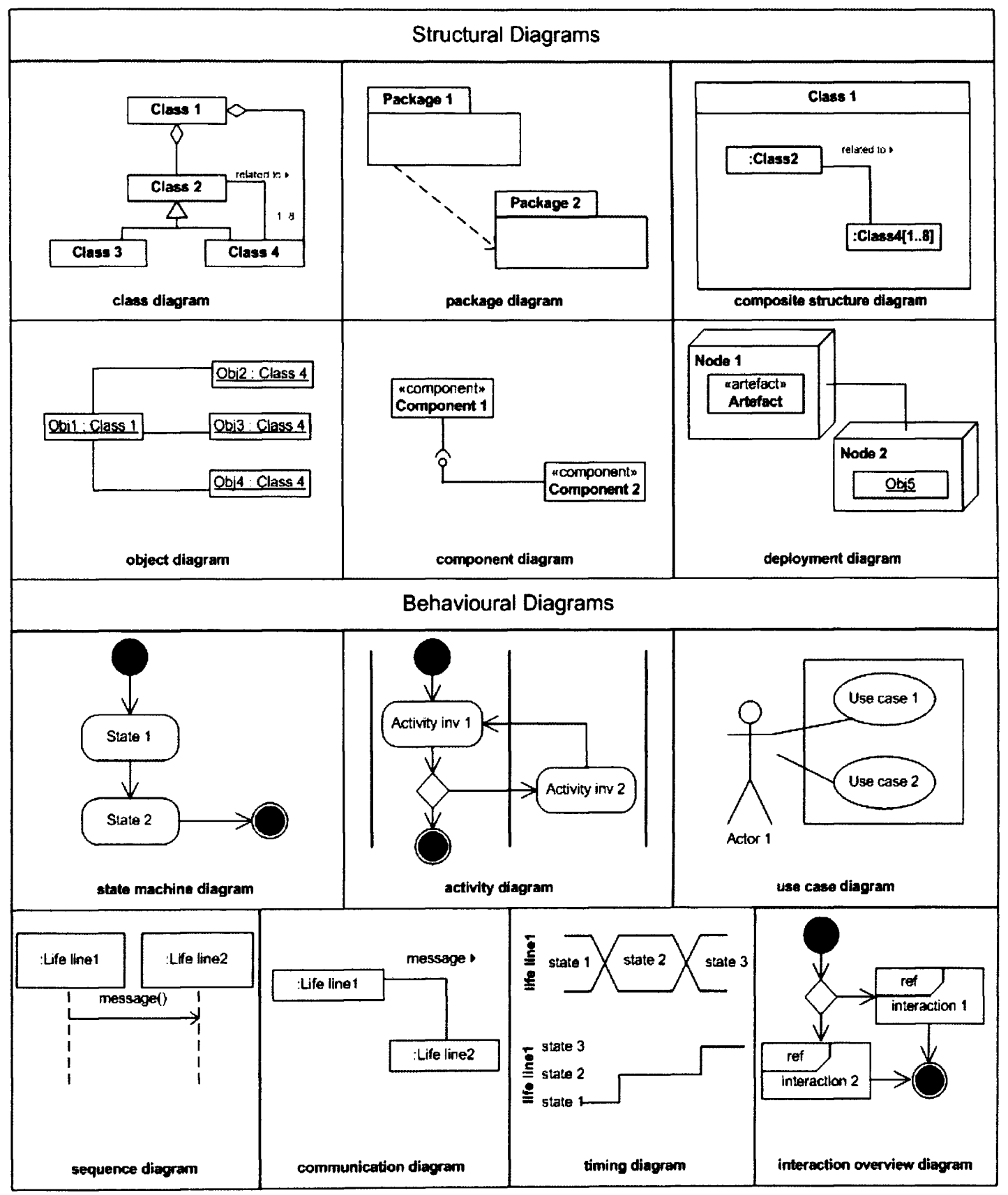

Figure B-1 - UML Diagrams Overview 


\section{Static Diagrams}

The class diagram provides the ability to show entities and their relationships. The relationships may be associations (including hierarchical), generalisations or dependencies.

The package diagram is generally used to show relationships within a model.

The composite structure diagram has two distinct purposes it realises compositions and aggregations, and collaborations which identify communications.

The object diagram shows instances of classes and relationships representing real life examples of the related classes.

The component diagram shows the modules that would be found within a system defined in terms of its interfaces.

The deployment diagram is used to define the location of components by placing them on nodes which represent real world aspects or locations.

\section{Behavioural Diagrams}

State machine diagrams show the behaviour of a object also described as the behaviour during the lifetime of a class. This is achieved by showing the order and conditions under which things occur.

Activity diagrams are special types of state machine which are generally used to show behaviour within an operation or state.

Use case diagrams are generally used to represent system requirements and contexts. They also show interactions with external systems or stakeholders.

Sequence diagrams show life lines, the timeline of an object, and the 
messages passed between them with an emphasis on the logical timing of the messages

Communication diagrams also show life lines and messages but the emphasis is on the layout or organisation rather than the timings.

Timing diagrams allows timing information to be added to interactions showing the time at which a message is sent or a state change occurs.

Interaction overview diagrams allow construction of complex behaviours by showing the interactions between many simpler behaviours described in scenarios.

\section{Class Diagram}

The class diagram provides the ability to show entities and their relationships. The relationships may be associations (including hierarchical), generalisations or dependencies.

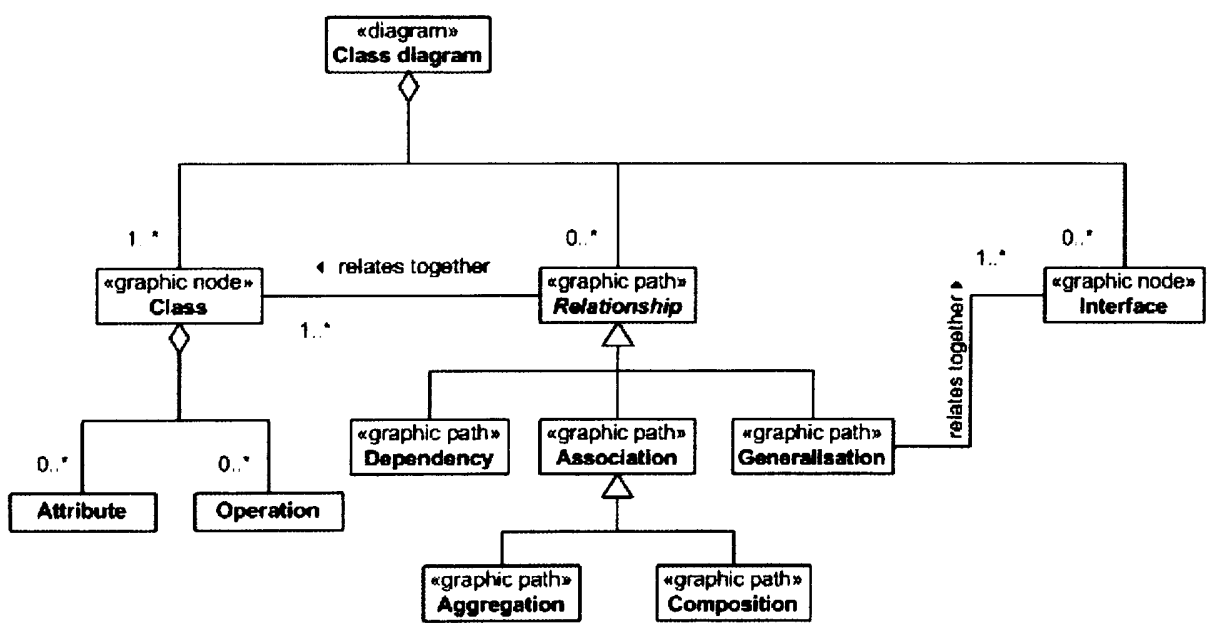

Figure B-2 - Class Diagram Meta Model

Figure B-2 shows the meta model (definition) of a class diagram, which is probably the most widely used diagram in the UML, it shows class and interface as graphic nodes; it also shows relationship and the three types described above. These relationships are dependency, association and 
generalisation. The association has two additional specialisations aggregation and composition. An example of the use of each relationships is shown below.

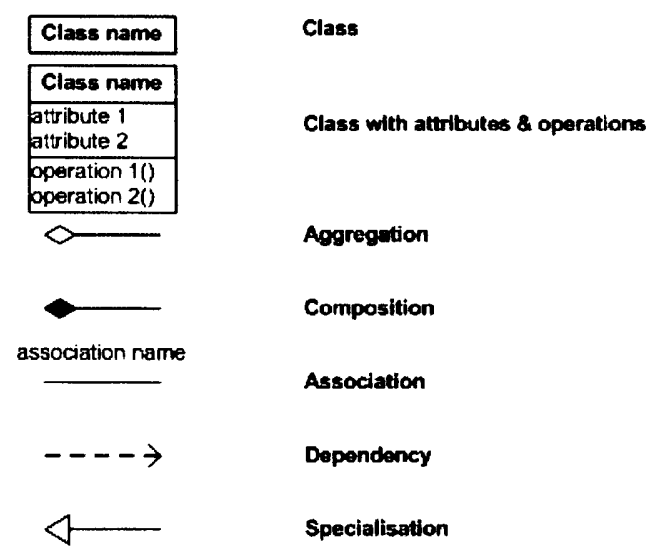

Figure B-3 - Class Diagram Symbols

The basic symbols to be used on the class diagram are shown in Figure B-3 the class can be seen to be a box which can have attribute and operation compartments added. The relationships are all in the form of lines; the dependency is a dotted line with an arrow to show the direction of the dependency, the generalisation/specialisation is a line with a triangle at the end of the parent class and the association is a plain line which may incorporate a name for the association. This line has a diamond added to the parent class end to show the two special types of association. 


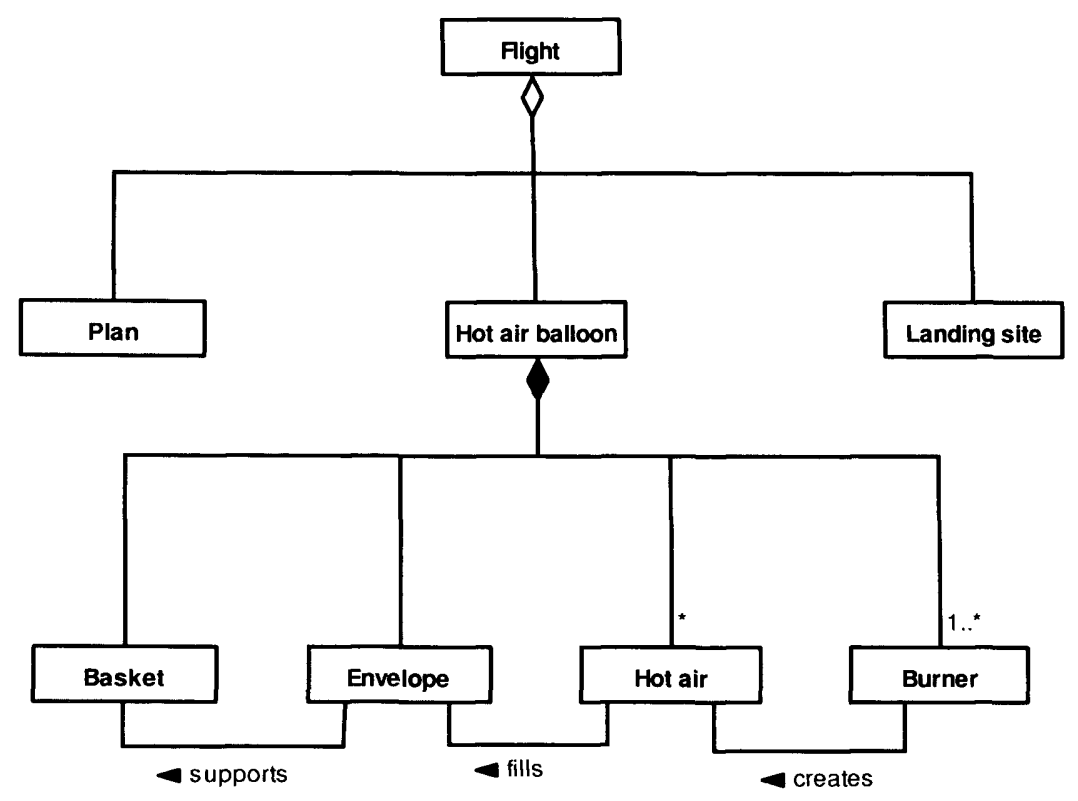

Figure B-4 - Example structure

The example structure in Figure B-4 shows all three associations; firstly a Flight is made up of one or more Hot air Balloon, a Flight Plan and a Landing site. Secondly the Hot air balloon is composed of one Basket, one Envelope, one or more Burner and many units of Hot air. The Basket is supported by one Envelope and one or more Burner which creates Hot air. The composition means that if you take away one of the component parts the whole does not exist i.e. if there is no Envelope there is no Hot air balloon.

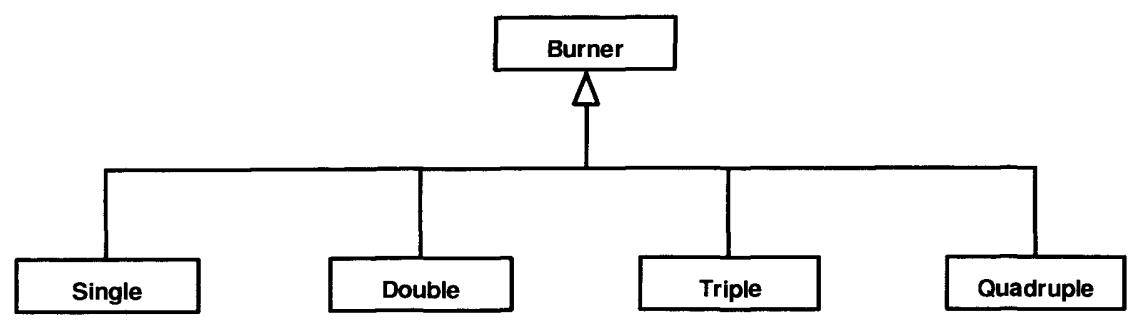

Figure B-5 - Example classification

Staying with the hot air balloon example there may be types of Burner. Figure B-5 shows single, double, triple and quadruple types of burner. 


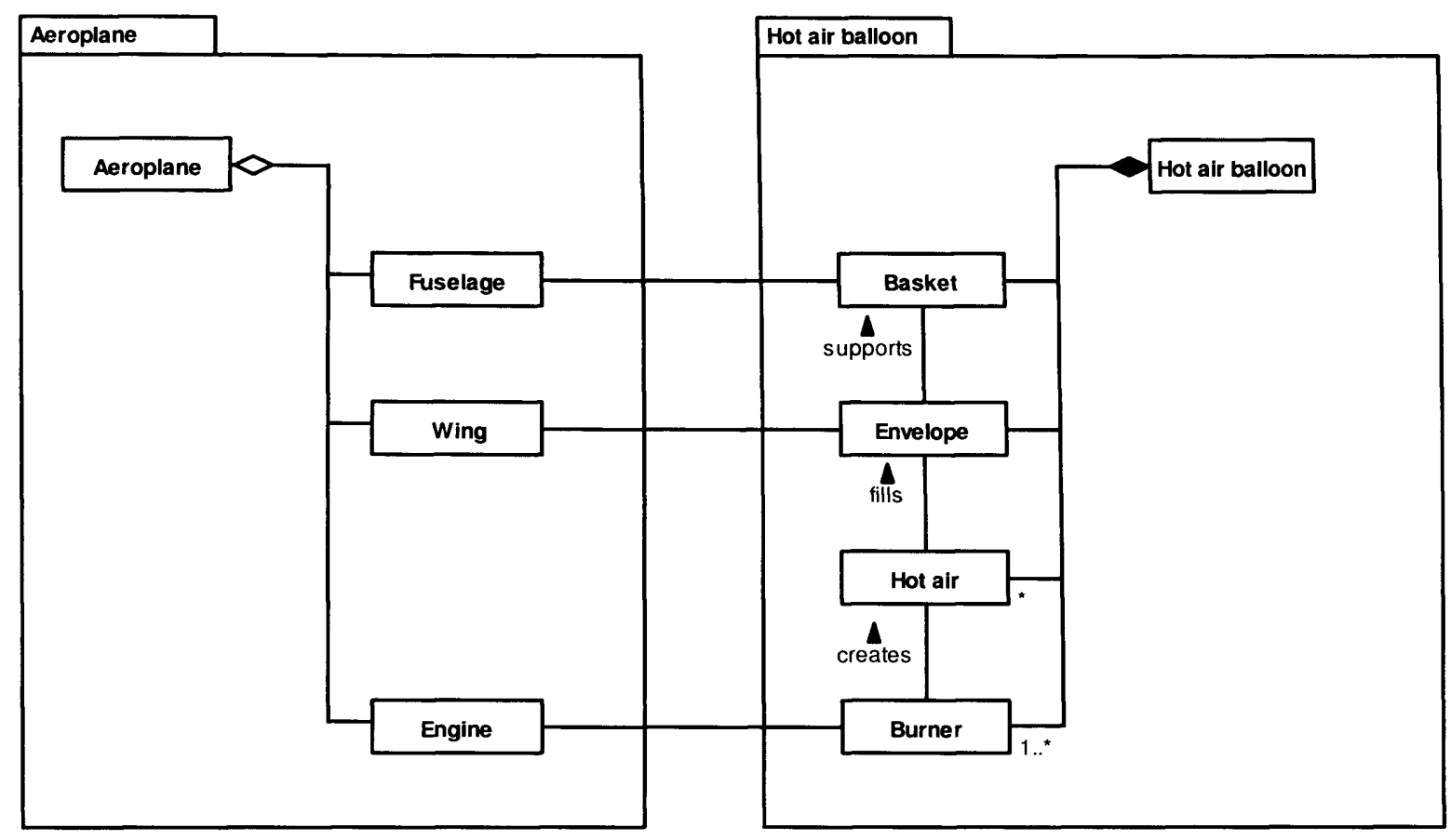

Figure B-6 - Example Mapping

Figure B-6 shows a mapping, an understanding of equivalence, using dependencies or associations this shows that the Basket of the Hot air balloon is maps to the Fuselage of an Aeroplane, the Envelope to the Wing and the Burner to the Engine. This can be very useful for showing equivalence between concepts used in different domains or applications. 


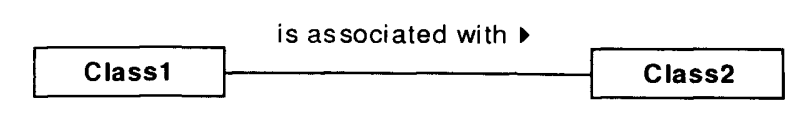

Class1 "is associated with" Class2

\begin{tabular}{|c|c|c|c|}
\hline & is as sociated with & & Class 1 "is associated with" "one or \\
\hline Class1 & & Class2 & \\
\hline
\end{tabular}

\begin{tabular}{|c|c|}
\hline Class1 & $\begin{array}{c}\text { Class1 "is made up } \\
\text { of" Class2 }\end{array}$ \\
\hline Class2 & $\begin{array}{c}\text { Class1 } \\
\text { composed of" } \\
\text { Class2 }\end{array}$ \\
\hline
\end{tabular}

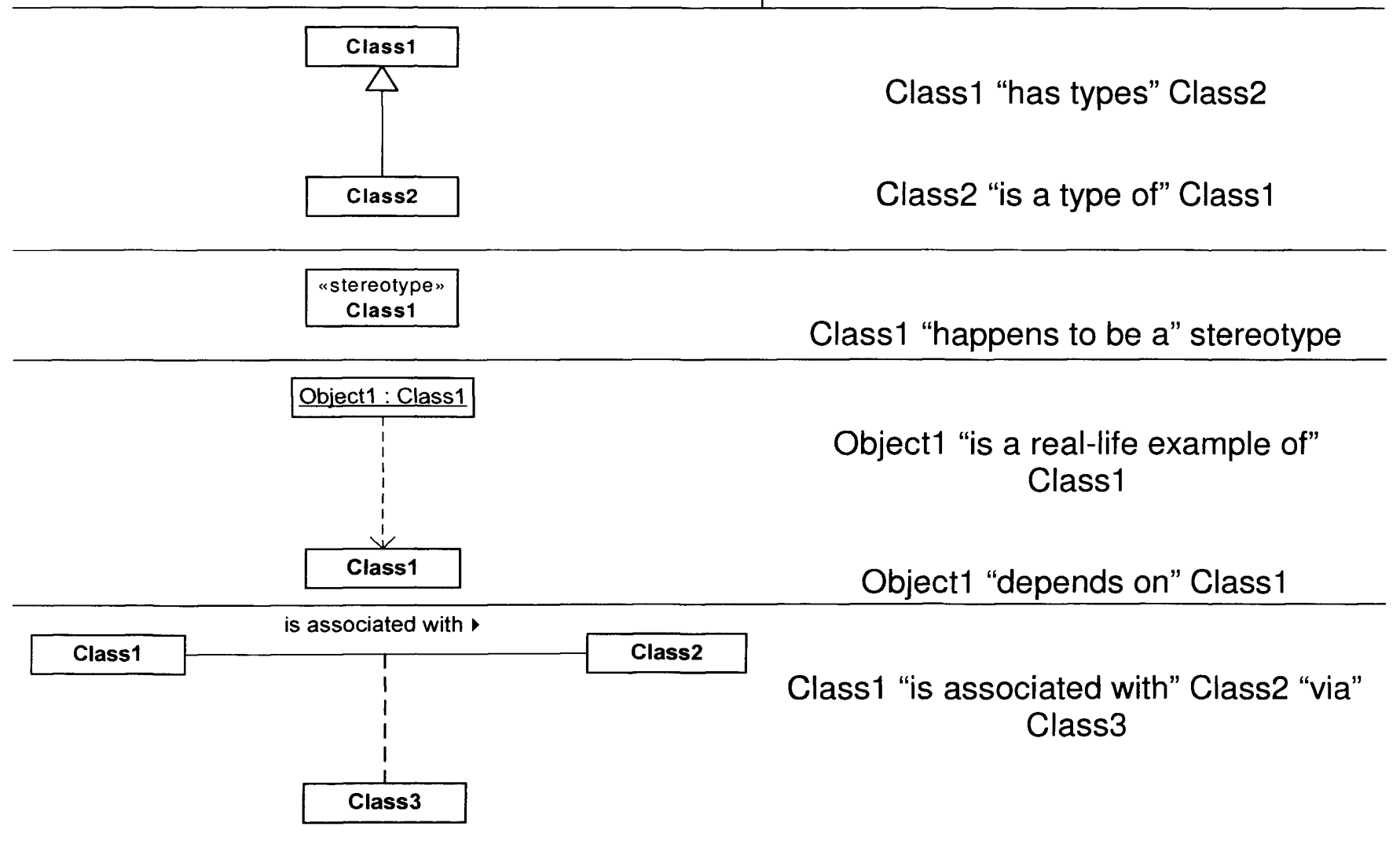

\section{Figure B-7 - Class Relationships Overview}

Figure B-7 provides a set of examples as an overview of the ways in which the relationships used on a class diagram can be read. 


\section{Activity}

Activity diagrams are low level diagrams and are generally used to show behaviour within an operation or state.

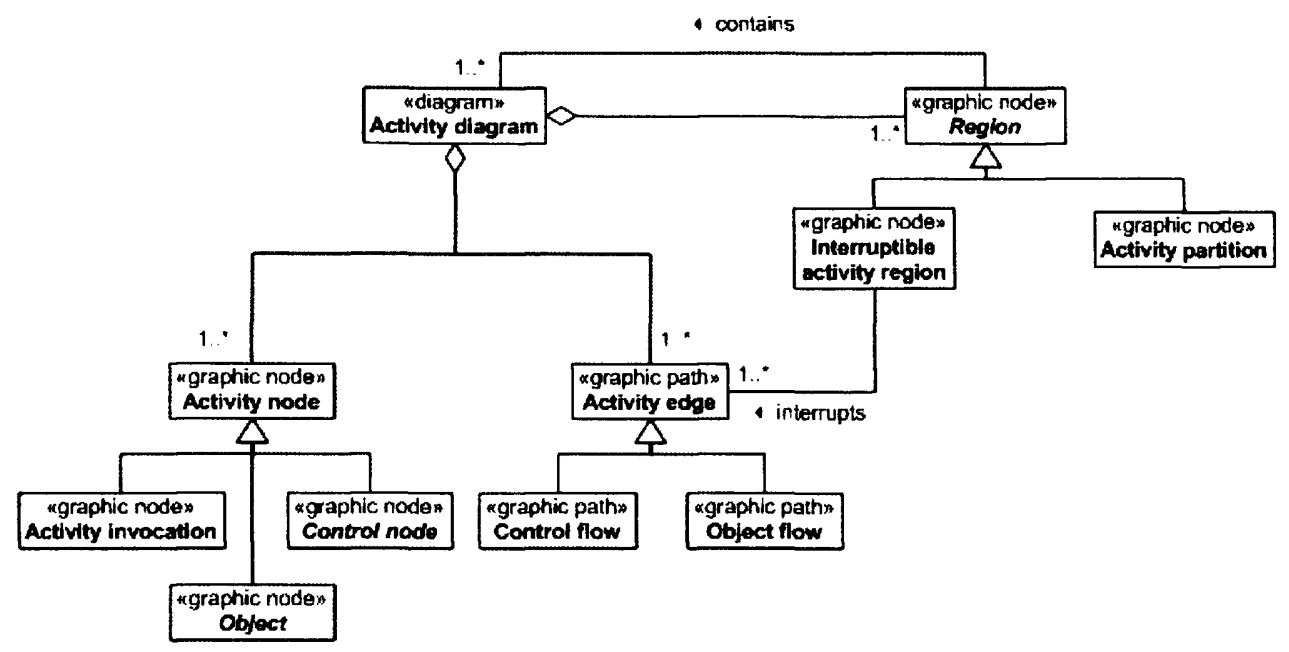

Figure B-8 - Activity Diagram Meta Model

Figure B-8 shows an extract of the meta model for the activity diagram. The diagram shows Activity nodes and Regions as graphic nodes, it also shows Activity edges these are the paths between the nodes.

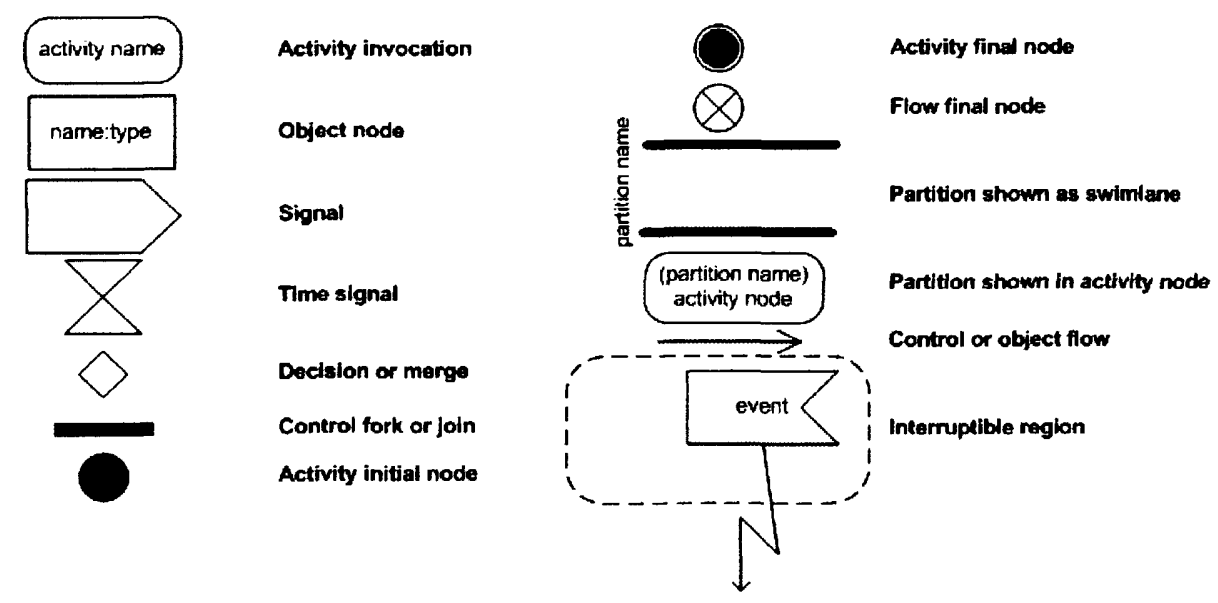

Figure B-9 - Activity Diagram Symbols

Figure B-9 shows the symbols used on the Activity diagrams. The activities 
are in the form of rounded boxes which are connected together via an arrow.

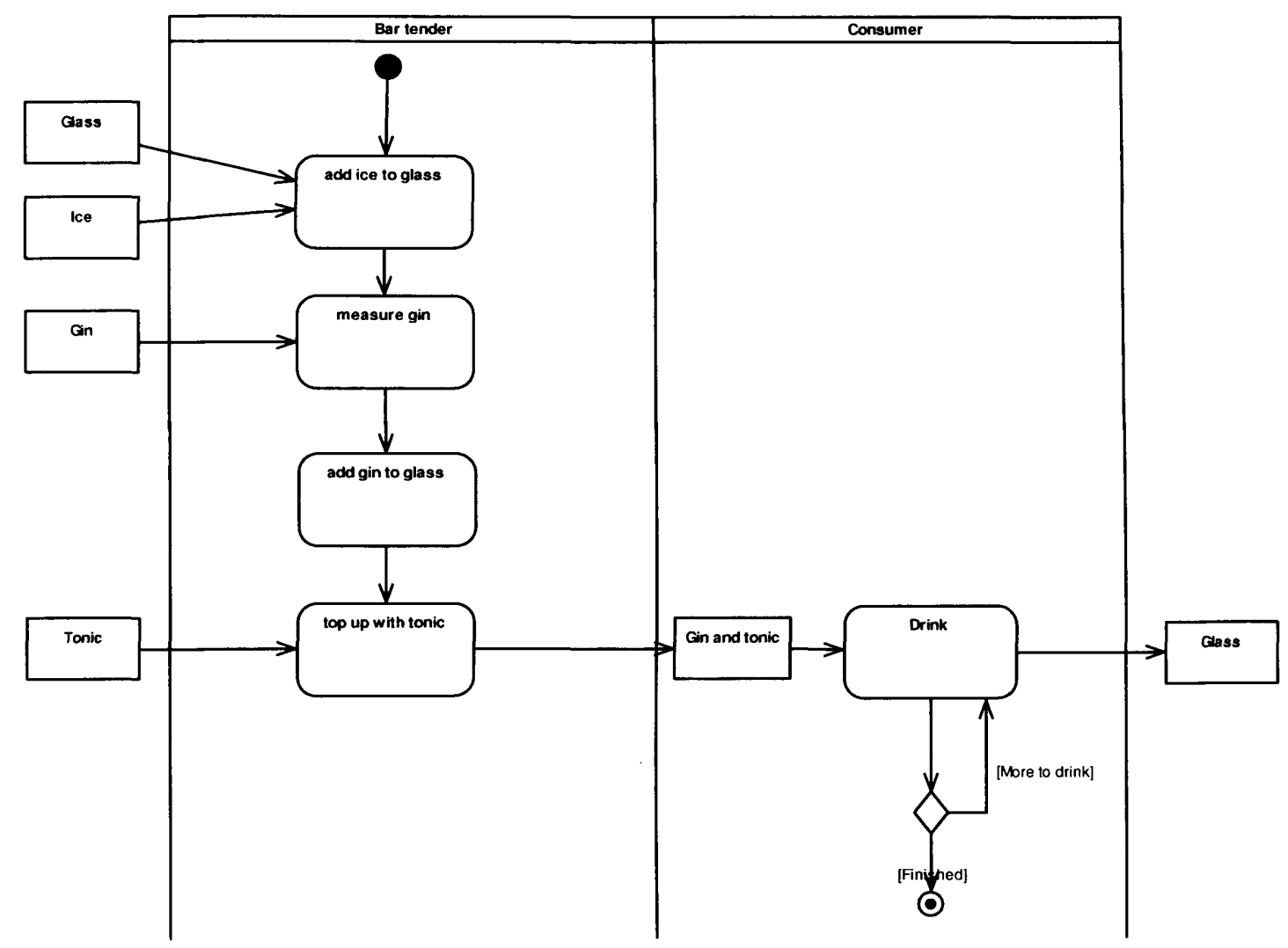

Figure B-10 - Example Activity diagram

Figure B-10 shows the activity diagram for pouring a drink. This shows the bar tender adding ice, adding a measure of gin and topping the glass up with tonic. At this point the bar tender passes the drink to the consumer who takes a drinks until they have finished enjoying each sip. 


\section{Deployment}

The deployment diagram is used to define the location of components by placing them on nodes which represent real world aspects or locations.

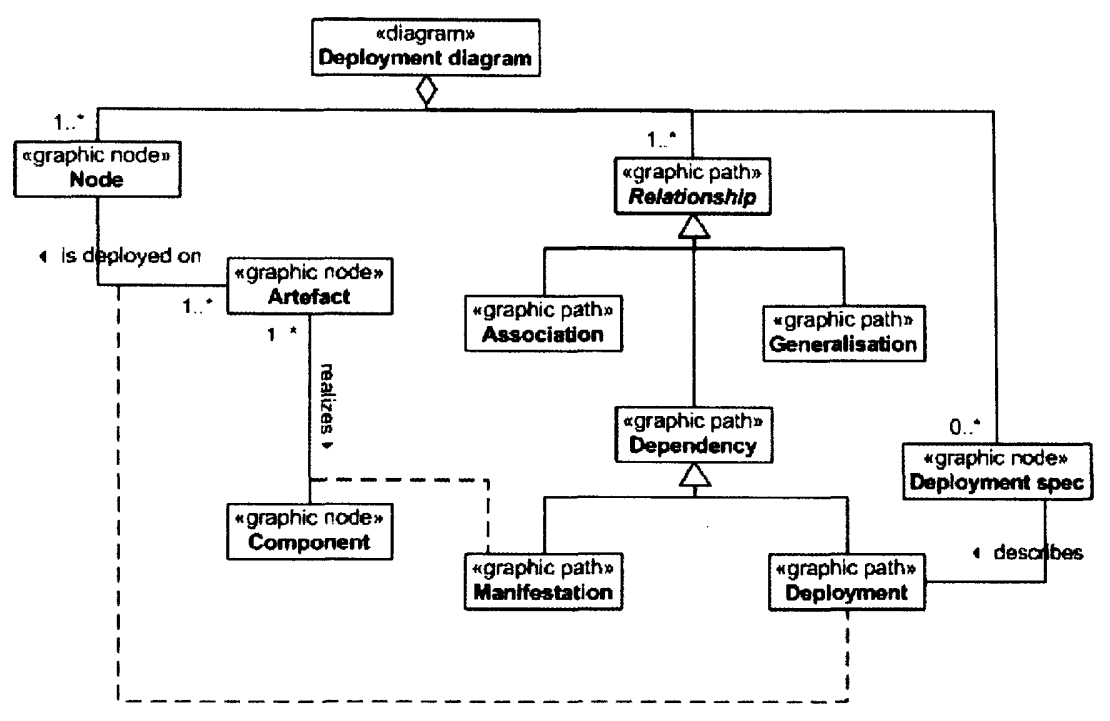

Figure B-11 - Deployment Diagram Meta Model

Figure B-11 shows an extraction of the meta model for a deployment diagram. It shows that Nodes, Artefacts and Components are depicted as graphic nodes whilst the relationships - similar to those in the class diagram - provide the paths between nodes.

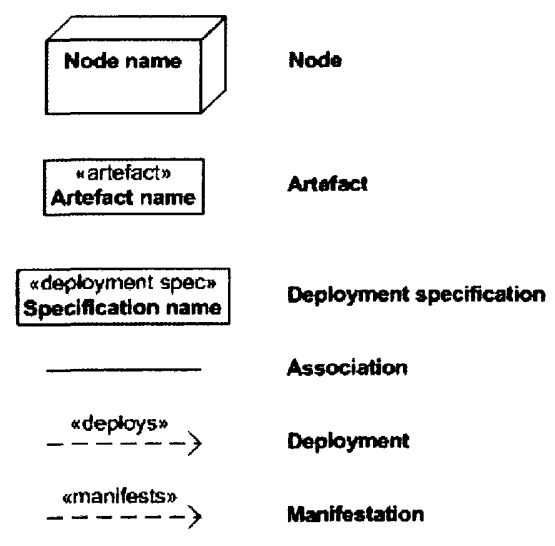

Figure B-12 - Deployment Diagram Symbols 
The symbols used on deployment diagrams can be seen in Figure B-12. The Node, the location, is shown as a 3D box onto which artefacts, components and deployment specifications can be placed. The association is similar to that used in the class diagram.

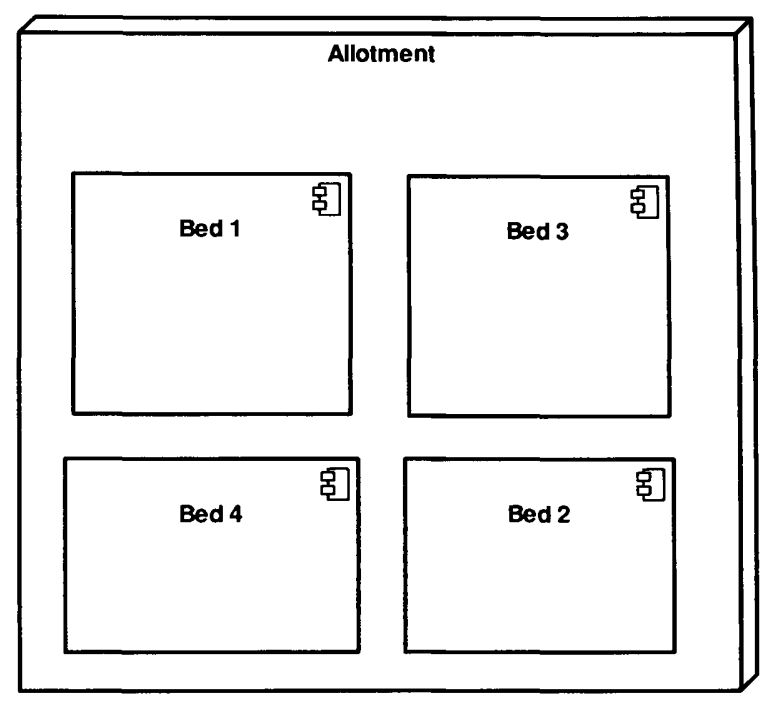

Figure B-13 - Example Deployment

Figure B-13 shows four planting beds in a crop rotation system. This shows where each of the beds is positioned within the allotment. It would is expected that deployment diagrams show either the current, past or future layout.

\section{Sequence}

Sequence diagrams show life lines, the timeline of an object, and the messages passed between them with an emphasis on the logical timing of the messages. 


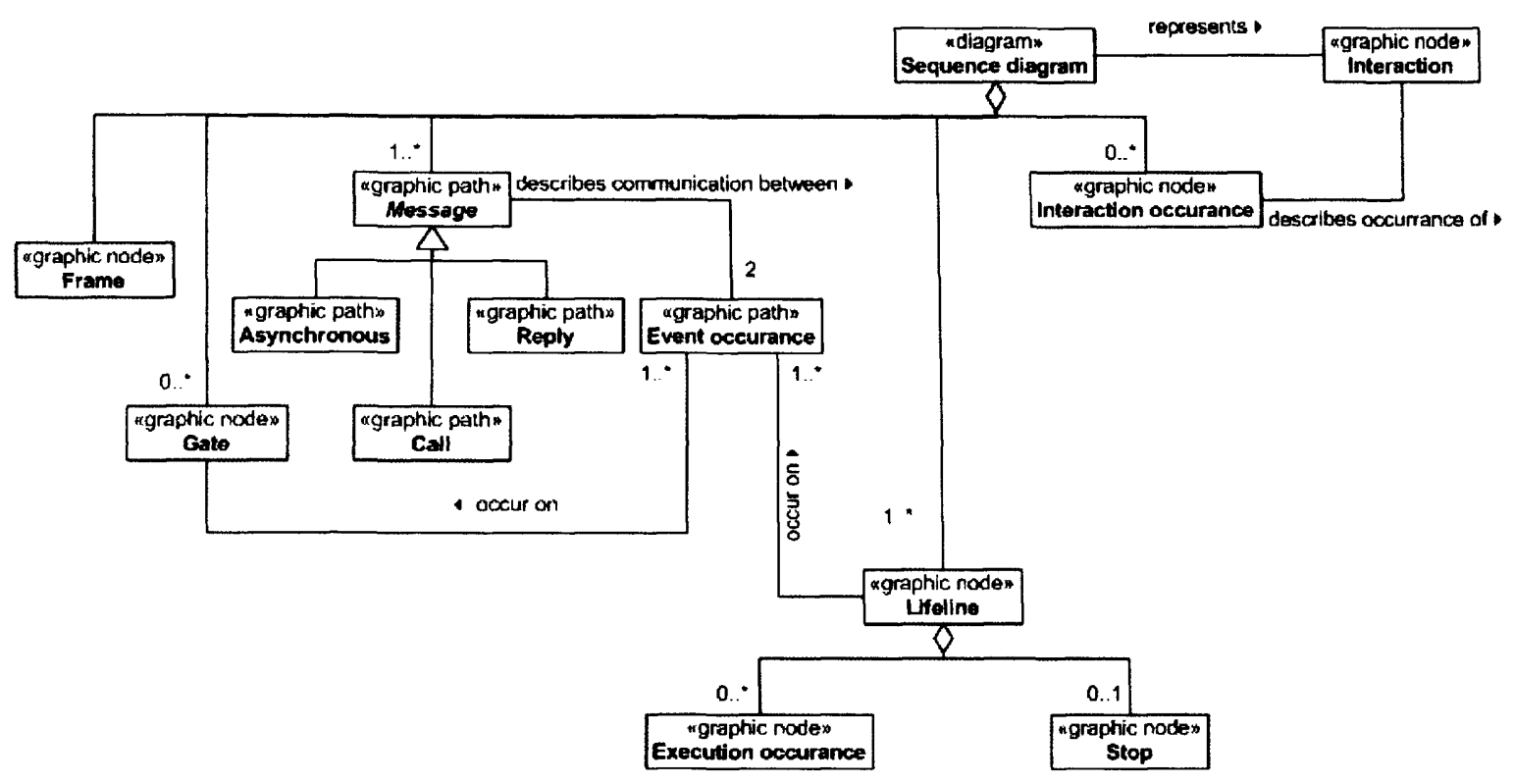

Figure B-14 - Sequence Diagram Meta Model

Figure B-14 shows an extract of the meta model for a sequence diagram. The diagram shows Frames, Gates, Lifelines, Interactions and Interaction occurrences occur as graphic nodes on the diagram. Messages provide the paths between events on lifelines.

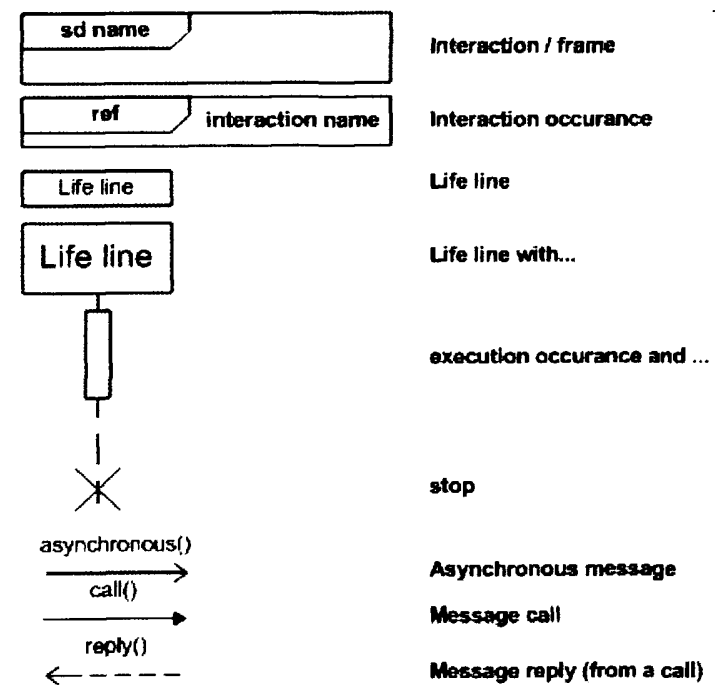

Figure B-15 - Sequence Diagram Symbols 
The symbols which can be used on sequence diagrams are shown in Figure B-15. This shows a lifeline as a box with a dotted line extending down the page. The messages between life lines are shown as arrows.

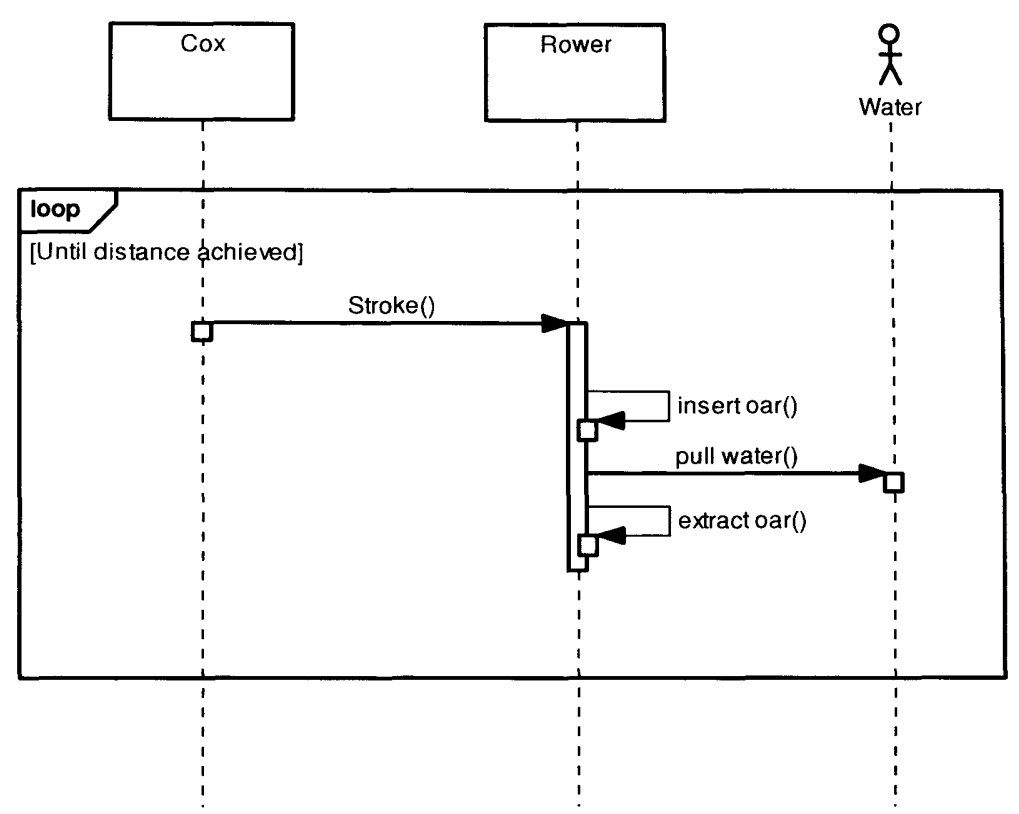

Figure B-16 - Example Sequence

Figure B-16 shows the sequence diagram for rowing. This shows the Cox calling the strokes to the rower at regular intervals with the rower reacting but inserting, pulling and removing the oar. 


\section{Use Case}

Use case diagrams are generally used to represent system requirements and contexts. They also show interactions with external systems or stakeholders.

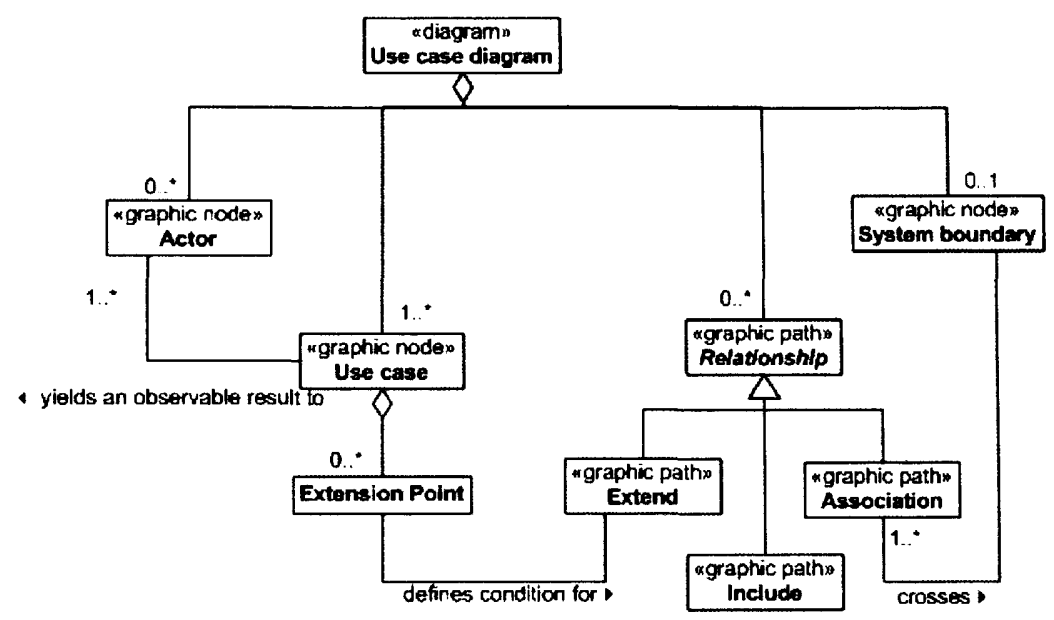

Figure B-17 - Use Case Diagram Meta Model

Figure B-17 shows an extract from the meta model of a Use case diagram. The diagram shows that Actors, Use cases and system boundaries can be shown on diagrams as graphic nodes and relationships used to provide paths between and across nodes. An association relates a Use case to an Actor usually across a system boundary. The Include relationship shows a Use case which always occurs when the source use case occurs. 


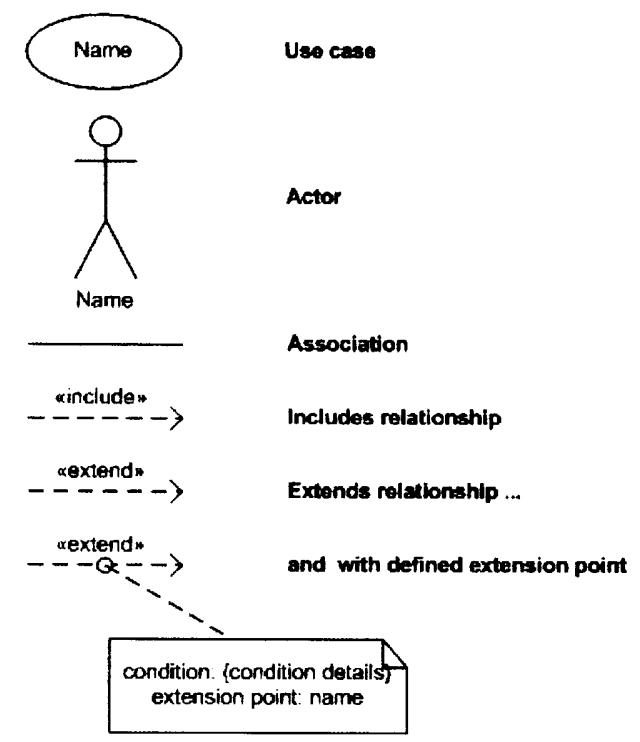

\section{Figure B-18 - Use Case Diagram Symbols}

The symbols which may be used within a Use case diagram are shown in Figure B-18. The diagram shows a use case as an ellipse and an actor as a stick man. The relationships are shown using the dependency arrow with the relationship type in chevrons e.g. <<include>>.

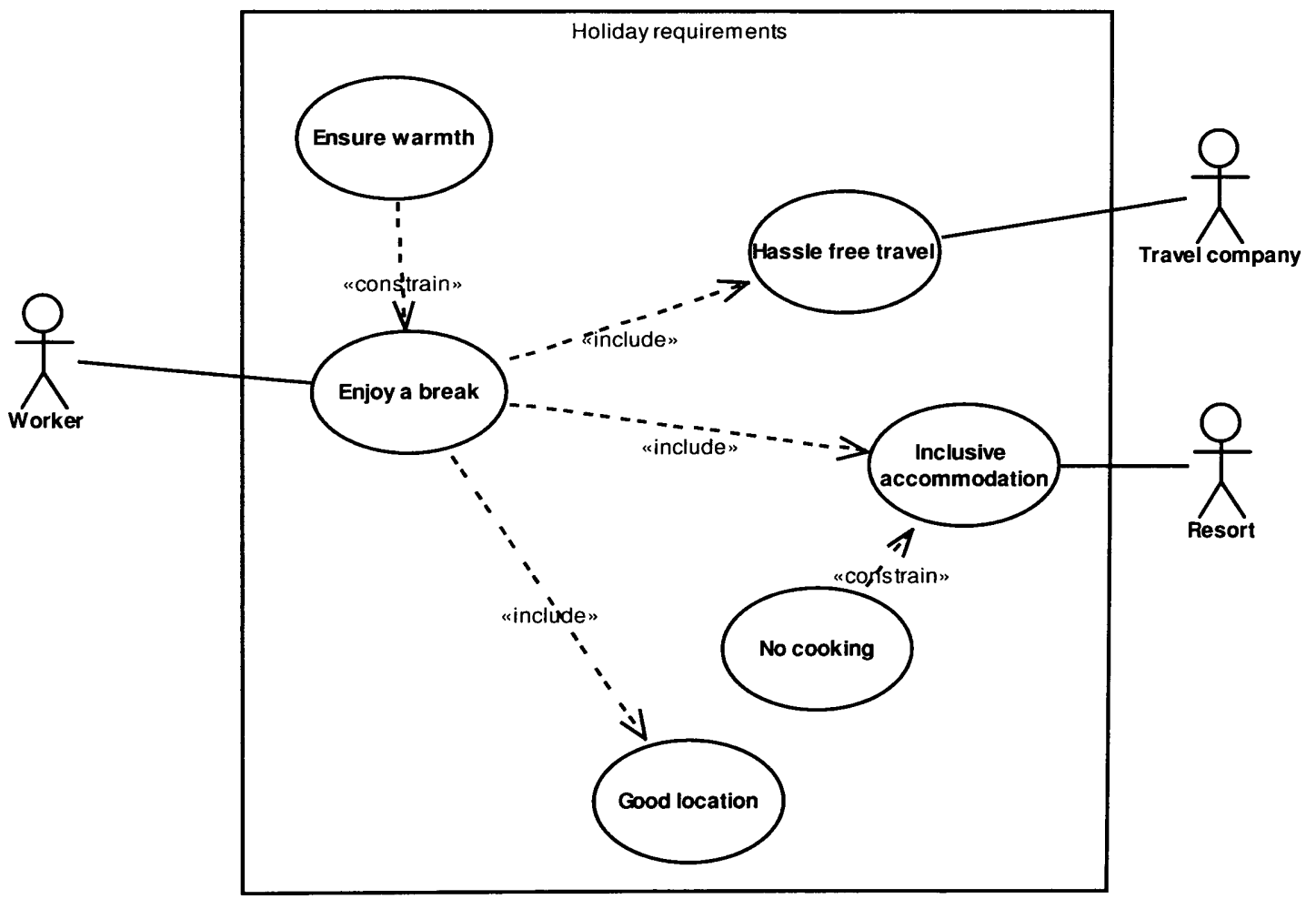

Figure B-19 - Example Use Case 
The use case diagram in Figure B-19 shows the requirements for a summer holiday showing the main requirement is to which the Worker is interested in is Enjoy a break, to do this the worker wants Hassle free travel which a Travel company will be interested in selling, Inclusive accommodation and a Good location provided by the Resort. An extra relationship, constrain, has been defined to be used on the use case diagram. This relationship can be considered to relate non-functional requirements.

The main constraint on the holiday is that it must Be warm whilst a lower level constraint on the accommodation is that there should be No cooking for the Worker.

\section{Diagram Relationships}

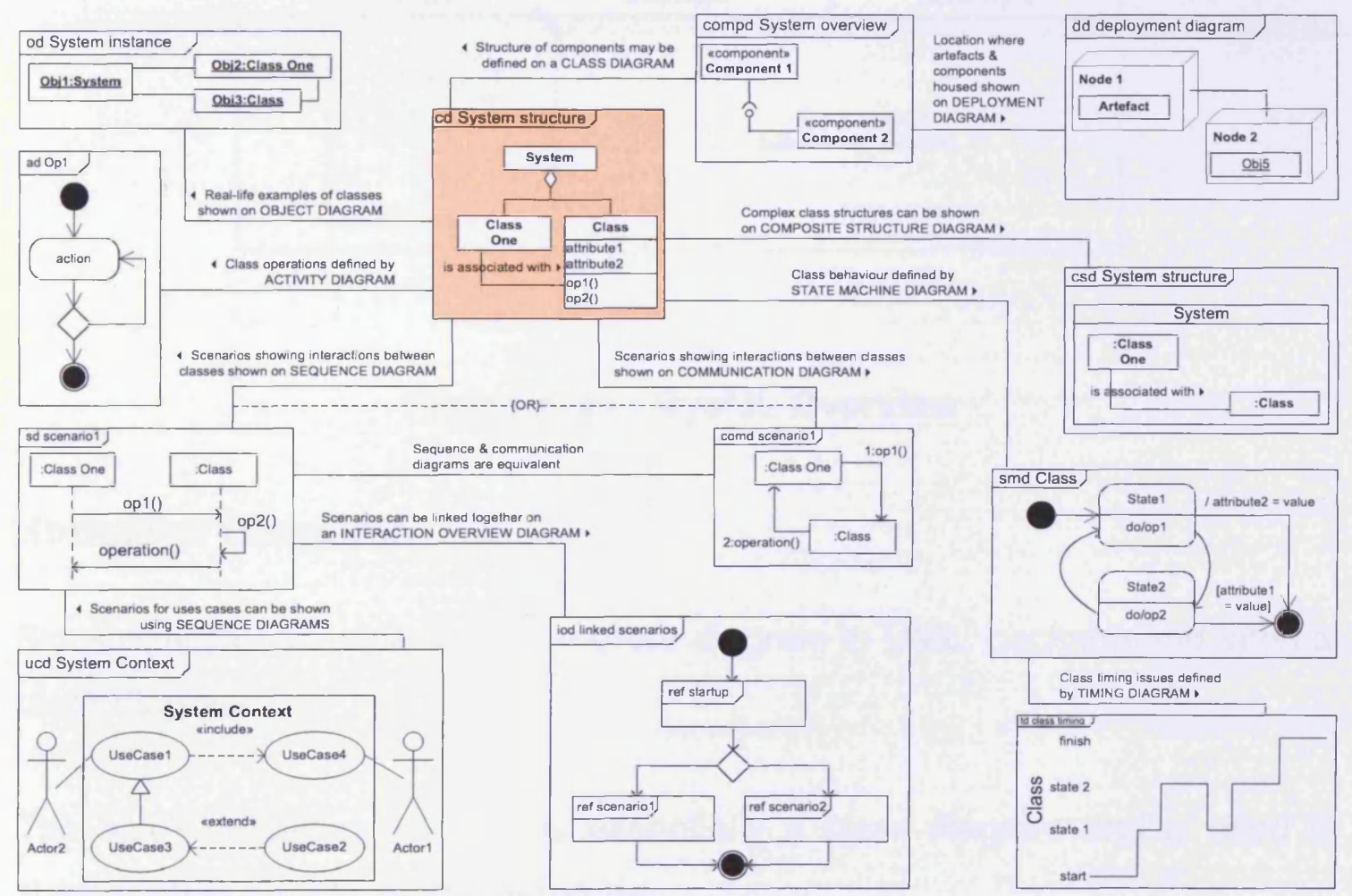

Figure B-20 - UML Diagram Relationships

Figure B-20 shows the relationships between each of the UML diagram, it is these relationships which support the consistency which the language provides as it enables the consideration of different views on the system being considered. 


\section{SysML Overview}
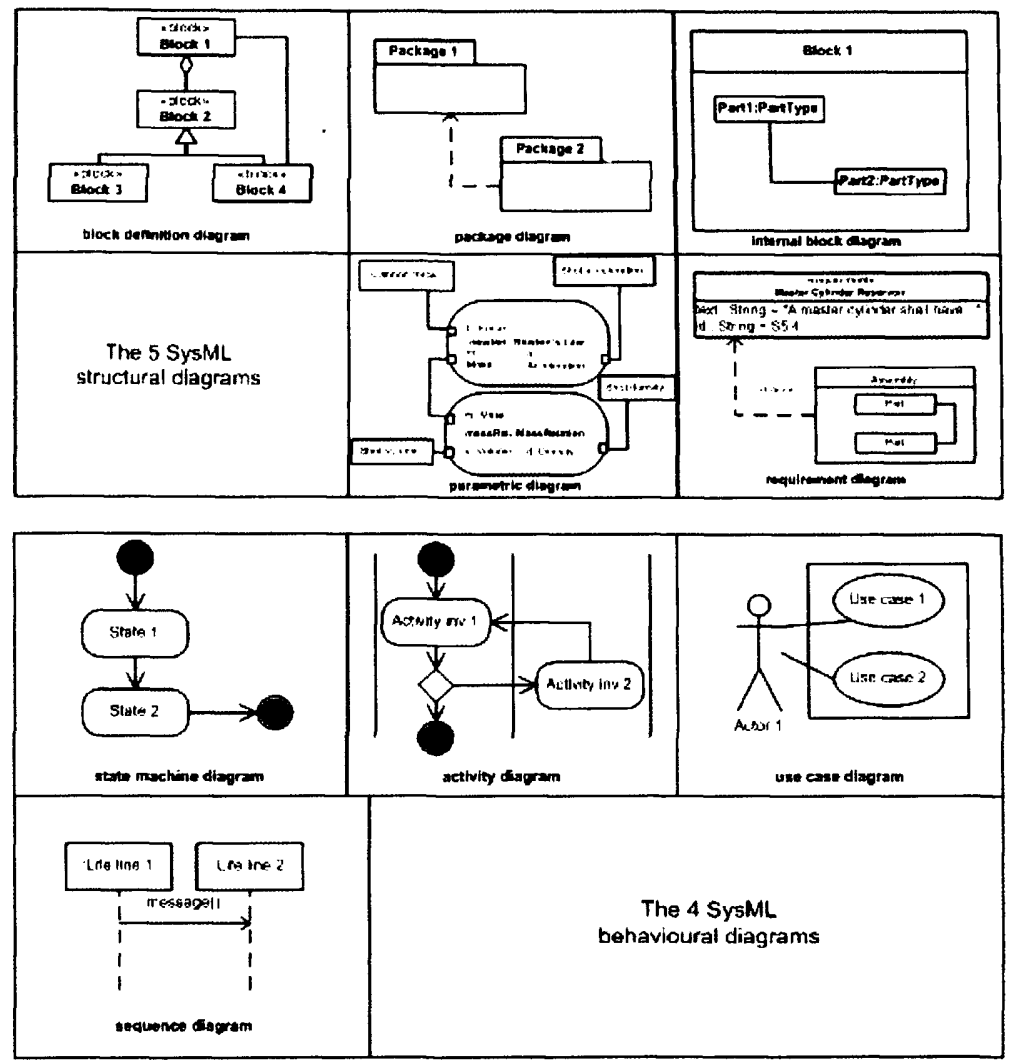

Figure B-21 - SysML Overview

\section{Structural Diagrams}

Block definition is essentially the class diagram in UML, package and internal block diagram are

The block definition diagram is essentially a class diagram and is used to show system structure and hierarchy.

The package diagram is used in the same way as the package diagram in the UML

The internal block diagram is essentially the composite structure diagram from the UML 
The parametric diagram provides the ability to show mathematical constraints and organise them into networks which can be exercised providing numerical and logical results.

The requirement diagram provides the ability to represent a requirement as a block or class.

\section{Behavioural Diagrams}

All of the behavioural diagrams in the SysML are commensurate with the diagrams of the same name in the UML.

\section{Parametrics}

The Systems Modelling Language (SysML) has introduced a new construct to the modelling toolbox, that of the constraint block and the associated 'parametric diagram'. Constraint blocks allow for the definition and use of networks of constraints that represent rules that constrain the properties of a system or that define rules that the system must conform to.

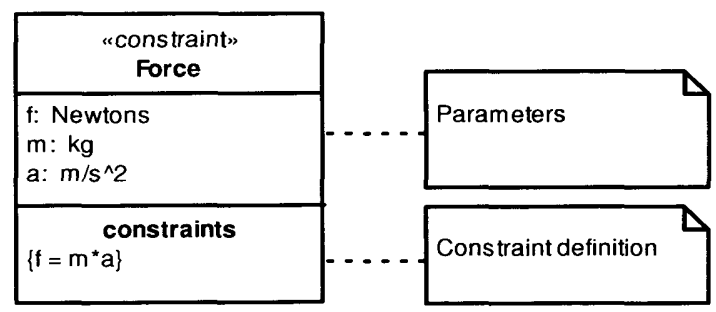

Figure B-22 - Parametrics - Definition Notation

A constraint block is defined using a block (essentially the same as a UML class) stereotyped "constraint" and given a name by which the constraint can be identified. The constraint block has two compartments - the constraints and parameters compartment.

The constraints compartment contains an equation, expression or rule that relates together the parameters given in the parameters compartment. The example above defines a constraint called 'Force' that relates the three 
parameters ' $f$ ', ' $m$ ' and ' $a$ ' given in the parameters compartment by the equation ' $f=m \times a^{\prime}$ ', as shown in the constraints compartment. Such constraints are defined on a SysML block definition diagram (essentially a UML class diagram).

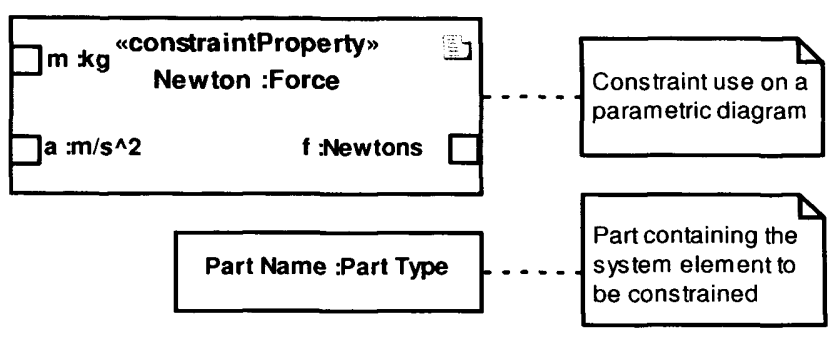

Figure B-23 - Parametrics - Usage Notation

Constraint blocks are used on a parametric diagram using the notation shown above. The small squares attached to the inside edge of the constraint represent each parameter and provide connection points when linking constraints to parts or other constraints.

When used on a parametric diagram a constraint block is referred to as a constraint property and each constraint property should be named thus name :Constraint name. This allows multiple copies of a constraint to be used on a diagram. 


\section{The Escapology Problem}

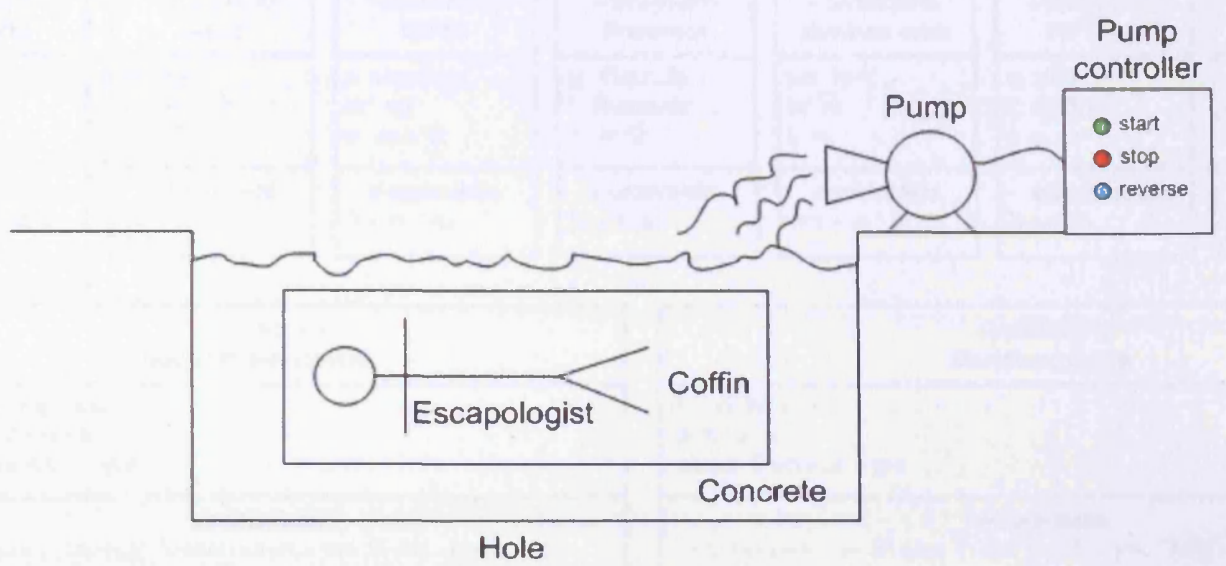

Figure B-24 - The coffin escape

This is a classic escapology stunt that has been performed by many people. It is also a dangerous one, and escapologists have lost their lives performing it because the constraints were not properly understood or evaluated. One such performer was Joe Burrus who died 30th October 1990 when the weight of the concrete crushed the coffin he was in. 


\section{The Definitions of the Constraints}

\begin{tabular}{|c|c|c|c|}
\hline $\begin{array}{l}\text { "Constraint" } \\
\text { Volume }\end{array}$ & $\begin{array}{l}\text { "Constraint" } \\
\text { Mass }\end{array}$ & $\begin{array}{l}\text { "Constraint" } \\
\text { Force }\end{array}$ & «con \\
\hline $\begin{array}{l}v: m^{\wedge} 3 \\
w: m \\
\text { l: } m \\
h: m\end{array}$ & $\begin{array}{l}\mathrm{m}: \mathrm{kg} \\
\mathrm{d}: \mathrm{kg} / \mathrm{m}^{\wedge} 3 \\
v: \mathrm{m}^{\wedge} 3\end{array}$ & $\begin{array}{l}\mathrm{f}: \text { Newtons } \\
\mathrm{m}: \mathrm{kg} \\
\mathrm{a}: \mathrm{m} / \mathrm{s}^{\wedge} 2\end{array}$ & $\begin{array}{l}p: \text { Pas } \\
f: \text { Newt } \\
a: m^{\wedge} 2\end{array}$ \\
\hline $\begin{array}{l}\text { constraints } \\
\left\{v=w^{*} l^{*} h\right\}\end{array}$ & $\begin{array}{l}\text { constraints } \\
\left\{m=d^{*} v\right\}\end{array}$ & $\begin{array}{l}\text { constraints } \\
\left\{f=m^{*} a\right\}\end{array}$ & $\begin{array}{l}\text { cons } \\
\{p=f /\end{array}$ \\
\hline \multicolumn{4}{|c|}{$\begin{array}{l}\text { "constraint" } \\
\text { Decision-equipment }\end{array}$} \\
\hline \multicolumn{4}{|c|}{$\begin{array}{l}\text { pressure: Pascals } \\
\text { strength: Pascals } \\
\text { result: Decision Type }\end{array}$} \\
\hline \multicolumn{4}{|c|}{$\begin{array}{l}\text { constraints } \\
\{I F \text { pressure }<\text { strength } \text { THEN result }=\text { yes } E L S E \text { result }=\text { no }\}\end{array}$} \\
\hline
\end{tabular}

\begin{tabular}{|c|c|c|}
\hline $\begin{array}{l}\text { "constraint" } \\
\text { Surface area }\end{array}$ & $\begin{array}{l}\text { "constraint" } \\
\text { Fill Time }\end{array}$ & $\begin{array}{c}\text { «constraint" } \\
\text { Minus }\end{array}$ \\
\hline $\begin{array}{l}\text { sa: } m^{\wedge} 2 \\
w: m \\
\mathrm{l}: \mathrm{m}\end{array}$ & $\begin{array}{l}v: m^{\wedge} 3 \\
r: m^{\wedge} 3 / s \\
t: s\end{array}$ & $\begin{array}{l}r: \text { float } \\
\text { a: float } \\
\text { b: float }\end{array}$ \\
\hline $\begin{array}{l}\text { constraints } \\
\left\{s a=w^{*} \mid\right\}\end{array}$ & $\begin{array}{l}\text { constraints } \\
\{t=v / r\}\end{array}$ & $\begin{array}{l}\text { constraints } \\
\{r=a-b\}\end{array}$ \\
\hline
\end{tabular}

\begin{tabular}{|l|}
\hline \multicolumn{1}{|c|}{$\begin{array}{l}\text { "constraint" } \\
\text { Decision-stunt }\end{array}$} \\
\hline $\begin{array}{l}\text { breath result: Decision Type } \\
\text { equipment result: Decision Type } \\
\text { result: Decision Type }\end{array}$ \\
\hline$\{$ IF breath result = yes AND equipment result = yes THEN result = yes ELSE result $=$ no $\}$ \\
\hline
\end{tabular}

Figure B-25 - The coffin escape - parametric definitions

\section{The Use of the Constraints}

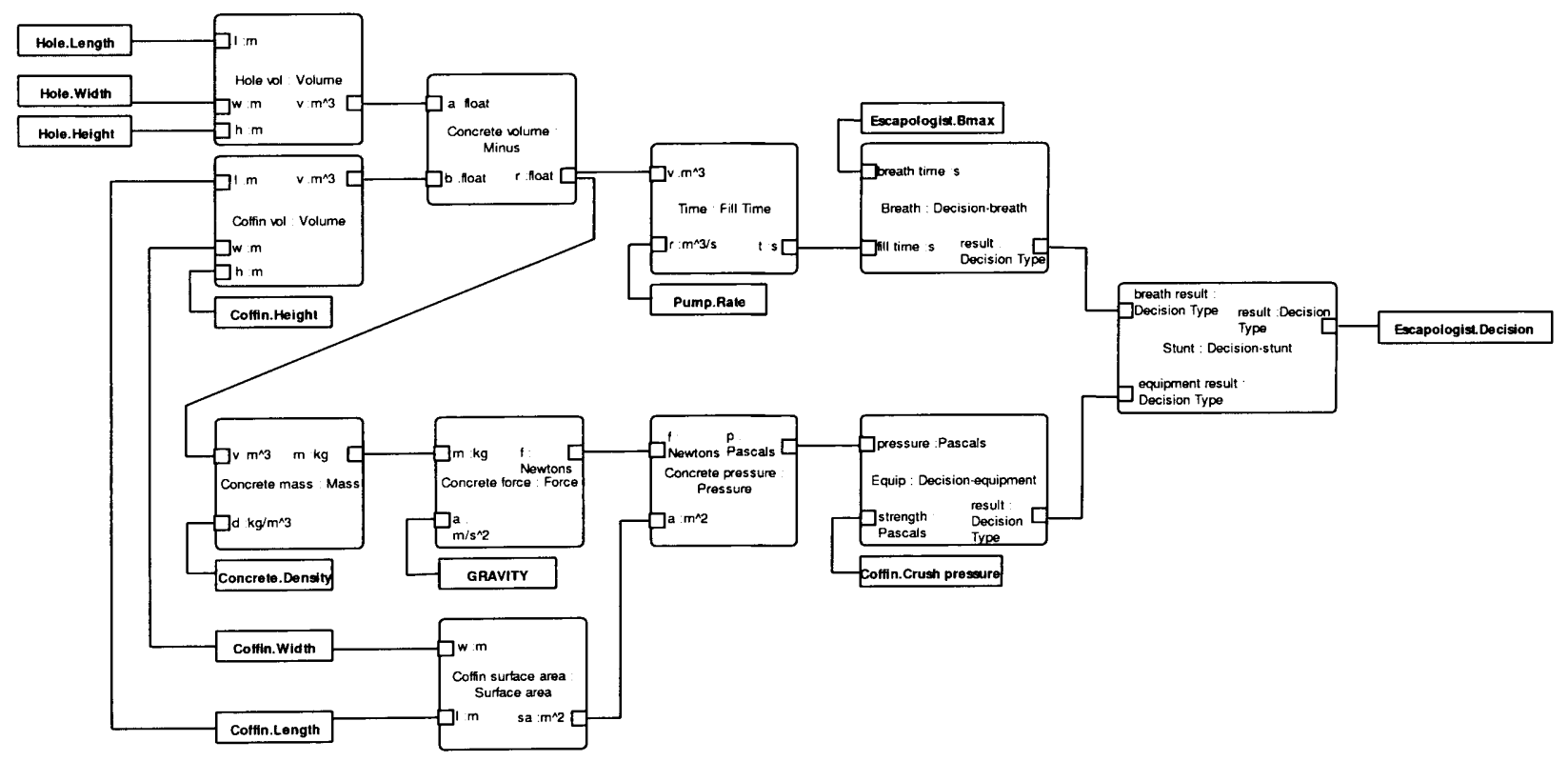

Figure B-26 - The coffin escape - parametric usage 
The sizes of the hole and the coffin are calculated and used to determine the amount of concrete needed to fill the hole. This volume and the pumping rate of the pump are used to determine how long it will take to fill the hole. This forms an input to a usage of the 'Decision-breath' heuristic constraint, along with the length of time that the escapologist can hold his breath, which returns a 'yes/no' decision indicating whether the escapologist can hold his breath long enough.

The volume of concrete needed is used, along with a constant defining the acceleration due to gravity, to calculate the amount of force exerted by the concrete. This force is converted to an exerted pressure using the surface area of the coffin, with the pressure then being compared against the coffin crush pressure in a usage of the 'Decision-equipment' heuristic constraint to return a 'yes/no' result that indicates whether or not the coffin is safe to use.

Finally, the outcomes of these two decisions are used in a usage of the 'Decision-stunt' heuristic to decide whether the stunt should be performed, setting the 'Decision' property of the 'Escapologist' block. In this way the parametric constraints are used not only to specify constraints on the system but also to allow system requirements to be validated. Indeed it may be possible, if parametric constraints can be developed at an early stage of a project, to use them to establish whether a project is even possible long before detailed and costly development work has been undertaken.

It should be noted that this is only one possible parametric usage diagram and is open to significant improvement. However, it gives a good indication of the notation and the way that the constraint diagrams in SysML are used. 


\section{SysML Diagram Relationships}

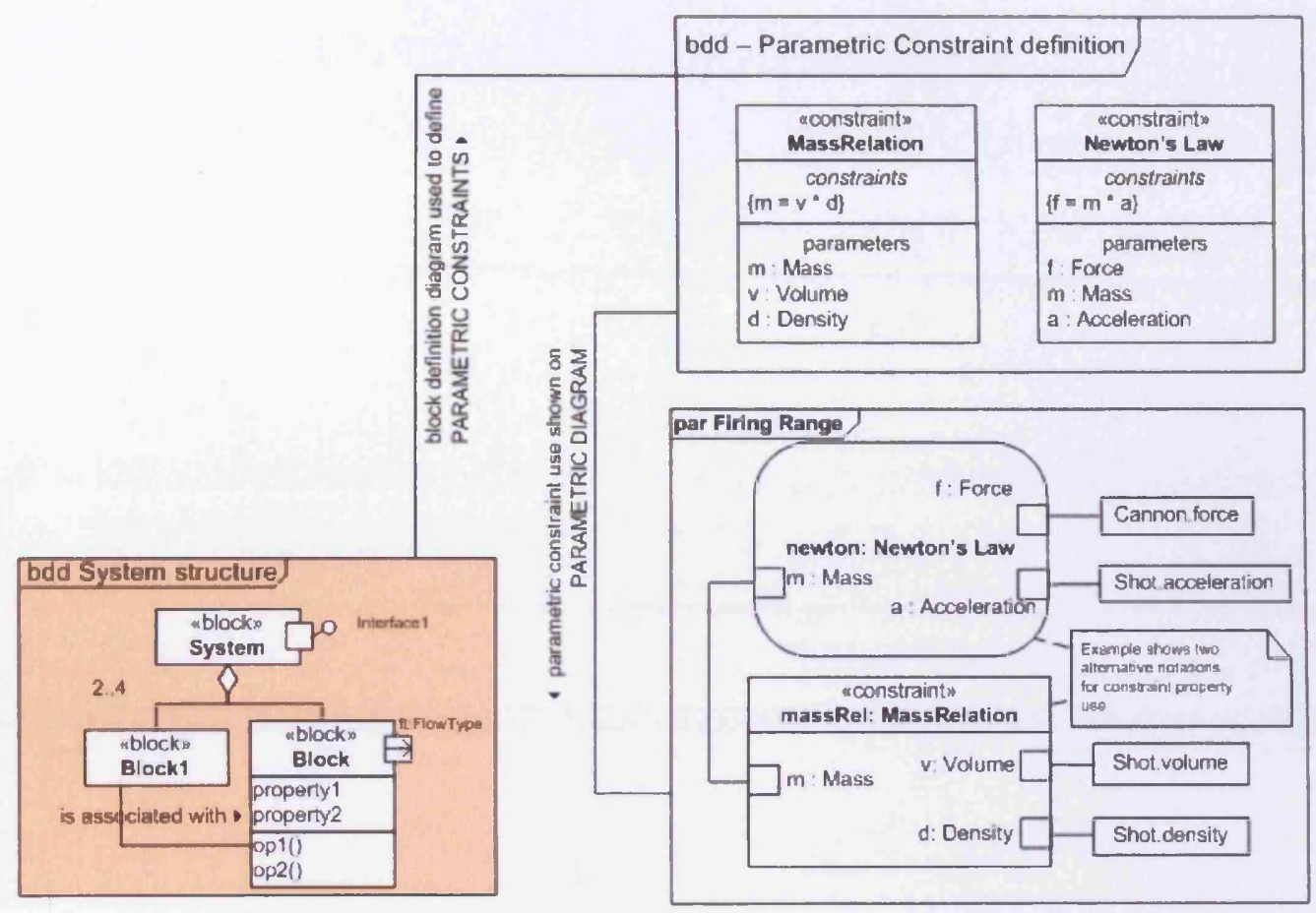

Figure B-27 - SysML Diagram Relationships

Figure B-27 shows the relationships between the SysML diagrams used in this work. It shows the relationship to the SysML block definition diagram which can be considered to be a class diagram for the purposes of this work. The strength of the relationship between the class and block definition diagrams provides a central point from which the SysML profile can be related to the UML. 